UNITED STATES DEPARTMENT OF INTERIOR

GEOLOGICAL SURVEY

\title{
PRINCIPAL FACTS FOR GRAVITY STATIONS IN THE \\ STATE OF CONNECTICUT
}

\author{
compiled by \\ W. A. Bothner and R-L. D. Brace
}

Open-File Report 78-804

1978

$\left.\begin{array}{l}\text { Best copy available. Authors not with USGS \& } \\ \text { no record of where they went--Depositories have } \\ \text { poor copy also. }\end{array}\right]$ 
PRINCIPAL FACTS FOR GRAVITY STATIONS IN THE STATE OF CONNECTICUT COMPILED BY W. A. Bothner and R-L. D. Brace

\section{Introduction}

Gravity data were compiled for the State of Connecticut (fig. 1) as part of an overall effort to update existing DMA (Defense Mapping Agency) gravity files for the purposes of (1) reexamining the gravity field for the northeast United States in terms of seismicity and tectonics, (2) preparing a new series of $2^{0}$ gravity maps to complement U.S. Geological Survey involvement in NURE (National Uranium Resource Evaluation) and CUSMAP (Conterminous United States Mineral Assessment Program) projects, and (3) providing a complete Bouguer gravity map of Connecticut. This report represents the initial part of the latter efforts.

\section{Data Compilation and Reduction}

Data were obtained from the Defense Mapping Agency, DMA Aerospace Center, St. Louis AFS, Missouri 63125 and from U.S. Geological Survey sources. The DMA data represent 59 land-based stations already corrected to the new Potsdam value. The U.S. Geological Survey data (1,505 stations) were collected by R. W. Bromery, M. Davis, and Farrukk Ahmad in 1967; they were tied to a base station at Wesleyan University, Middletown, Connecticut (980305.4 mGal), established by R. W. Bromery from the University of Massachusetts, Amherst (980360.71 mGal) to the Commerce Building, Washington, D.C. (R. W. Bromery, oral communication, 1978). These data were reduced, many from original meter readings, using a digital computer program written by G. I. Evenden and R. R. Wahl and modified by R. Godson, D. A. Dansereau, and R. Sweeney of the U.S. Geological Survey. A correction of -13.7 mGal was applied to the 
Wesleyan base to conform with the 1971 base values of the International Gravity Standardization Net and the DMA data. The Geodetic Reference System, 1967 formula (International Association of Geodesy, 1967), was used to compute the theoretical gravity. The data from both sources were reduced to complete Bouguer anomaly values using a $2.6 \mathrm{~g} / \mathrm{cm}^{3}$ Bouguer density. Terrain corrections were computed from the station to $166.7 \mathrm{~km}$ using a modification of D. Plouff's (1977) program. Curvature corrections were also applied, which yielded total terrain corrections ranging from -0.10 to 6.06 mGals. Tidal corrections were not made because the field data nefessary for this correction were not available. The principal facts for the gravity stations are listed in table 1, which is preceded by an explanation of table headings. 


\section{REFERENCES}

Bromery, R. W., Davis, M., and Ahmad, F., 1972, Simple Bouguer anomaly map of Connecticut: U.S. Geol. Survey Open-File report.

Defense Mapping Agency Aerospace Center, 1974, World Relative Gravity Reference Network, North America, part 2 (?): DMAAC Ref. Pub. No. 25 , with supplement dating gravity values to the International Gravity Standardization Net 1971, 1635 p.

International Association of Geodesy, 1967, Geodetic Reference System, 1967: International Assoc. Geodesy Spec. Pub. no. 3, 74 p. Plouff, D., 1977, Preliminary documentation for a FORTRAN program to compute gravity terrain corrections based on topography digitized on a geographic grid: U.S. Geol. Survey Open-File report 77-534, $45 \mathrm{p}$. 


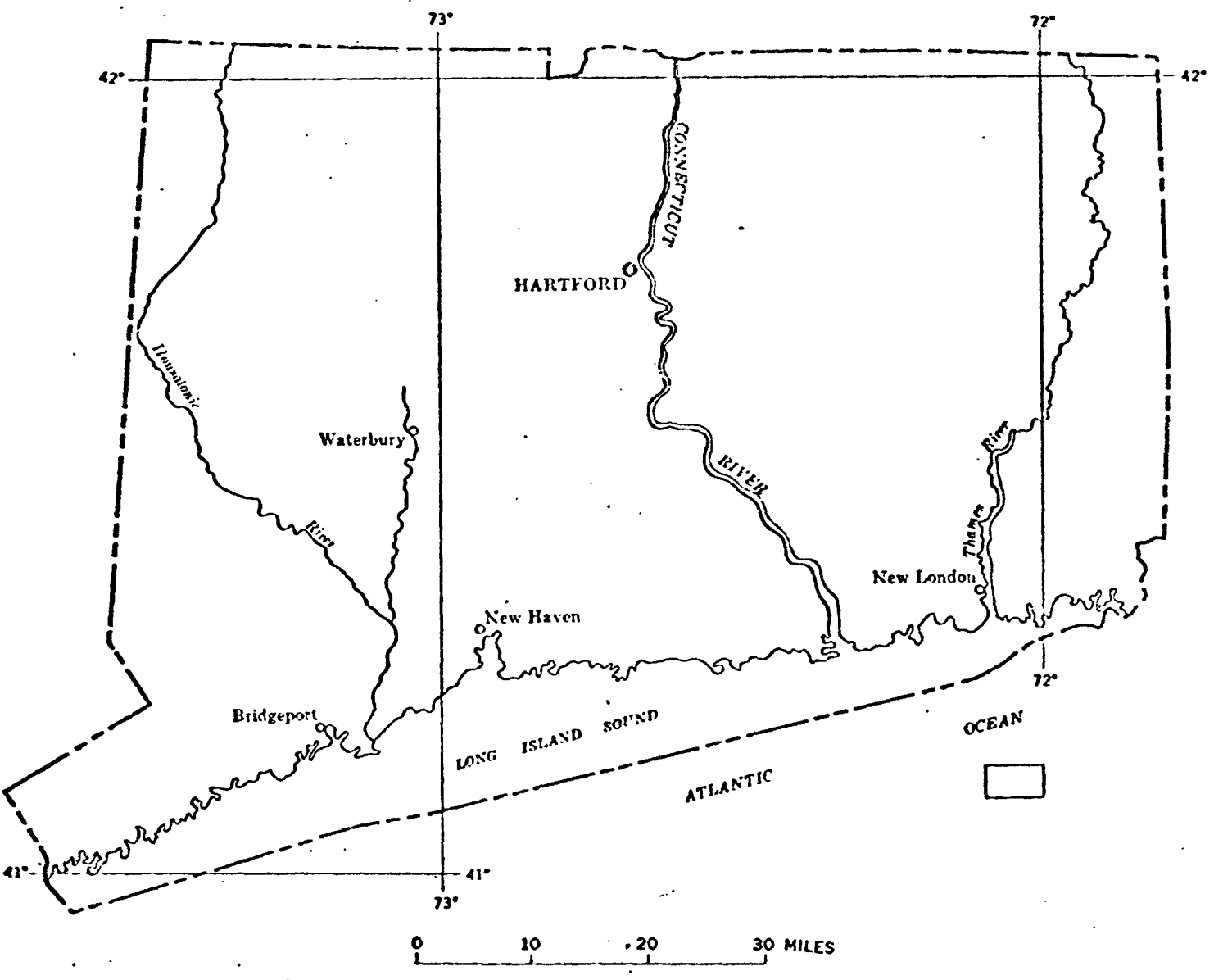

Figure 1 --INIEX NaP OP CONNECTICUT 


\section{Explanation of Table Headings}

STATION IDENTIFICATION

LATITUDE

LONGITUDE

ELEV

ST

OBSERVED GRAVITY

THEORETICAL GRAVITY

TERRAIN CORRECTIONS

FREE-AIR CORRECTIONS

BOUGUER CORRECTIONS

BOUGUER ANOMALY
Gravity station number.

North latitude in degrees, minutes, and hundreds of minutes.

West longitude in degrees, minutes, and hundreds of minutes.

Elevation in meters.

State of station (e.g. Arizona).

Observed gravity in milligals.

Theoretical gravity.

Terrain correction in milligals.

Free-air anomaly in milligals.

Elevation and curvature correction in milligals.

Bouguer anomaly in milligals for assumed average density of 2.67 $\mathrm{g} / \mathrm{cm}^{3}$. 


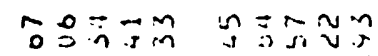

mi $\dot{0} \dot{\pi}$

$00 \tilde{m}=$

$\therefore \dot{0} \dot{0} \dot{0}$

กำ

กิำ:

$\approx \approx m n \Omega$

光宁范

$\because \sim N=$ $\therefore \dot{0} \dot{0}$

$\dot{0} \dot{\sim} \dot{\sim} \dot{\mathrm{i}} \dot{\mathrm{i}}$

a $u n j$ in

inmmi

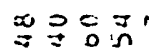

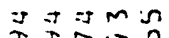

证学要至

0.5000

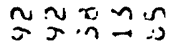

mongs

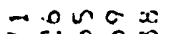
$\therefore \dot{x} \therefore$ $\dot{0} \dot{0} \dot{i} \dot{0}$

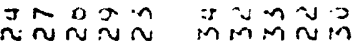

minmin

min in:

inja

$r-50 \approx=0$

$30 ? 30 ?$

93338

23330

32009

ல்

¿ंव்:
잉? $\dot{0} \dot{0} \dot{0} \dot{0}$ $\because \because \pi$ บ

พ v $\sim \sim$

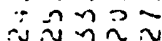

ก.บ?

$\dot{j} \dot{j} \dot{j} \dot{0}$

$\Rightarrow$ in $\begin{aligned} & 0 \\ & \text { i }\end{aligned}$

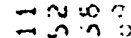

in is

nกำ

$\dot{0} \dot{0} \dot{0}$

$m \stackrel{\infty}{m}=$

$0=0 ?$

○ं०்:

inni-

$\therefore \therefore 0 \div$

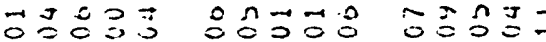

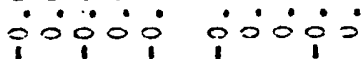

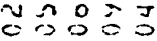

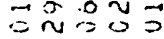

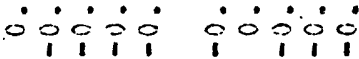

○ं0ல்

$\therefore 0 \dot{1}: \dot{0} 00 \dot{0} 0$

อกำกร

in

$\tilde{\sim}=\vec{\sim} \bumpeq \hat{\sim}$

$\overrightarrow{0} \ddot{\sim} \ddot{\sim} \stackrel{0}{*}$

\section{$0: 0<0: 0$}

$0 \rightarrow \infty n: 0$

$\because: 0 ;:$

$\because \dot{1}_{1}=\dot{1}$

نं iि: OTin

$\because \because ? \because$

1

$\therefore \quad 2500$ $\because m \dot{0} \div \dot{0}$

$x \geqslant 2 x ?$

$m n o \approx m$

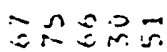

$\dot{0} \dot{0} \dot{0}=\dot{-}$

$v \approx D \cdot m \vec{J}$

$\dot{\sim} \therefore \dot{0} \dot{0} \dot{0}$

$\dot{c}=\dot{0} \dot{0}$

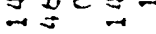

$\dot{\sim} \sim \dot{\sim} \sim \dot{n}$

กิ่งกิน

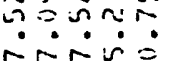

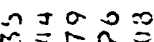

í⿺辶்

$\therefore \dot{0} \div \dot{0} \div$

$\because \overrightarrow{0}:=$
$\because \div \frac{1}{1}=$

?

$\therefore \dot{0} \therefore$

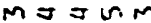

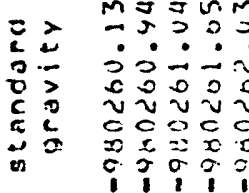

คิำตับ

$0 \pi \ln -\infty$

$000 \Omega 0$

- . . .

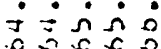

$v \sim \sim v$

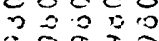

vิs

$v v v v$

$0=000$

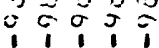

$\therefore 0 \dot{0}=0$

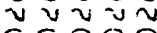

00300

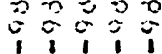

$\hat{\Omega} \backsim=\hat{\Omega}$ $\because \dot{0} \dot{0} \dot{0}$

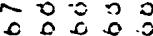
v v v v v

$x$ : 000

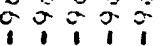

จง

Non oro

우음

$\sim \sim \sim \sim m$

ก ก

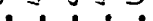

- - ja

กับ

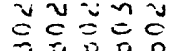

$200 \pi=$

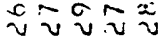

$c=000$

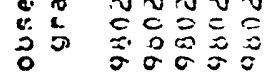

o 000

ก.0 0

$\dot{j}=\dot{i} \dot{0}$

ป.

$\because \approx 00 \div$

ก

$\dot{0} \dot{0}=\dot{0}$

x̃v心

$\begin{array}{llll}0 & 0 & 0 & 0\end{array}$

amNi

$\therefore \dot{0} \dot{0} \dot{0}$

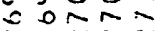

v v v v

$\Rightarrow 0=$

\%

$=-\infty \sim 0$

$n-z=v$

jum $\dot{0}-\dot{0}$

ก

$\begin{array}{llll}0 & 0 & 0 & 0 \\ 0 & 0 & 0 & 0\end{array}$

. $000 \sigma$

ณ บㄴํㄴ

ธேธ兀

๒こピே

눈

ேㄴㄷㅜ

$\div 5-5=$

mกองท

E 윰ำ

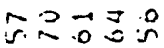

กิำ

กำณ

$\therefore \therefore \sigma c i$

$\ddot{r} \dot{v}=\dot{\sim} \dot{v}$

$v=\sim \dot{0} \dot{0}$

$\ddot{\sigma} \underset{\sim}{\sim} \dot{0}=$

กั

Mำ $\cong$

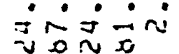

$=0 r . m m$

$\rightarrow v \in C$

$\ddot{1} \ddot{2}=\overrightarrow{0}$

¿vvv

$=000$

\%

$0 \sim \Rightarrow D M$

-?วงก

วงนส

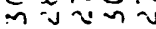

$c=j c 0$

i) 0 D

政

$-\frac{2}{x} \simeq$

$\dot{v} \dot{v} \dot{n}$

№n

$\begin{array}{ll}v & 0 \\ = & 0\end{array}$

ज्ञ

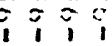

$=\sim \sin$

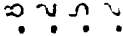

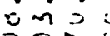

ทn一

co을

向。家

可它

万人:

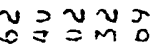

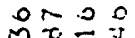

ヘำi

$\int_{0}=$

0 을

$\because 2$.

c

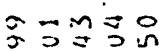

5200

$\infty \rightarrow 00: 4$

in $0 \div$

บทำ

$\cong x \in \operatorname{cs} \approx$

$000 \mathrm{~cm}$

$\operatorname{oin} \sin c \cos$

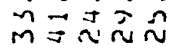
$\dot{0} \dot{0} \dot{0}$ jiñ

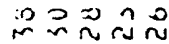
$m=N n n$ $n m m n$ نेंì $\sin \frac{50}{4}$ $\dot{1} \dot{\sim} \dot{0} \dot{0}$

$\exists \approx n n$

วของ vin

$m m \sim m m$ mMmMm ninmm

$\hat{1} \div \hat{1} \hat{1}$

$m \cdots n \div \frac{1}{1}$

$m m m m n$

$m m n m m$

$m m m n$

Ti:i

$n m m m$

는

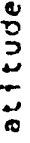

magno

$\cong n$ 只

m人무

MN $\sim \infty \sigma$

$\overrightarrow{4}$ 으의

o-man

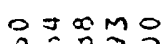

जisi:

$\dot{0}: \dot{0}:$

$\therefore \therefore \dot{0}: \dot{0}$

வ் $\dot{0} \dot{0} \dot{0}$

$\ddot{\vec{g}} \overrightarrow{-\vec{J}} \vec{\Xi}$

$\vec{I}=\vec{J} \bar{J}$

$\vec{\Xi} \breve{\Xi}$

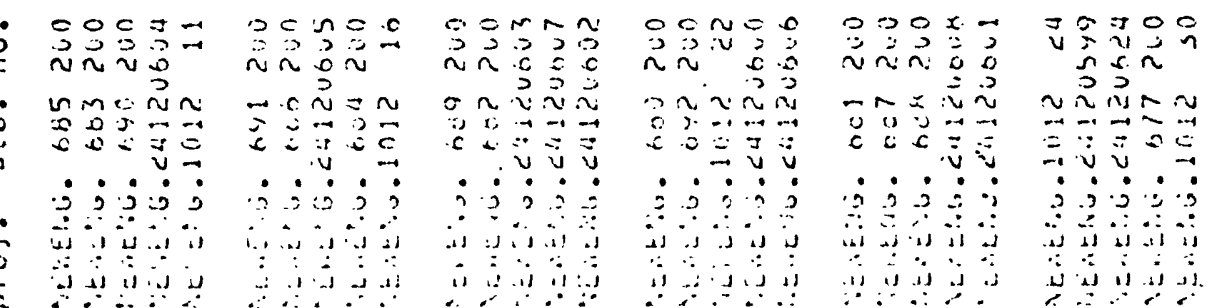

$\vec{\Xi} \vec{\jmath} \vec{\exists}$ $\overrightarrow{. J} \vec{\exists} \vec{J}$

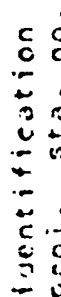

$2+\frac{1}{x}$

$>0$

a

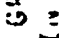

焉。

这这

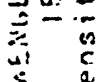

$\stackrel{0}{2}$ 
ox $x=$

ำ

ㅋNㅡำ

ज酧完

$\stackrel{0}{*} 0$

E

E

:

:o.

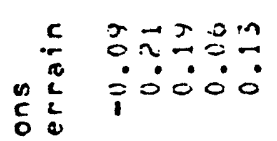

20J.
$0000 \div$
$0 \div 000$

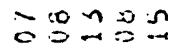

$\therefore=0 \dot{0}$

$n=0 ; 0$

Dinoso 0

uั

2.

i c

$\therefore \therefore \therefore \div$

RAMCM

$\pi \geq 039$

$\therefore \dot{0} \dot{i} \dot{r}$ i $\dot{0} \dot{0} \dot{0}$

111

g $\approx \approx \operatorname{mon}$

$\dot{\therefore} \therefore \ddot{\sim} \dot{\sim} \dot{\sim}$

i
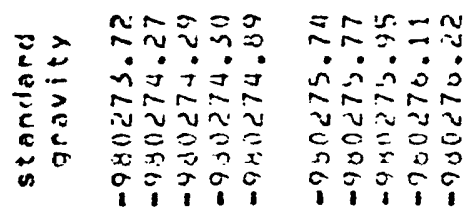

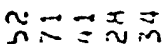

กㄴ.? $\div$

$20-\infty=0$

$v v v v v$

00000

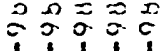

mnn

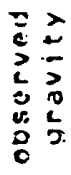

$\begin{array}{ll}2 & 2 \\ 3 & 2 \\ 2 & 2 \\ 0 & 0 \\ 0 & 2 \\ 0 & 0\end{array}$

o

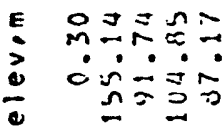

\section{$\dot{=} \dot{\dot{j}} \dot{\sim}$ \\ c

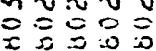

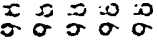

$\approx$ 융키

$\dot{0} \dot{\sim} \dot{n} \dot{0}$

는은

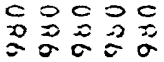

momon

$\vec{\nabla} \dot{0} \cdots$

$x \geq x^{\prime}=$

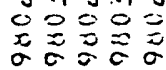

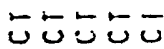

ว $2 n>0$

$r=0 \overrightarrow{0}$

- $\dot{0} \dot{0}$

$\begin{array}{ll}c & 9 \\ c & 5 \\ 0 & 0 \\ 5 & 0 \\ 0 & 0 \\ 0 & 0\end{array}$

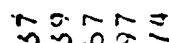

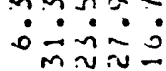

$m m m \backsim m$

$\because \div \div \frac{1}{1}$

옹ำ

$\dot{\vec{v}} \dot{\dot{u}} \dot{a}$

mimmm

$\pi \frac{1}{1}$

อำ.

$\pm 0 \dot{0}=\vec{I}$

$m:=N-$

no ำ

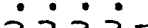

$202 \geq 2$

ज可可

$\because \ddot{0}=\dot{v}$

의의 의

vivin

$\vec{\nabla} \vec{\nabla} \vec{ज}$

ज可

MCN 0

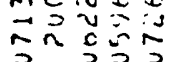

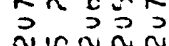

$\underset{n}{\sim} \simeq \simeq$

v $c \vec{i} \vec{u}$

$\dot{0}: \dot{0} ;$

$5.9=0.9$

c)

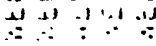

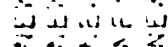

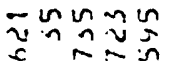

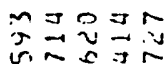

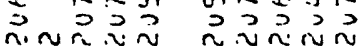

-

$\Xi \vec{c} \vec{j} \vec{j} \vec{J}$

$\vec{\exists}=\vec{\nabla}$

$\therefore \therefore \therefore: \dot{2}$

in 4 it is

? ? ? ? ?

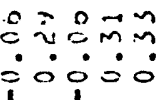

000
000
00000
000

$\because \because=00$

: $\dot{0} \dot{0} 0$

$\hat{0} \tilde{0} \cong \tilde{n}$
$\therefore \dot{0} \dot{0} 0$

वृ:

$1 \%$

11

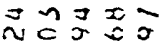

ำำ

ํำำำำ

원

inis

$\because=\dot{\sim} \dot{0}$

$\eta 0 J x: n$

$\therefore \dot{0} \div 0$

$\div 0 \%$

ว으을

그응응

ำ

$=\dot{0} \dot{0} \dot{m}$

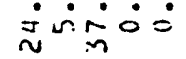

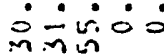

ga

$\therefore \therefore \dot{0} \dot{m}$

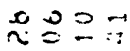
$\dot{0} \dot{0} \dot{ }$

$\approx 5=0$ in

ง? 0

$D 2 x=5$

v vบ-

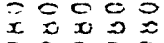

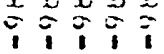

นับ

ำกำก จㅇำ ข้นีข

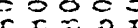

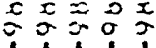

$\cong=00$

$\therefore \dot{-1}=\dot{\Xi}$

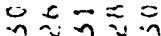

$\begin{array}{lll}0 & 0 \\ 0 & 0 & 0 \\ 0 & 0 & 0 \\ 0 & 0 & 0\end{array}$

융ำก

vinni-

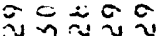

$=0000$

กกก

23007

v v $v$

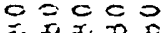

c $=0$

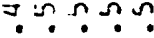

$\vec{x} \vec{x} \vec{x} \vec{x}$

viv $v$

co 000

주워

$\rightarrow 20$

$\dot{1} \dot{0}=$

11

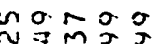

$\operatorname{cin} 00.0$

이웅

ㄴำ

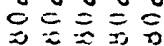

$\dot{0} \dot{0} \dot{0} \dot{0}$

x $\sigma v \sigma v$

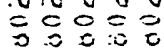

$$
47
$$

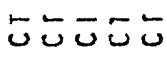

ேーーேட

$5--$

눈두

$\eta 0=N ?$

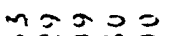

nono:

$\because \because \cdots$

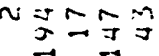

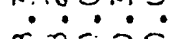

$x x=00$

$\therefore \dot{0} \div \dot{0}$

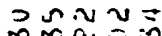

m.

$\therefore \dot{m}-\dot{0}$

Sing

$\vec{x}=\mathbf{v}$

v

$\Rightarrow 05$

a 50

لّ

บ? ? ?

n.vNำ

v v.v u.u

둥ำ $\therefore$. 1 14

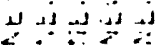

ำ $\therefore \dot{\sim} \dot{0}$

nonNm

กอก $\dot{0} \dot{\sim} \dot{v}$ in

in mmm $\div \div \div \%$

MMMNN $\div 11 \%$ กำ? กำ

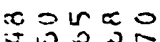
$\div \div \div$ ?

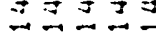

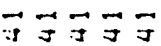

$\because \vec{J} \vec{J}$

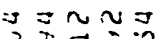
요에 ur ùv $\simeq \square \simeq \simeq$ $\vec{\nabla} \vec{\nabla} \vec{j}$ ป v v d $j ; j ; 5$

ل

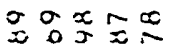
$\dot{v} \dot{i} \dot{\sim} \dot{j}$ Nññ $m m M N N$ $1 \% \div \div$

$m \equiv \pi \cdot n$

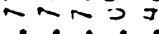
J $\bar{\Omega} \Omega$

于 $\vec{\Xi} \vec{\Xi}$

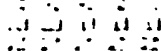

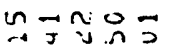
$\because 35$ ก UกN $\vec{\exists}=\vec{\Xi} \vec{\exists} \vec{\nabla}$ u v v v ; $; 2$ נ4 $\because$ w

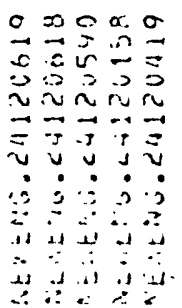

m况 $\vec{v}$ $\therefore \dot{0} \dot{0} \dot{0}$ m约心 NNNMm $\div \div \div \div$

c. in in $x$ $\Omega \Omega \Omega \cdot$ กंin

च

s: $=0$ $\dot{j} \dot{0} \dot{\mathbf{O}}$

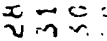
이일 ब云宗

ธ5ธ

ब可可 inj:

구․ ñن NMN 1.11

$\sin n$ $\therefore=5$ กรก

$\vec{\exists} \bar{J}$

or un a $x$

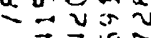


0
5
0
0

c)

$\vec{n} \cong=0$

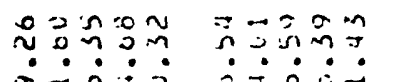

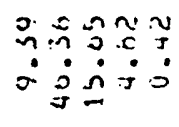

30339

ல0:0்

o-n n

$\begin{array}{lll}0 & 0 M 0 \\ 0 & 0 & 0\end{array}$

ct

บै

- $00=0=1$

- ?

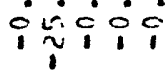

$\pi=\pi 2 \pi$

$\because \because \because \div \div$

$\therefore$

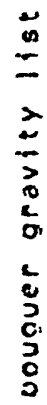

is

2

$\vec{\omega}$

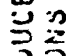

แ⿺辶一兀

$\approx \frac{1}{x}$

$\geqslant 0$

范

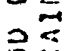

$\approx$ 空

1.

范 $\begin{array}{ll}3 & 2 \\ 2 & 0 \\ 3 & 0 \\ 0 & > \\ c & 0 \\ a & 2 \\ 0 & 2\end{array}$

$m m 0=0$

$\dot{v} \dot{v}-\dot{j} \dot{v}$

viv $v$ v

coc 00

\%

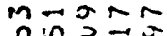

$\begin{array}{ll}3 & 2 \\ 3 & 0 \\ 2 & 3 \\ 0 & 3 \\ -0 & 2 \\ 0 & 5\end{array}$

ڤั

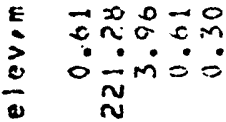

$\vec{c} 0 \dot{0}=$

- $0=$

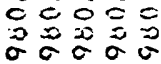

- $-5=$

$\rightarrow \infty 0-0$

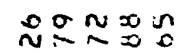

ㄴำ

$\div=$

岂

in $\dot{\sim} \dot{\sim} \dot{\sim} \dot{0}$

NN.UN

$\because \div \div \frac{1}{1}$

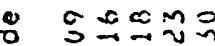

$\because \div \div ท$

$\dot{0} 0 \dot{0} 0$

$\vec{a} \vec{\Xi} \overline{\vec{g}}$

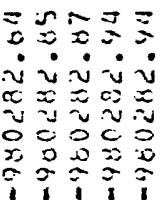

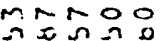

$\dot{0} \dot{0} \dot{0}$

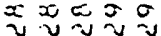

$\begin{array}{lll}0 & 0 \\ 0 & 0 & 0 \\ 0 & 0 & 0 \\ 0 & 0\end{array}$

பேேே

$\Rightarrow 3=N$

ヘै: $\dot{0}=\dot{0}$

$\stackrel{n}{2}$

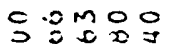
$\dot{i} \dot{i} \dot{i} \dot{0}$ लũmm

m Nnn

$\div \div 11$

D

:

$\therefore \dot{0} \dot{0}$

ज可宁可

กั0

<宁

织记旅

$\dot{0 j} \dot{j} ;$

$\therefore \quad 302$

:

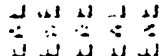

告

$m \equiv N \cong \Omega$

$\therefore 5$

ñuna

$\because \vec{\nabla} \vec{J} \vec{G}$

บง บับ

? ? ? ?

4

$\therefore$ ذ $\overrightarrow{\vec{v}} \overrightarrow{\vec{v}} \overrightarrow{\vec{v}} \overrightarrow{\mathrm{v}} \overrightarrow{\mathrm{v}}$

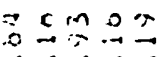

$\dot{\sim} \dot{0}=\dot{v}$

$=\pi \frac{1}{2} \approx 5$

$\ddot{i} \dot{i} \hat{i}$

$\approx \approx ⿻ 0$

$\therefore \dot{i} \dot{0} \dot{0}$

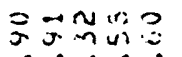

ㅁํㅇํำ

$\dot{0} \dot{0} \dot{0} \dot{0}$

$\dot{0} \dot{\sim} \dot{\mathrm{v}}$

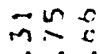

$\therefore \dot{0}$

$n \geq n^{1} n=$

c⿻一𠃋ำ

$\dot{i}=\dot{1}=0$

ำกำ

$\dot{2} \dot{2} \dot{m}$

$\dot{n} \dot{0} \dot{0} \dot{0}$

J马ำ

¿i:

등ํำ

$\dot{0} \dot{i}$

en $n$

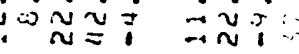

03230

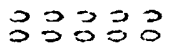

20303

영영

응워웡

뭉웅

ல்0ல்

ப்0ல்

옹응

0ல்

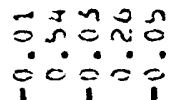

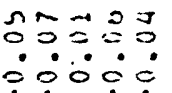

om.un 0

กอบำ

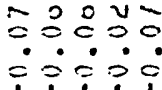

$1 \%: 00$

1 i i

$\therefore 0 \Omega=n$

$\because 2 m 0 \%$

iिĩi c:

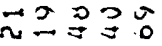

ติดกำ

$\ddot{i} \dot{i}=\dot{0}$

ioini

$5 x=52$

ลำำ

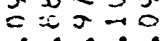

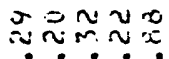

$\dot{m} \dot{m} \dot{0} \dot{0}$

のっコ一

$\approx 00 \approx \pi$

$c+-10$

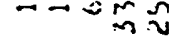

$r=\pi \sim r$

$\because \ddot{\sim} \dot{n} \dot{m}$

$\sin x=\infty$

이을

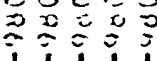

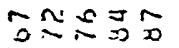

$\hat{\omega} \sim \approx \sim \sim n$

v v v

$\Rightarrow 00000$

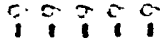

minco

$\therefore \dot{j} \dot{i} \dot{j}$

$0 \approx c^{x}=$

$\begin{array}{lll}0 & 0 \\ 0 & 0 & 0 \\ 0 & 0 & 0 \\ 0 & 0 & 0\end{array}$

$\simeq 0 \approx 05$

$\dot{v} \dot{v} \dot{=} \dot{v}=$

v

ขบข

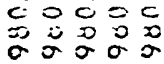

r $m s$

Dว $ว 5 \div$ $\ddot{x} \dot{0}=\dot{0}$

uv v

0000

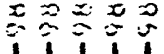

กำ

$\dot{0} \dot{\vec{z}} \dot{0}$

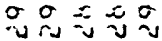

$\begin{array}{lll}0 & 0 \\ 0 & 0 & 0 \\ 0 & 0 & 0 \\ 0 & 0 & 0\end{array}$

ேํำ

ーㄴ

スッทั

Dos-o

는

Nin $0 \dot{0}=\dot{0}$

뭉

준은

-

$=\underset{0}{0}=0$

จนสนด

$\because \because \div ?$

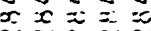

ง v $v$

-

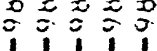

$\% 0=N 5$

$\therefore \dot{0} \div \dot{0}$

$\sigma=0 \approx$

c $0=$

क人

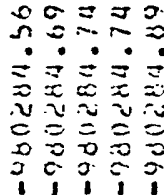

$\Xi \approx M D=$

ن ن

$\exists \vec{\sim} \hat{c} \hat{\sigma}=$

$\therefore \therefore$ i

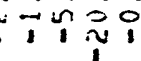

$\because$ 등:

$\dot{0} \therefore$

111

nos:?

$r \div \div=$

-MO

$\cos =0$

$\operatorname{4ñ2} 2$

$\bar{x} \dot{x} \geqslant \vec{x}$

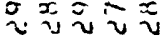

$00=0$

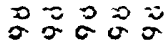

$55 \div 55$

$5-5-5$

$00 \Omega 0 m$

$\vec{n} 亏 \vec{c} \ddot{\sim} \vec{c}$

isisir

$\dot{x} \dot{=} \dot{0} \dot{0}$

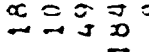

$\because \approx m i n$ m

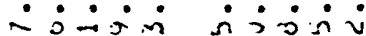
nm $\vec{n}^{\circ}$

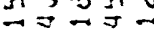

NNUMm

ก N

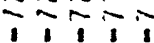

$1 \% 1 \%$

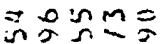

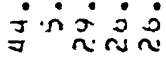

nim:Mn

$\div \div \div \div$

conn

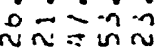

$\sim \sim \sim \sim r$

$\because \div \div \div$

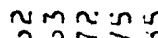

oninon

$n 0 m$ no

i. $\dot{0}: \dot{0}$

$\therefore \div \div \div$

$20=2=$

$\vec{\nabla} \vec{\nabla} \vec{ज}$

可可学宁

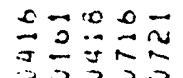

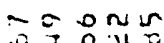

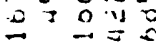

วnก?

$\simeq \simeq \simeq \simeq$

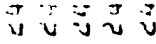

$\therefore \therefore ; j$

is $+1+1$ is

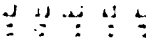

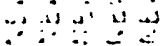

$\because \because ! \quad y$

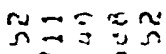

는

$\simeq \underline{-}=$

$\vec{v} \ddot{v} \vec{v}=$

$? \because \vdots ?$

is $\because y$

$\therefore \div$ w $2 \overrightarrow{2} 2 n$

จ 
온 $\rightarrow$

$\dot{i} \dot{x} \dot{0}$

a ONOD

$\therefore \circ \dot{n}=$

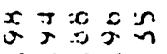

:

is ?

है:

omnin

อุด?

$\Re x=0: 0$

ㅂコㄹ

نं $\dot{0} 0$

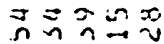

$\ddot{\sim} \dot{\sim} \dot{0}$

一心

33003

.

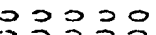

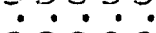

영으의응

○ं0ல0

$0035 ?$

$\therefore \dot{0} \dot{0} 0$

ว 3 ㄱํำว

00000

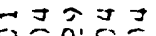

$\hat{0} \overrightarrow{0} 00$

車

$\therefore 0^{\circ} \circ 0^{\circ}$

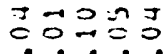

نं०्:

โดํํㅇํㅇ

i०0ं

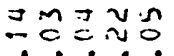

○ंi்:

,

造

ᄂ

ㅇํㅇ

çoñ

ị:

츤ำ

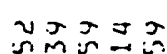

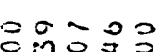

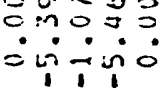

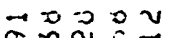

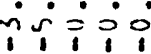

$0 x \geq 2 y$

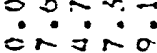

$\therefore=50$

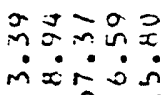

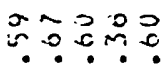

वं்

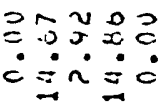

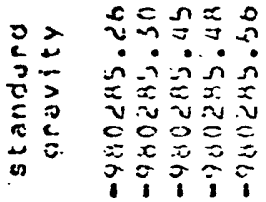

$=0$ ก

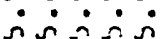

$x=x=$

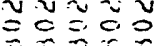

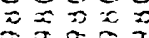

जि

in전 $\vec{n}=$

3

2:

:

- $\cdot$.

웅

cट्ㅇำ

omscun

$\because x \pi$

$\dot{x}=-\dot{0}=$

讨记

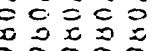

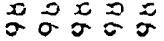

\&

ษังヒこ

テこここす

E mpiñ

过它完

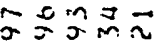

$\dot{0} \dot{\Delta} \dot{\bar{s}}$

$0=0 m a$

$\dot{0} \dot{0} \dot{0}$

$x x \approx x \approx$

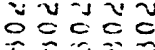

11

mana

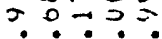

$\dot{x}-\dot{0} \dot{0}$

vivi

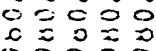

U大。

こテษั

ㅇํ욤ำ

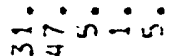

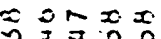
$\dot{0} \dot{0} \dot{0}$

บ $\tilde{v} \tilde{0}$

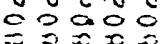

5
$1 \%$

우의

$\because \div 30$

$\dot{s} \dot{v} \dot{0}$

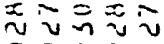

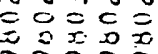

のるする

ヒேこここ

马ी ใํํ응

$\therefore \dot{0}$

in $0 \overrightarrow{0}=$

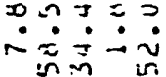

ภु

nnNing

ㄴำ

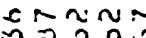

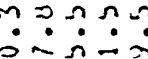

on inom

$? \because \simeq \equiv$

的实宁

m N

$\sim \sim m \sim \sim$

$\sim \sim N \sim$

$\div \div \div \div$

언ำ

$0 \approx-100$

$\dot{j} \dot{\vec{j}} \dot{\sim} \dot{\sim}$

กNNAN

$\div \div \div \frac{1}{1}$

引े

พิ

هُ

美

2

$=$

ج完:

范

范。

这落

宩出

要

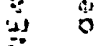

nn $x$ 의

四읏

Nin ing

On

- $0: \dot{0}$

$\dot{0} \dot{0} \dot{0} \dot{0}$

$\dot{0} \dot{0} \dot{0} \dot{0}$

00000

$\dot{0}=\dot{0}$

$v i v i$

$x \equiv \equiv$

;

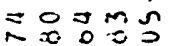

$\dot{i} \dot{i} \dot{v}$

ป v

$\underline{V}=\mathcal{V} v$

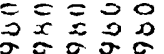

ํำㄴ?

$\vec{\nabla} \vec{\nabla} \overrightarrow{9} \vec{J}$

新㲾

ज可宁

$\therefore \dot{0} \leq \dot{0}$

$\vec{\nabla} \vec{J} \vec{ज}$

-ேㄷ

$=20=2$

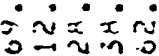

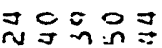

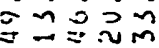

N $\sim M \sim$

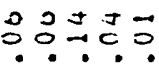

íं

니요요

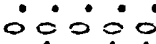

$m=2$

23039

30903

○ंவ்

옹원?

ن 00

이르ำ

ำง

ñ 0 in

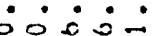

cim-i

$\dot{0} \dot{0} \mathbf{n}$

$2 \div 11$

- $3 x^{2}$

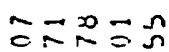

ละว?

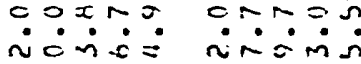

iñ

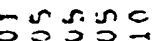

$m \approx n \approx m$

$x \equiv=0$

viviv

이이

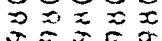

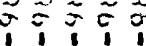

$\neq=$

$-v \tilde{v} v$

$0=000$

के

잉도

บำำ

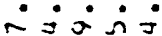

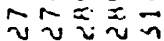

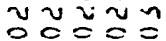

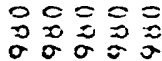

in $\dot{0} \dot{0}$

ㄱำ

00000

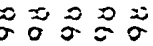

$m=15=$

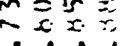

无文

$v y$

oㄷ․

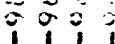

$\stackrel{2}{=} \leqslant i$

$\therefore \dot{0}=$

riv

v

00

ஸேர்

பேர்5

$-5-$

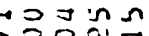

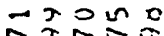

$m=n s$

¿0ंi:

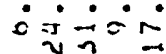

Dic

$\infty \ln n \pi$ n

in 0 - no $\dot{0} \dot{0} \dot{x} \dot{x}$

$70 \div 52$

ำ v $\dot{x} \dot{n} \tilde{n}$

的气

งกmกN 
en

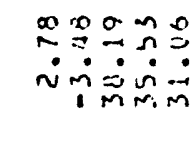

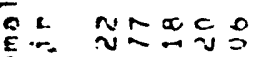

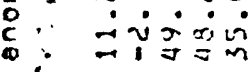

$\div$

-

¿ $0 \dot{0} \dot{0}$ a.

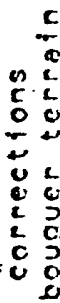

RNA

$00 \div \div 0$

$0<000$

$\vec{c} \overrightarrow{M O C} \overrightarrow{0}$ वosi:

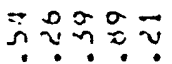

$\dot{J}=\dot{\vec{N}} \dot{x}: \dot{p}$

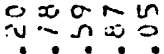

$\dot{v} \dot{v} \dot{i} \dot{0}$

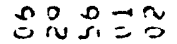

$\dot{-} \dot{\sim} \dot{\sim} \dot{0}$

$\because \pi$

$\approx 0.0 \pi$

กำn

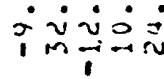

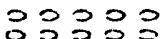

운궁영

$\therefore 0000$

○ंब

옹응

0ं0்:

영요영

○ं0்0

$\therefore \equiv \cong \approx \overline{0}$

$\approx-1 v 0 n$

둥으.

$\dot{i}=0 \circ \dot{0}$

i i:

$v=-v m$

$0=00$ isisi

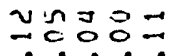
-0000

곤

․ㅜㅇ. ०0:00

ภำะด $\dot{0} \dot{0} \dot{0} \dot{0}$

$m \backsim x x z$

$\Rightarrow \approx n=0$

웜

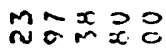

0 요의

$\dot{\sim} \dot{\sim} \dot{n} \dot{\bar{n}} \dot{m} \dot{m}=\dot{0} \dot{=}$

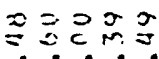

isionis

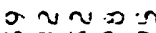

- naro

$\dot{0} \cdot \dot{0}$

भiñis

․ㅜㅇ

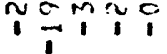

$\sim \overrightarrow{2} 3=\sqrt{2}$

$\therefore \dot{0} \dot{0}$

$\cong \overrightarrow{M ำ ~}$

$\therefore \therefore \dot{0}$

$\Rightarrow 0 \ln x$

$\simeq \equiv$

$\therefore \tilde{a} \leq 0$

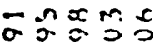

0
02
0
0
0
0
0

$\therefore \therefore \dot{0}$

$x \approx \approx 0$.

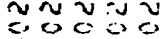

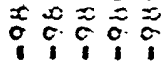

omm

$\because \because \because \because ?$

$x 0=x$

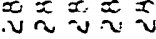

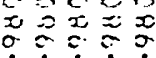

ㄴำำ

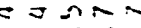

这这这

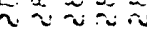

$\because-20$

c०

$O \bar{x}=m$

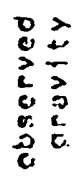

$\dot{0} \dot{0} \dot{0} \dot{0}$

记运

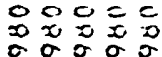

in 0 皮

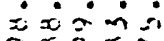

xac

ن v

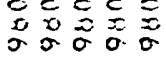

: ヒேこัテ

テこテこテ

윰유융

-

o.unon

$\because-x \geq \approx$

플

ㄴํำ

$\therefore \dot{0} \dot{0}$

is

กิน

o

$n s=\sim n$

$x=00$

$\therefore \dot{0}=\dot{z}$

iv $\approx v$

c

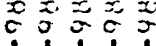

$\tilde{N}=\sim c$

$\sigma 5=\nu m$

$\dot{0} \dot{\sigma} \dot{\sigma} \dot{0}$

vivu

$\sum_{0=0}=0$

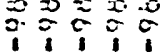

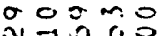

a $\dot{0} \dot{0}$

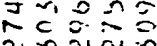

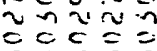

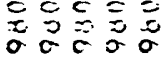

cus vo

v $v=x$

$\therefore \therefore \therefore \div \therefore$

$\sin =\frac{\pi}{\sim}$

원

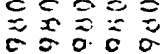

ேำ5

ேํㄴำ

ñano

Une n=

$\tilde{n}=\sqrt{2}=0$

$\infty \sim==$

$\dot{\sigma} \dot{\sim} \dot{\sim} \dot{0}$

๒ேธப்

ron-

$=00 \cong$

$\dot{x}=\dot{m} \dot{0}$

$\vec{\nabla} \vec{m} \vec{m}$

구요

$N=m$ on 20

$0 \div \div=\frac{1}{1}$

$\therefore \div \div \div$

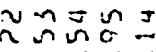

$\therefore: 0 x 0$

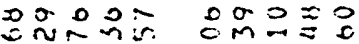

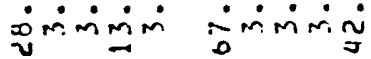

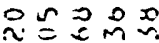

$\dot{\therefore} \dot{\sim} \dot{\sim} \dot{v}=$

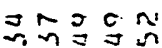
$\dot{a} \div \dot{a}=\dot{2}$

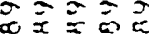

$\operatorname{lin}^{2} v \cdot v$

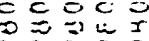

o:

xosñ แกกน

$\therefore \dot{2} \dot{2} \dot{2}$

$x \cdot 2 \pi$

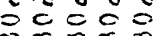

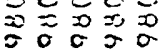

$=0 m m n$

o: ans

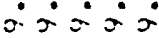

$v \sim \sim v i$

$c 0 \cong 0$

$00 \equiv 0$

i ${ }_{1}=i$

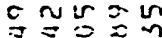

$\dot{i} \dot{i} \dot{i} \dot{\Omega}$

중

vin $v$

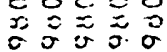

ธேธับับ

๘ேーー

$\varepsilon \sim \sigma \pi=$

$\sigma \div \div$ เ

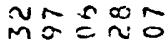

$\sim 0 \subset-\infty$

$\vec{n} \rightarrow-1$ :

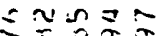
$\therefore \div \div \div$

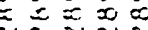

$\mathrm{E}=0 \mathrm{e}=$

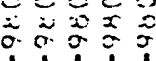

1,11

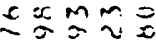

$\therefore \therefore \dot{2} \dot{0}$

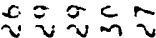

SOOCO

$\begin{array}{llll}0 & 0 & 0 & 0\end{array}$

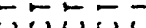

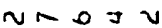

$\therefore \sim c 2 \pi$

$\div \approx \sim ⿻ 上$

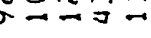

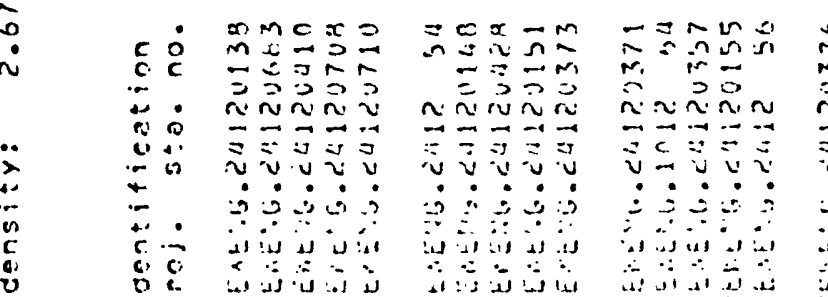

$=x \sim \sim 0$

$=0 \approx-2$ $\therefore \therefore \therefore \therefore$

$\therefore \dot{\vec{v}} \therefore \dot{0}$

กñm

$\div 111$

กNน

$\simeq 2 \div \frac{1}{1}$

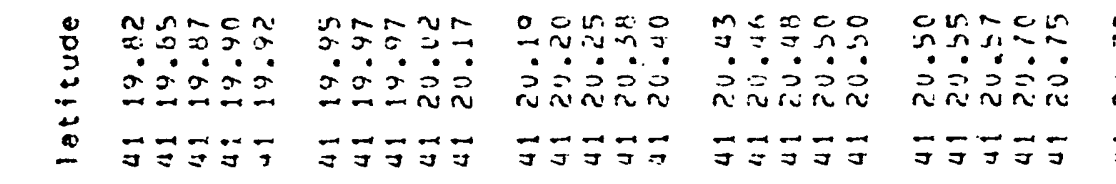

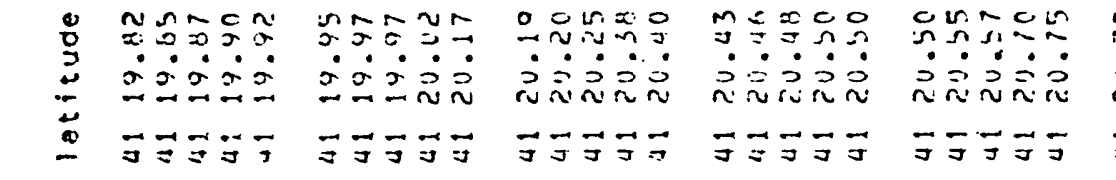

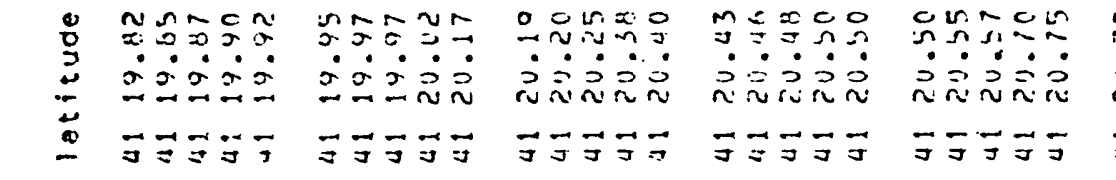

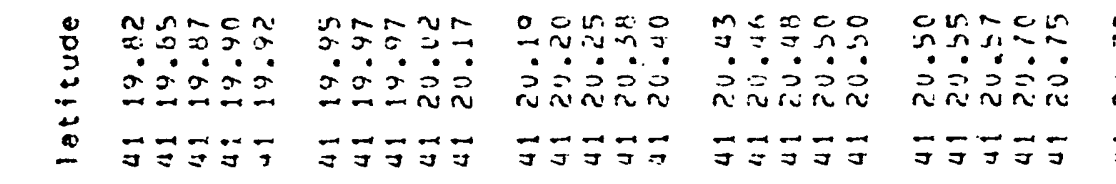

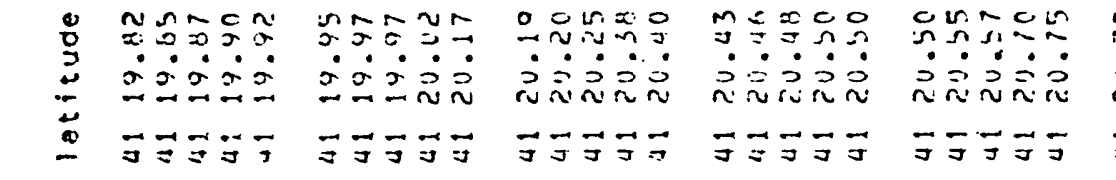

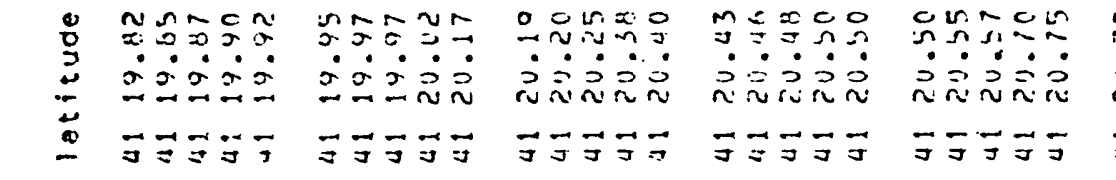

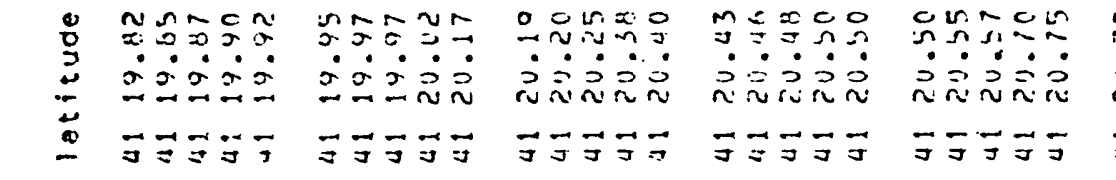

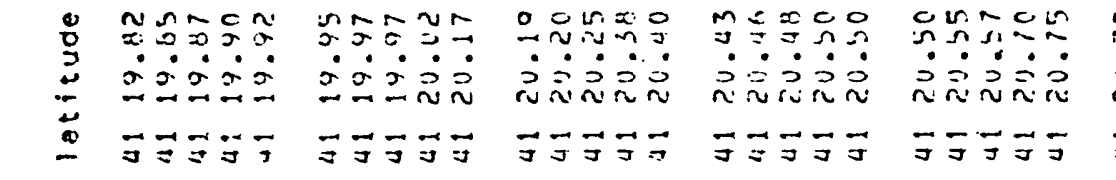

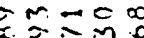

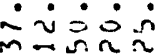

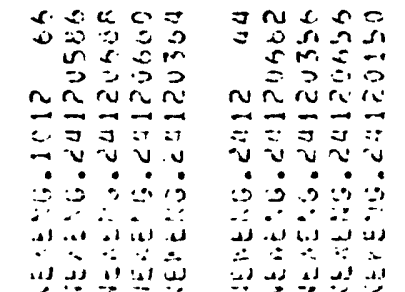

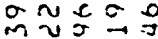

$\therefore \dot{\overrightarrow{7}} \approx \dot{2}$

ur. in.

marin

$\div \div \div \div$

ำกำ응

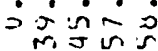

$\sim \sim \sim \sim-$

$\because \div \div \div$

in $\rightarrow \pi \Rightarrow$ in

$s=v v_{0} 0$

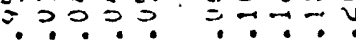

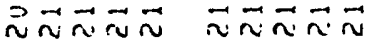

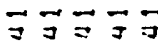

可寻学宁
ง

背:0

$\overrightarrow{2} \overrightarrow{2}$

niven

$\Rightarrow \overrightarrow{9}=7$

ง v v

لـ $\because \therefore 3$

政
จง

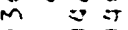

虫仙芯

$\because \vec{\nabla} \because \vec{\nabla}$

v v v v

50 30

הi

A 


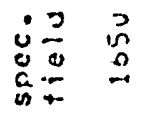

$s$

0
0
0
0
:

$\pi$

ㅇ. 잉영ㄱㅇ

c:

$\div$

:

넝

$\div$

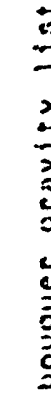

$\begin{array}{ll}5 & 2 \\ 2 & 2 \\ 0 & 2 \\ 5 & 2 \\ 0 & 2 \\ 0 & 2\end{array}$

$\begin{array}{ll}0 & > \\ 0 & 2 \\ 0 & 2 \\ 0 & 2 \\ 4 & 2 \\ 0 & 0\end{array}$

\%

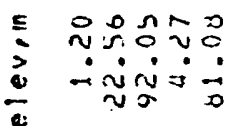

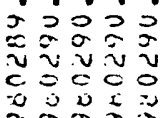

$x=0 \div ?$

क

2

0

: $\therefore \because \dot{\square}=$ வ் $\dot{0} 0$

nn?

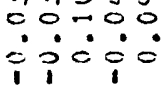

I $0 \cdots 0$

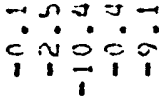

$=00 N v$ $\therefore \dot{0} \dot{0}$ $00 x-v$

万⿻上丨

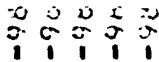

c $=0=\pi$

$n v N=n$

$v=n$

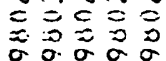

\section{5}

cas $x$ in $\therefore \dot{0} \dot{0}$

유ㄹㅣㅛ ก்

$=\frac{N}{1} \simeq \frac{N}{1}$

\section{$m M \sim N N$}

$1 \div 11$

c. $0: 2 n$ in

บ. in ?

욕ำ

$\vec{\sim} \vec{n} \vec{n} \vec{n}$ $\therefore \therefore \div \div \dot{2}$

ज्ञजज $\vec{\nabla}$ n $\vec{v} \vec{v} \vec{n}$

$\vec{g} \vec{j} \vec{g} \overrightarrow{3}$

93930

둥ㅇ

ำกัด

$\cong 0 \equiv 00$ $\bar{i} \pm \frac{0}{1} i$

$\stackrel{n}{n} \tilde{\Omega}$

ำn幺 $2 \equiv 2 \leq 0$ v $=v_{v} v$ $\begin{array}{llll}1 & 0 & 0 & 0 \\ 0 & 0 & 0 & 0 \\ 0 & 1 & 1\end{array}$

요웅 $\dot{v} \dot{v} \dot{i}$ ทrus

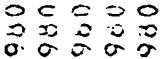

는

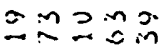

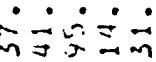

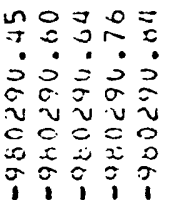

$0 \pi \approx m$ $0=00 \hat{0}$ $v v v v$

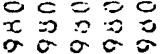

\section{$0 \cong \approx \approx m$

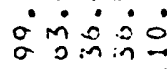 \\ 는}

$\because \stackrel{n}{\infty}: \Omega$ í $\dot{0} \dot{0}$

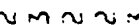
$\div \div \div \frac{1}{1}$

NNIM $\because$ ? ำ $\vec{n} \vec{\sim} \vec{\sim} \vec{s} \vec{n}$ $\vec{\jmath} \vec{\nabla} \vec{j} \overrightarrow{5}$

coron

$c \approx \cdots$
$\Omega \sim$

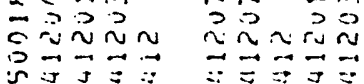
只可 I v

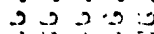
ข:v v v v $\therefore ; \therefore \therefore i$ il $2+0$ is $\therefore \therefore \therefore$ $m=30$ nn $n=1$

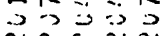

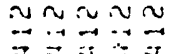

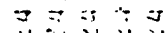
यบ v u v उ;?;? a
NONR

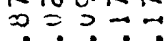
$\vec{c} \vec{a} \ddot{0} \vec{a}$ v c c c 0

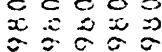

$\hat{i} \hat{i} ; \hat{i}$

든ㄷㄴ

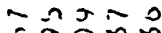

$=\sigma 0 m i n$

$\therefore \dot{0} \ddot{\sim} i \vec{v}$

$n m \infty \leq$ $\dot{0} \dot{0} \dot{0}$ zinno $\approx \approx N \approx n$

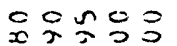

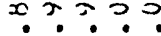
$\vec{\sim} \vec{n} \vec{n} r_{v}$

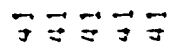

$\Sigma \operatorname{sn} \approx \approx$ $\dot{a} \vec{a}-\dot{0}$ ข้ง v v $x=$

c $\approx 0 \hat{0}=$

$0 \sim x \in c$

oำ $\therefore=\dot{0}=$ v $v \tilde{v}$ 0
0
0
0

जே

crin

vion

요 $n \equiv 0 \sigma$

$\because-\dot{0} \dot{0}$

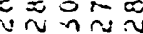

Cㅇㅇㅇㅣ

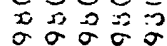

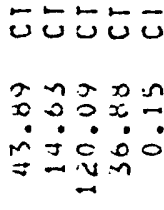

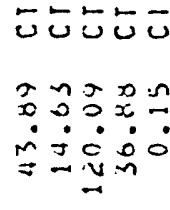

$\Rightarrow \approx \hat{\jmath}=0$

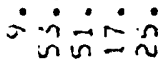

ㅇํㅇㅇㅛ $\therefore \therefore \therefore \dot{0}$ ते $\vec{v} \dot{0}=$

$\approx N=N N$

$1 \% \frac{1}{1}$

$\because N \frac{N}{1}$

summn ?า ?־

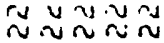

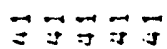

\begin{tabular}{|c|c|}
\hline$\vec{\sim} \vec{\sim} \vec{\sim} \tilde{u}$ & 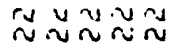 \\
\hline$\vec{J}=\overrightarrow{=} \overrightarrow{\vec{J}}$ & 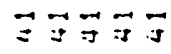 \\
\hline 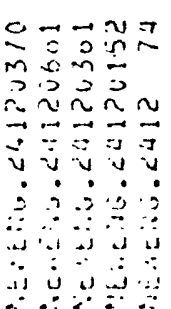 & 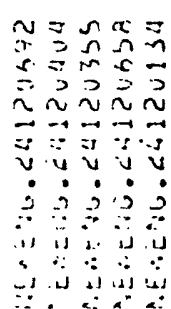 \\
\hline
\end{tabular}

NNo인

เำ ข $v \dot{v} v i$

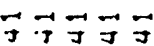

i n $x=00$ $\overrightarrow{2}>2$ $\frac{1}{3} \because \because \because$ 1) v v v ; $;: 30$

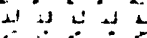

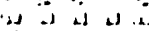

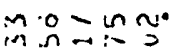
$\because \Rightarrow_{0} \because$

$\dot{\Delta i v-} \dot{0} \dot{0}$

$\ddot{1} \dddot{1} \overrightarrow{1}$

a $\sim 20$

$0 x+$

?.?

$? \div$

$\because \therefore \dot{0}$

M 1111

32333

323

뭉ㅇ

$\therefore \dot{0}=$

$\sim \sim$
0
0

$\hat{n}=000$ ०5000 $00=$

$\vec{\exists} \underset{x}{\sim} \sim$

as $\supset \Rightarrow 0$

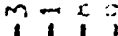

$=\hat{2}=$

$\vec{a} \approx$

$\dot{\sigma}=\dot{v} \dot{v}$

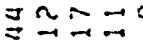

ก⿻上丨刃

$\dot{2} \div \div-1$

v

$=0 x$

$\frac{5}{1} i \frac{x}{2}=$

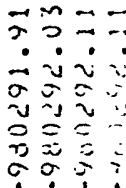

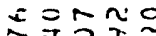

$\dot{\sim} \dot{\vec{z}} \dot{=}$

$x \sim c=\pi$

$\tilde{0} \cong 0 \tilde{0}$

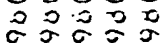

웅: : : $=$

$\dot{j} \dot{0} \dot{0}-$

Nis

$0=0$

分向:

テテั5

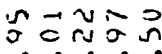

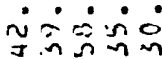

テテら:

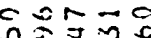

$00 \%$

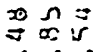
osin:

Mn: $\dot{0}=\dot{\sim} \dot{\vec{\sim}} \dot{\mathrm{in}}$

in $2 \dot{0}$

$m \sim N---\infty \sim \Omega$

$11 \div 11 \% 111$

$\sim M m n G$ งnnn= v่viv

$\ddot{\vec{I}} \overrightarrow{\mathrm{J}} \overrightarrow{\mathrm{J}}$

$0 x$ 舟

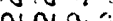

$\overline{2} \overrightarrow{2}$

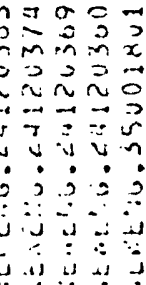

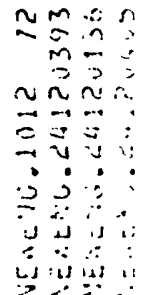


no onm

mingn

$\therefore \dot{m_{1}} \dot{0}$

m Mrs

i-نं:

$i$

- 00300

(5)

:

$\begin{array}{ll}c \\ 0 \\ 2 & 2 \\ 0 & 2 \\ 4 & 2 \\ 4 & 2 \\ 2 & 0 \\ 2 & 2 \\ 0 & 0 \\ 0 & 0\end{array}$

\section{जत्र可}

$\therefore 0000$

oc $\ln _{0} \overrightarrow{0}$ $\because=\dot{m}=\dot{m}$

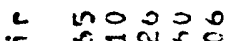

niñn

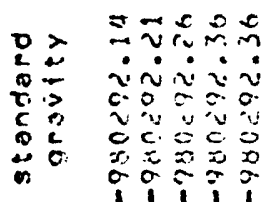

위

Nom in

$\begin{array}{ll}0 & 2 \\ 4 & 2 \\ 2 & = \\ 0 & 0 \\ 0 & 2 \\ 0 & 5\end{array}$

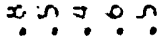

$\rightarrow \sim-j=\overrightarrow{0}$

vin

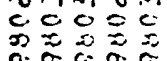

\& ธேธ์ธั

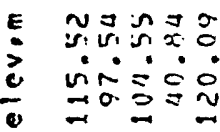

:

$\frac{0}{2}$

and in? $m-m \sim 0$.

$\sim m \sim m$ $\div \div \div \div$

II: 2 MO $\dot{\sim} \dot{\sim} \dot{v} \dot{v}$ 〜NCa: ज守宁可

c $\rightarrow$ in

$\therefore x \approx \sigma$

$v \sim v v v$

$-=-$

똑곡

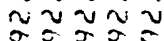

o 00

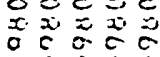

r. $=x$ un

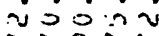

证证

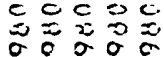

ธேับ5

$\sin x=$

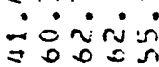

$\Leftrightarrow \overrightarrow{2} \mathrm{~N}=$

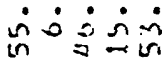

$=22=$

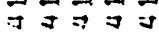

nnms

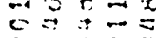

HEn $\approx m$ rim $\therefore \vec{n} \vec{\sim} \ddot{0}$

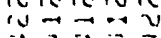

$\because \vec{v}) \bar{v}$

$\therefore \therefore \therefore:$

لـ
$\Xi 001$

ivنs

c

$\because \sim \ddot{n}=\dot{2}$

$\Rightarrow m$ 的

일ㄹ

ํํำ

o.ỹn

20303

$\dot{0} \dot{0} \dot{0}$

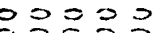

IN $=0$ 이웅

$2=200$ $\therefore 0^{\circ} \circ 0^{\circ}$

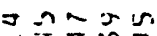

$\because \because \therefore M$

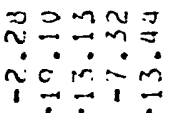

$=0200$
$\approx \therefore m x: 0$

$\ln 7 x$ $\because \sigma \div 5$ $\dot{m} \dot{m}=\dot{c}$

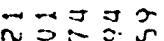
$\dot{0} \dot{\sim} \dot{0}$

$n=m-0$ $\because \div \dot{0}$ ก $n$ in

$m=\hat{n} \approx$ ขinji

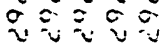
OOC $=$

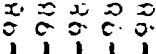

$x \approx \bar{x}=$ $\dot{v} \dot{v}-\dot{v} \dot{v}$ $2 \approx c 5$ 至余:

$0=2=i$ $?=?-\overrightarrow{1}$

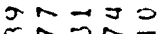
ขขน $\equiv c 0 c$

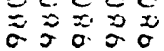

பேபே

00505

$0 ? \div ?$ 에묘

ขก $\sim \sim \sim$

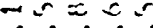
$\dot{c} \dot{x}=\dot{x}$

$\vec{\sigma}=\vec{z}=$ $\dot{0} \dot{v} \dot{0}$ $\approx \approx \sigma=\sigma$ uv v v v

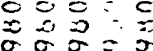

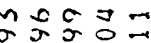
$\sim \dot{v} \dot{v} v$ 20 5 $000=0$ $=0000$ i 11 ;

$M N M \infty F$ ของ กNล ปี⿻一㇉丶 ำ $\approx \approx \equiv \approx$

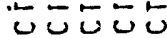

-OMJO

ก $\because n O$

$0000=$

욜 $\vec{x} \vec{c}$ $\dot{x} \dot{0} \dot{0}$

ภच ปัต $0 N \div \div \frac{1}{1}$

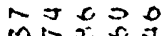
$\therefore \dot{\sim} \dot{0}$ กั

$\because 0 \approx=$ $\dot{0} \sim \tilde{N}=\dot{0}$

뭄 $\vec{x}$ Dimi:

$m=m=$ - ! ! ? लि

ำนำ $\dot{m} \dot{n} \dot{n} \dot{n}$ $20 \%$

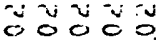

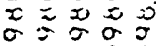

$\approx r=\nabla=0$ $\because \div \div \dot{0}$ vrin $\begin{array}{lllll}0 & 1 & 0 & 0 \\ 0 & 0 & 0 & 0 \\ 0 & 0 & 0 & 0 & 0\end{array}$ $\sigma \sigma=\sigma \sigma$

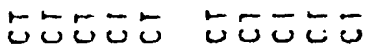
$5=0=$

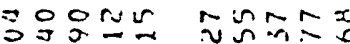

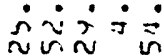

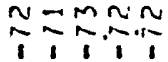
$\dot{\vec{v}} \dot{\sim} \dot{=} \dot{s}$

$n \sim n n$

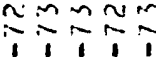

$\therefore 0 \vec{c} 0$ is

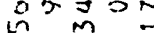

annm $\div \div \frac{1}{1} \div$

$\bar{\sigma} \sim=\ln c$ $\sigma 心 j=$ ivivi rurina

$0 x=r x$ $\exists=-7$

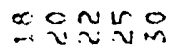
- ? ! กินึก๊ก 可可宁

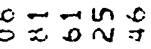

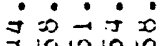
NDN $\approx$ $2 \div 2 \div \div$

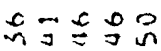
$\dot{m} \dot{n} \dot{n} \dot{n} \dot{n}$ พnNก

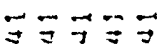

c: $s-m$ in $5-0 j$ in

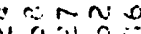
1is in $\Rightarrow \Rightarrow \overrightarrow{3}$ 2arn $\because \because \because$ บง v v $\therefore \therefore \therefore$

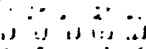

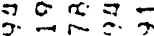
$\therefore=s i n$ $35: 30$ Nㅡㄴ $\vec{v} \ddot{v}: \ddot{v}$ v v v v $\therefore \because, \therefore ;$

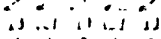

oñ $\dot{m} \dot{i n} \dot{\sim} \dot{m}$

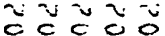

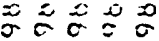
; $15 \%$

orogin

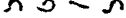
$\dot{m} \dot{0} \dot{\sigma} \dot{v}$ $\pi \approx 00$ $\sim v v v$

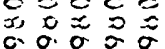

$00 \overrightarrow{0} \sim$

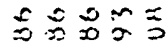
$\therefore \dot{n} \dot{\sim}=$ c. 000 Oc $=0$ $\begin{array}{cccc}20 & 0 & 0 & 0 \\ 0 & 0 & 0 & 0\end{array}$ 11111

x०๊̃c $\therefore \therefore \therefore$ $\bar{n} \approx x=$ $n \sim v v=$ $\begin{array}{llll}0 & 0 & 0 & 0\end{array}$

ஸ5๋テ

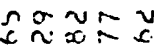
$\stackrel{n}{n} \sim \sim \sim$

cnㅐ용

c. 0005 $\dot{\jmath} \dot{i} \dot{\sim} \dot{0}$ $\cong N \div \frac{1}{1} \div \div \frac{1}{1} \div 1$

N $\tilde{D}_{0}= \pm 0$ inisin

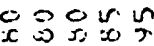
nruñon. 可可可

J - m in os un- 00 ง N N iv $\vec{*} \cdot \vec{a} \cdot \vec{a}$ v v $v$ $\therefore \quad \therefore \dot{0}$ (i) 210 is 


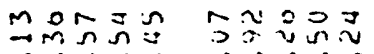

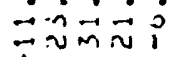

วับำ

$\tilde{n}^{n} \div \frac{0}{1} !$

路:ภร

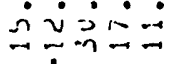

บำกำ

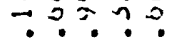

ืํำำ

$\therefore \therefore i \div$

i̊요

丁

-

000.

33333

○ं0்

33339

$\therefore 0$ ¿ं०

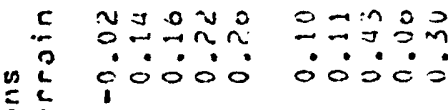

눈

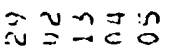

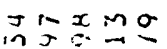

$\therefore \therefore \therefore$

ni=n

$\therefore 000$

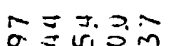

$0: 5 ? m$

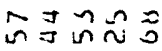

$\because \div \simeq \dddot{1} \div$

anis

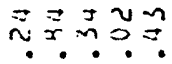

$\because \div \frac{1}{1}$

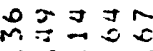

$\exists \tau x \simeq \Omega$

nis 0 m

$\dot{\vec{B}} \dot{0} \dot{0}=$

$=20 \Sigma \overline{1}$

$\dot{\sim} \dot{\sim} \dot{0} \dot{c}$

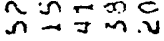

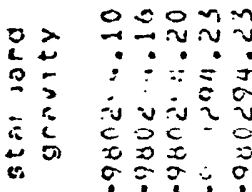

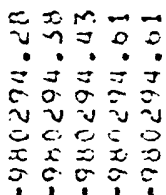

$\equiv=000$

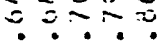

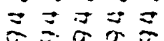

证岛

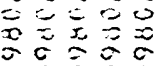

15350

동․․

告

ans

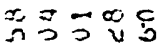

F $\vec{E}: ?$

cᄃㅇ․

$\dot{i} \dot{2} \dot{v}$

중

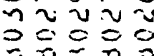

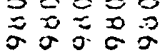

:

$\frac{E}{2}$

๖๖こธこ

ติ $\sim 0$

$\because 0 \div \div$

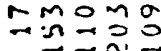

๖ヒัธง

约的员。

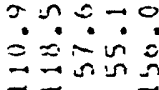

ONMm

$\dot{0} \dot{0}$

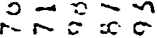

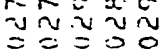

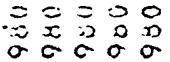

노

n-mnn

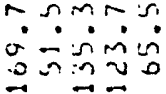

$\sigma \sim_{0} \sim 0$

กำกำ

\section{은}

$\dot{0} \dot{0} \dot{0}=$

$\dot{\sigma} \dot{\vec{j} \dot{\nabla} \dot{m}}$

-mnima

$\because \sim \sim-N$

$\div \div \div \%$

$\div \div \div \div$

0
0
3
3
2

ocminis

$\infty$ เ $0=$

- i.j.

บำ?

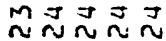

只忌芯忌

$\vec{\exists} \because コ \vec{\exists}$

吉吉す

u

2

ㄷㅇㅇ

$\geq 5$

n

$3:=$

$\therefore$ is

a 2 के

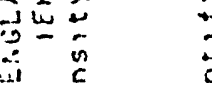

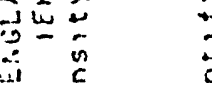

is

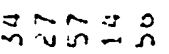

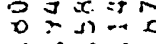

폭

$\dot{2} \dot{2} \dot{0}$

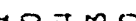

w $\rightarrow=n$

ทำ

$\dot{0}=\dot{1} \ddot{1}$

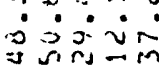

영여응

0ं0ं0.

33333

¿0ं0.

m0류

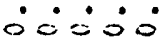

omiag

n. 2000 ¿0000

$\stackrel{\sim}{0} \overrightarrow{0} \tilde{n}$ ¿ல்0்

ว포에 نंक्षित

ヘேテテ 1:

$\stackrel{5}{=} \cong \cong$ जिं்

$\because \sim \sim \sim 00$

$P \sim=0 N$

s.

$=\vec{i} \vec{i} \tilde{i}$

กำテへ

añ $=x$

它守心

$\stackrel{x}{\sim} \dot{0} \dot{0}$

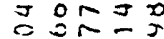

$\dot{\sim} \dot{-\varepsilon \dot{c}}$

몬

$\dot{n}=\dot{i}=$

ㄱำ

บก

$00: 3$

$\dot{\therefore} \dot{\circ}$

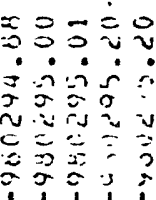

$\Rightarrow \approx \sim \vec{*}$

i $:$ in

认 $v u$

$\begin{array}{llll}0 & 0 & 0 & 0 \\ 0 & 0 & 0 & 0\end{array}$

c0

$\therefore \approx \hat{n} \tilde{\sigma}$

$\because c \bar{c} \dot{v}$

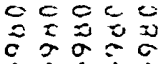

$m \vec{a} \approx \cdots$

m

$\dot{0} \dot{0} \dot{0} \dot{0} \dot{0} \dot{0}$

원: i

oc

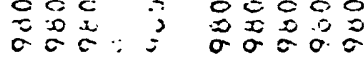

1,111

의의

$\dot{0} \dot{0} \dot{0}$

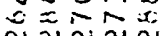

$\simeq \widetilde{v} \widetilde{v}$

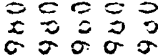

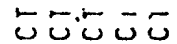

5

toxin

$0=x \leqslant$ in

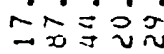

$\therefore \geq \infty$

$m \sigma=00$

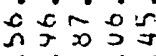

テังับั

$\because 0 \hat{0}$

$\because 0 ? \Omega x$

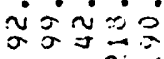

$M M=0 \backsim$

$\min =0$

西:

更

की i i

$x \sim \because \pi m$

$\dot{i}: \dot{0} \dot{0}$

$x=0$

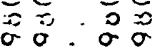

שับ

nกำ 0

$\because x \sigma 0 n$

슴

을 $\approx$ 幽金:

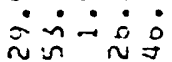

$m=m m \simeq$

ninon

in $\ln y=$

录芯芯

可寻可

nno $\sigma$ in is $2 \times 1$ $\therefore \dot{0} \dot{\sim}$

我 $\therefore \dot{0} \dot{0}$

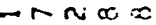

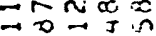

음동 $\ddot{n} \dot{0}=\dot{m}$

$\div \div \div \div$

Un: $\because 00 m$

$\vec{\exists}=\vec{J} \vec{\exists}$

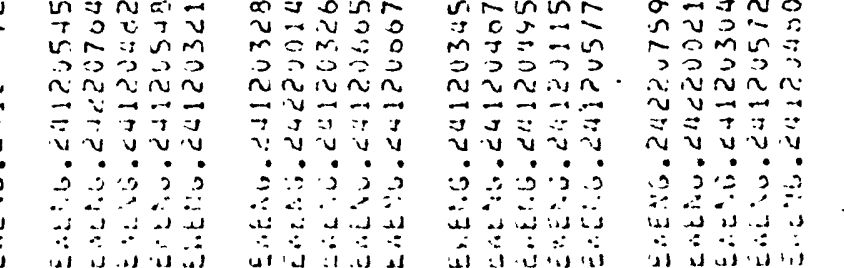

$\therefore \therefore \therefore \Omega$

$=E \cdot 0 c$

m

$n \sim-\sim N$

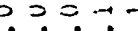
$\hat{n} \Omega \Omega$

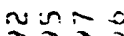

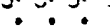

กิกร

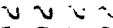

正要

11

a Non

$\dot{0} \dot{0} \dot{0}$

$=000$

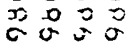

ヒこここ

$\underset{0}{0} \rightarrow \mathrm{c}: 2$

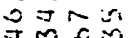

$\rightarrow$

$\Rightarrow 0.5$

$\dot{0} \dot{0} \dot{0}$

vmm

$\hat{1} \hat{1}$

$\infty 0$ 수

- $\dot{0}$

ñus.

$\vec{\exists} \ddot{I} \vec{J}$

In 0 in 0

3202

N心N

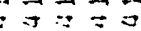

ง v v v

$\therefore \therefore ;$

נה

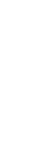

$=0.7 \%$ m 2 . 2 . Nnn $\vec{\nabla}: \vec{\because}$ $v-j$ $\therefore \dot{3} ;$ 넌 
$=0$

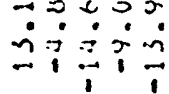

$n \sigma=0$

$\therefore: \therefore$

. $111 \frac{1}{1}$

30898

0ं் 0 0

$\sim \cap \rightarrow N=$

กำ웅

00000

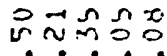

xim

$=n 0 \ldots$

$\because \therefore: \sigma m$

i் $\dot{\sim} \dot{\vec{n}}$

:

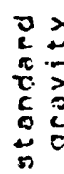

$\exists \infty \infty \vec{\sim} \approx$

$\dot{0} \dot{0} \dot{0}$

ขีนี้

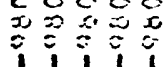

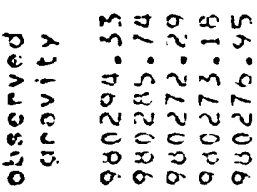

ถั

;

ธ5ธ25

m $n \ddot{2} \approx n$

ㅁํำ총

$\bar{c}$

coinin $x$ ว.? ? : =

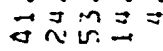

$\sim N-N N$ กิ11 ü

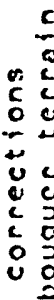

\begin{tabular}{l}
1 \\
0 \\
0 \\
0 \\
\hdashline \\
0 \\
0 \\
0 \\
0
\end{tabular}

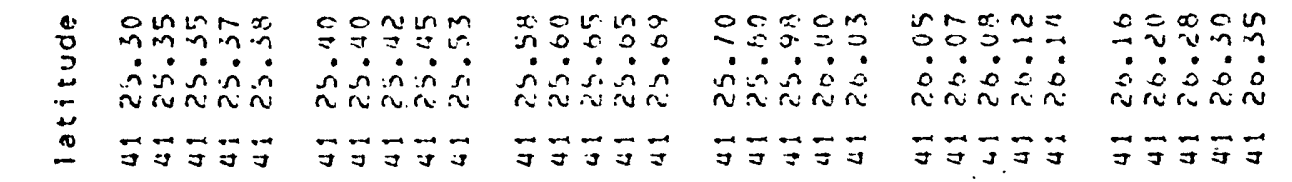

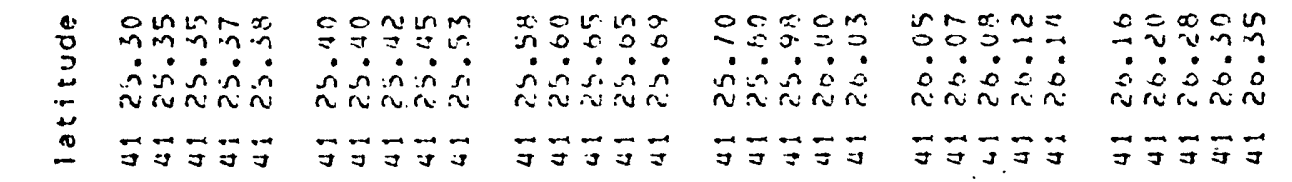

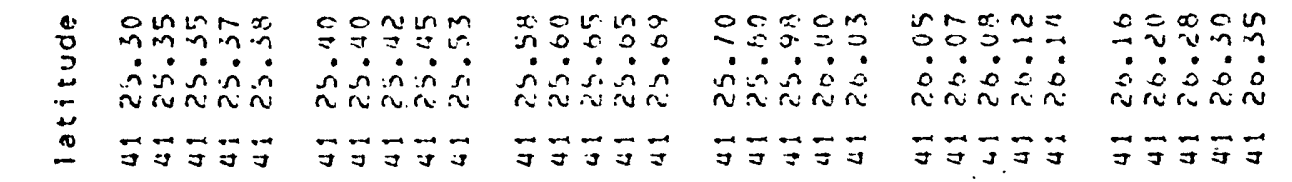

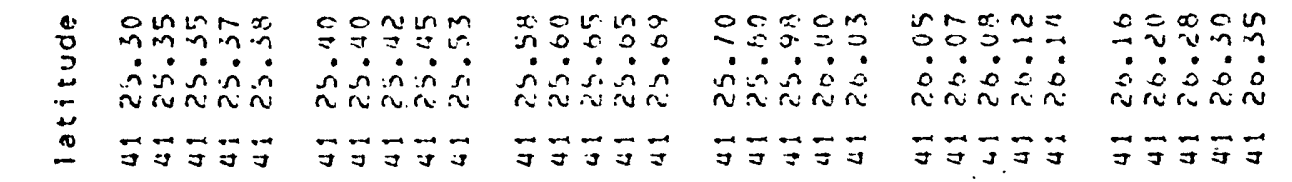

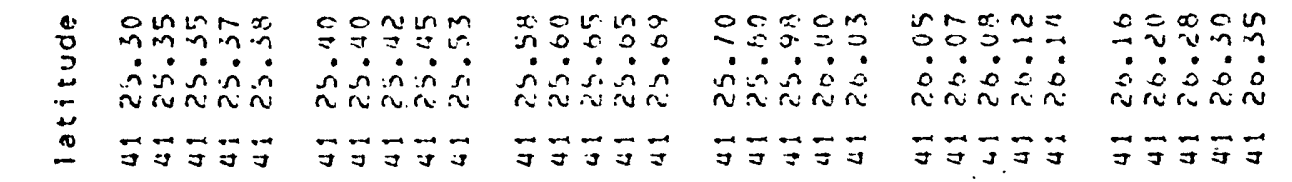

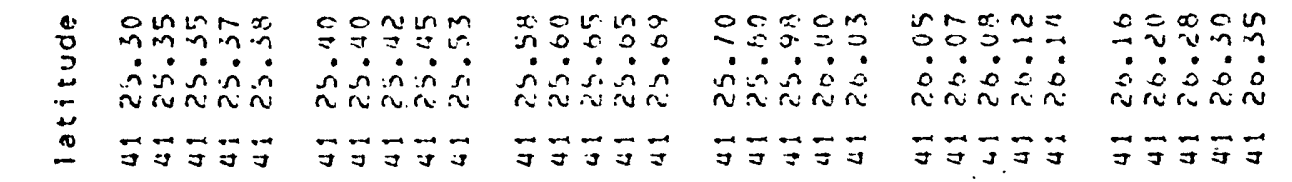

$\approx$ 주요

on $\Rightarrow$

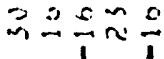

GNAo in

$\because 750$.

ㄱำ证

웅ㄹㅇㅇㅇ

¿ذंबं

ํำำㅇํㅇ

$\therefore 0<00$

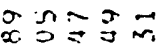

$m \ddot{\Xi} \ddot{1}$

№ 00

En vin in

동ํㅇ

viñ

$\dot{0} \dot{0} \dot{0} 0$

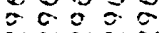

co름

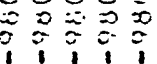

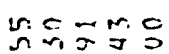

$\dot{\sim} \therefore \dot{n} \dot{0}$

得的令

c. yudu

ํำ 20 $\sim \dot{x} \dot{0}=$

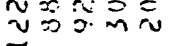

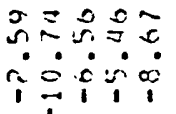

แn 020 $\therefore \dot{\therefore} \dot{\sim} \dot{\sim}$

응으으응 $\therefore \dot{0} \dot{0}$

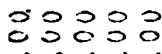
¿cं0.

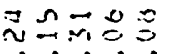

ํํำำำ ¿cं0.

¿ंड்

ตก๊̃อ

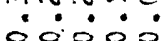

웅료응 $09=3$ ○ण0ं0

30030 $=3059$ ¿0ं0.

옹옹ㅇㅇㅇ 0000

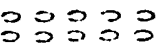
0000

Nos: No $\because-m$

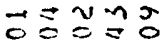
c0000

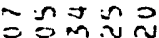
¿000்

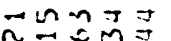

.

Mำ

포용

$\simeq \sim \underset{0}{0}$

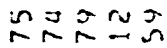

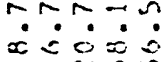

릉ํำ

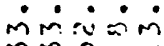

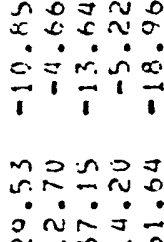

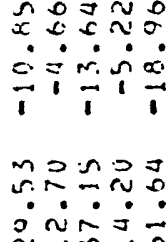

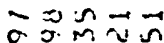

จinisis

I

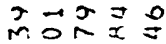
$\because \because \approx 5$

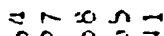

กกก

$m= \pm x 0$

$\pi 50 x \geqslant$ $\circ \sigma \underline{0}$

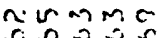

용 $\dot{\therefore} \dot{0} \dot{0}$

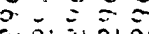

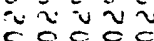
$0 \equiv x x$ $1 i$

nemon $\because \because \because \because$ $\ddot{1} \dot{2} \dot{n}$

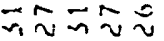
잉에 0

$\sim \stackrel{\sim}{\sim} m \tilde{m}$ $\dot{0} \therefore \therefore$

$-5000$

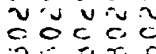

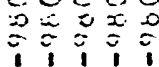

ñm

c. $=0$

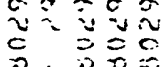

$\therefore \therefore 30$

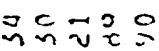

$\dot{0} \dot{0} \dot{0}$

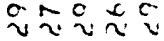

음ㅊㅇㅛ

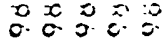

$m-J P \sigma$

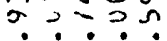

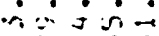

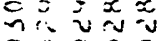

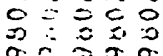

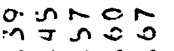

$M ะ \cong \cong \Xi$

- 0.

$000=0$

v v v

0

1:

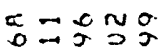

$\dot{0} \dot{\sim} \dot{0}$

列记

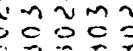

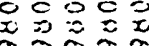

运立定

$\sigma=0$

20.2

$0 x x y$

१ิ

- 000

ก บ $>$ ก

vin $x$

$\hat{v} \approx \bar{v} \tau$

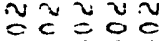

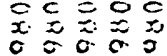$$
\text { (1) }
$$

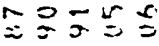

$\therefore \sim ⿻ \dot{x} \dot{x}$

议语

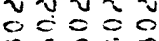

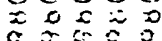

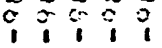

$=0 n \pi 2$ $\dot{0} \dot{0} \cdot \dot{0}$ 웜

c.

c引히

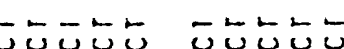

$\div 2 \div 20$

munimin

๖ここちテ

ธேบับับ

カேトே

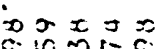

$\rightarrow$ in 0 N

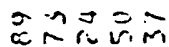

$\Leftrightarrow 30 \therefore$

$\therefore \dot{0} \dot{0}$

minnim

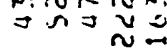

テこちこう

$\vec{x} \vec{x} \vec{x}$

$x \vec{x} \vec{x}=$

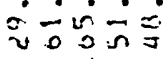

ט $=50$

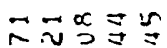

iñ $\dot{n} \dot{\sim}$

na:

o o on N N

$30,20 \quad r=-\pi n=$

نिm $\dot{i} \dot{n}$

$\therefore \dot{\sim} \dot{0} \dot{0} \dot{\sim}$

ํำM

ก๊man

$\sim \sim N \sim m$

$\cong \sigma \overrightarrow{0}=$

$\approx \pi x=5$

$\therefore \dot{v} \dot{\sim} \dot{0}=$

mrimna

$\vec{\nabla}=0$

กงวm

$\dot{m} \dot{0} \dot{0} \dot{0}$

$\cong n N \cong m$
- $\cos 25$ -

프N

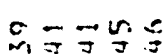
$\dot{0} \dot{0} \dot{0} \dot{0}$ $n \therefore i n$ $m=\dot{0} \dot{0}$

于宁学

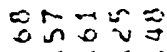
它这完向 - $2 n \pi$ $1 \% \div \%$

$\forall=\overline{5} 0$ $\dot{0} \dot{0} \dot{0}$ $\vec{\exists} \vec{\exists} \vec{\exists}$

西

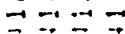
政焉 $\therefore \dot{3} \dot{3}$ נד is

$=c m \sim x$ जलM

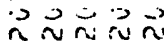
는

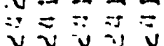
$\therefore \therefore ;$

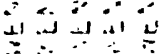
با 


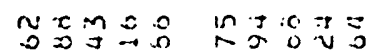

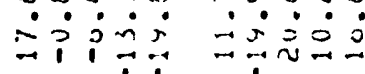

$\infty \div \sim \overline{0}$

?.?

$2000 \div$

$\ln 00 \sin$

$\dot{\dot{t}} \dot{\mathrm{m}} \dot{\mathrm{i}} \mathrm{i}$

33930

33300

$\therefore 0000$

¿0ं0:

a

ن

Nก⿻上丨

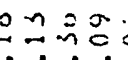

¿ं00

05000

os $\sim \sim 0$

$\because \because \cdots x$.

$5 x=30$

$\vec{i} \div \div$

i०ं

y $10 \div 0$

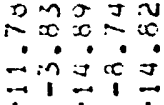

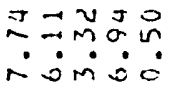

ก⿻:

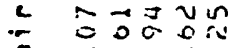

$x \simeq M D$

iिं

- $\dot{0}$

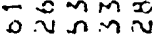

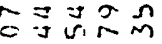

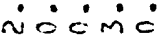

ํํำㅇำ

$\therefore \dot{0}: \dot{0}$

$\therefore$

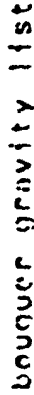

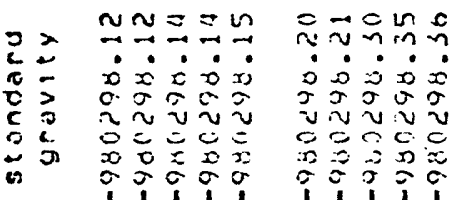

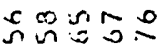
$\dot{0} \dot{0} \dot{0}=\dot{0}$

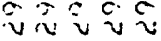

00

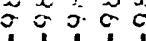

$m \approx 000$

$\begin{array}{ll}0 & 2 \\ 8 & 2 \\ 2 & 2 \\ 2 & 2 \\ 0 & 0 \\ 0 & 2 \\ 0 & 2\end{array}$

$m=0=0$

Non $N=$

ñs

avinu

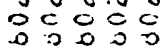

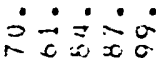

$\forall v \approx v$

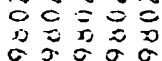

1111

$50 \cong \approx 5$

$\dot{0} \dot{0} \dot{0}$

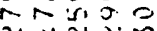

$\sim \cup v \dot{0}$

$\begin{array}{ccc}2 & 0 & 0 \\ 0 & 0 & 0\end{array}$

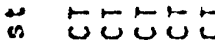

ヒここちち

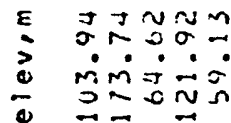

$02 \simeq \pi n$

$\propto 2=n x$

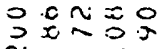

๖ธธธธ

Jn-

$\sigma x m \because \because$

भim

ก๊o

บำ?

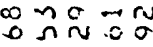

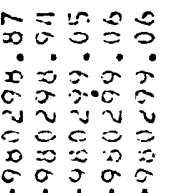

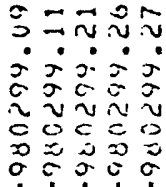

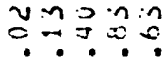

mani

$\overrightarrow{1} \overrightarrow{1}$

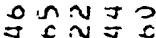

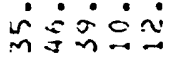

$2 \pi \cdot 000$

$\therefore \therefore \dot{0} \dot{0}$

ㄴvำ

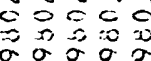

$\approx 0 \simeq \approx 0$

$\dot{0} \dot{0} \dot{0}$

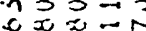

v $\sim \sim v$

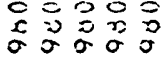

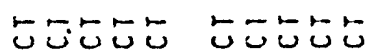

$-\infty 0 \mathrm{NJ}$

$\because \because \dot{m} \dot{0}$

añm

$x \approx 0 m$ in

$\infty \sim n=\varepsilon$

$\because \exists \sim x: 0$

$\stackrel{3}{3}$

$\therefore \approx \overrightarrow{7}=\overrightarrow{0}$

ง ข $=\pi$ $\therefore \dot{0} \approx \dot{\sigma}$

๙

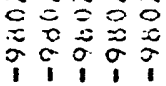

ำกำ

$\therefore \dot{0} \dot{0}$

宁运完它

ป̃

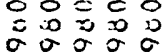

ธธธธธ

$\ddot{v} \vec{n} v$

$n: N r=$

$\tilde{N}=\underset{0}{n}$

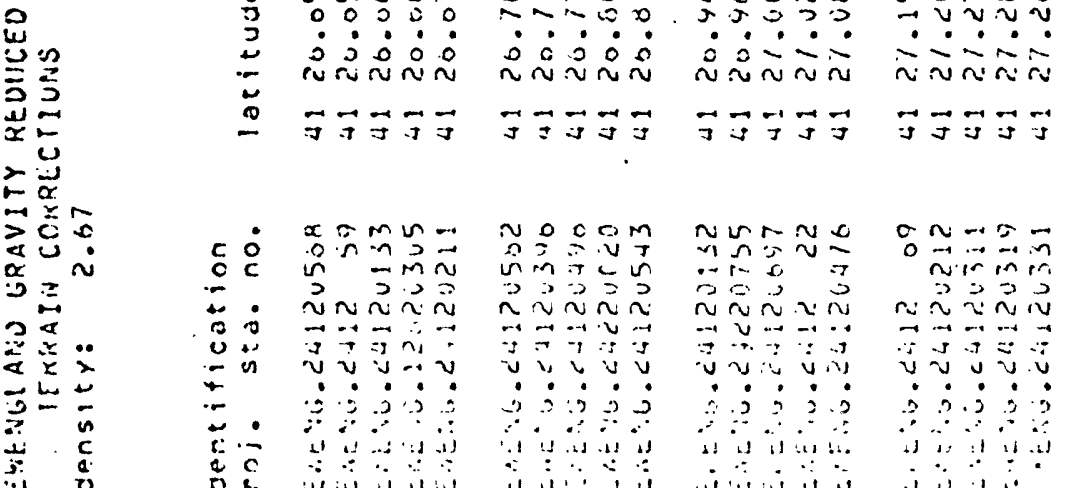

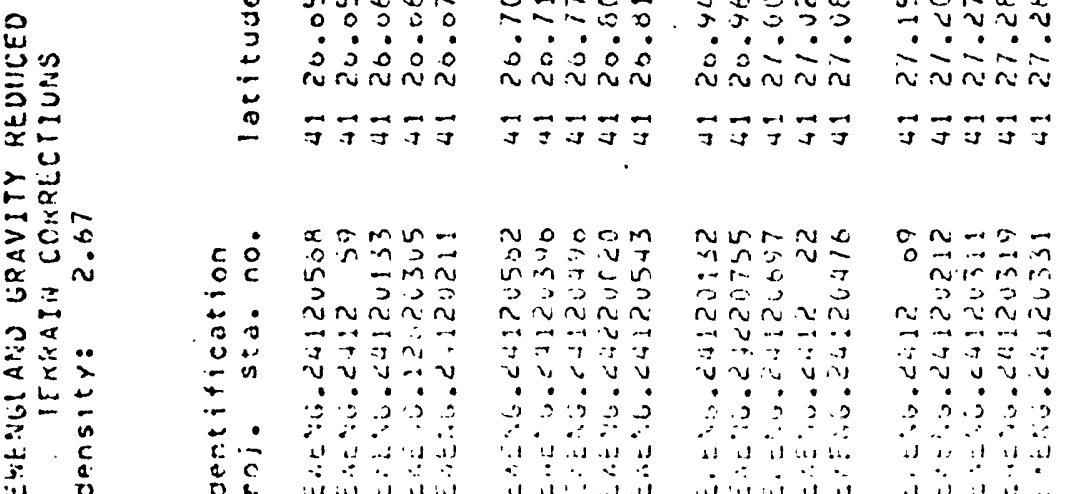

\section{c}

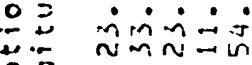

$\dot{0} \dot{0} \dot{0}$

in ing no

0.007 .0

N 2005

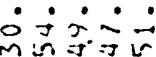

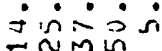

$\hat{n} \mathbb{i}=\vec{\exists}$

등

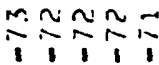

mNMNm

$\div \% \div \%$

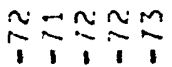

$\sim-m \sim n$

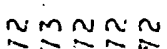

$\cong N \cong \pi \frac{1}{1}$

$1 \div 1 \%$

$0 \approx N 0 \overrightarrow{0}$

in in o o

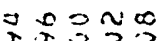

Cथ - :

ก̃̃ñ

ज菏
욱 $\dot{0} \dot{\vec{j}} \dot{0}$

MNNMM

$1 \% 1 \%$

$\cong \min \alpha$ กิ̃ก̃ñ 可可焉

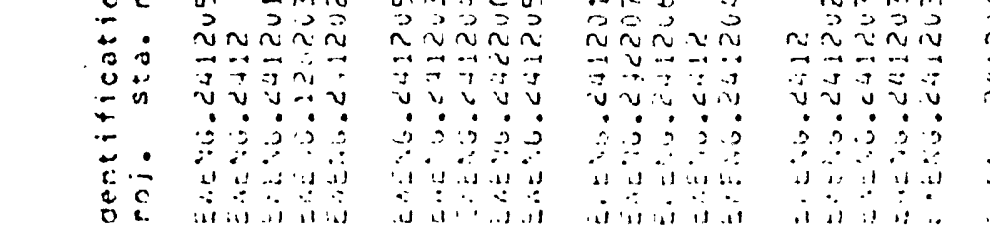
s in 两 20

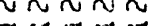

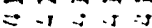
ป v v.v v $\Rightarrow 3: 20$ $\because \therefore$ j
ヘ

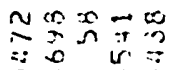
$\begin{array}{lll}\because & 0 \\ \sim & 0 & 3\end{array}$ NN논

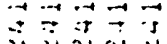
$\ddot{v} \dot{v} u v$ $\therefore \therefore 5 ;$

$=c \approx N$ $\therefore 0$ วก

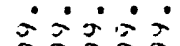
讨证 $\therefore c=00$ $\approx 2000$

J. $\therefore \sim \sim m \dot{x}$

cmำ 它血品立

젠?

$\dot{\alpha} \dot{0} \dot{\circ}$

in $M \equiv 00$

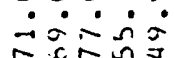
ฟบv $v$ OO

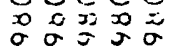

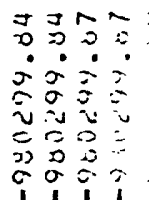

它包包

以っコルコ c $30 \pi$. - $\dot{0} \dot{0} \dot{0}$

$\ddot{x}=\tilde{s}$

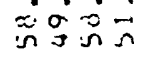

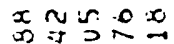
$\dot{0} \dot{\sim} \dot{\sim} \tilde{\sim}$ $n \rightarrow m-N$

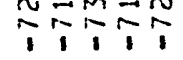

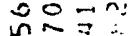

$\dot{0} \dot{\mathbf{I}} \dot{\mathbf{2}}$

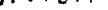

$n m-n$

$1 \% 1$

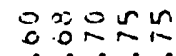
$\therefore \therefore \therefore-$

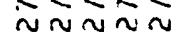

o on

可䒚可

$\therefore \therefore$.

iñ

$\vec{\nabla}=\vec{g}$

$\sin$

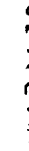

政 


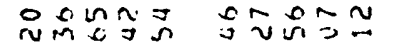

$\dot{0} \dot{\sim} \dot{\sim} \dot{\sim} \dot{\sim} \dot{\sim}$

$\stackrel{0}{\sim} \sim \tilde{N} \sim \tilde{N} \sim \tilde{N}$

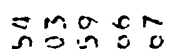

injunis

ninc. $=\stackrel{\alpha}{\sim}$

$\dot{\sim} \dot{\sim} \dot{0} \dot{0}$

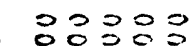

$\therefore \dot{0} 0 \dot{0}$

$30=$

00000

$0300=$

$\therefore \therefore \circ \dot{0}=$

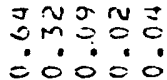

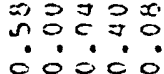

m证:

공요영

¿ $\dot{0} \dot{0} 0$

웅?ㅛ? 3

$\therefore \dot{0} \dot{0}$

$3383 ?$

$\dot{0} \dot{0} \dot{\circ}$

30303

.

00000

영여의

$\therefore \therefore$

20303

00000

00000 $m=0=0$ กับง?ำ. थิ

sกก⿻m c:??:?

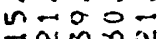

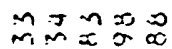
$\therefore \dot{0} \dot{0}$

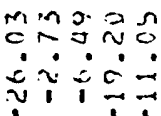

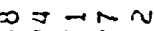
$\because=12 \pi$ mos 0

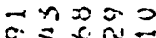

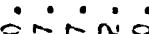
Nㅡㅁำ

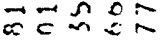
añ $\dot{0}$

$c \simeq 2 n=$ $\therefore \therefore \therefore \dot{0}$

$\sin n$

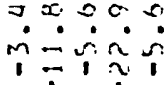

Joñ onmm? $\because \frac{0}{1} \div$

on 0 in ำ $2 m=m$ Dinis miña กก约

$\Rightarrow \exists 0 \approx=$

이요

눈?

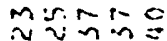

$\Xi=\exists$ ง

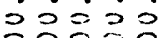
이의

$\therefore \equiv 000$ i

$\begin{array}{ll}1 & 0 \\ 0 & 0\end{array}$

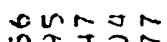

cis

ทำว?

๓

m实

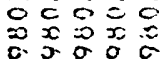

$0=000$

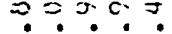

in:

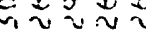

$c=00$

¿ें $\dot{0}$

은둔

oc

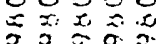

\% $10 \% ?$

ron

$0=0 \div$

ว $\simeq \approx 0$

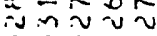

$\begin{array}{llll}0 & 0 \\ 0 & 0 & 0 & 0 \\ 0 & 0 & 0 & 0\end{array}$

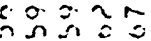

¿ذं0:

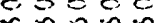

o o c c =

$x_{1} x=2$

ci 19

$x m \sim=0$

ㄱำ

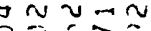

$v v_{1} \sim v$

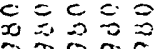

西家

ธังธั

๖ธここう

รับธธง

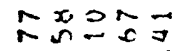

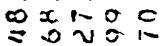

$\approx \approx \approx \approx 0$

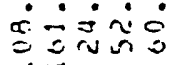

Noon

$\approx C m=s$

20 in 00

$\pi v$ in 0 o

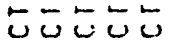

$\hat{0} \approx \vec{z} \geq \vec{x}=$

$\therefore \dot{0} \dot{0}$

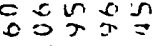

$5: 200$

$\tan 0 m=$

腾亏宁宁

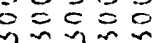

$\therefore 00 c$

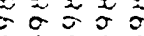

주요 $\sim \dot{0} \dot{x}$

元 $\approx v$

$\begin{array}{lll}c & c & 0 \\ 20 & 0 & 0 \\ 0 & 0 & 0\end{array}$

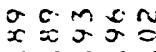

j引룽

inc in 0

co $000 \mathrm{c}$

2. $\begin{array}{llll}0 & 0 & 0 & 0 \\ 0 & 0 & 0\end{array}$

अ $19 \% ?$

$m \sim \sigma=c$

m?

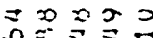

vivum

$\begin{array}{llll}0 & 0 & 0 \\ 0 & 0 & 0 & 0 \\ 0 & 0 & 0 & 0\end{array}$

テここご

รก旅

$=\because 0.5$

OMO00

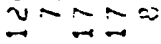

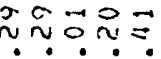
sin $\sin =$

1,13

진 $\therefore \therefore x=$

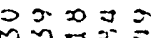

$\ddot{v} \dot{x} \dot{0} \dot{0}$

$\sigma \tilde{2} \approx \approx$

$\dot{0} \dot{0} \dot{=}$

(1)

我宁气

$\therefore \therefore \therefore \therefore$

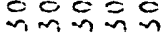

c $\mathrm{C}=\mathrm{C}$

कव 0 व

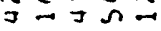

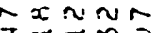

․․

वे.

u $u v$

$c 0000$

$\begin{array}{llll}0 & 0 & 0 & 0 \\ 0 & 0 & 0 & 0 \\ 0 & 0 & 0 & 0\end{array}$

55055

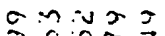

00 n.

$\dot{x} \overrightarrow{0} \dot{v} \dot{\Omega}$

mm $=0$

inis

$\sim \Omega n \leq$

कón

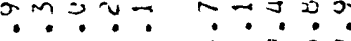

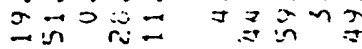

$c=\dot{x} 0=$

ormov

in $\dot{n} \dot{v} \dot{0}$

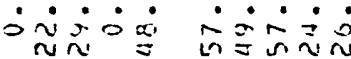

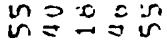

mrinn

n.

man

$\sim N \rightarrow m=$

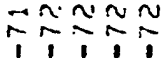

momin

-

$\because \ddot{M} \overrightarrow{0} \dot{0}$

Nonm

$\pi=1 \% \frac{1}{21}$

$\sim c: 5 m$ $\dot{0} \dot{0} \dot{0}$ $\sim 20$ 品

$m M M \simeq \approx$

$\because \% \overline{1}$

Drunt rocona

c: $C \min \sigma$

ก ภ ก

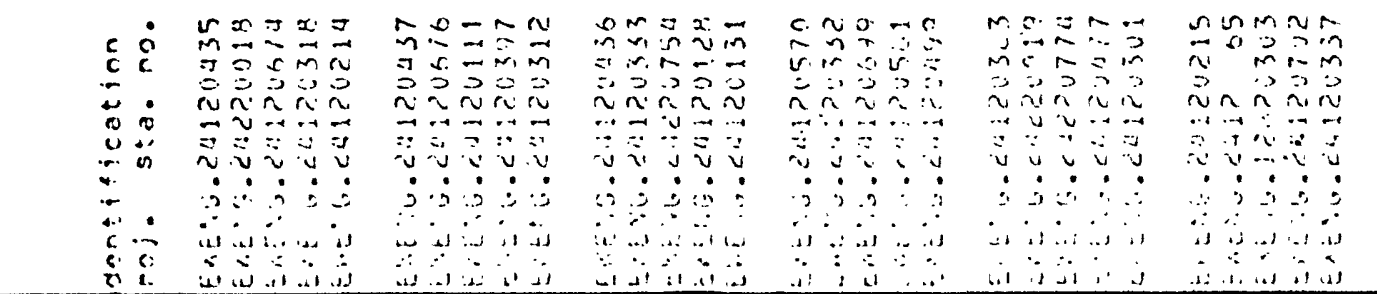

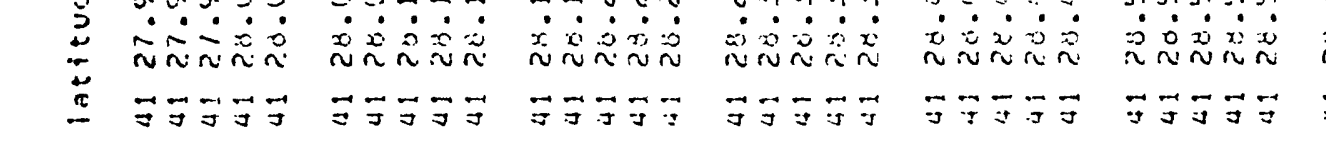
0 $n x x 00$ N

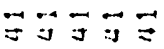

i. 秺宁 unrún $\vec{\jmath} \rightrightarrows \vec{\exists} \vec{\exists}$

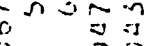
inn? N $\overrightarrow{3} \because \vec{*}$ ป v v $\therefore \div \therefore$

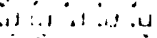

N $\pi N=$ ज. ज्ञ $\therefore=2 \vec{n}$

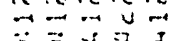
v. v v v $\therefore \therefore \therefore \therefore$ is $\because+1, \cdots$ 
mo5:

$\dot{i} \dot{\sim} \dot{\vec{u}}$

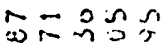

M.: ?

$i \cong \sim \tilde{v}$

ว.บำ

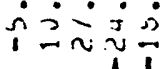

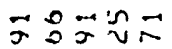

$x=20=$

$\dot{0}=\dot{n} i \frac{1}{1}$

룽요

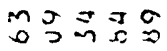

$\dot{0} \dot{\sim} \dot{0} \dot{0}$

83333

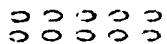

¿0ं்:

$\therefore \dot{0} \dot{0} \dot{0}$

응ㄱ응

30530

0000

年

$n \simeq \Omega 00$ n-nNo

Mํ.

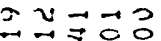
வ0000

ํํำำำ

20303

ச0.

0.07305

$\therefore \therefore \circ 0$

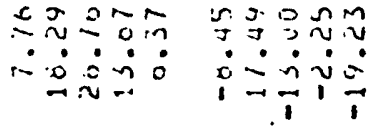

ก 00, in

$\because \because \dot{0}$

$\ddot{\sim} \tilde{\sim} \dot{\sim} \tilde{a}$

T的骂毛?

mลN孚前

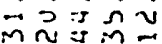

$\therefore \therefore \therefore \div 0$

$=11$

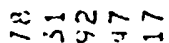

inis

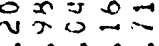

$\therefore \vec{\sim} \dot{\vec{M}} \overrightarrow{\mathrm{m}}$

30030

ว.․․․․․

용ㅇㅇㅇㅇ

வ0ல்

3303

c00 00

$=\because v \hat{0}$ D0ं0ं 0050.

$00=02$ $\therefore \therefore \dot{0}$

\section{m} $\therefore 0^{\circ} 0^{\circ}$

$\vec{\nabla}-\overrightarrow{0}$ वंध

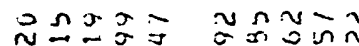

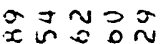

$\vec{m}=$ 政?

조롤

ำกำ

ำ 25

$\ddot{i} \underset{i}{i} \dot{v}$

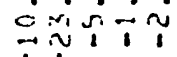

군

11

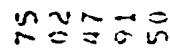

고교

$0-10$

ㅋำณ幺

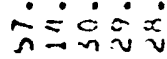

$x \dot{0} \dot{x} \dot{x}$

$\dot{m} \dot{0} \dot{0}$

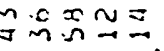

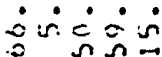

เง $\sim ?$

$\approx n \approx=$

$\hat{\sim} \dot{\sim} \dot{\sim} \dot{\sim}$

$\because \div \div \div \overline{1}$

$00 n n m$

$=\sim \div x c$

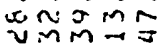

$\therefore ニ ニ \cong ュ$

$0=0 \div$

$x \approx 2$.

$c \dot{s}=\bar{i}$

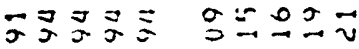

$\vec{\exists} \vec{v} \vec{v} \vec{n}$

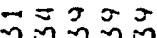

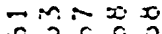

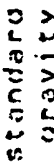

运立定

$\sim \dot{v} \dot{v} v$

$0=0$

nnn.

$\equiv=0=$

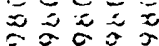

$\dot{\sim} \dot{\sim} \dot{\sim} \dot{\sim}$

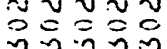

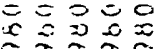

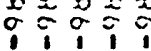

i i i

i $\begin{aligned} & 0 \\ & 1\end{aligned}$

$m a x m$

C.

3
0
2
2
0

$3 ? \cdots \div$

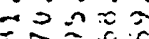

ปv.ง

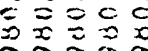

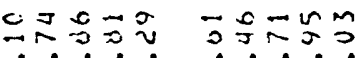

: $\dot{x}$ i

$\dot{v} v \dot{v} \tilde{v}$

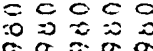

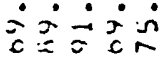

v v

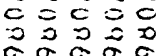

$\dot{v} \dot{v} \dot{v}$

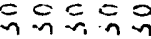

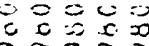

i

$\dot{0} \dot{0}$

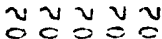

으ㄴㅡㅔ

c

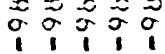

oñN

$\approx x 0=0$ in

$0 \sigma y>0$

웅요 0

政证

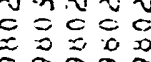

$\approx \approx=n ?$

.200

$\sim$ ข $ข$ ข

000

CMNMM

ONR 0

$\dot{v} v \dot{v} v$

00000

c $0=00$

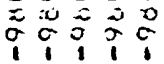

ก $\approx \cong$

$\underline{\sim} \sim \overrightarrow{0} \therefore v$

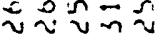

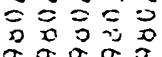

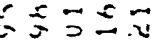

$\dot{v} \dot{n} \dot{n}$

든등

$=0=00$

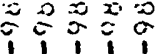

$\stackrel{+}{n}$

-505

๒ேேここ

ㄴㄴㄴ

E $\simeq \cong 2 n$

$02 \pi-2$

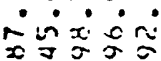

$\dot{x} \dot{0} \dot{x} \dot{0} \dot{0}$

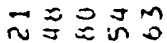

ㄴேㄴ

こั55

กั่ง

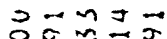

व்ं0்

$\exists \dot{x} \dot{\sim}$

n 0 皮

$\therefore \sim 0 \dot{\sim}$

ํํำ

范灾芯

$\sigma \sigma=00$

$\dot{0}=\dot{i}$

는

$\begin{array}{llll}0 & 0 & 0 & 0 \\ 0 & 0 & 0 & 0 \\ 0 & 0 & 0\end{array}$

픔

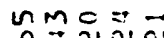

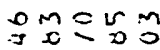

5
0
2
2
0
0

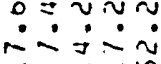

$\tilde{n} \geq \dot{0} \dot{0}$

$\sim \exists \approx \sim ⿻ 上$

in $\dot{0} \dot{\sim} \dot{0}$

$m \sim m \sim=$

$n \sim n=$

นn N

Tmñ

จำ

$\dot{N} \dot{\sim} \dot{\sim}=$

$a m \propto \ln n$

$\approx n \leq \Omega \approx$

$\dot{0} \dot{0} \dot{0} \dot{\sim}$

$m \sim m n N$

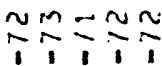

$\div \div \div 1$

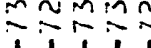

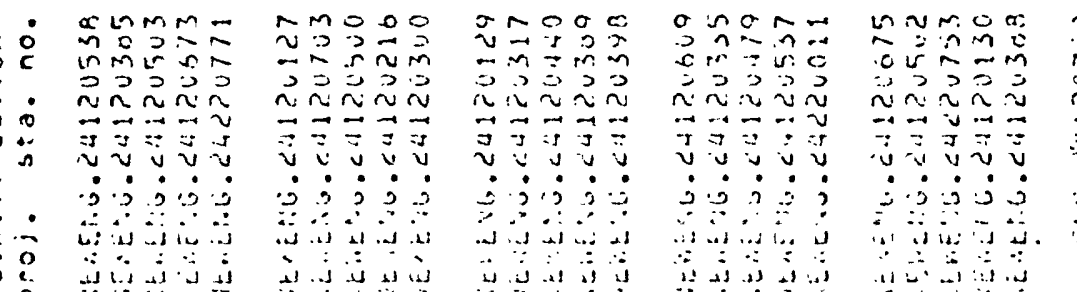

O-No

$\dot{0} \dot{\sim} \dot{0} \dot{0}$

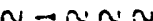

$\pi \div \div \div$
๖ธธง

\section{มก๊}

m $n$

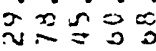

$\because \cdots$

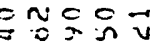

inj $\dot{0}$

$\sim m \sim m n$

$\simeq \pi \simeq \cdots$

$\bar{n} \bar{m}=$

$\dot{m i n} \dot{i}$

들

$\therefore \equiv$

i

$n m-n$

$\approx \sim v$

nก:

然然

응

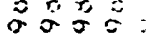

テับ气:

을

$0 \dot{0}=$

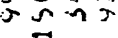

$\Rightarrow \pi=$

$\dot{0} \dot{0} \dot{0}$

जin $=$

-M N

$\div \div-1$

$x 0 \approx \approx ⿻ 上 丨$

NNOO

$\dot{0} \dot{0} \dot{0} \dot{0}$

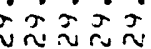

ज可可

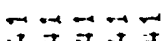
in in यंग $\because 3=\overrightarrow{=}$ $\dot{v} u \dot{v}$ j:30; ני 争 ans

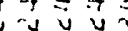

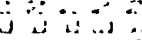
$\rightarrow$ 궁 $\vec{\nabla}=\vec{\nabla}$ $\therefore \dot{3} \dot{3}$

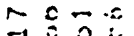

$\therefore$ 的 

$\therefore \dot{0}=\dot{0}$

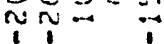

nnn nm

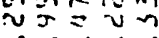

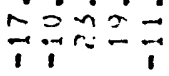

00030

$\because=0=3$

0ं் $\dot{0} \dot{\circ}$

03300

․․․․

$\Rightarrow x \sim L$

$\because\{\approx \exists$

$m o m m=$

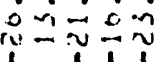

$\because 0 \tilde{\sim}$

$\ddot{\sim}$

m约

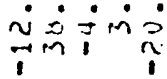

$m \approx \pm \ddot{\tilde{v}}$

$\dot{\sim} \dot{n}=\dot{0}$

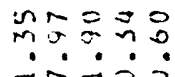

चिते

$\therefore 9000$

공영영

ㄷㅇㄷㅇ

0்

응여잉

- $\dot{0} 0 \dot{0}$

웅여잉 ․․․ வீं

응ㅇㅇㅇ ic 0 :

잉응영 0ं்

ขñ 2

$\vec{\sim} \tilde{\sim} \cong$

$n=50$

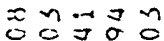
वं: ¿ं0் ¿ं0்

mㄹํ우

nn $=0$ $\dot{0}=\dot{0} \dot{0}$ 0ं்

․ำ ச்ச்

$\equiv 00 m \vec{n}$

낭 .

$\because$

ç

c.

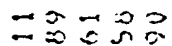

$\Rightarrow 0=00$

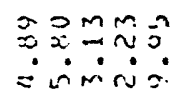

$0 \approx \pi n s$

min

$1 \rightarrow \frac{\pi}{1} 1$

$\therefore \dot{ } \dot{0} i$

$=\frac{m}{1}=1$

ป

$0=\frac{1}{1} \sim$

mंี

$n \dot{=}=\dot{0}$

arisen

$n a=m x$

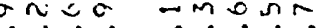

imm

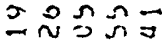
$\therefore \dot{0} \dot{0} \dot{0}$ $\div 1 \div \overline{1}$

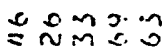

$\dot{0} \dot{0}=\dot{0}=$

$x=0 \pi x$

$\vec{\nabla}=\sigma \sigma$

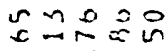

$\dot{a} \dot{a} \dot{0} \dot{c}$

$\dot{\theta} \dot{1} \dot{0}$

$\therefore \therefore \dot{m}=$

กำ

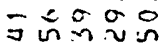

v?

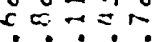

$\dot{v}=\dot{0} \dot{0}$

$=\dot{0} \dot{m}$

$\approx \vec{r} \approx \approx=5$

s. sic $x=$

\section{xan}

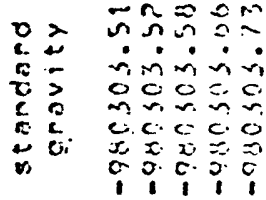

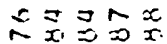

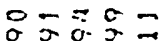

$\exists \approx \exists \approx n$

$\dot{\sim} \dot{\sim} \dot{\sim} \dot{\sim} \dot{r}$

c두의.

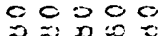

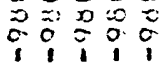

in் $\dot{1} \dot{j}$

등ㅇㅇㅇ

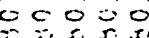

$=0$

उ

C등

no: $=0$

c. c. o n

$\sin 0.72$

osis

vnn

500 $00=0$

$\sim=\dot{0}=$

siv viv

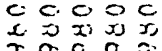

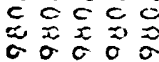

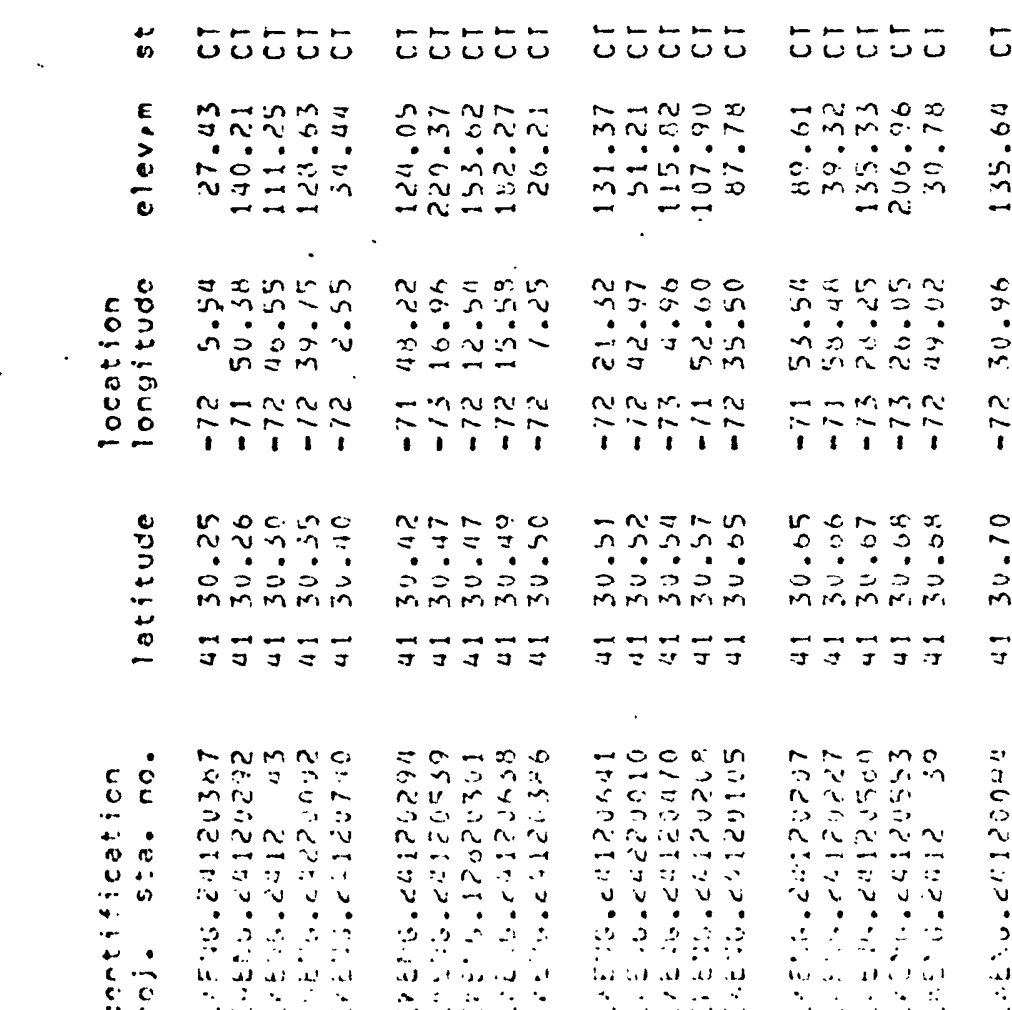

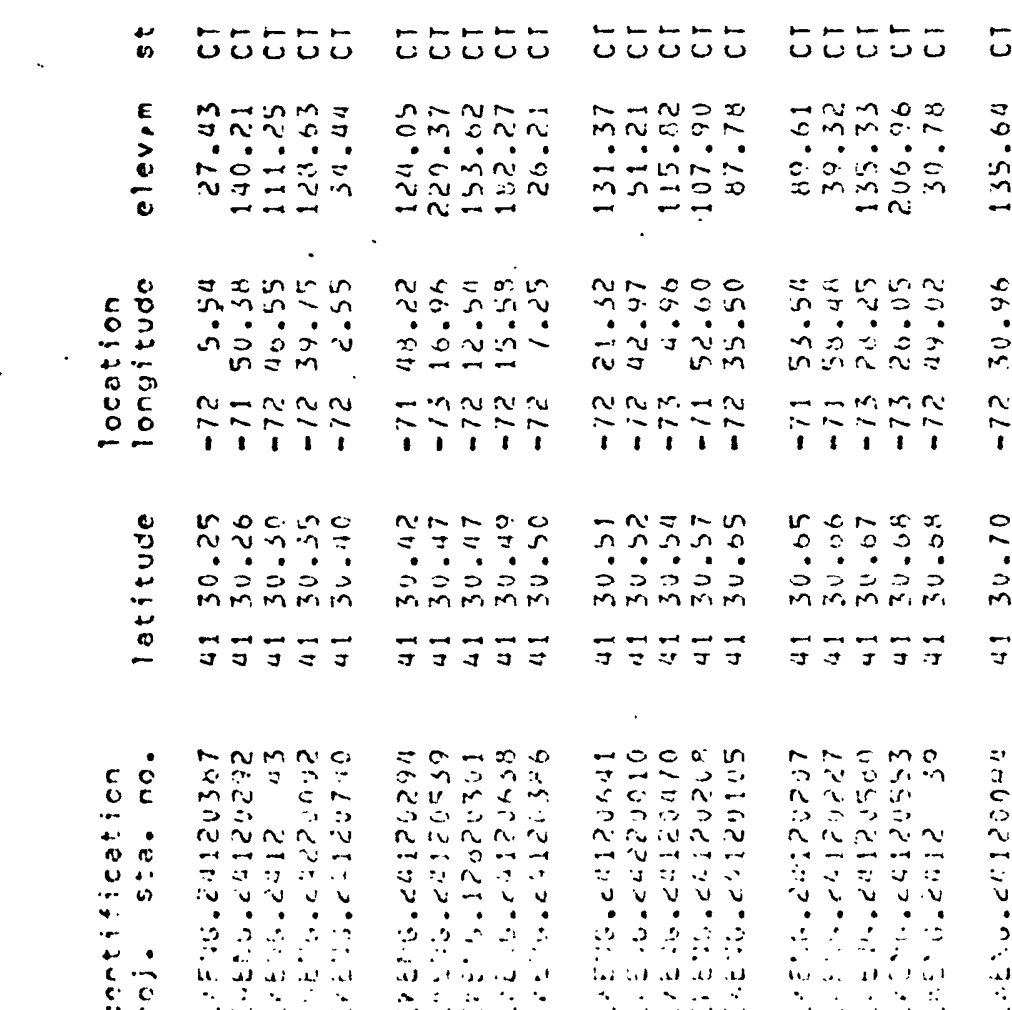

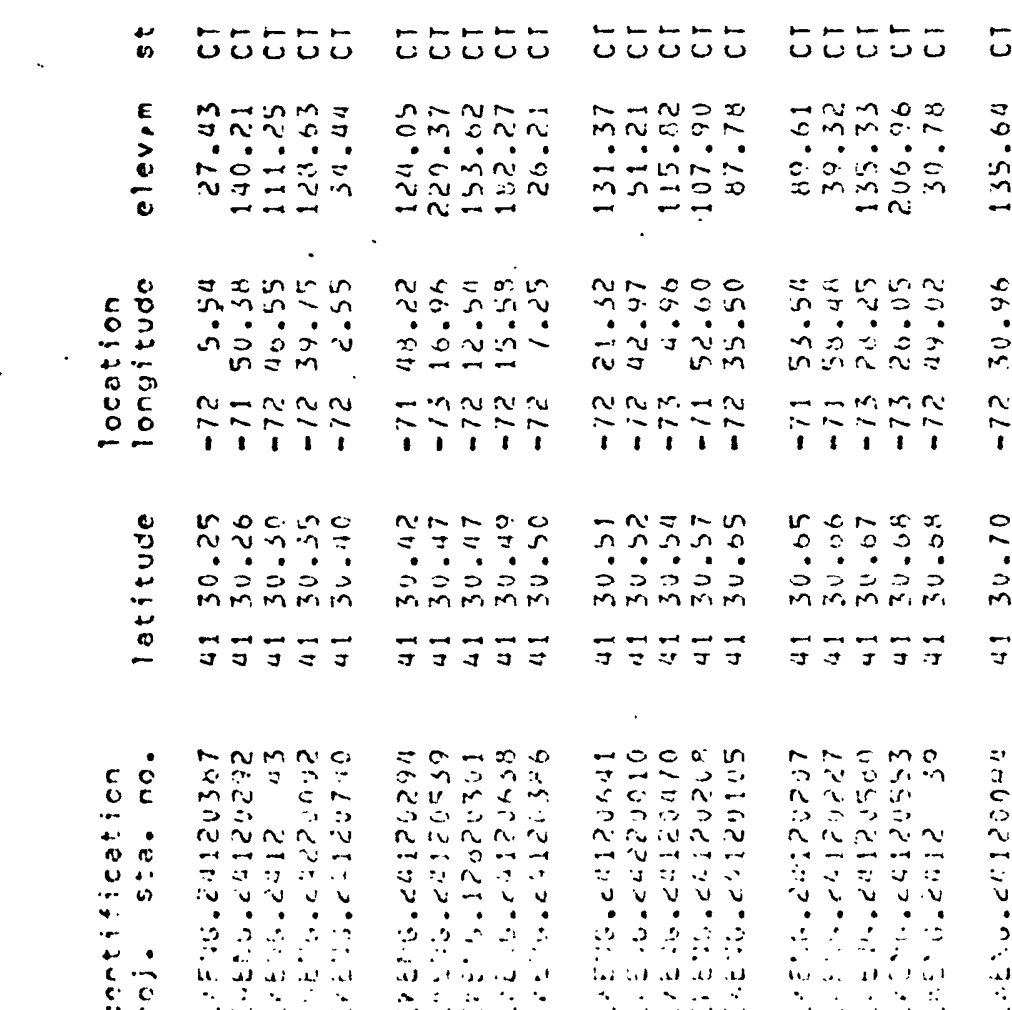

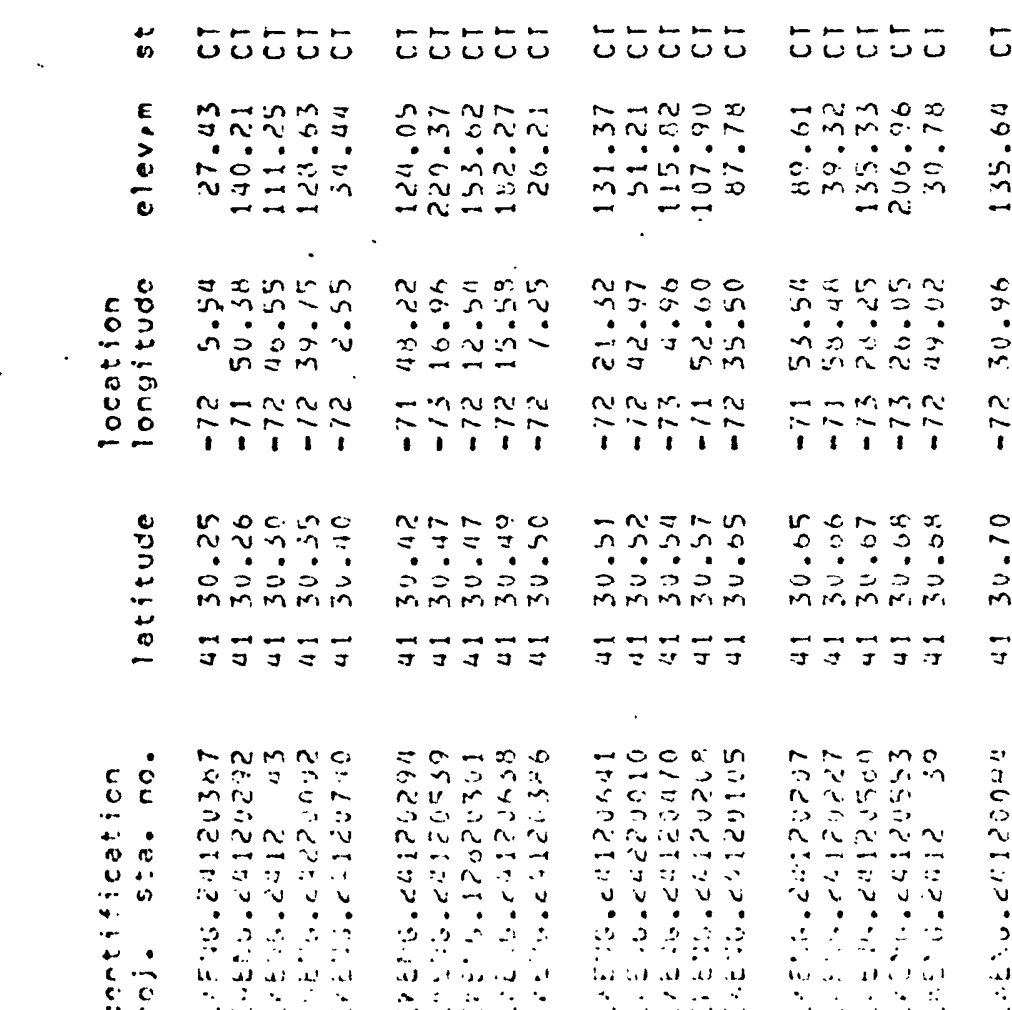

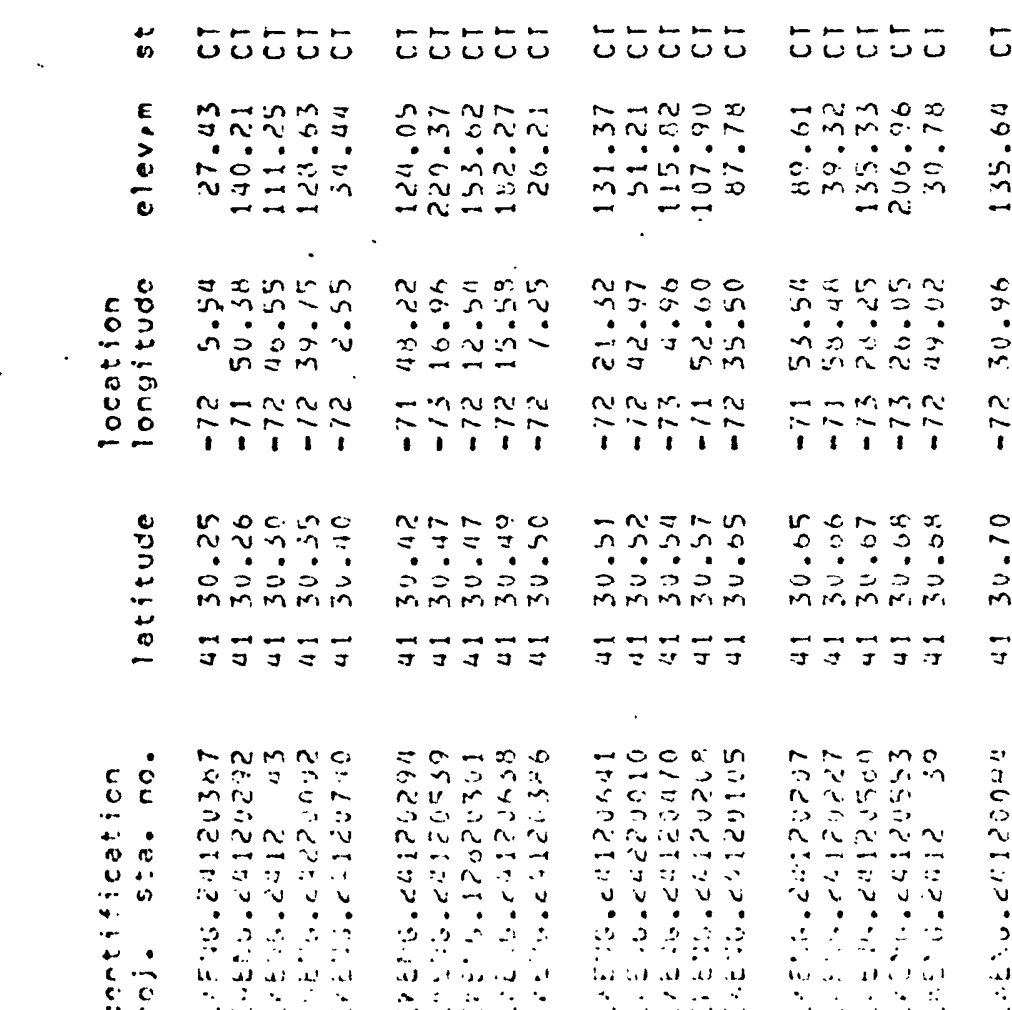

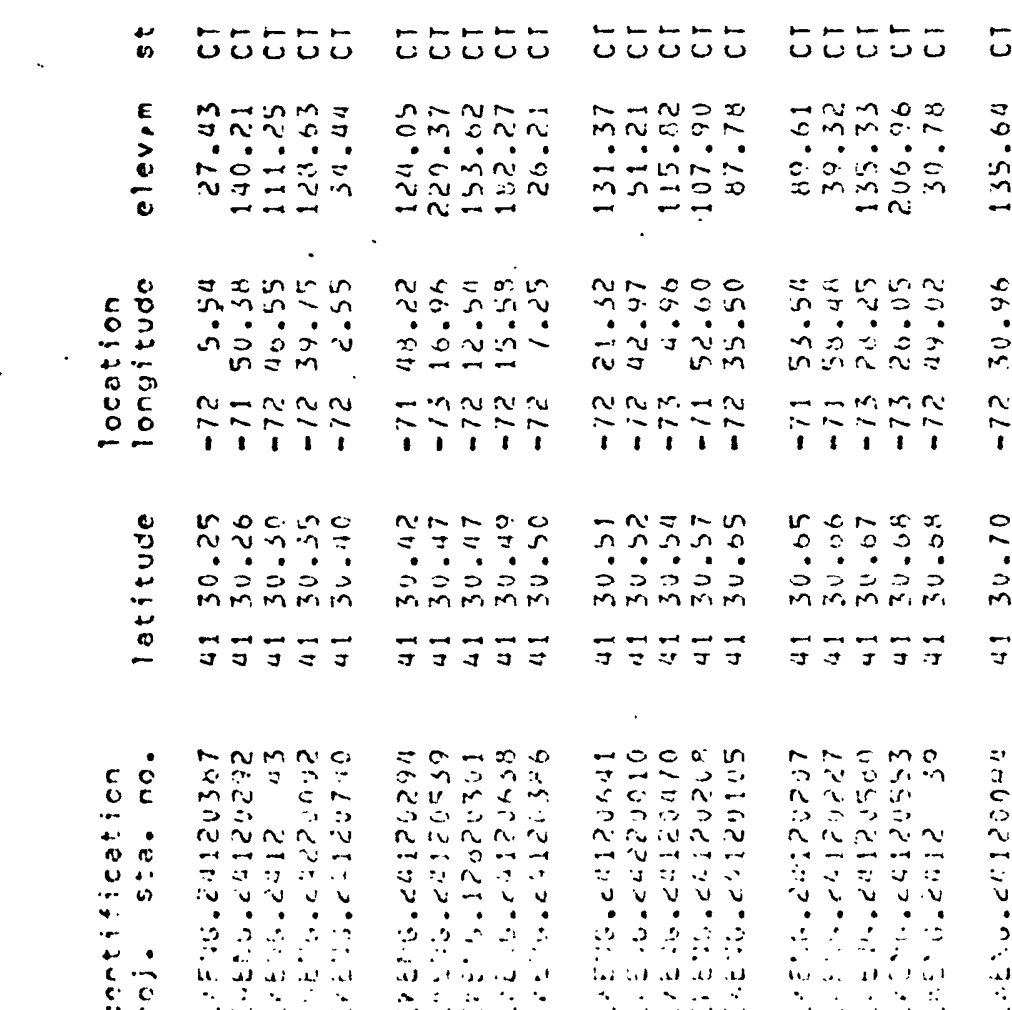

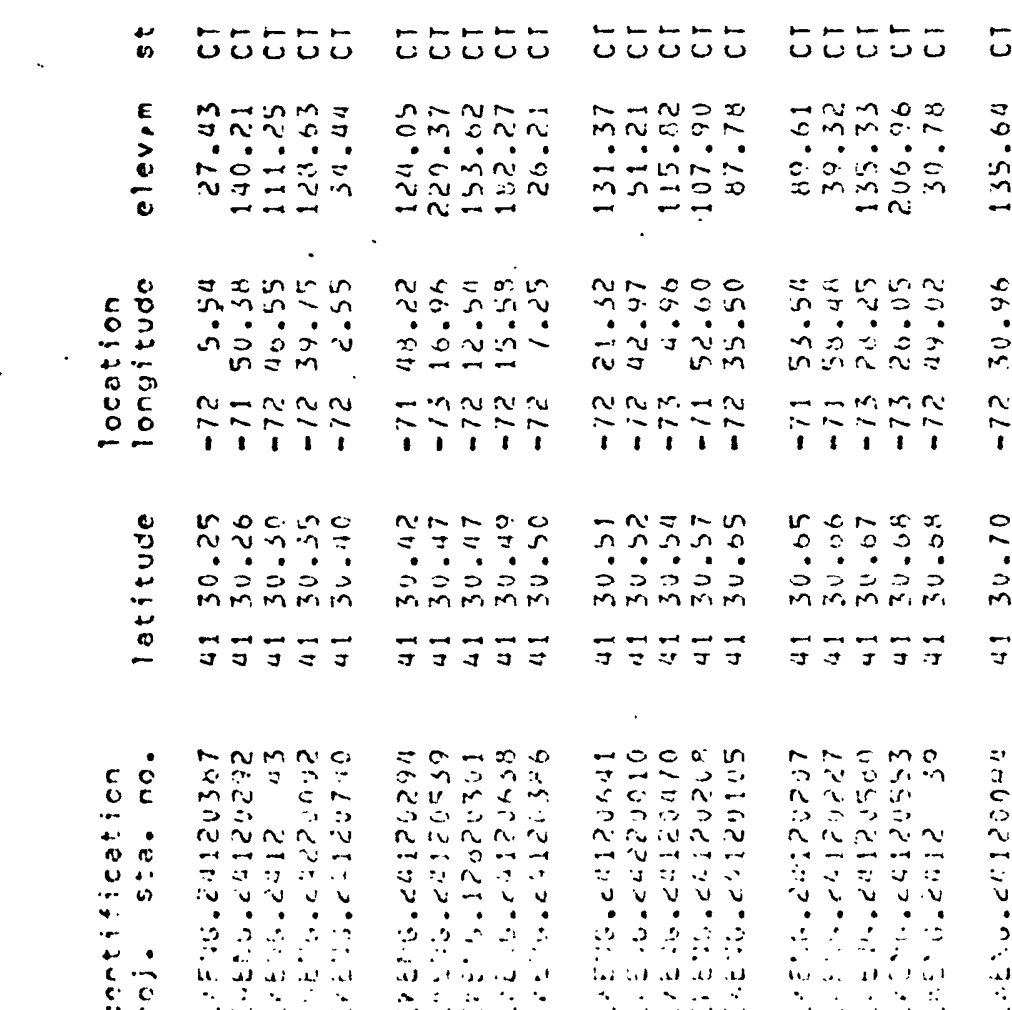

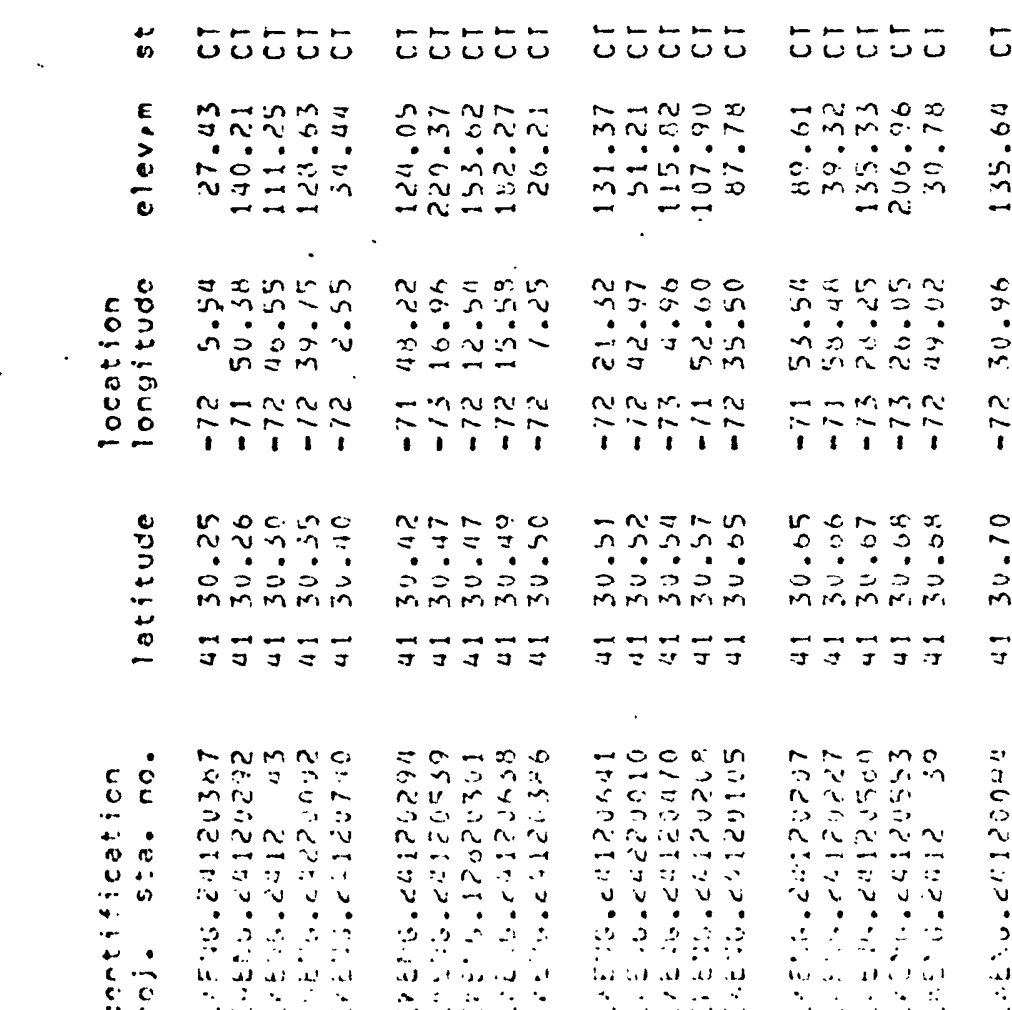

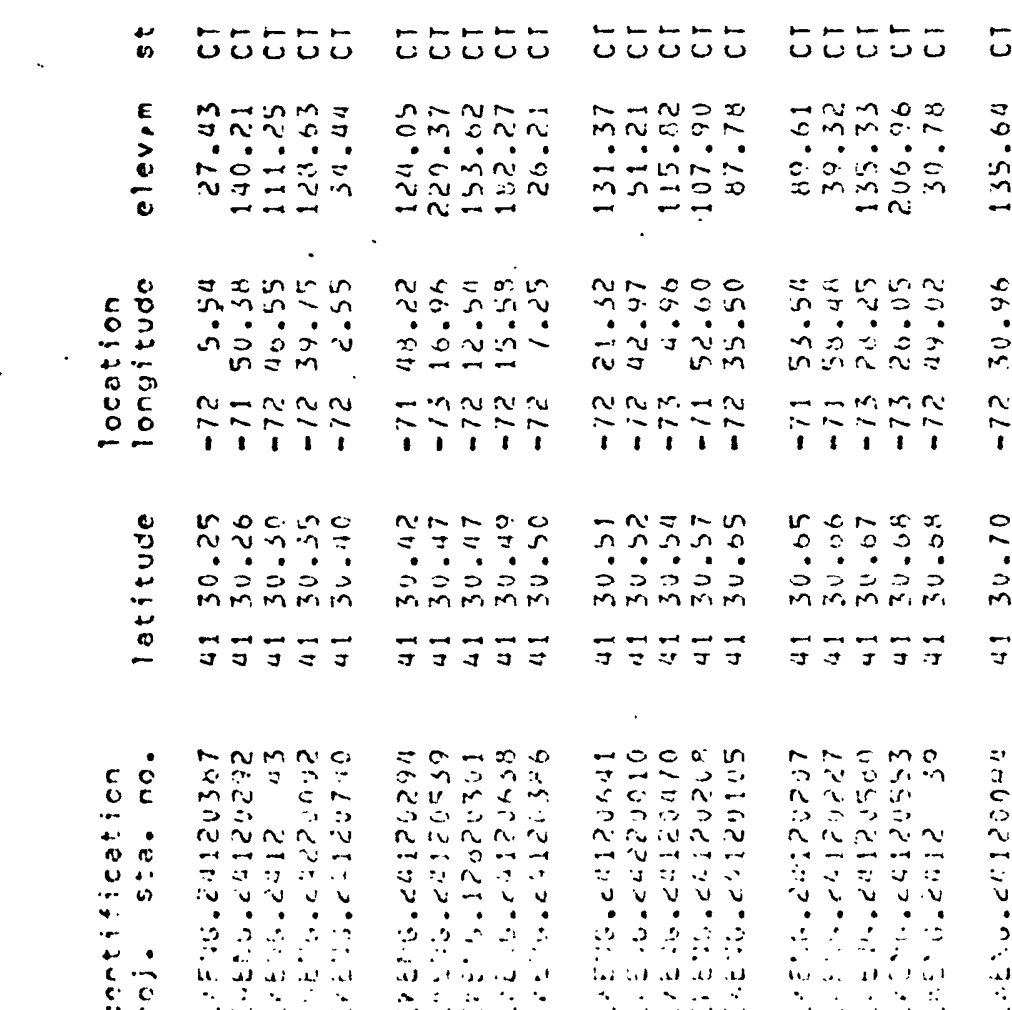

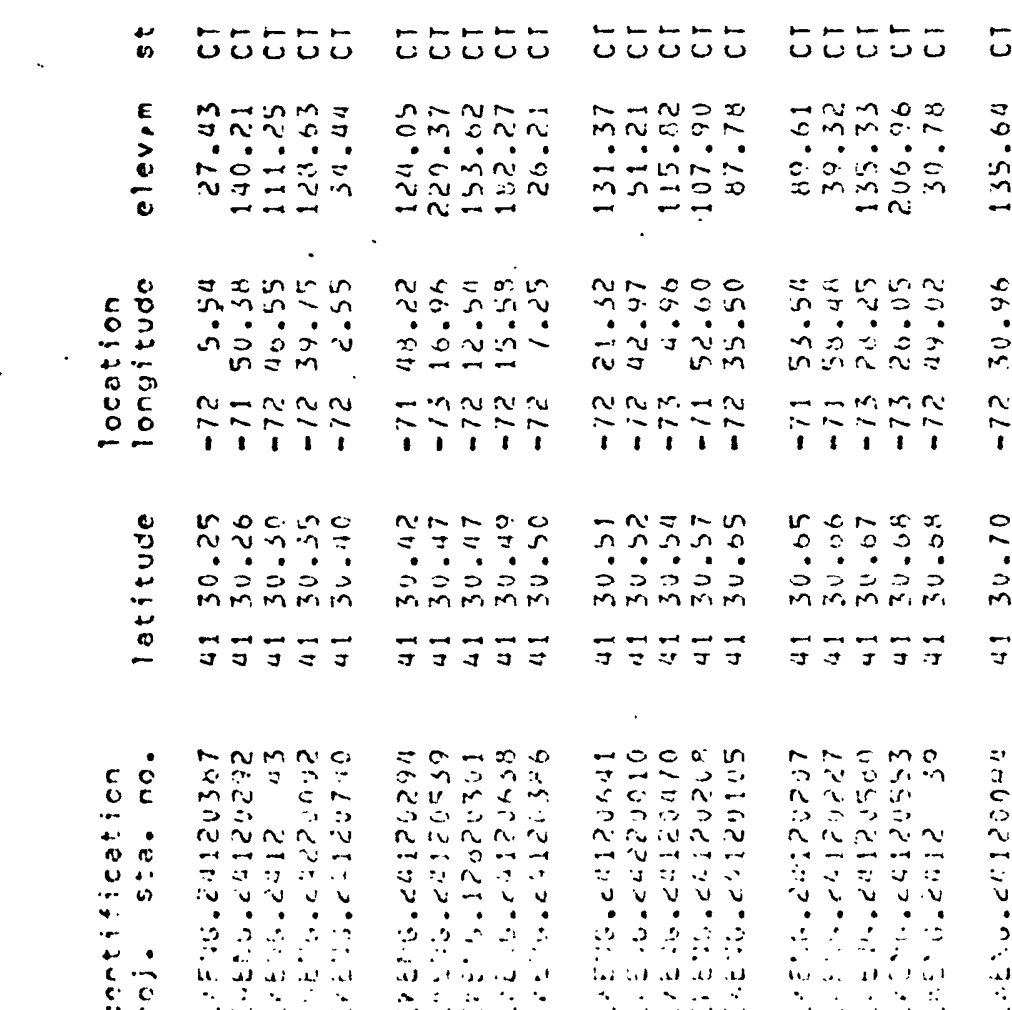

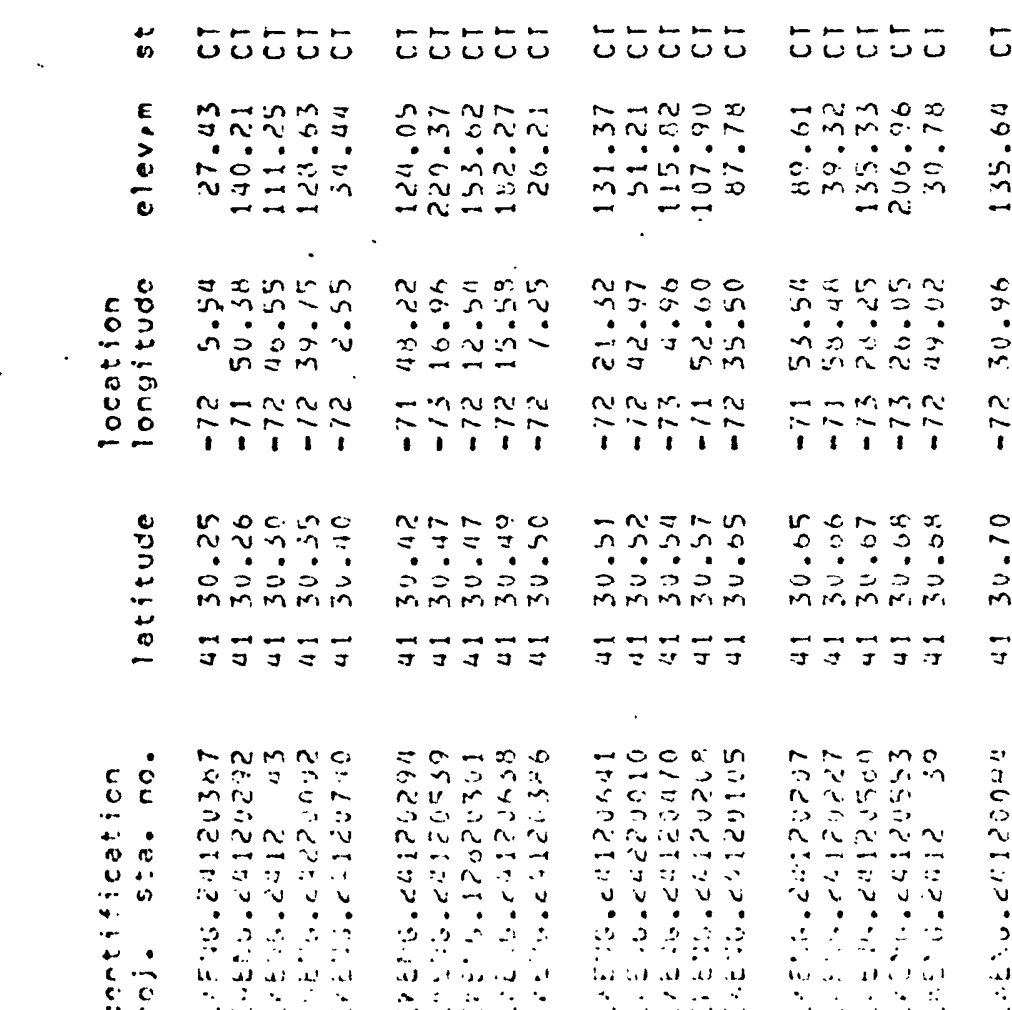

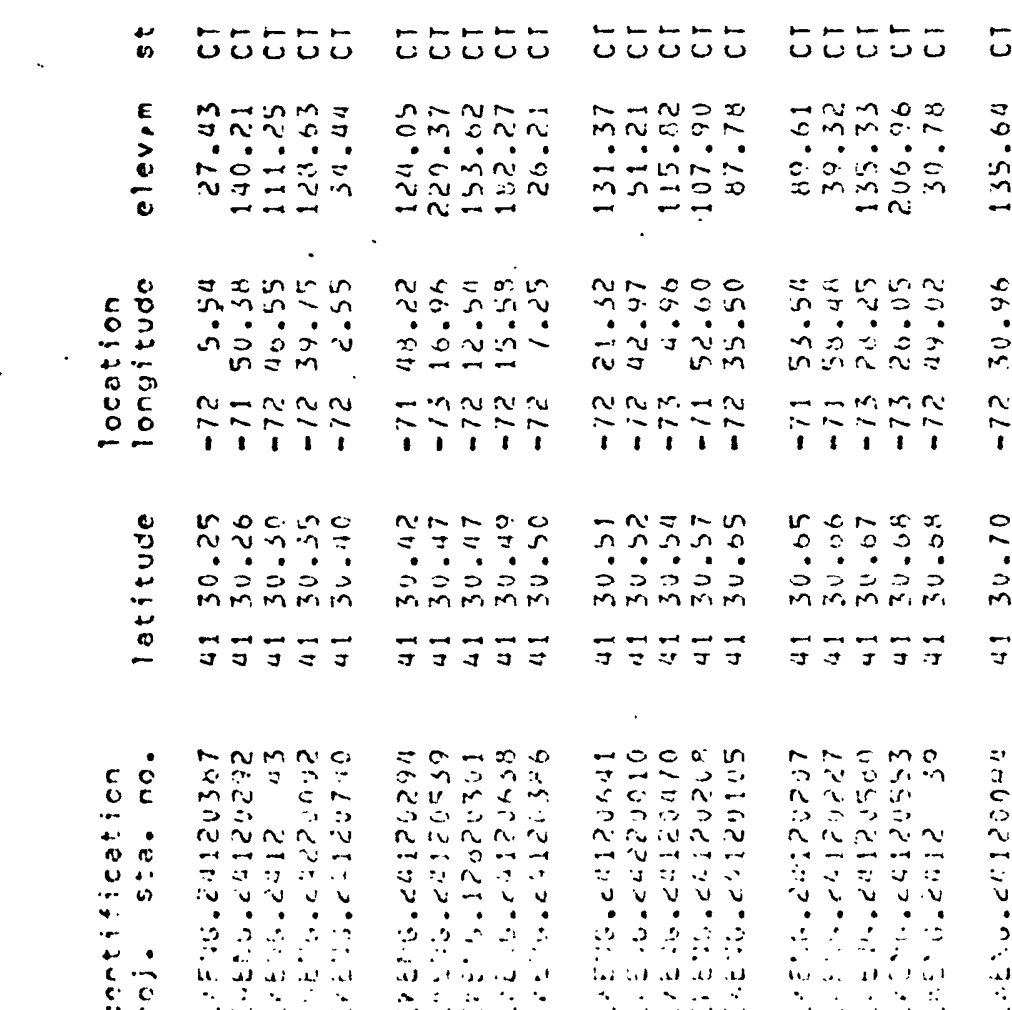

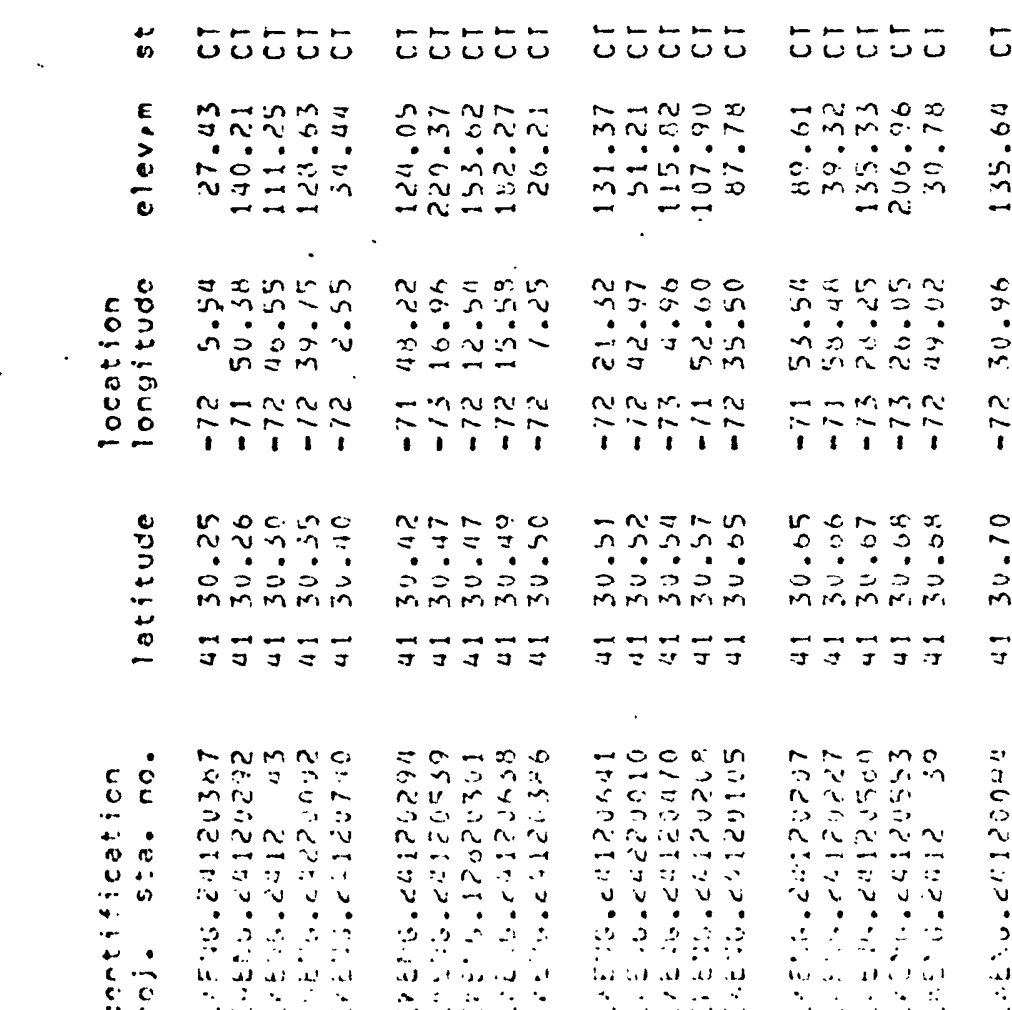

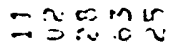

$\therefore \dot{2}=\dot{0}$

v vv

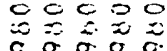

$x$ and

- nuvivi

$\dot{\vec{j}} \dot{\vec{z}}=$

的的n

$\because x=0$

i.

$\sigma x$ in $c m$

-NT. 0 ?

$\sim ⿻ 上 丨$

ขข ข

$x=c 0 c$

m方寻

$\dot{\vec{a}} \dot{=} \dot{\mathbf{E}}$

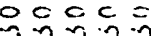

-

$\approx 50$ 웅

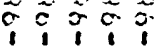

$\overrightarrow{0} \overrightarrow{0}=\overrightarrow{0}$

$\dot{0} \dot{0} \dot{v}$

元记

$\Rightarrow 000=$

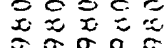

กำ

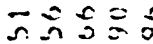

$\dot{\Sigma} \dot{\mathbf{0}}=\dot{0}$

대에

$000=0$

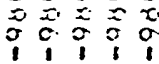

O in m r m

บ: $m$ ?

हैखि

ฟ

00000

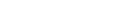

แேேヒே

$=2001$

$=2 m$

$n=心 \cong$

๖๖ぇが

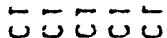

กับร

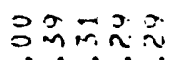

cun $=0$

$x$ in

In n⿺辶一兀

小5口

$\dot{\nabla} \dot{n} \dot{i}$

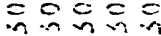

$0=000$

i $15 \%$

$\stackrel{2}{=} \underset{\exists}{ }$

$\dot{a} \approx \approx$

ปv v

$\begin{array}{lll}0 & 0 & 0 \\ 0 & 0 & 0\end{array}$

$-1-$

טúu

m

$\therefore-100$

$\because \approx 00 \pm$

7030

$\dot{0} \dot{0} \dot{\vec{j}}$

r. $x$ in

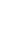

$\approx 50 \ddot{n}$

?

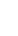

mNom

$-2 \geq \frac{1}{1}$

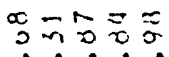
$\dot{\sim} \dot{\sim} \dot{n}=\dot{c}$ nnmin

11\%彳

ON止正

$0=\ln \pi n$

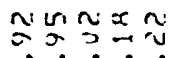

mininino $\dot{0} \dot{j} \dot{j}=$

$\therefore \leq 0 \therefore 0$

किलिए

vก๊

$\ddot{\nabla} \vec{J} \ddot{\jmath}$

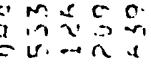

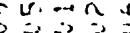
Urúr v v $\therefore \therefore ;$ $3: \therefore \therefore$ 可急宁

लिलिज्ञ テす⿹丁口 $\therefore \rightarrow$ m ज्ञ.

$\vec{\imath} \vec{\sim}=$

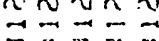

$\exists \ddot{\nabla}=\vec{z}$

iv v v $\therefore 3 \therefore \therefore$ is mac a

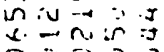

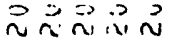

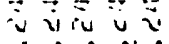
$\because 2 \div \div \div$ $\because$ 品 $\because \ddot{-1}=$ 
Noñ

$0 \div \div \because ?$
$\dot{1} \div \div \div$

o o in

u. x r

$\vec{\sim} \vec{v}=\vec{\sim}$

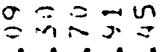

$\therefore=\dot{0} \dot{0}$

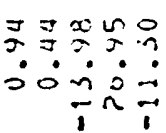

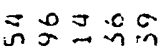

ज计?

i

จำ

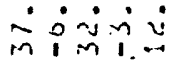

응잉ㅇㅇ?

경영영

○்

ல0் $0 \dot{0}$

2. 3903 -0000

20301

园类品

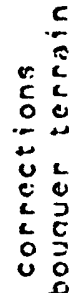
이이

$\therefore \therefore \therefore \circ:$

$\because 3 \approx 0 \mathrm{C}$ $\therefore \dot{0}=\dot{\circ}$

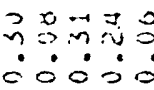

으요을

- $\dot{0} \dot{0}$

Mํํำ ¿0ं0.

$02 \vec{\nabla} \overrightarrow{0}$

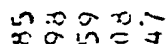

गलिज्ञ

$\dot{c}=\dot{m} \dot{x}$

i. $\dot{0}$

$\because \dddot{\sim}$

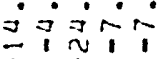

$\overrightarrow{1} 11 \overrightarrow{1}$

$m \leq 00=0$

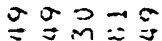

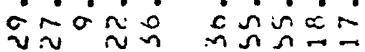

$\therefore$

3
$=$
2
3
3
2
2
2
2
3
0
0
0

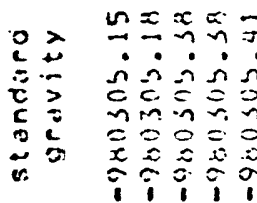

$m \sim ⿻ x$

$\because \Omega ?=0$

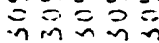

$0=00$

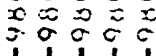

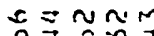

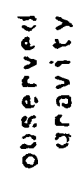

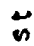

$\stackrel{e}{e}$

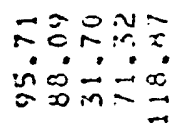

$\begin{array}{ll}c & 0 \\ 0 & 5 \\ 0 & 5 \\ 0 & 5 \\ 3 & 5 \\ 0 & 0 \\ 0 & 0\end{array}$

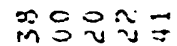

in in $0 \dot{0}$ in

乌

$\dot{-} \rightarrow N \sim=$

$\pi \div \div \div$

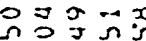

$\dot{0} \dot{i} \dot{i} \dot{j}$

$\sim \vec{x} \geq \hat{n}$

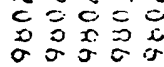

¿ $v \underset{\sim}{x}$

$\begin{array}{llll}0 & 0 & 0 & 0 \\ 0 & 0 & 0 & 0 \\ \sigma & 0 & 0\end{array}$

กำ

$\dot{\sim} \dot{\sim} \dot{\sim} \dot{v}$

$x \circ m=0$

$\because=\approx 2$

$\hat{\imath} \cong 0 \hat{0}$

$\equiv \equiv 00$

$=000$

$=0 x m \sim$

$\dot{\vec{c}} \dot{\vec{x}} \dot{0}$

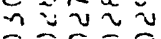

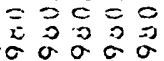

ज屾可

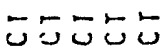

$\approx m \sim 5$

$\because \approx \cdots \sigma 0$

$=0 \sigma 0.0$

$=\sim \infty \sigma$

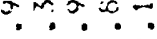

vin $=0$

s.ong ini: $\dot{0}$ $\overrightarrow{1} \overrightarrow{1} \overrightarrow{1}$

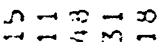

$\therefore \dot{\therefore} \dot{\therefore} \therefore$

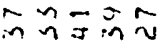

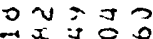
mi $\dot{0} \dot{m}$

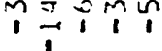

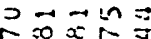
$\therefore \dot{x} \dot{0}$

กำ:

$\because M \approx \sim \sim \dot{x}$

1113

ลก กำ

กำ?

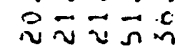

mainsin

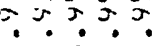

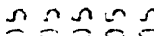

in $\bar{n}$ 的

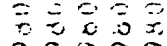

$\because 5050$

$x \approx n m c$

$\because \div \div \div$

ก $2 \leftleftarrows 0$

in vinn

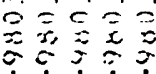

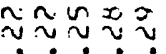
02030 in nin - $=0=$ 20000

on $x$ in inc

$\therefore \dot{0} \dot{0}=$

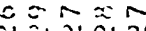

$\begin{array}{lllll} & & & \\ 0 & 0 & 0 & 0 \\ 0 & 0 & 0 & 0 \\ 0 & 0 & 0 & 0 & 0\end{array}$

11111

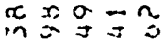

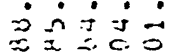

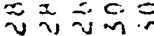

$\begin{array}{lll}0 & 0 & 0 \\ 3: 0 & 5 & 0 \\ 0 & 0 & 0\end{array}$

o $\sim m o$

$\therefore \dot{0} \dot{0} \dot{x}$

$\sim v v v v$

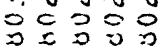

ธٓர口

$5-5-5$

$\because \approx v \div$

- $\div=$

ㅎํำ

$\exists 0 M \approx=$

D迹定

$\sigma \approx x$ in

$\therefore \circ$

ก๊กข

$0.000=$

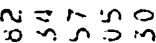

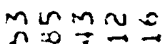

$\because \dot{0} \dot{i} \dot{n}$

moOn

คำ

分证?

NMn-N

m $\rightarrow$ in $\rightarrow n$

$\because \div \div \%$

$\because \div \frac{1}{1}$

ב3

Nㅡㅇㅢ $=0$

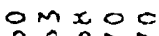

os onin

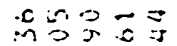

D $=\dot{m}$ min

$\therefore \dot{\sim} \dot{0} \dot{0}$

N几n几-

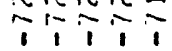

$N m a n$

$\div \div \div 1$

$\approx \sigma \approx \approx 0$ $\vec{\sim} \rightarrow \dot{0} \sim \dot{n}$

$m n-m n$

$\div \div \frac{1}{1}$

$r \approx x x$

$2 N 00 N$

- .

.

$\because ? ?$ ?

$0=0 x$

$\ddot{n} \ddot{m} \ddot{m} \ddot{m}$

$2 \pi>5.5$

min viv

$00 x 0-$

M

ivinis

$\rightarrow \vec{G} \vec{\square}$

$\vec{J} \vec{J} \vec{J}=$

$\vec{\exists} \vec{\nabla} \vec{T} \vec{J}$

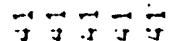

$\vec{\jmath} \vec{J} \vec{ज} \vec{J}$

$\vec{\exists} \vec{J} \ddot{\exists} \ddot{~}$

ตำ in: 2 in

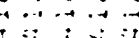
is v u u ; ;

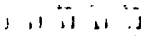


$\begin{array}{ll}\dot{i}=0 & 20\end{array}$

cr

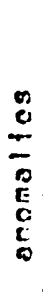

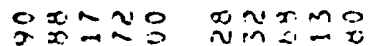 \\ $\therefore \dot{0} \dot{0} \dot{0}$ \\ $=120$ \\ ív $\dot{0} \dot{0}$ \\ $u_{i} \geq \vec{n}$ \\ $m 5 \exists w \omega$

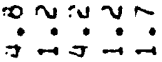

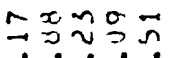 \\ $\dot{i} \dot{i} 0 \dot{0}$}

잉으응

둥ㅇㅇ

30500

$\dot{0} \dot{0} \dot{0}=$

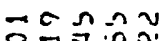

$0 \% \exists$ 约

กำ 20

$\therefore \dot{0} \dot{0}$

$\therefore \circ 050$

con in in

$\because \div$ c i in

1\%?

$x=m \leq 0$

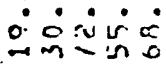

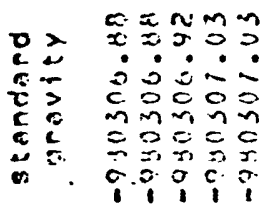

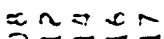

$\because \because \div \div \div$

$\cong 0005$

co:둔

$0=500$

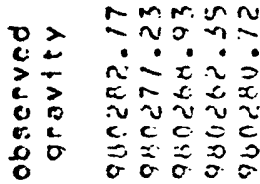

$0-\pi=$

$\therefore \dot{0} \dot{0}$

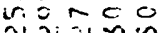

$\approx v-v$ n

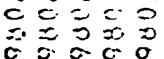

* ㄴபㄴ

$E \quad \infty \infty 00$

\&

ここテこ5

$n n=20$

$0 x=c 2$

mes

:

cuntur ㅇำ? 我讨定

$\bar{E} \cong \sim$ $\div \div \div \hat{1}$

三c品 $\therefore \dot{i} \dot{0} \dot{0}$ $\therefore m=0$ NNONM 1111

ninsu $\because v \cup n m$ की Oc드요 오을 $>\approx 00$

ocruar $\therefore \therefore i \div$

30303 $\dot{0} \dot{0} \dot{0}$

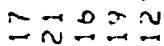
¿

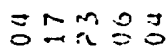

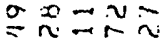
¿व:0:

$\vec{m} \approx \vec{n}=m$ $\therefore x+\frac{1}{1} \div$

00제 in:ai

$\vec{\sigma} \approx m \overrightarrow{0}=$

$\vec{\nabla} \vec{\nabla} \approx$ - ori.

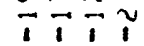

o $x \propto \dot{m}$ $1 \div$

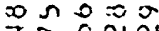

$\therefore \therefore m=$

con

iñن

$\exists \sim \tilde{i}$

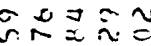

$\dot{n} \dot{0} \dot{0}$

$\sigma N \equiv m v$

$207=0$

$\vec{x}=\vec{\sigma}=$

กิำ

- M- v

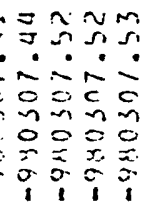

รก⿻上丨丶

$\because 00=2$

$\hat{c} \hat{c} \hat{c}=\hat{=}$

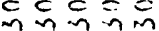

$c c=c$

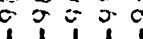

o stown

$\dot{v}=\dot{e} \dot{i}$

o inn

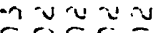

000.

$\propto 0 \sim 50$

$\approx 00 \Omega$

$\therefore \dot{0}$

ข $\approx=\approx$

c c = c c

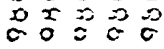

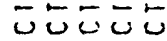

วחㅇำ

ำㄴำ

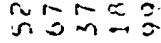

テこピヒ

믄

- 0

กร๊ำ.

$\therefore \therefore \dot{0} \dot{0}$

순

rv记

o 000

in் $\dot{0}$

ปึก

$\dot{\sigma} \dot{0} \dot{0} \dot{\infty}$

$\Rightarrow$ ำำำ

$\therefore \dot{0} \dot{0}$

$x=5=\pi$

is $5 x$ ?

$0=x x=$

$\cdots \cdots$

으으ำ

orooc

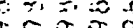

$15 \%$

ข $\simeq \geq 50$

$\therefore<\dot{0}=$

$v v v-$

$\begin{array}{llll}0 & 0 & 0 & 0 \\ 20 & 0 & 0 & 0 \\ 0 & 0 & 0 & 0\end{array}$

ก. $\sin 2 \pi$

$\approx \approx \sigma 0=$

$\sim \sim \hat{0}=$

nn $v_{1} n$

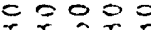

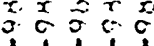

ㄸำ 0 $\therefore \dot{0} \dot{0} 0$ nn o이요 or 050

$=5 \approx N N$ 向完家:

vง v

c 000

c50

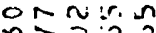
กะว: 은 $=20$ ㄴ. $0 \begin{array}{ccc}0 & c & c \\ 0 & 0 & 0\end{array}$ 0000

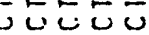

-20-

뚠ㄷㄴ

$\approx 0 \approx \approx 0$

Bम̈-

o ommo

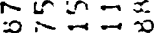

$\tilde{0} \approx \hat{0} \approx$

$\vec{m} \vec{m} \vec{\sim} x$

? $m 5 x$

$\therefore \sim \sim ⿻$

D I n 0

$\vec{x} \tilde{n} \sim \mathcal{n}$ 요의

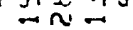

ronir

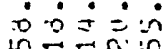

$\dot{0} \dot{0} \dot{0}$

$\vec{\nabla}=0.0$

ก๊ $\approx \cong$

-orntn

$\approx \sim ⿻ \overline{7}$

$\therefore \sim \dot{\sim} \dot{0} \dot{0}$

นn

ninis-

- $m$ u $u$

$\therefore \dot{0} 0 \dot{0}$

$\therefore \sim 0 \dot{0} 0$

$m \sim n \sim n_{1}$

mann

1111

11\%

$1 \div 11$

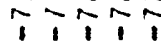

moruma

$11 \% 1 \%$

แี่

NNAN

11;

oomoc

$=n x 00$

min $r . n=$

c $x m n_{i}=$

$x, 0=0$

ner on

$\because \because 20$.

$\cdots=5=$

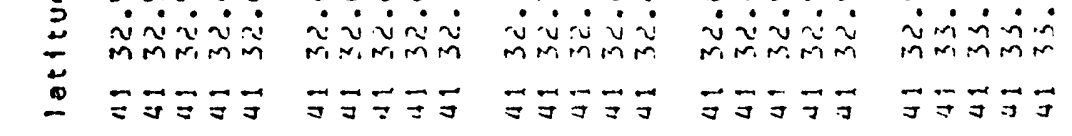

nn n

on $N=0$

บง

innin

mmimm

n m n in o

$\because \vec{\jmath} \vec{\jmath}$

将艼可

$m n m n \cdot m$

$\vec{G} \bar{\Xi} \bar{\Xi}$
요의 w5s $n$ in

…남

$\because y+\exists=$

v.v v v v

$\therefore \therefore \therefore$ :

is $x<\sigma=m$

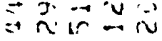

$\ddot{2} \vec{n}=$

$\because \approx \ddot{\sim} \because \ddot{\square}$

v. v v

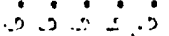

is 4 .

4 is 
$\because \because \cdots \div \div$ บ?

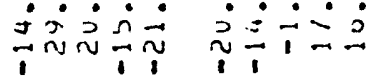

$\therefore$

2.

至

: i

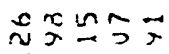

$\ddot{i n} \approx \dot{\sim}$

$m=0 \%$

$\dot{m} \dot{0} \dot{0} \dot{0}$

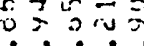

$\therefore \dot{\sim} \dot{\sim} \dot{\sim} \dot{1}$

$\dot{0} \dot{i} \dot{i} \dot{i n}$

$1 \frac{1}{1}$

응영영

03933

0ல0ல0

$\dot{0} \dot{0} \dot{0} \dot{0}$

$\therefore 3930$

$\therefore \therefore \dot{0} 0$

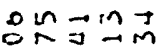

$\dot{0}=\dot{0}=\dot{0}$

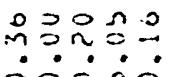

무을

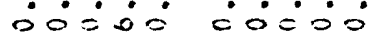

c

-

c

¿.

ㅇํㅇ

$\because \stackrel{2}{*} \simeq \stackrel{0}{0}$

i

$\div$

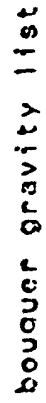

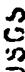

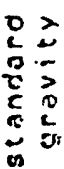
$-\sim_{1} \rightarrow$

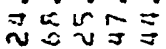

ㄷำ $=0 x$ $x x \dot{0} 0$

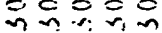

cocico

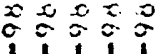

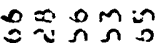

2.

$\dot{x} \dot{\nabla} \dot{v} \dot{0}$

v $\bar{n} \tilde{v}$

$\begin{array}{lll}200 & 0 \\ 000 & 0 & 0 \\ 0 & 0 & 0\end{array}$

$\ddot{0}$

ธこちこう

$E n 0 x=0$

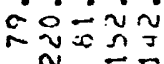

$\tilde{x}=\Omega^{2}=$ $\therefore \therefore \therefore \dot{0}$

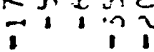

$\because 00 \hat{n}$ $\therefore \dot{c} \dot{c} \dot{x}$

$11=$

$\tilde{n} \approx-\sim \vec{n}$

$\dot{0} \dot{\sim} \dot{0} \dot{0}$

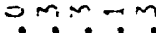

든

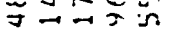

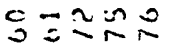
$\because \div \div$ $\approx$ D. D : 0 듯 $0=5 \%$

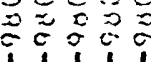

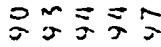
$x=00$ 도요용 $0 \equiv$

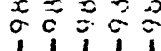

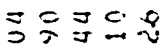

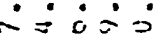

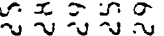

$c=0$

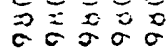

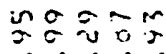

$\therefore=2-1$

元

c c $c 00$

完市完市

$5=5-$

$5-5=-$

orsug

$-x x=m$

$\ddot{*}=\ddot{m}$

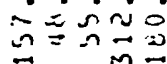

$\approx m$

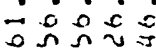

ดก

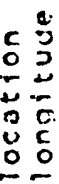

กำ

in $\dot{\sim} \dot{\sim} \dot{0}=$

$=\pi m \sim n$

$\div \div \div \%$

ก ก $\because ? ? \div \div \div ?$

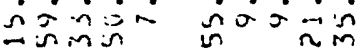

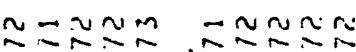

$1 \div \div \div$

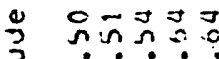
空

$2 \dot{x}$

$\Rightarrow x$

ơ

:

$0 \underset{5}{2}$

$\equiv \ddot{x} \ddot{x}$

西

20

is

In omno $n \pi \infty$

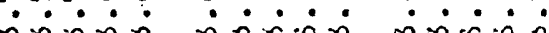

mimmin

miñ in mim

$\vec{\jmath} \vec{\jmath} \ddot{\Xi}$

$\vec{\exists} \vec{J} \vec{J} \vec{J}$

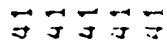

$M \because=\dot{G}$

$1 \div$

s. 200

$x 0 x>0$ iñ

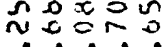

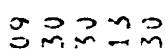

n $n \geq 0$

$\ddot{i} \dot{0} \dot{0} \dot{0}$

$\vec{i} \dddot{i}$

nก 0 m

0.00

$\dot{i} \dot{i} \frac{\dot{0}}{1}$

ㄷำ

$\dot{\overrightarrow{1}} \dot{\square} \dot{\overline{1}}$

$\because 05 \overrightarrow{0}$

$\ddot{v} \div \dot{i} \div$

30003

$\therefore 3>0=$

23000

ப் $\dot{0} \dot{0}$

$\therefore \circ$.

$=0000$

$\therefore \dot{0} \dot{0}$

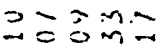

வல்0்

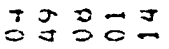

$\hat{0}=\underline{M}=$

¿0்

$\sin \pi$

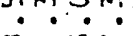

$\overrightarrow{1} \overrightarrow{1} \div \overrightarrow{1}$

공ำ

$\dot{0} \dot{0} \dot{\sim} \dot{0}$

的的

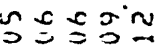

?

류워

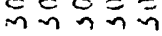

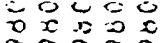

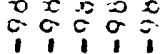

요요

$\therefore \dot{0} \dot{j} \dot{J}$

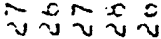

$\begin{array}{llll} & 0 \\ 0 & 0 & 0 & 0 \\ 0 & 0 & 0 & 0 \\ 0 & 0 & 0 & 0\end{array}$

$\sim \simeq \simeq \Sigma \tilde{v}$

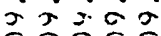
$m \sim n n=$

$=\div 0=0$

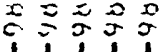

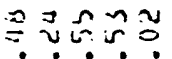

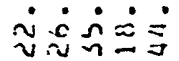

$\cong \stackrel{v}{c} \bar{v}$

$\therefore \dot{0} \dot{0}-$

vำ

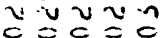

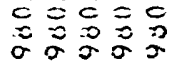

可汹

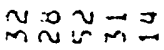

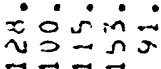

5555

ก롱

$\because \sim n c \dot{0}$

B.0.0n

วิ요

กำำ

둥 050

$\dot{0} \dot{i} \dot{0}$

$\sim \sim N m-$

ำN

11,1

mंलिभिज $n=0 x c$

今00\%0 inini 可牙可

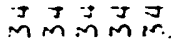

$\because \vec{J} \vec{J} \vec{J}$
굴

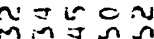

.

$\alpha \pi \approx \sigma a$

ñn $n$ n

$\equiv \equiv$

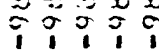

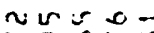

noming

$\sim \dot{0} \dot{0}=0$

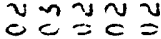

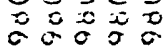

ேேーレ

$20 \overrightarrow{2} n$

$\therefore \dot{0} \dot{0}$

$\begin{array}{lll}x & x & 0 \\ 0 & 0 & 0\end{array}$

○n-ñ

ann $n=$

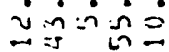

nn-n

$\div \div \div \frac{1}{1}$

$m$ in $\sim$ ก

$\because \because \dot{0}$

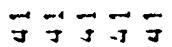

ํำำ

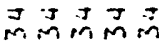

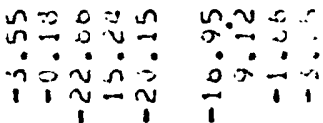

$\cong \approx \approx ⿻ 上$

$\Omega \mathscr{s}=$

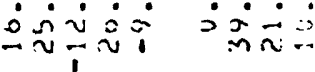

$02=32 \quad 033$ - $\dot{0} \dot{0} \dot{0} \dot{0}$

จง 00000000

$0=2 \pi \sqrt{2}=$

$\dot{\nabla} \dot{0} \dot{0}$

i $1 \overrightarrow{1}, \overrightarrow{1}$

ก̂.

$\therefore$ ¿nim

$\overrightarrow{1} \tilde{1} \overrightarrow{1}$

$\stackrel{n}{=} \vec{x}=$

$0 m=0$

$\therefore \dot{0} \dot{0}$

$\dot{x} \dot{m}$

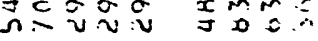

$\exists N=F$

in 2002

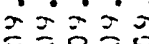

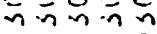

0500

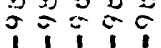

$\sin \pi x$

$\dot{0} \dot{0} \dot{0} \dot{0}$

viviv

c c $\begin{array}{ccc}0 & 0 & 0\end{array}$

वर्व 000

$x=2$

$\therefore \dot{0} \div$

$0=$

nin $=$

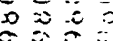

11

บ $\tilde{\Omega}=7$

$\dot{v} \dot{i}-\cdots$

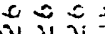

$000=$

는

$-5$

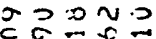

กิก

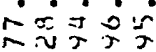

$\because N$

m궈요 


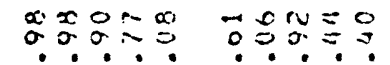 \\ isis \\ $\approx \frac{\pi}{1} \div 1=$

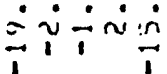

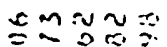

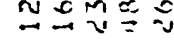 \\ $\vec{i} \dot{i} \dot{i} \dot{0}$ \\ İis}

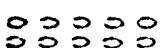

?.?

○்வல்

80300

․․․․․

$\therefore \dot{0} \dot{0} \dot{\circ}$

$m x n m m$

$\because 0 \div-7$

00ं00

ㅇํ융요

$\therefore \dot{\therefore} \dot{0} 0$

c:

.

:

:

o:

กmn引n

$\operatorname{sm} \frac{0}{0}=0$

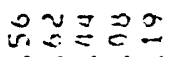

iिं

$1,1,1$

ํำำำ

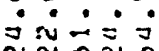

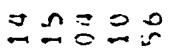

$\because \dot{0} \therefore \dot{0}$

$\div$

승

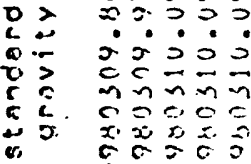

iी

จn $=$ m

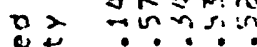

>

ที่ง

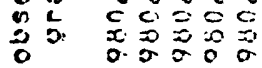

ここちこす

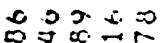

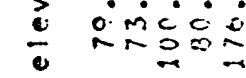

:

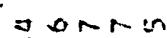

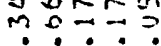
$\dot{2} \dot{2} \dot{0}$

กำำ

$\infty n$ inis. ำ

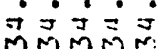
すミすすす

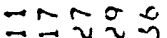
$\dot{0} \dot{0} \dot{0}=$

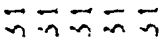

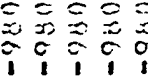
$\ln ^{2} \div \div=0$ $=2-i v$ 行些西

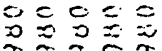

こேこここ कूल $n=3$ $\therefore \dot{0} \dot{0} \dot{0}$

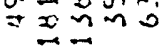

의 यें山ें

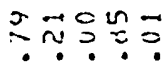
i $\dot{1} \dot{\mathrm{n}}=0$

aथित

$\approx N-m N$

$2 \approx 2 \geq 2$

00 똥 $\dot{0} \dot{0}$

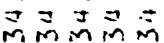

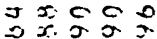
$\dot{i} \dot{j} \dot{=}$ ज्ञज्ञ

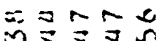
西向的向

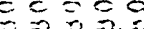
i

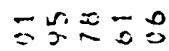

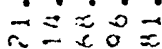
vis

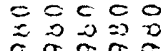

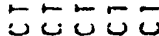
쿵요 语元安远

$\ddot{\Xi} \Xi \Xi す$ 的们的

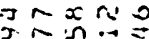
$\dot{\ddot{1}} \dot{0} \dot{0}$ $\approx \sim 1$ ।

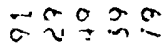

순

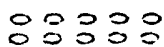

¿ंषं:

푸오을 00000

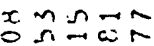

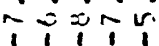

品䟲 $\dot{\therefore} \dot{\sim} \dot{\sim} \dot{s}$ jं0j

只文仝 mine-

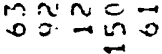

rin $x=m$ iojo

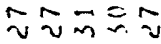
$c<c 0$.

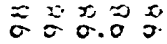

๖ヒここす

ラこேこั

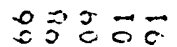
$m=\sin 8$ $\ddot{n}=0=$

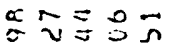
$\therefore \therefore \dot{0}$ 然证 $\sigma=c c=$

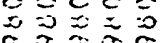

고ㄴㅛㅛ 我衣 ษ!

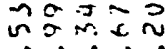
iacis

$\rightarrow 17$

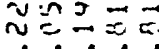
x。․ $1,1 \div$

$\stackrel{2}{20}=$ $n-\dot{s} \therefore$

$x=\cap J \approx$

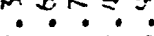
$\ddot{\sim} \dot{\sim} \dot{v}$ viviv

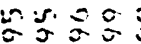
ijoj テニンテ

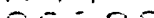
0500

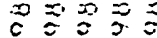
$1,1 i$

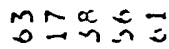
$\dot{n} \dot{=} \dot{0}$ $5 \approx-50$

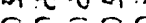

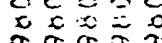

$\xi=2 \tilde{v} v$

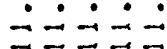
ジンジシ $c 0=c 0$

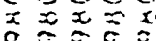
i

$\alpha r m \sigma \alpha$ $=2 m \Omega$ $\dot{B} \dot{0} \dot{0}$ 议证 =0

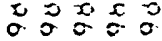

こここここ

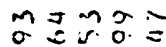
$\therefore \therefore \circ \therefore=$ ำ원

こここここ

뭄움ำ

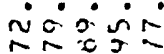
Na西

ㄷำ ㄱํำ $\bar{x} \stackrel{m}{=}=$

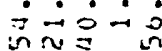

*nNm= 过全总 $m \sim-n \sim$

$\stackrel{\sim}{\sim} \Xi \vec{v}$ $\dot{i} \dot{0} \dot{0}$ $\therefore \dot{0} \dot{0}$ in $\vec{n} 0$

Um ñ

$\sim \vec{\sim} \sim \sim \sim$ ก.T.

웡영

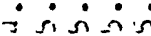

은도 $\dot{n} \dot{n} \dot{0}$ Эミす寻可 ninimin $\because \because \frac{0}{0}$ लिखिंजि অ $\vec{\Xi} \vec{J}$ $\alpha m a=c$

$\sim n m \equiv=$ $\dot{n} \dot{n} \dot{n}$

可可妾
ต $\dot{\sim} \dot{\sim} \dot{0} \dot{i}$

$\sigma] \alpha=4$ $\because \div ?$ 的只出

3033 00000

은 $\therefore \dot{0} \therefore \dot{0}$

$0 \pi v 2 n$ $\because r \Omega a=$

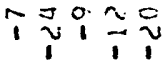
ทีコミ $\therefore \therefore \dot{0}$

ที丶万勺๊ $\therefore \dot{1}=\dot{1}$ $\bar{n} \bar{n} \bar{n} \bar{n} \bar{n}$ $000 \div$ 1 $10 \%$ i

$\Rightarrow m=\equiv m$ บ. กิ: v $v-\approx v$ ग0

บㄴㄷㄴ

I 200 \%

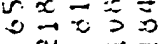
iv $2=$

$\ddot{\sim} \approx \ddot{\sim}$ $\therefore \dot{0} \div$ $\therefore$ एง 72 $m=m$ 11111

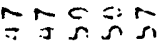
的的闭 उ可可 №m

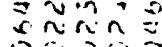
$\vec{n} \dot{n} \vec{n}$ $\because \because \approx=$

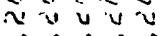
$\dot{\theta} \dot{0} \therefore$

is son is n!

c $n=0 N$

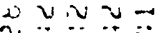
放 20 is it is 4 is nan $=0$. $\vec{n} \vec{n}$ $\because \div \because \because$ ง. v $v$

os inor

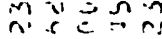

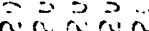
$\exists=$ v v v v $\therefore ? \div \div ?$

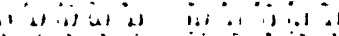

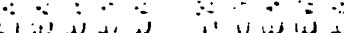

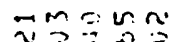
varin $\begin{array}{lll}1 & 0 & 0\end{array}$

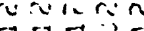
$=\because \ddot{\theta}=$ $-v-u \rightarrow v$ 


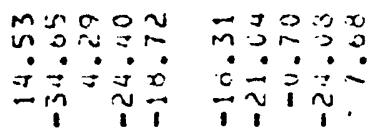

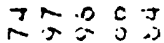

$\therefore \therefore$

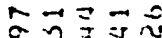

$\ddot{0} \dot{0} \dot{0}$

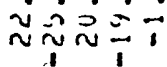

$m \sim \underset{v}{*} \approx$

$0 x x=5$

$\dot{0} \dot{0}$

mini

3영응

33033

○ं0:0

$\therefore \dot{0} \therefore \dot{0}$

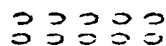

$\dot{0} \dot{0} \dot{0}$

$\simeq \because 0 v v$

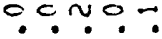

ขึกีำ

$\approx 0 ท 0$ in

$\dot{0} \dot{0} \dot{0} \dot{0}$

0ं0்0

c.

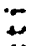

u

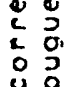

올

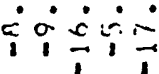

$\vec{a} \approx \vec{x} \overrightarrow{0}$

$B 0$ in

$\therefore \therefore \therefore$ nंग्रं

111

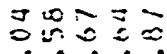

ㅇำ

$\because \approx \exists=5$

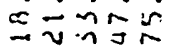

o $v \sim \operatorname{mon}$

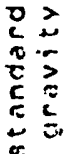

จกกา?

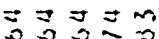

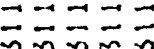

cor

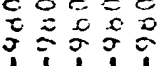

シேシேேே

$=0=0$

可言釷

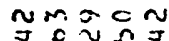

$\begin{array}{ll}3 & 2 \\ 0 & 2 \\ 2 & 2 \\ 0 & 2 \\ 0 & 2 \\ 2 & 0 \\ 0 & 0\end{array}$

$\vec{n} \approx \bar{v}$

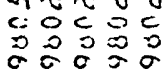

s๐

ここしちこ

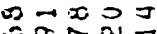

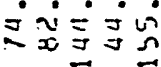

$n 0=07$

$2535=$

$\bar{x}=2-\overline{1}$

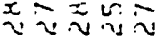

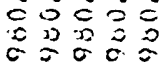

$11 \div$

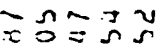

$\dot{0} \dot{0} \dot{0}$

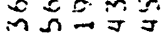

$\Rightarrow \rightarrow r u m m$

$\approx \approx \approx ?$

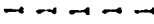

ทัทัทัท

$c=c=0$

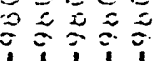

งกัต

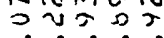

$\rightarrow$ 셍

实的

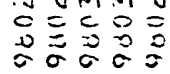

こここすこ

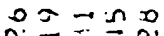

こேここั

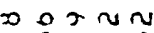

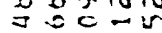

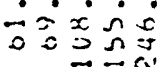

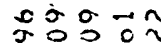

$\sin \sec$

1
0
0
0
0
0
0
0
0

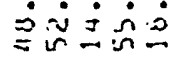

$N=\cong=N$

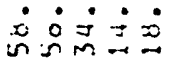

폻

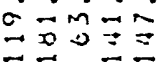

นก

ำำำ

$\therefore \dot{\sim} \dot{\sim} \dot{0}$

MMNMN

$\div \% \frac{1}{1}$

둥요

$x \approx x$ in

$\dot{0} \dot{0} \dot{0}$

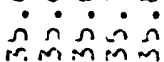

$\sim 0 \pi x$

$x \rightarrow=0$

in $n$ in

可学宁

$\vec{\Xi} \vec{\exists}$

$x-$

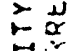

$\geq 0^{2}$

方

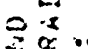

$\underbrace{\infty}_{i}$

는

i)

ث

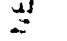

or $x \propto 0$

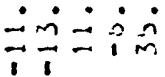

$\therefore \dot{0} \dot{0}$

$\therefore \dot{0} \div-j$

ज可学 $\vec{j}$

紋然品

ỉñ

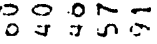

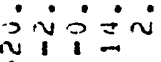

- $\sigma 0 \approx 0$

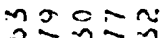

过完

语家

극공영응

30000

$\therefore \dot{0} \dot{0} \dot{0}$

¿̊: $\dot{0} 0 \dot{0}$

จ $\rightarrow v 0$

$\because \because \because \because$

ำ $\sim \vec{c}$

¿ $0 \dot{0}$

$x=030$

00000

$\ddot{x} \ddot{=} \vec{\sigma}$

고요

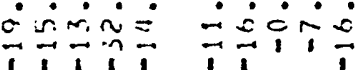

वे ती

$\because \therefore c=$

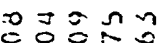

ninoin

mãg

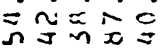

$\dot{1} \dot{0} \dot{0}$

$\overrightarrow{0} \dddot{x}=$

$\because \geq \div \overrightarrow{0}$

$v v v v v$

$\sim \bar{n} \bar{n} \bar{n}$

ooce.

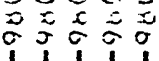

구요

$\dot{0} \dot{2} \dot{\sim} \dot{\sim}$

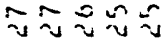

00000

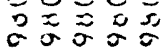

$\approx \tilde{n} \approx \tilde{n}$

$\dot{v} \dot{v} \dot{v}$

$\bar{n} \bar{m} \bar{n} \cdot \vec{n}$

cc: $=0$

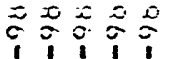

$m \sim N=0$

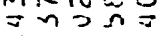

$\because-\div \dot{0}$

vivin in

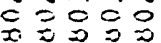

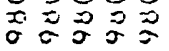

눈

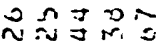

눈효

こニ包包

요음

$\therefore \therefore \dot{0}$

$2 \pi m m=$

กูก

N $\dot{m} \dot{\sim} \ddot{v} \dot{v}$

थnNma

政

inmis

$\because N \cup N m$

$1 \% \%$

m종ำ

$\dot{i} \dot{0} \dot{j}$

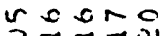

$\hat{N_{1}} \tilde{M} \stackrel{2}{2}$

$\vec{\jmath} \vec{\Xi} \vec{\jmath}$

$\dot{0} \dot{0} \dot{0}$

M $M$ M

可宁荷

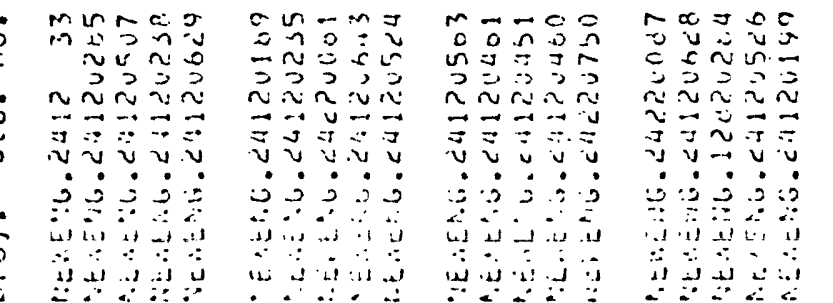

$=0=2 x$

$v \dot{v}=\dot{2}=$

$=30$ 开

$\ddot{\sim} \dot{\vec{i}} \vec{i}$

33230

$\dot{0}: 00$

穴吉向家

$\cong 00 \sim n$

ن

$v v v \sim v$

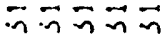

$\equiv=00$

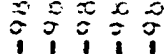

ำรัง

$\dot{0} \dot{0} \dot{0}$

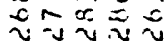

$\begin{array}{llll}2 & 1 & v & 0 \\ 0 & 0 & 0 & 0 \\ 0 & 0 & 0 & 0\end{array}$

宁。合

눈ㄷㄴ

$=\tilde{F}=0 \dot{x}$

$\therefore \cdot \dot{0}$

는

西前 $\dot{\vec{v}} \sim \dot{0}=\dot{0}$

$\bar{N}=\pi N$

$\because \approx 0 \tilde{n}$ v v

$\dot{0}=\dot{0} \dot{0}$

すすす⿹丁口

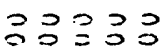

$\therefore \dot{0}=\dot{\circ}$

3323

$\dot{0} \dot{0}=$

ov

$\because \div \div \div$

Nov

نD0்- $\dot{0}=0 \dot{0}$

$\vec{x} \hat{x}=\vec{x}$

$\because \dot{0} \ddot{0}$

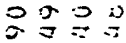

$\beth=m \stackrel{m}{=}$

$\therefore \sim \dot{0}$

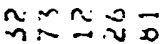

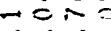

गิ

$\approx 000$

$\dot{v} \dot{v} \dot{v}$

$\dot{v} v \dot{v} v$

$\vec{n} \vec{\sim}=\tilde{n}$

$c=c=c$

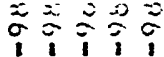

$05 \equiv$

$\dot{v} \dot{\sim} \dot{n}$

$\ddot{n} \bar{n}=$

$=0$.

s.

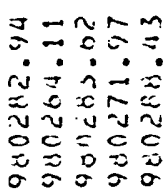

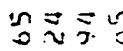

$\therefore \dot{0} \dot{2}$

$\vec{m} \tilde{v}$

$0=\sum=$

둔

叫 $\simeq \Omega$

눈

$\dot{\Sigma} \dot{m} \dot{m}$

$\therefore \Xi \cong \approx$

$m_{i} m x=$

$x=0 x$ in

$\dot{0} \div \dot{\sim} \dot{-}$

可的织

$\cong \simeq \cong \cong m$

$\therefore 05=$

onn

$\because 20=$

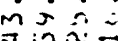

$\div \% \div \%$

$\sim \sim m m$

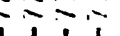

으요 $=0$

ก

cio $\dot{0} \dot{m}$

$\therefore \dot{j} \dot{0}$ 
24

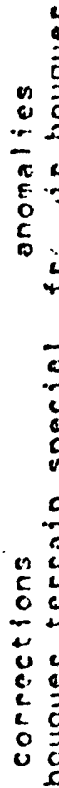

‥ 00030 둥ㅇ

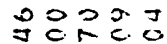
¿ே0்

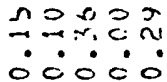

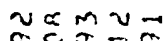

c: $\therefore: \div=$

$2 \pi n=2$ a. $2 \pi, \infty \pi 0$

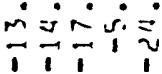

$x$ in $N 00$ corar

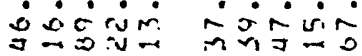

$\checkmark=\vec{m} \geq$ $\because \div \div$

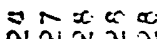

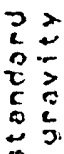

$\sim \sim v \cup$

$\sim m \dot{r i m}$

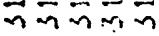

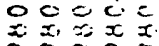

उ.

co요요

$\begin{array}{ll}0 & 2 \\ 2 & 1 \\ 2 & > \\ 0 & 0 \\ 0 & 0\end{array}$

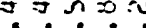

jo $0 \dot{2}$

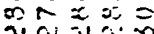

Ũu

$\begin{array}{llll}0 & 0 & 0 & 0 \\ 0 & 0 & 0 & 0\end{array}$

ㄴบㄴㄴำ

$\therefore \Rightarrow x n \approx$

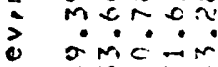

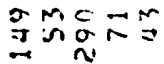

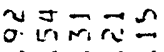

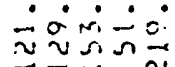

$\sim n \stackrel{m}{=}=$ $\dot{n} \dot{n} \dot{\sim} \dot{\sim}$

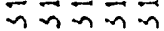

$\because=c=c$

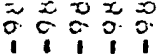

드응 - $\cdot$. $\exists-\tilde{a} \sim$ v v

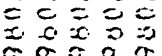

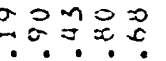

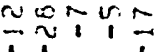

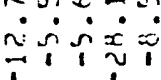

로ำกิก

$\therefore \sim \sim \sim \sim 二$

$\approx=0 \therefore$ $\therefore \dot{0} \dot{0} \dot{0}$

จ N $3: n$

niv $20 \div$

이코 $\therefore x$ in

$\therefore \therefore i n$

$0=0 \simeq 0$

$x=0$ 的

$\dot{x} \dot{\sim} \dot{0}$

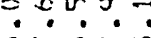

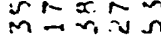

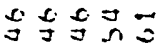

nivin

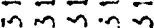

coc: $=0$

वृ 0220

111 !

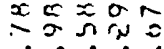

$\ddot{\approx} \dot{0} \dot{v}$

v心u

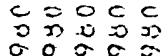

GMNa $\dot{m} \dot{m} \dot{n}$

$\bar{m}=\bar{n} \bar{n}$

$0==0$

x. $\begin{aligned} & 0 \\ & 0\end{aligned}=0$

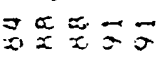

mंn $\dot{\sim}$

minn

c c. $=05$

अ

$0 \sim 000$

$\approx 0 \sim 0$ ᄀ

isi-ja

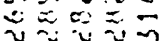

oc c c

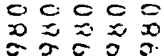

$n=0$

\% ת n

ñn二

$\sim \sim v \sim \sim$

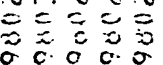

$\approx \sigma \equiv \cong M$

$\dot{n} \dot{\sim} \dot{=}=\dot{\square}$

$m=\bar{m}$

$0=c=$

手示至

रूज

$\sim \vec{\nabla}=0$

$\dot{0} \dot{0}$

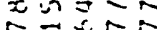

$\sim n v \sim v$

$0=00$

$\begin{array}{lll}0 & 0 & 0 \\ 0 & 0 & 0\end{array}$

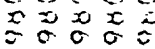

テேேேั

บบธบำ

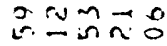

-

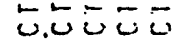

둔두

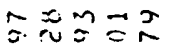

$2=\pi$

ำกㄴ.

- $\dot{0} \dot{0} \div$

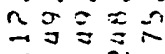

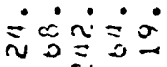

$\dot{n} \cdot \dot{0}$

$m \hat{n}=\tilde{N}$

o돈.

$\therefore \dot{i} \dot{0} \dot{0}$

-

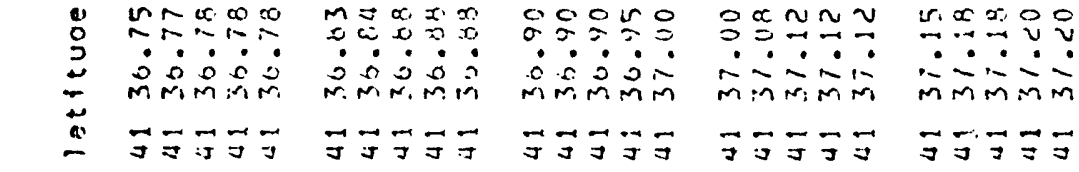

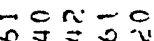

$\therefore \dot{0} \therefore$

Nan

$m m-n r$

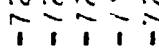

ข้นตำ

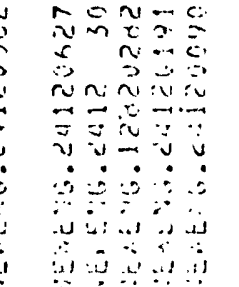

$m=0 \vec{\nabla}$

0.

$\because \frac{11}{11}$

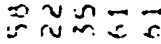

$\therefore \therefore \dot{0}=\dot{=}$

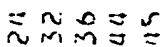

$\dot{\nabla} \dot{\jmath} \dot{=} \dot{j}$

的的的的

$c=20$

111 i

Q

$\dot{\sim} \dot{x} \dot{0}$

$x \cdot x$ x $x$

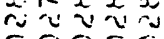

coc $0=$

co 000

5505

-nnn

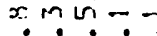

$x \therefore \dot{\sim}$

$=$ in $=$

동 0.

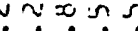

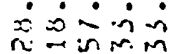

$\because \div \frac{1}{1}$

uncig 完完

mu-n $\Rightarrow 00=0$ $\dot{\sigma} \overrightarrow{0} \dot{m} \dot{m}$

$303 \geq 0$ $\therefore \therefore \therefore 00$

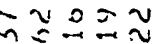
$\dot{0} \dot{0} \dot{0} \dot{0}$

$m=\sigma n n$ o. $00 \%$

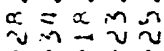
1111

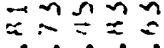
$\therefore \dot{\sim} \div \dot{0} 0$

$\vec{n}=\bar{c}=\tilde{N}$

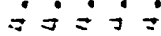
min $\bar{n} \bar{n}$

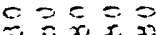

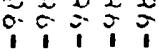

$\equiv m-c 0$ $\dot{i} \dot{0} \dot{0}$

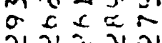
$\tilde{v} \tilde{c} \cong \tilde{v}$

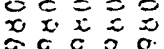

$5 \pm 55$

vnn $=0$ $=\ln \pi \%$ $\sim 300 \%$ nm-n

$m u \sim m$ 0.030
0 $\dot{0} \therefore \dot{0}$

$m \cong M M$ 11\%

J - . . . Minmin mîññm

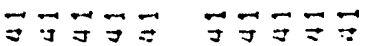

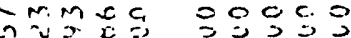

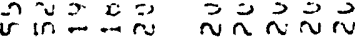

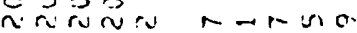

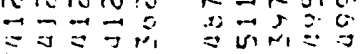

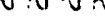

$9 \therefore: 5$ $\dot{9} \dot{2} \dot{0} \div$

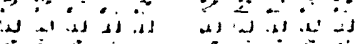

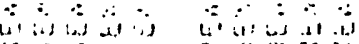


도을

ํํํํำ

$\vec{u}$
$\dot{v}$
$\dot{v}$

을

ن

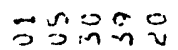

过这

$t^{\prime \prime} 1$

영요응

¿́ं $\dot{0} \dot{0}$

영 330

0.

33393

$\therefore \dot{0} \dot{0}$

กาำ

运金方

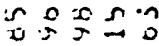

जिं宀:

$=0:$

a

:

กำㅇำ

눈.

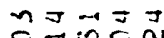

ำ?

$0000 \mathrm{c}$

$x 2000$

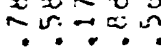

$\approx 2 \pi \cup$

$\div \div \div 1$

设

$\dot{i} \dot{0} \dot{0} \dot{x} \dot{v}$

m $\tilde{n}=$

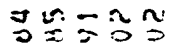

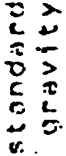

웅

ये

man

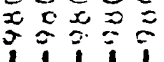

conun

0
0
2
0
0
0
0

$m=\hat{s} 50$

जि:

初的

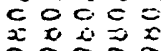

¿0ंí

1

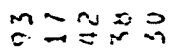

$\dot{n}=\dot{0} \therefore$

ำำำ

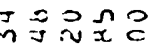

$\therefore \dot{0} \dot{0}$

nos

$\because \approx \sigma \div$

$=\vec{n} 0.2 r$

$\overrightarrow{1} \overrightarrow{1}$

$\approx \dot{\exists} \dot{0} \dot{0} \dot{m}$

$2 \sqrt[0]{2}$

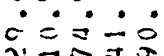

$\vec{a} \approx \simeq \backsim=$

eming

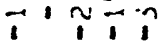

$\approx \dddot{2} \div \div$

$\because \div 9 \div$

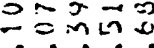

$\approx n=0$

$\because \approx \div \div$

ㅇำ 00

$\dot{\sim} \dot{\sim} \dot{=}=$

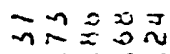

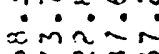

oกำ

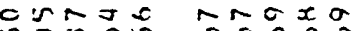

$\rightarrow \exists \exists \Re n$

$\dot{n} \dot{i n} \dot{n}$

$\vec{m} \bar{n} \vec{n} \ddot{n}$

$0=3 c^{\circ}$

$\approx 5 \equiv$

고요

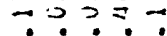

$\dot{\therefore} \dot{0} \dot{0} 0$

กิ๊i

a

\& ธேษ์

ㄸํㄴㄷㄴ

E $\sim \widetilde{q} \approx$

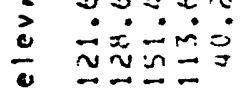

miñ

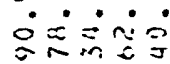

in

necac

$c$
$c$
$c$
5
5
0
0
0
0

$\because \dot{0} \dot{0} \dot{0}$

$m \operatorname{mn} m \infty$

$\because \overrightarrow{0}=\dot{0}$

政-

Nㅠำ

$\sim n m \sim m$

$\underset{1}{n} \cong \frac{n}{1}$

$\dot{i} \dot{i} \dot{\Omega}$

$\vec{\sim} \ddot{\sim} \ddot{n} \vec{n}$

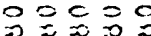

:

c. 3 约 0

$\because ? \div=\because$

$\bar{z}=\dot{0} \bar{i}$

$\bar{v} v \tilde{v} v$

c00 000

$\sigma \approx \sigma a$

こபபㄴ

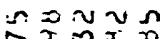

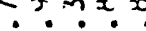

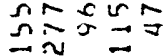

in $\sigma \infty \alpha 0$

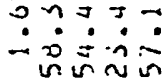

murima

$\dddot{1} \div 1 \%$

ขกง

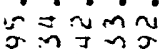

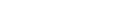

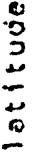

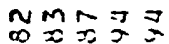

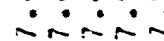

ㅇำ $\simeq$

ำำ $\dot{0} \dot{0} \dot{0} \dot{0}$ mminm

$\vec{\nabla} \vec{\nabla}=$

ज宁可 $\vec{j}$

cocme

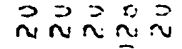

$0=000$

$\vec{i}=\vec{n} \Omega$

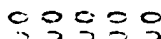

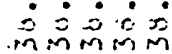

ज可

n证oc

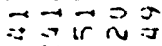

iั $\sim$

$\therefore: 3: 2$

11

is
กั่ง

sonc:

$\because$ MnN

$\dot{3}: \dot{0}$

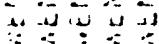

प $m=x \circ \infty$

무루

$\simeq \simeq m m$

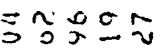

$\dot{0} \dot{\sim} \dot{\sim} \dot{0}$

$\begin{array}{lllll}1 & 0 & 0 & 0 & 0 \\ 0 & 0 & 0 & 0\end{array}$

テテัப்

$? \equiv$ in 0

正出

$\pi \approx \cdots x \geq$

in $\dot{0} \dot{0}$

$\bar{n} \bar{m} \bar{n} \cdot \vec{n}$

co $=0$

$000 x$

ज行

누유요

نं $\dot{0} \dot{0}$

inv v v

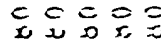

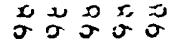

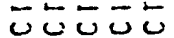

กำก

.

$\stackrel{i}{\because}=\frac{\pi}{N}$

$\approx x=\approx m$ $\dot{i n} \dot{0} \dot{0}$

$\vec{n} \bar{n} \bar{n} \bar{n}$

$0 c=0$

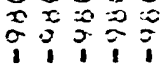

$\therefore \geq m 00$

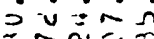

$\tilde{v} \sim n \sim$

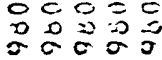

ら兄可

$\sigma: 0 \mathrm{NO}$

in $=0$ sin

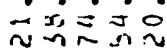

กิกฐ

$\Rightarrow \overrightarrow{7}=0$

? ? ? ?

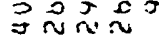

$x \sigma \sin v$.

กวก๊

$\therefore \dot{0} \dot{0}$

NNmRM

munm

$1 \% 1 \%$

$\vec{\sim} \approx \sim \sim ⿻ 上 丨$

$\dot{x} \dot{0} \dot{0} \dot{0} \dot{0}$

$\because=50$

Mिi

$\ddot{\nabla} \vec{\square}=\vec{ज}$

$\ddot{m} \dot{m} \dot{m}=\dot{m}$

ज可吉 $\vec{J}$

EOC00 iñ $2 \vec{n}$

$m=-\pi 0$

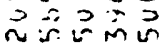

$\therefore \dot{0} \dot{0}$

$\therefore$

S
090
303

$x-x=\infty$

in

$\dot{0}: 3: 3$

دا

is
1. $1 \%$ $\because \approx \sigma \approx$

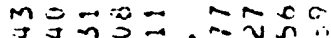
ijij $\dot{0} \dot{0} \dot{0}$

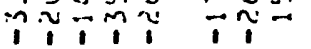

$\Xi=x a \sigma$

$\Omega m=$ $\dot{1} \dot{0} \dot{0}=$ $\because 1 \div \frac{1}{1} \div$

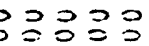

335

$\dot{0} \dot{0} \dot{0} \dot{0}$

0ல்

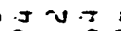

$\because \div \div: \div$

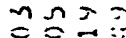

$\therefore \dot{0} \dot{0}$

$\exists \tilde{n} \cdot \tilde{n}=\underline{?}$

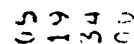

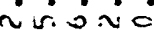

$\div \div \div \div 1$

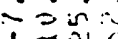

กำ

군?

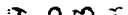

लि马워

$\because \because 0 \div$

$\approx n \leq 0$

$=\nabla \approx n n$ - $-\boldsymbol{u}$. v $\dot{0} \dot{0} \dot{0} \dot{0}$

$\bar{n} \bar{n}=\bar{n}$

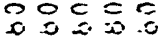

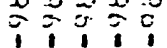

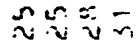

$\dot{0} \dot{0} \dot{0}$

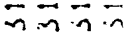

$\therefore 0=0$

i

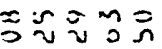

En in $=0$

$\ddot{2}-20$

$v v v v$

c

- 0

$\approx v-$

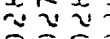

$0=0=$

$55 \div 55$

던

$m v \equiv \equiv$

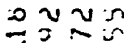

$c=\dot{0}=$

$\Rightarrow$ 되의

ขั0

$I N=r m$

正号

$\dot{\nabla} \dot{0} \dot{v}$

$\dot{i} \dot{\sim} \dot{\sim}$

N $\sim m m$ 


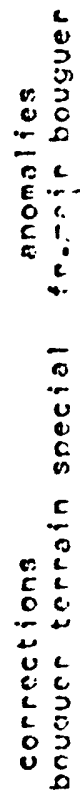

$\frac{2}{8}$

$i$

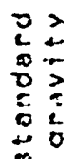

nusino $\because \because \div \div$ co000

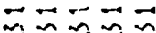
$00 ?$ c. 1 i 1 i

떠요

$\begin{array}{ll}0 & 2 \\ 8 & 5 \\ 2 & 5 \\ 0 & 0 \\ 0 & 0 \\ 0 & 0\end{array}$

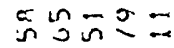
$0=j$ iv

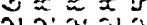
e c c 웡ㅎㅇ

\& ๖こここす

E $\wedge \circ \div=0$

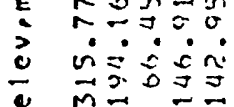

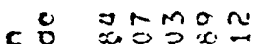
$\therefore \dot{x} \dot{0} \dot{0}$

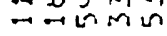

M出只

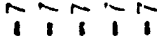

$\frac{8}{3}$

J $35=n$ $\sigma 2007$ $0 x \dot{0} 0$

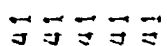

mmis-co $\because \because \because \because \div$ करण丁人 可す寻

$\therefore \dot{0} \dot{0}:$

ก๊ํํำ

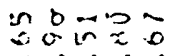

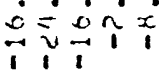

$m \supseteq \approx n \vec{s}$ $\dot{\vec{x}} \dot{\overrightarrow{0}} \dot{\mathrm{i}} \dot{\mathrm{v}}$ $\dot{0} \dot{0}:$ $\therefore=20$ $00=0$ 0
1 过运自

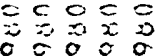
๒ேこ艺 ansa cis Nำ

$x_{x}=0 \tilde{x}$ m $\sim m n \sim n$ 1ิง

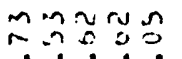

$\stackrel{0}{\sim} \dot{i} \dot{0}=$

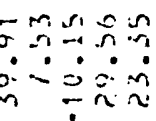

3923

$\dot{0} \dot{0} \dot{0}$

ó $=\dot{0}$

$\Rightarrow x^{\circ}$ $\approx \sim \bar{n} \bar{n}$

EN⿳上m $\because 5=y$. co 0 於的二 c0000

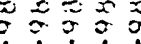

ㄴ. $0 \therefore \approx 5$ ¿ $\dot{i} \dot{j} \dot{n}$ $\bar{x}=-n$ c o $=0$ 末人。

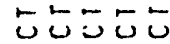
$\operatorname{arn} x$

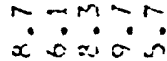
$\stackrel{2}{2} \approx 5$

(1) $\because \therefore ? \div 4$ $\stackrel{\infty}{n} \stackrel{\infty}{\sim}$

N. $=N$ $\therefore \dot{\mathrm{j}} \dot{\mathrm{j}}$

드요 $\dot{0} \dot{\sim} \dot{0} \dot{0}$

ำำ? mะn $11 \div$

m. ก ก ก 111

$\cong \overrightarrow{0}$ $\because v \because=$

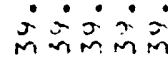

a ก $5: 5=$ minis

ㅇำ $\ddot{\sim} \therefore \dot{\sim} \dot{0}$

शmc日n Sidin

20330 ó0.0

ข己航出 $\therefore \dot{0} \dot{0} 0$ 0 $\infty 0 x-5$ - $\dot{1} \dot{m}$

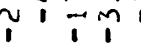
xंن்
N $300 \approx$ $\div \because \because 5 \approx$ $\therefore \dot{0} 0$

mอת N $m=5 \div$ $\therefore \therefore 000$

$n x n=n$

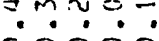

$\therefore 3 \overrightarrow{0}$ r. iviñ

구워 路自

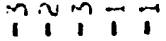

ถูปที่ำ $\therefore \dot{0}$ 프는 $\hat{m}=\hat{x} \tilde{n}=$ $\dot{\Delta} \dot{\dot{0}} \dot{0}$ $\approx \approx N \approx$

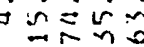

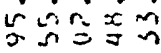

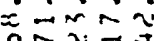
きコすコす
ตก กาก $\therefore-1$.

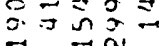

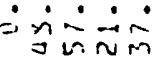
mñm $\div \div \div \div$

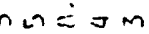
$\because \div \div$

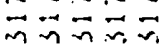
$\Rightarrow 0000$

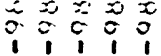

os $\approx$ m $\dot{n} \dot{0}=\dot{0}$ 动此方

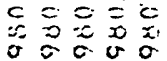

$\approx \equiv x 85$ $\therefore \therefore \dot{0} \dot{0}$ $\bar{n} \bar{n} \bar{n} \bar{n}$ oceco

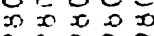
100

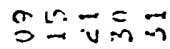
$D \cdot \dot{0} x: 0$ $\bar{m}=\bar{n}=$ $0=0 c 0$

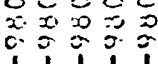

c. sis $0 x$ $x \operatorname{lin}_{\infty}=$ ¿iñ 돈 C 5000

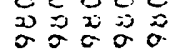

กํㄱ웡응 $\therefore \dot{0}=\dot{0}$

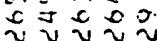
Cec

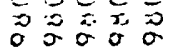

こேちこテ

는

$\sim \sim 2 \sim \sim$

cronis

$\exists \exists=\sin$

品

다중

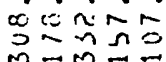

马mgn

$\therefore \dot{\vec{n}} \dot{\mathrm{v}} \dot{\mathrm{v}}$

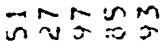

$\therefore \partial \therefore-\dot{0}$

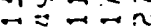

n-mrem m-men

$\stackrel{9}{1}: \frac{N}{1} \div$

corson $\therefore \therefore$ minim

可可可可

$m 0 n m m$ 0.0000

c. MnM $=$

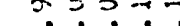

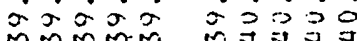

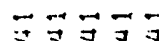

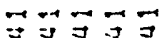

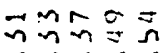
$x x=20$ $\bar{n}=\bar{m} \bar{n}$ coㄷㅇㅇㅛ

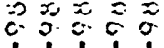
จำจ $\dot{0} \dot{0} \dot{\sim} \dot{0}$

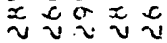
$=0=0 c$

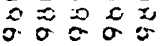

ここちここ

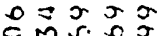
$\dot{a} \dot{0} \dot{0}$

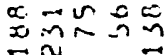

$\stackrel{2}{*} 90 \mathrm{~m}$ $\dot{j} \dot{i} \dot{j} \dot{v}$ $m \sim N$ $M \cap N=\cong$ I $\ln \infty \approx 0$ $\because \because \because 2$ 웡용

$\sin _{2} \sin 0$

$8: 308$

$00=00$ 司公芯

$=c m 00$ n出仙 - mmo...

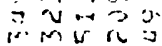
minea os +4 n

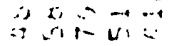

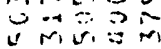
$\therefore \therefore \therefore$ $\therefore \therefore \therefore ;$ $\therefore ; j \dot{j}$ is is is ${ }^{2}$ il $2=\vec{\sim} \vec{\sim} \vec{\sim}$

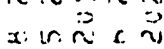
$\rightarrow \Rightarrow-5$ v $\therefore \therefore: 3$ ! 


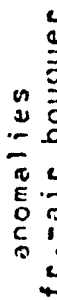

u

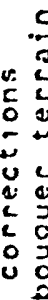

开5

சீं்

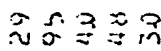

$0 \sin \approx 0$

$D=0$ in

- Moircio

$\therefore x i m a$

i

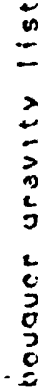

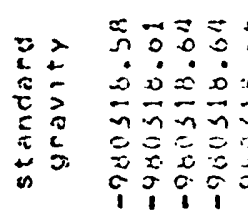

$\begin{array}{ll}0 & 2 \\ 0 & 2 \\ 2 & 2 \\ 2 & 2 \\ 0 & 2 \\ 0 & 1\end{array}$

as

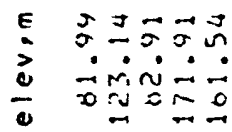

$\exists \approx 0 \leqslant$ in

$\therefore \dot{0} \dot{0}$

$=20=\pi$

ㄷํ워

$\begin{array}{llll}0 & 0 \\ 0 & 0 & 0 & 0 \\ 0 & 0 & 0\end{array}$

ஸேேே

c

ำm녹 $\dot{0} \dot{a} \dot{\sim} \dot{0}$ amño

․․․

U c

วั

ㄴ.

-

$\Rightarrow$ 点

《

$x$.

ב

$2=$

$x \div 2$

A

:

iñ $\simeq$

N츨

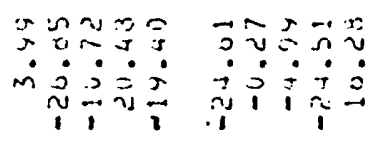

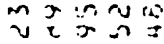

这会它

$1 \%$

$\because \ln =x$

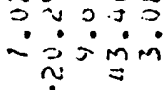

$0 x-7 n$

n $\because \sim$

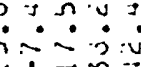

$\dot{i} \therefore \div \dot{i}$

1

00330

$\dot{0} \dot{0} \dot{0}$

ค บ

두웅

vôn

$320=3$

33303

¿वं்:

30303

$\therefore \therefore \dot{0} \dot{0}$

-0்0ல

คำํำ

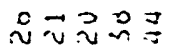

¿́0்

Oंड:0

की

$\because \dot{i v i v}$

$\because \approx 200$

im

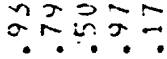

요요

ज 1 M

จ유

$x \div i n$.

$\because \approx m 0 m$

ำ

$\sim \dot{\sigma} \dot{\sim} \dot{\sim}$

isin:

$\vec{\exists}=\tilde{n}$

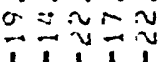

in $N \cong \approx$

$\therefore m \in \&$

$\because ? \overrightarrow{1}$

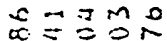

$\therefore \dot{0} \dot{0}$

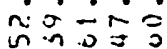

$0 \overrightarrow{2} \approx 0$

$\dot{r} \dot{\sim} \dot{0} \dot{c}$

s.

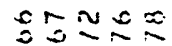

可方尗尗

$m-=0$ in $\dot{0} \dot{0} \dot{0} \dot{0}$

$x=\dot{0} \dot{0}=$

$\bar{n} \bar{n} \bar{n}$

50050

$\bar{n} \bar{n} \bar{n}=$

$5 \equiv=30$

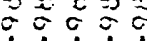

$1,1,1$

ㅇำ经

$\therefore \dot{0}$

两

กv v v

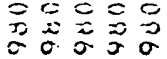

$\sim 7=00$

$-\dot{0} \dot{0}$

ก

COCO?

$\begin{array}{rrr}0 & 0 \\ 0 & 0 & 0\end{array}$

$3=-v$ v

$\dot{\sigma} \dot{0} \dot{0}$

$\bar{m}=\bar{n}=$

$\begin{array}{lll}0 & 0 & 0 \\ 0 & 0 & 0 \\ 0 & 0 & 0\end{array}$

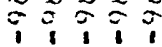

$\approx \approx 0 m=$

$\therefore \dot{0} \dot{0} \dot{0}$

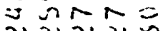

v v v

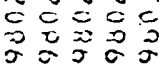

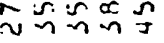

$\dot{2} \dot{2}=\dot{2}$

$\vec{n} \overrightarrow{n_{1}} \vec{n} \overrightarrow{v_{1}} \vec{v}$

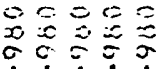

ก气气

$\therefore \dot{0} \div \dot{0}$

$\vec{n} \vec{n} \vec{n} \vec{n}$

ccㄷㅇㅇㅢ

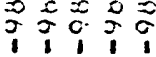

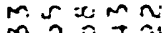

$\therefore \because \div 0$

两: $=0$

$\approx \sim ⿻-v v$

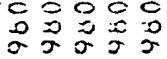

ocnum

$\sigma 000$

$\therefore \dot{x} \dot{0} \dot{0}=$

$n=0$

vข v

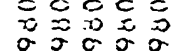

ํํㄴำ

$=\div i \frac{1}{1}$

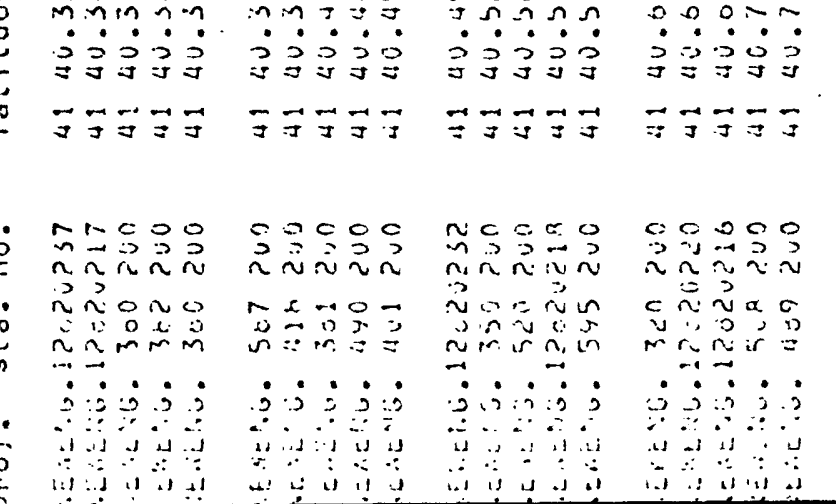

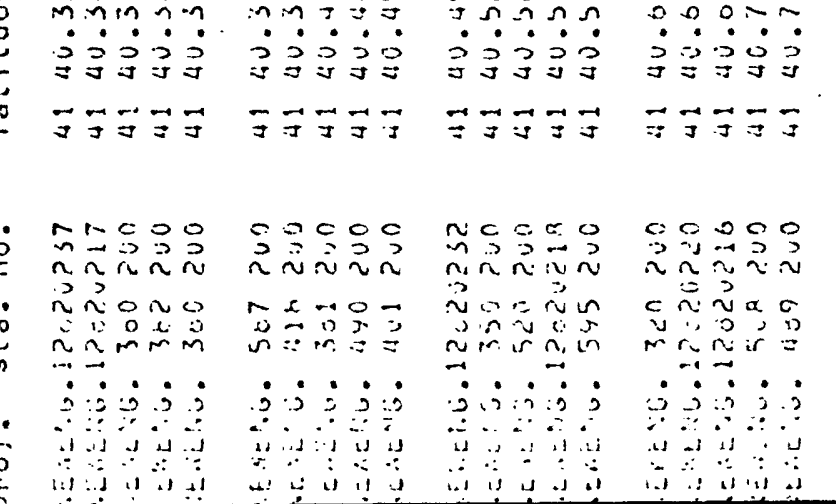

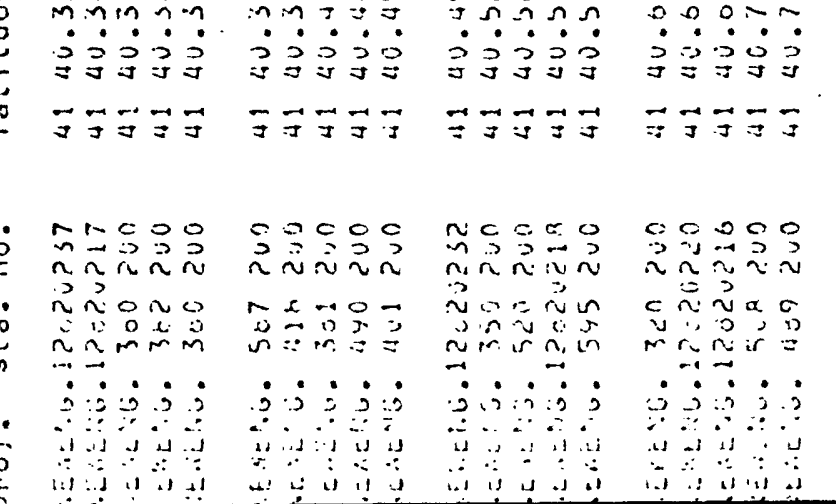

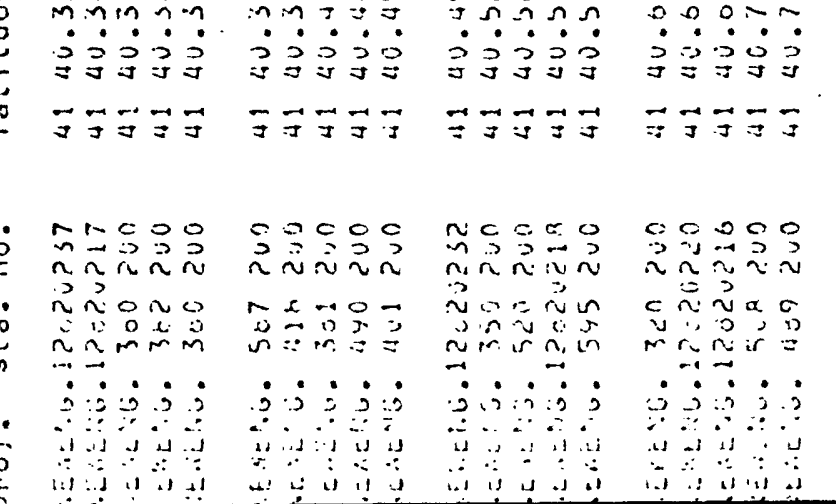

$5-5$

5505

ேேே5

$\rightarrow \sin =0$

rinon

$.020 \pi=$

ニ20可。

ปึ:

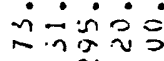

n一⿻

$\therefore \equiv N=J$

$\dot{m} \dot{0}-\dot{j}$

$\approx 0 x$ 눙

$\dot{i} \dot{i} \dot{0}$

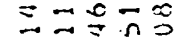

$\dot{i} \dot{0} \dot{0} \dot{0}$

nommo

$\because \simeq \approx m \sim$

$\pi \frac{n}{1} \div \frac{\pi}{1}$

Mथㄹㅇㅠ

$\therefore i \therefore i$

正正正。

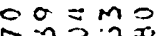

运施充

$m-N m \sim$

$\pi=\frac{\pi}{1} \pi$

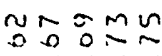

จูลก

ก

.

$\omega m m \approx 0$

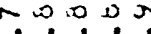

$\begin{array}{lll}0 & 0 \\ 0 & 0 & 0\end{array}$

可焉
テェธ์

$\because \approx \sim m L$

$\therefore \dot{0} \dot{0} \dot{0} \dot{0}$

กับ

$\stackrel{\sim}{\sim} \approx N$

ப்-

MmNNN

$1 \div \div 1$

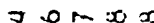

$\dot{3} \dot{3} \dot{3} \dot{3}$

ग $\vec{\jmath} \vec{ज} \vec{~}$

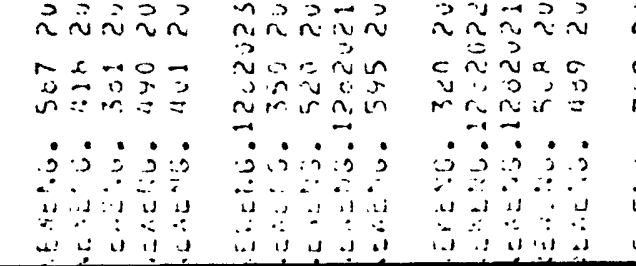

$\overrightarrow{2} \vec{n} \overrightarrow{2} 0$

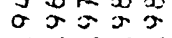

occon o00mL

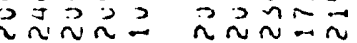

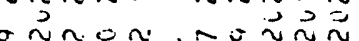

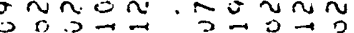

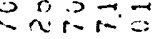

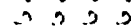
औ $\vec{v} \overrightarrow{0}: \overrightarrow{0} 0$

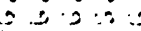

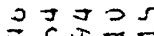
. $\cdot 0$.

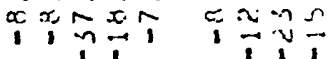

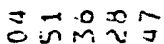
$\dot{m} \dot{1} \dot{0}=$ น

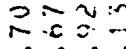
$\hat{\sim} \dot{m i n}_{i}$

$\sim m=\sigma m$ $\Omega 0=0$ $\dot{a} \dot{0} \dot{0}$

$\vec{n}=\bar{n} \bar{n}$

$c=000$

s $\bar{c} \approx \bar{c}=0$

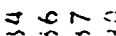

$\approx x \approx 2$

$\sigma \div \sigma=$

$\vec{n} \vec{n} \vec{n}=$

$=c=$

cos

$\approx \approx \curvearrowleft \approx 0$

$\operatorname{ran}$

$\therefore \dot{0} \dot{0}=0$

$n \sim \approx n \pi$

ก v v

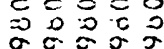

2.

$\hat{n} \dot{\sim} \dot{s}=$

vicive

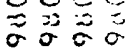

5555

5055

$x>x \sim 2$

$\vec{v} \cdot \overrightarrow{0}$

$c \sim \sigma \pi 0$ mac

됭 $05 m 2$

문

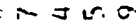

$x_{0}=5$

$\cos 20$

$\dot{\vec{n}} \dot{\vec{n}} \dot{\sim}$

ग. 0 I.

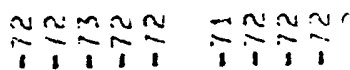

on incen en

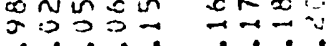

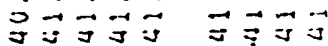

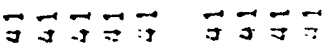

omono

inn $n=n$

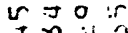

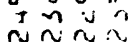

$2=$

$\pi e n$

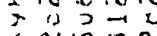

$\sim \underset{c}{n}=$

nin

-

$\dot{0} \dot{0} \dot{0}$

$\therefore-\cdots$ 


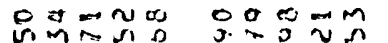

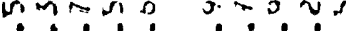

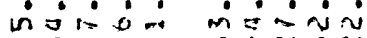

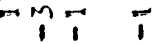

minn

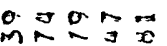

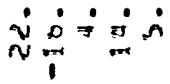

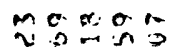

$\therefore \approx \dot{0} \therefore$

$F=\infty \pi C$

$\therefore \therefore \therefore \therefore$

vinirul

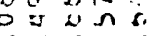

$\therefore \therefore \therefore \therefore$

$\cdots-\because \geqslant i$

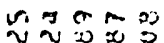

$\dot{a}=\dot{a} \dot{a} \dot{0}$

$\Rightarrow: 0 i \approx$

$\ddot{\because} \therefore \dot{0}=$

11

옹융유융

दंधिं

$20 ? 90$

영요영

¿ெ0்

¿ச்ச்

NoN $B$

$\because \div 0$

正

:

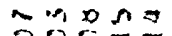

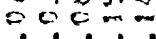

$00=00$

pCo 00

biomentom

$\cos _{0}=\pi$

co000

$\hat{\text { in }} \geq \hat{2}=$ $\therefore \therefore \dot{0}=$

$\hat{c}=j=v$ -ं०ं:

ขึกำ - $\dot{0} \dot{0} \dot{0}$

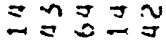
ச்ச0்

Mำ ज्ञ $\therefore \therefore \dot{0} \dot{0}$

ㅇำ

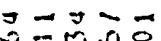

momn ancin

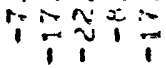

$i \pi i \frac{1}{1}$

- nus

i 111

niviv

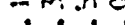

i

mo.m?

os sin

nñ $n$ in ámi $\dot{0}$

11 111

$0 \approx 25 \bar{m}$ $\dot{0} \dot{\dot{0}} \dot{0}$ $\because \div \div \div$

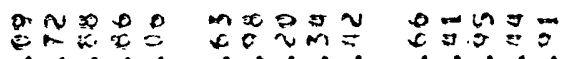

$\dot{0} \dot{0} \therefore$

$\therefore=n \dot{m}$

c.

$\sim \vec{\nabla} \geqslant 0$

$P=\cdots \infty 0$

$v=\sigma r m$

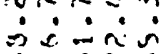

$\because c ? \%$ ?

$\because \div \div$

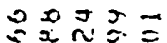

$\because 2 \pi=$

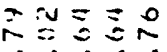

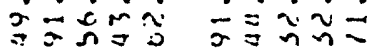

m.

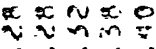

- $\dot{0} \dot{0} \dot{0}$

$\rightarrow \Rightarrow m$ ñ

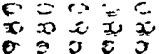

$\dot{=} \dot{2} \dot{2}$

๙ง

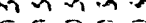

man.

ह 15

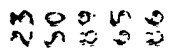

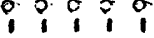

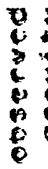

$m \geq v 1=$

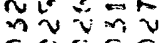

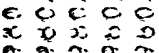

Se $5:=$

$\therefore \therefore \dot{0}$

ข

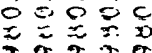

可它芯

$=5=$

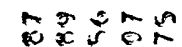

$\exists x^{2} \geq m$

- $\begin{gathered}0 \\ 0\end{gathered}$

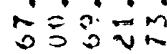

约

0

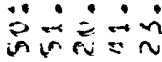

บ $0=\frac{2}{2}$

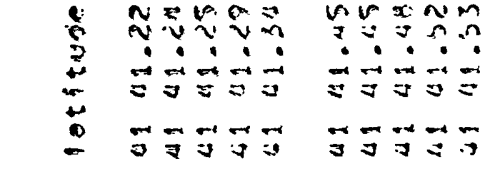

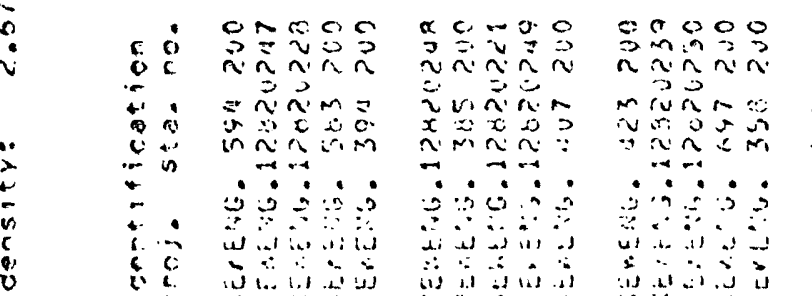

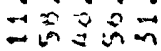

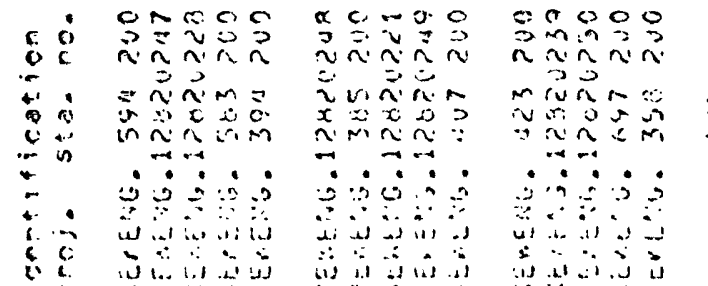

nts

co

के

$\because 2$ a

$10 x=2$

v v v

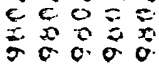

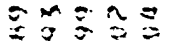

$\dot{2} \dot{2} \dot{a}$

muñ

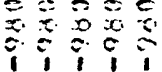

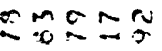

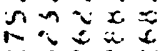

ำขปัป

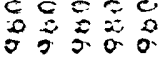

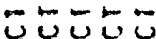

$5-505$

is $=0.0$

onnio

₹०\&in

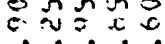

$\therefore=20$

ะับเก็

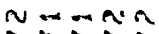

$7 \% 1 \%$

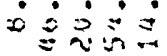

$M_{1}^{N}=\frac{N}{1}$

$\stackrel{c}{c}=2$ in

5ำ

$\dot{0}=\dot{1}$

va

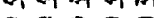

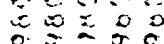

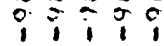

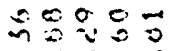

juvar

v.n vũ

$\begin{array}{lll}0 & 0 \\ 0 & 0 & 0 \\ 0 & 0 & 0\end{array}$

의

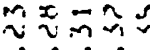

$\vec{n} \ddot{\sim} \vec{\sim} \ddot{n}$

ที่กต

$\approx 00=$

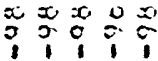

$m=n=m$

$\div ? \div \% ?$

$=-\cdots v$

vñn.

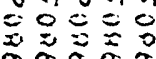

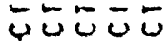

: ヘN心

nom

过它

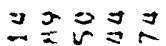

- vins

$\checkmark \lim _{0}$

กคก ก๊

$\vec{a} \vec{\imath} \vec{\sim} \vec{\imath}$

กิกีุ

$\circ 0==$

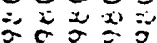

i

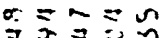

$\therefore m 5=0$

ขิง

이일

들 $=$

$\vec{n} \ddot{n} \vec{a}=\vec{a}$

$\hat{c} \cong \tilde{c}=\tilde{c}$

जेष

$N \dot{n}=\tilde{v}$

$\therefore \dot{0}=0$

$-c=2-$

는

$0=50$

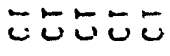

วฐกิ์

$\dot{0} \dot{\sim} \dot{r}$

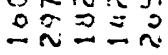

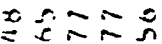

$\therefore \ddot{r} \dot{s} \dot{0}$

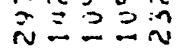

or 0 in 5

$M M N M \cong$

$\because \cdots \div=?$

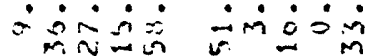

حCN

ร $=\frac{1}{2} \div$

$m \equiv \equiv \equiv$

$\dot{\sim} \dot{n} \dot{n} \dot{v}$

añn

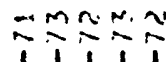

$\sim m \cdots m$

$\dot{v} \dot{i}=\dot{\sim}$

$m \sim-\ldots m$

$1 \% 1$

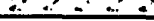

$\because 50 \nabla 0$ जिते

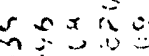

is

$\therefore \therefore ;$

$\therefore$ iा $\therefore$

$\therefore$ is $\cong$ ๖す๊

$\dot{i} \dot{i} \dot{v}=$

$0 N=n$

$\underline{v} \dot{v} \dot{v}$

苛羿宁
ำำ

$\dot{v} \dot{v} \dot{v} \dot{v}$

$\vec{\nabla}=\vec{J} \vec{\nabla}$ $m \equiv n \sim \equiv$

$\therefore \dot{v} \dot{v}$

す岌すす

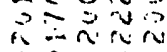

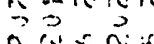

$\therefore \Rightarrow$

政的。

$\because \therefore \therefore ?$ conco

ते

Na $\ddot{i} v \vec{v}$

mis:

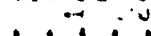

이요

c) $=00 c$

nNei

人)

ñ

$\Rightarrow \vec{n} \vec{\sim} \vec{n}$

- $\because$

. $\overrightarrow{0} \cdot \overrightarrow{0}$

$2 \div: ?$

$? \because 3: \therefore$
ว $2=0 n$

x $\begin{array}{llll}0 & 0 & 0 & 0\end{array}$

(2)

$\overrightarrow{2} 0=0$

जิ กิน 
ñus

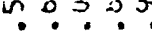

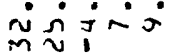

$\cos 20$

ñ⿻

$\dot{v} \sim \dot{0} \dot{0}$

îm.

잉으응 $\therefore: 000$ 00000

NนAD กnn C0000

$\vec{u} 0 \hat{0}=0$
$\dot{0}=\dot{0}=\dot{0}$

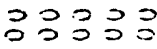
¿०0ं0

공영 $\dot{0} \dot{0} \dot{0}=\dot{0}$

30393 $\dot{0} \dot{0} \dot{0}$

กำ $\Omega \simeq$

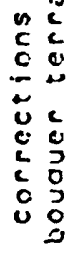

M̃N口

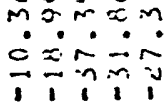
जo - $\dot{1} \dot{0} \dot{0}=$

$2 \rightarrow 2=2$

$? \sim r=?$ 舟可至

จ $5: \div \div$ $\approx \Omega \approx x \sim$ กヘニッ

$\equiv N \approx \frac{\pi}{x}$

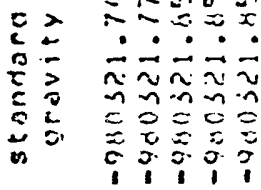

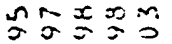
$\therefore \dot{0} \dot{\sim}$ $\approx \sim \sim n j$ c c $=0$

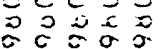
ज行

$=0 x x \sim$

$\begin{array}{ll}2 & 2 \\ 3 & \vdots \\ 2 & 2 \\ 0 & 2 \\ 0 & 2 \\ 0 & 2\end{array}$

$\because \div$

요물

$v N v \tilde{v}$

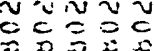

ます心

* 5ேこ 20 $0=2$

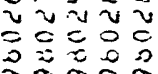

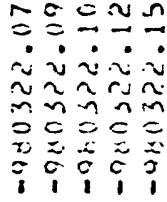

$3 ? 2 \ln$ $\dot{0}=\dot{2} \dot{1}$ तi

ำกำก aćxi

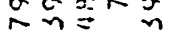

$\because \tilde{n} \approx \tilde{r}$

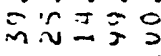

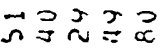
$\dot{0} \dot{0} \dot{0} \dot{c}$ 1. I

$\because n 20=$ $\dot{x} \tilde{i} \dot{0} \dot{0}$ $\overrightarrow{1}, \overrightarrow{1} 1$

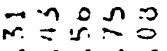
$\ddot{\sim} \dot{0} \dot{0}$ IN $\frac{\sigma}{n}=0$

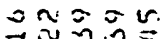
$\dot{j} \dot{i} \dot{\sim} \dot{v}$ $\approx \tilde{n} \tilde{n} \tilde{n}$ วn๊ำ

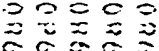

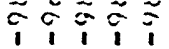

$\exists=0 \approx \Omega$ $\vec{j} \approx=0$

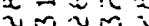

$20=0$

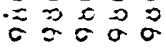

ก? ? ? ? $x x=20$

$v \sim \sim \sim v$

$\begin{array}{ll}0 & 0 \\ 0 & 0\end{array}$

จำ $\dot{v}-\dot{v}-\dot{j} \dot{v}$

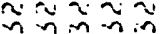

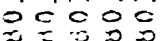
$\approx \equiv \approx 00$ ज 595

$\sigma \tilde{\sim} \sim \sim 0$ ทำ บ $\approx \sim \approx N 0$ งิ่v一

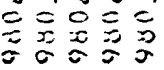

ㄴํㄴㄷㄴ 콘

눈

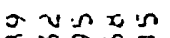

$m \geq 00 n$

$\dot{\sigma} \approx \dot{0}=\dot{0}$

$\therefore \approx \approx \sim ⿻ 上 丨 寸$

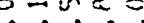

$\therefore \approx \approx \sim ⿻ 上 丨$

ㄷㄴㄴㄷㄴㄴㄷㄴ

van

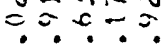

$\therefore \sim \dot{\sim} \sim \dot{\sim}$

U $=N v$

テேே5

$n=\pi \approx n$

$\dot{0} \dot{\vec{j}} \dot{\mathrm{s}}$

กำ

$\sigma \approx \sigma 0 \approx$

a c a r n

$m \approx \sigma=N$

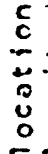

$\ddot{0} \dot{0} \dot{0}$

$\approx \simeq m m n$

$\sim \dot{\sim} \dot{0} \dot{0}$

$\div$ กำ

주의

$\exists \approx \simeq 95$

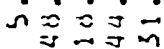

$\sim N M M-$

MNNNN

$\because \div \hat{1}$

$\because N \cdots \cdots=$

$2 \div \frac{2}{1} \pi$

$\therefore \dot{0} \dot{0} \dot{0}$ mun.. n

$\sim \check{N} \cong$

2)

แU

ب̂:

空总

岁:

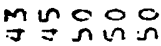

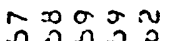

n $x+\infty 0$ $\ddot{v} \dot{\sim} \dot{\sim} \tilde{v}$ iviviv

可岗可

$\vec{\Xi} \vec{ज} \vec{J}$

$\begin{array}{lll}1 & 0 & 0 \\ 5 & 0 & 0\end{array}$

$\dot{v} \dot{v} \dot{v} v$

$=$ in $0 \div 0$

$\dot{0} \dot{0}=\dot{m}$

นกน

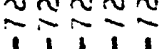

可䒚志

$\dot{v} \dot{v} \dot{v} \dot{v}$

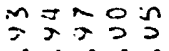

$\stackrel{i}{j} \dot{j} \bar{i}$

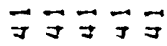

䒚宁可

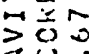

$x=n$

$\Rightarrow \equiv$

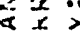

w.

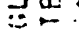

- 0000

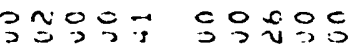
นับณณ in $\vec{\sim}-n \vec{r}$ in 200 $\sim \sim 20 \simeq$

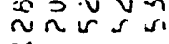
$\because \div 3: \dot{0}$ urnna nกn织 ñ. ñ. ij; $;: 3$ <.

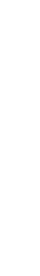

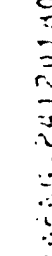

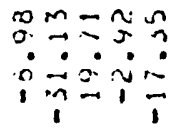

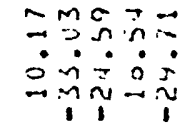

$\bar{n} 2500$

$\therefore \dot{\sim} \dot{\sim} \dot{\vec{v}}$

$\tilde{v} \approx 00 \%$ $\dot{i} \dot{\sim} \dot{\sim} \dot{0}$

$m \sim \frac{\pi}{1}$

至的里:

$\dot{0} \dot{1}$

$\overrightarrow{1}$

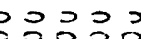

․․․․․

$\because 2303=$

$\because 2 . ? 3$

00000

293

$000=$

ก J气ํำ

$n$

กํํㅁㄴ? -ं0்:

$\cong$ กำ 00000

$\exists \equiv m \cong n$

s.

กำก

$\therefore \dot{1}=\dot{1}$

o $=10$

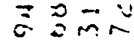

aim

x-

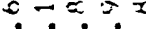

$\dot{m}=\dot{v} \dot{\sim}$

$\cong \geq \hat{n} \overrightarrow{0}$

$\therefore \dot{0} \dot{0}=$

50

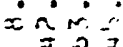

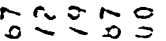

ñ

=0ํำ

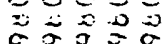

c:

$O E M O$

$\because \div ?$ ?

$\approx \approx v-\infty$

$\therefore v \tilde{m}$

$c \equiv \equiv c c$

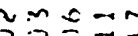
$\dot{\sim} \dot{\sim} \dot{\sim} \dot{\sim}$

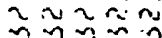
$=c c c=$

$\dot{z} \approx \approx x$

i

$\bar{n}=\mathrm{n}$

$\dot{v} \dot{\sim} \dot{0}$

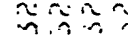

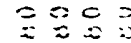

$1: 3$

0 no

․․․

นn mo:

$x \geq x$ 的

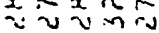

$\sum_{0}^{0}=0$

:

$05=$

$\ddot{v} v=$

$c=0 c$

可可可

525

$\exists m \bar{c}$

$\dot{0} \approx \dot{\sigma} \dot{3}$

$\underline{2} \approx \cong$

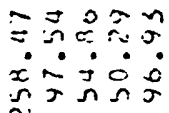

눈

in $50 \approx n$

$\because \cdot \dot{0}$

innmin

$\sim \sim \sim \sim N$

$\simeq \simeq \simeq \because$ 
:

$\bar{\pi}$ us

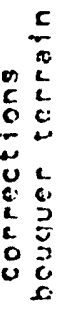

$\therefore \vec{c} \sigma \vec{\sim} \vec{\sim}$

$\because \because \because \div$ $\therefore$

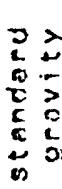

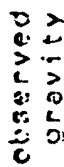

mas

min $\dot{m} \dot{0}$ sneñ cद $=0$

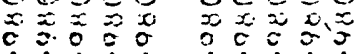

in $m=s$ $\therefore \because \div$ - $5=$

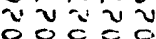

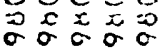

เ ヒேしむら

E $x=2$ in

$\Rightarrow \quad$ inisio

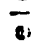

\begin{tabular}{r}
$c$ \\
0 \\
0 \\
0 \\
\hdashline 0 \\
0 \\
0 \\
0 \\
0
\end{tabular}

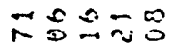
ति $\dot{x}=\dot{m}$ $\therefore \dot{\therefore} \dot{\sim} \dot{0}$ Noma $\because 2 \pi \frac{1}{1}=$ $\because \pi \hat{1}$

نํ.

$n=\pi x$

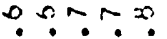
inini

$m \leq \approx n$ $\because x: 2 \div$ $\dot{\sim} \dot{\sim} \dot{\sim} \dot{n}$ $\checkmark$ ป⿻

すすすすコす⿹

$\approx v=00$ $\therefore \div \div \div=$ mn=m xin.on

4. $0.0 M m$

ว.? $v ?$ ? $\Rightarrow \tilde{n}=0$

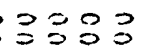
$\dot{C}=\dot{0} \dot{\circ}$

ming $\therefore \dot{0}=\dot{0} 0$

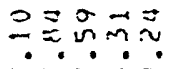

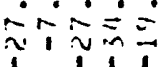

ตำกร - $\dot{0} 0 \dot{0}$

$\overline{=}=020$ Cं்

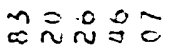
0ं்

चm $=5$ ¿๐் 0 :

兄元品焉 Oं் $\dot{0}=$

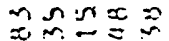
$\ddot{\alpha} \dot{v}=\dot{n}$

$\because N C N$ วนบ? ? 证究 $\because, \cdots n \sim \cdots$ c c c c c c $\approx 0 \approx \approx=0$

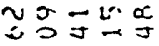
$\therefore \dot{0} \dot{0}$ $\sigma n=n$ ข vขv $0=03=$

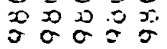

ஸே兀 กับำ

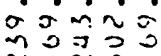
- I n n

$m=a=0$ $\dot{0} \dot{0} \dot{0}$ mamima $1 \div 1 \%$

$n c n=s$ $00 \div-1$

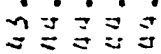

ज可可

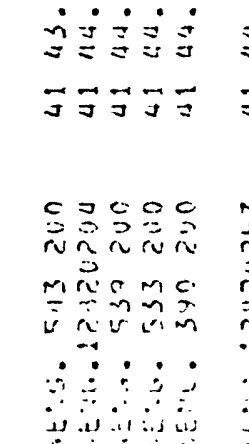

$n=\vec{s} \pi$ $\div \div \div 0$

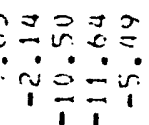

$m \overrightarrow{0}=\vec{z}$ $\dot{v}=\dot{\sim} \sim$

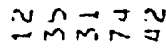
$\therefore \dot{m} \dot{m} \dot{x}$ 11

ำำ

D保年D $\therefore=5 \div 0$ in $\sin 0$

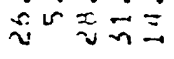
$\exists \check{\sim} \dot{\sim} \dot{0} \dot{0}$

घç冖a 의 $=0$

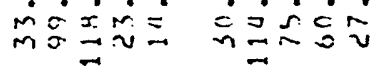

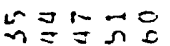
$\dot{\vec{j}} \dot{\vec{n}} \dot{\vec{n}} \dot{\vec{n}}$ $n \Omega \approx n$ occ c c o

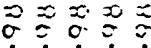

$n \approx 00 n$ $0=0=\pi$

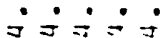
$\vec{\sim} \vec{\sim} \vec{n} \vec{n}$ $\therefore \in 000$

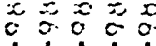

$n=\Sigma=N$ …… $\therefore \dot{a} \dot{3}$ $\because \approx n \approx \approx$ 0 : 00 $x=000$ i i i i

$\pi-\pi v$ $\therefore \dot{\sim} \dot{=} \dot{1}$ nn

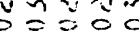
o 0 \%

$\sigma \cong m \vec{J}$ $\dot{0} \dot{i} \dot{0}$ $5=0$

ข $น v ข$ o.

$\approx 二 \nabla=5$ $\therefore \dot{0}$ $\approx \approx=1$ v $v=n$

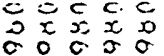
ธேบับับ テับับั 둔 $\approx 0 \div 00$ $\because \because i s i m$ anm?

$\dot{i} \dot{0} \tilde{0} \dot{0}=$

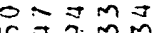
ก. J m mexis

CNL

쿠요 $\dot{0} \ddot{0} \dot{0} \dot{0}$

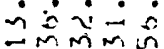

-n min N $\approx \approx \simeq \sim$

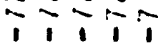

$=\pi \ln$

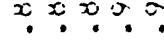
$\vec{\nabla} \vec{u} \vec{n}$ $\rightarrow$. oc 0 वैक 1 i i i

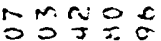
$\dot{\square} \dot{=} \dot{=} \dot{0}$

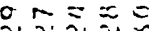
$c=000$

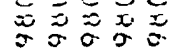

넌

$\Rightarrow n \sim \approx n$

$0.0 .21 \%=$

cinar

añ

공ำ $\therefore \sim i 0$ ज्ञ

$0 n-=$

$\div 520$ กน乞 o 000 1111

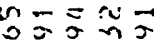
$\therefore \dot{\sim} \dot{0}$ $\sim \approx 25$

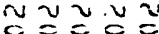

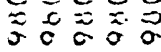

氙河

$\eta \vec{人} \cong \approx$ 0
4 을용

$\Rightarrow \approx \approx 50$

$\infty$ nc $c=$

$\infty \sigma \infty>m$ $\therefore \dot{i} \dot{0}$ $\because \cdots$ $\dot{i} \dot{v} \dot{j} \dot{v}$ mamn. $N M \cdots-N$ NMM N 1111 $\because \% \% \pi$ 11111

$\sim m \ln a$ c) $5=0$ nmmin cnun s. arust:

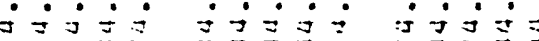
$\vec{\nabla}=\vec{\nabla}$

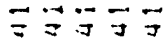

MOCOC $\hat{n}=\ddot{n} \vec{n}$ $\vec{v}=e \cdot r_{1}$ is

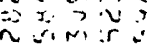
$\because$. $\because \because \frac{0}{0}$ $n \cdots=n$ $\overrightarrow{2} \overrightarrow{2}$ $\because \simeq \approx \approx c$ $u=\vec{r} \cdots$ $v-2$

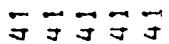

coc.00 $\therefore \vec{r} \ddot{n} \dot{n}$ aris in

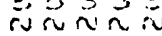
som$\dot{y} \dot{i} \dot{j}$

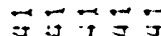

$\therefore \dot{\square} \dot{\square}=$ 可寻寻 $\ddot{\Xi} \ddot{\jmath} \ddot{\Xi}$

OCc c.

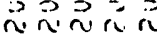
(r) $\sim \sim n=$ uve vin $0=0=0$ $\ddot{i} \sim \ddot{\sim} \dot{\vec{n}}$ marso in: $y=n: m$ 
2
0
0

$m=\sigma 0 \sim$

जेंvن⿺

$\cong \overrightarrow{050}$

in $\dot{\dot{s}} \dot{\dot{1}} \dot{-}$

$1 !$ !

$\because \dddot{1}$

웡ำ

in $\dot{\vec{v}} \dot{\vec{v}} \vec{i}$

v음

$\dot{I} \dot{\sim} \dot{\sim} \dot{\sim}$

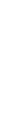

กำํำ

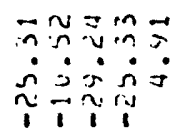

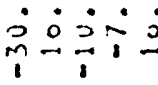

งกา

ก:ว.?

ñein

ข

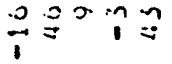

$\vec{i} \dot{0} \dot{i}$

응ㄱㅇㅇㄱ

03230

¿00:0

영

39533

$0323=$

?. ? ?

$0.9=3: 3$

20303

¿0ं0்

30333

¿¿0:

32039

- $0 \dot{0} 0$

응은

$\therefore \therefore 0$

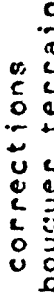

$\sigma a n$ in

둥웅

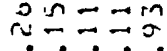

$\therefore \therefore \div 00$

añ

$\underline{*} \div$

- 000

ชที่ก๊

$=0 \therefore \dot{i}$

$\overrightarrow{11} 1 \overrightarrow{1}$

기요

$\sim \approx 0 \mathrm{~m}=$

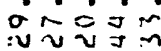

$\approx 000$
$\approx 000$

$\overrightarrow{1} \overrightarrow{1} 1$

$\Omega 0 \approx \sim r$

$\simeq 00 x x$

ง v

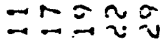

$\begin{array}{ll}3 & 2 \\ 2 & 2 \\ 0 & 3 \\ 5 & 0 \\ 3 & 2 \\ \text { in } & 0\end{array}$

$\dot{\hat{n}} \dot{\hat{n}} \dot{\hat{n}}$

กำn

$0=200$

द श

$n=n 0=$

$\begin{array}{ll}= & 2 \\ 0 & 2 \\ 2 & 5 \\ 4 & 0 \\ 0 & 2 \\ 0 & 5\end{array}$

is

E. ก๊ก०m

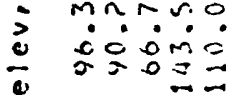

ํㅡㄴ

บับำ?

$\simeq-\sim m s$

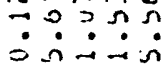

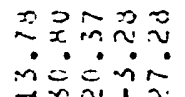

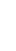

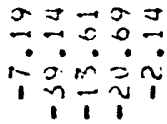

ก๊ำ

จำำ

$\therefore \dot{\sim} \dot{\sim} \dot{0}=$

$\therefore \dot{\Xi} \ddot{0} \dot{\Omega}$

证

$\therefore \dot{i} \dot{\therefore} \dot{0}$

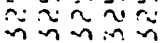

$0=0$
$0=0$
0

\% $5 \div \overline{1}$

$m \cong \vec{n} \vec{D} \cdot \overrightarrow{0}$

$\therefore \therefore \dot{0}$

$-v \tilde{v} v$

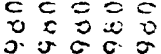

c유 กิน ñ $\tilde{\sim} \tilde{\sim}$ $c=20 c$

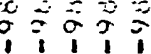

ñc⿻一𠃋十

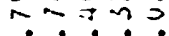

$\therefore=j n$

$\sim \approx v_{11} \tilde{v}_{1} \tilde{v}$

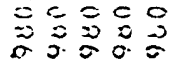

ज5

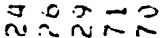

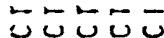

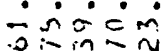

$\widetilde{0}=2$

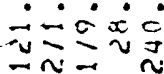

$=0$ in

$\dot{0} \dot{0} \dot{0} \dot{0}$

$n \approx n \sim 0$

DCO $=5$

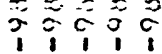

máco

v $n=n$

Din

$\alpha=\tilde{v} \tilde{v}$

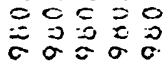

$52=2$

$0=\pi 00$

acoind

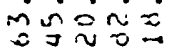

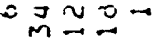

nm nm

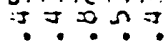

$\dot{v} \dot{j} \dot{\sim} \dot{m}$

ร 0 요

$\rightarrow$ กำ

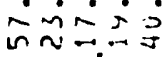

NNNNN

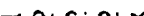

$\sim m \sim \sim m$

$=m \simeq \approx \approx$

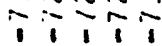

$17 \% 1$

nJm

n $N \cdots$

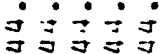

菏学

$\Longrightarrow=0 \simeq$

$\exists=\tilde{m} m$

x in $=5$ in

ㄱํㅇ $0 \hat{0}$

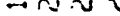

$\ddot{n} \hat{n} \dot{n}=$

กُ

$\vec{\Xi} \vec{J} \vec{\jmath}$

ज可菏

可可

o n

ये̃ñ

$\overrightarrow{3} n=\vec{v}$

an $=20$

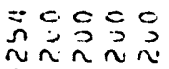

융영응

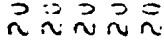

$\vec{r}=\ldots$. n

$\sim: 0=\approx$

กำ $2 \pi$

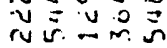

90599

nuñ

$r=0, \tau$

j:

$\because \dot{0} \dot{0} \dot{0}$

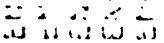

$\therefore \therefore ; \vdots ?$

Mr $\rightarrow$ is

50900

$\vec{\sim} \vec{\sim} \vec{\sim} \vec{n}$

$\sigma x \cdot 0 \sim=T$

Ninmin

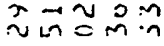

نं००००

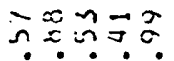

$\because \operatorname{cin}$

11

군은?

穴亦采向

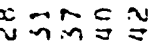

$\dot{0} \dot{0} \dot{0}$

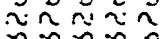

$\cong \cong 00$

i

Э

$\dot{v} \dot{v} \dot{0} \dot{v}$

$v \tilde{v} \bar{v}$

0
0
0

은? 00

कि: :

รูกษ

$\dot{2}=\dot{1} \div \dot{0}$

\%1

$\therefore \approx D \sim$

$\approx-r=0$

$\because \dot{\sim} \dot{\sim} \dot{\sim}$

$\approx \cong \bigcap ? \vec{n}$

Li் $\dot{0} \dot{0}$

กิ

ᄂง

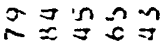

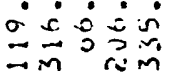

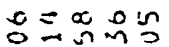

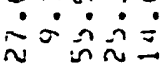
$M M=N M$

0 의 in

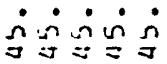

م $\approx=$ $\therefore \dot{0} \dot{0} \dot{0}$ $\sim \sim \sim \sim \sim$ 응응

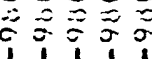

1111

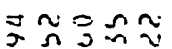
$\dot{2} \dot{0} \dot{0}$

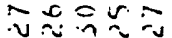

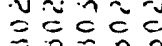
$\approx 0 \approx 0 \%$

5505

กิ $0 \cdots p=$ $\dot{a} \dot{\dot{0}} \dot{0}$

nঙ心

nxmmo $\because \dot{m} \div \dot{0}$ तथ $\vec{m} \overrightarrow{0}$

$\cong m \cong N$

กิำำ

$\dot{0} \dot{0} \dot{0} \dot{0}$ $\sim \sim \sim \sim \sim$ กทนทั $\equiv \equiv \equiv$ र्रा

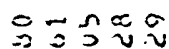
$\dot{\nabla} \dot{i}=$ c $\vec{z} \approx n$ กัป a d

$-2-2=$

글

$\dot{0} \ddot{0} \dot{0} \dot{0}$ $=n$

กิ궁 กลว $\therefore \dot{0} \div$ $\sim \pi \sim n$

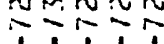

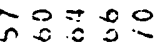
$\dot{0} \dot{0} \dot{0}$

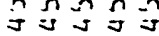

Nบำ $\dot{0} \dot{0} \dot{0} \dot{0}$ 可可寻

coou a ñ山ñ $=0 \operatorname{con}$

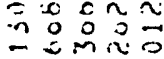

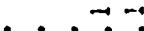
:3:3:

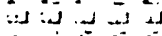
ثـ

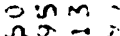
$\therefore \dot{v}$ N

च告

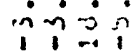

noin? $\because \because \div=$

눈= $\therefore \dot{0} \dot{\dot{C}}$ 


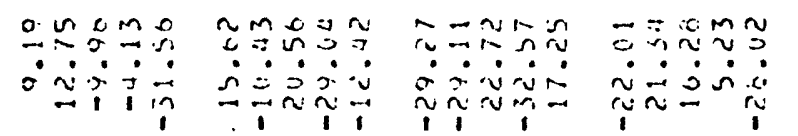

Nas

$\dot{0} \dot{0} \dot{0}$

mคि

궁영요응

$\therefore \dot{0} \dot{0} \dot{0}$

nin Nin

$0: \because \frac{12}{0}$

i० 100

0
0
0
0
0
0
0
2
2
2
0
0
0
0
0

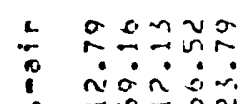

$i$

$\sin 2 \pi=$

Trist

$\dot{i} \dot{i} \underset{i}{i}$

nn

$\sin x \sim a$

2 $25 \div \div \%$

开

$c<c=c$

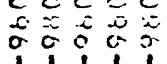

프워요

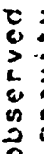

$\dot{x} \dot{x} \dot{0} \dot{0}$

in $c x_{i}$

mrim

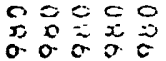

* ニேーேー

E $\cong N \geq=$ $\therefore \dot{0} \dot{0}$

웜ำง

\section{?} $\Rightarrow 0 \sim \approx \tilde{n}$

nmiñ

$7 \div \div \div$

$\stackrel{2}{2}$

$\infty 60 \mathrm{~nm}$ x.

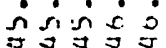

落 テす寻

coococo

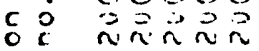

coc so 0

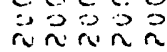

$\because 205 \%=$

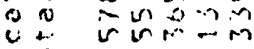

a. $-N u_{1}$

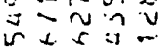

- 1.

$\therefore 5 \therefore \therefore \therefore$

com $=$ in

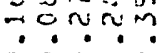

oonno

of 0

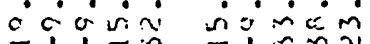

$2000 \approx$

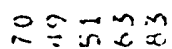

งกำ

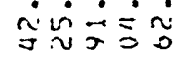

$\approx 0 \hat{a}=$

$\sum \exists \sin$

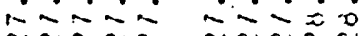

$\tilde{m} \tilde{n} \tilde{n} \tilde{n}$

$==0 \mathrm{c}$

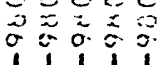

oç⿰彳้อ

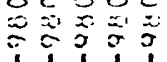

$-050 \mathrm{~m}$

anoruc

$\therefore \dot{\sin } \dot{0}=$

v v

¿V

$\because ? 2 \div \div$

$\therefore m \Omega 00$

$\tilde{\sim} \sim \tilde{v} \stackrel{s}{s}$

$00=00$

nNก0?

$-x-n=$

min

$\ddot{m}=\dot{0} \approx$

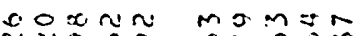
$\sim \operatorname{vis} 0$ نேェ $\dot{\sim} \dot{\sim}$

on 0 ก $\dot{\sim} \dot{n} \dot{n} \dot{m}$

nnm $\infty \cos \infty 0$

$\therefore \dot{0}$

em $0 x=$

$\cong 0 \cdot \alpha x$

iव:

ง. $0=2.5$

is $\dot{0}$

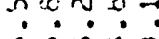

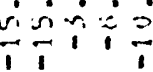$$
\text { (n) }
$$

-

$\ddot{1} \dot{m} \frac{\dot{1}}{1}$

영3ㄱ영

00930

83033

$\therefore \dot{0} \therefore \dot{0}$

वं́⿺辶.

จำ

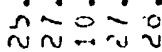

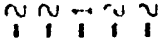

33933

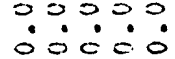

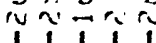

$\exists \approx \cong 00$

穴就文

กิษ

x马mกิ

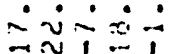

응응

प0:00

옹옹응 ○ं0.

고ำ

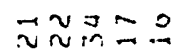

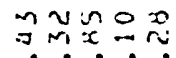

$0-0=0$

$\because \because 30$.

ำำ

00000

oc000

00000

ดูำำ

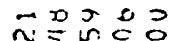

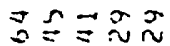

ㅇำ

$\therefore \dot{0}=\dot{0}$

उंin:-

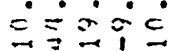

$\approx 0 \sim \bar{\sim}$

iิ

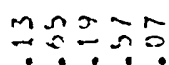

$m \pi n n x$

$\therefore \therefore \dot{0} \dot{0}$

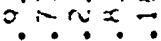

我实部

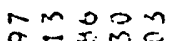

$\sigma \div x=$

$\dot{x} \dot{\sim} \dot{\sim} \tilde{\sim}$

$03=n$

$M=m$

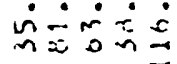

$\because ? \cos$

Nñru

$\exists \infty \simeq 0$ 次

$x=5 \pi$

$\infty \approx \dot{x} \dot{x}$

vinn

$\because n=00$

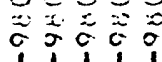

$\dot{0} \dot{0} \dot{0}$

añ

coc $=0$

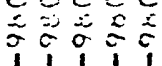

nc n in

$\exists \underset{0}{c}=\simeq$

$\because \dot{0} \div=\dot{0}$

- $\dot{0} \cdot$

- $0=0$

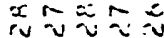

o 000.0

viv

$\begin{array}{llll}\therefore & c & 0 & 0 \\ 0 & 0 & 0 \\ 0 & 0 & 0 & 0\end{array}$

$m=\sim 2$

$\dot{0} \dot{0} \dot{0}=$

$\sim \sim \sim \sim \sim \sim$

=0 $0=0$

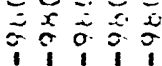

$\approx-x \cdot \ln$

$\because 52 \pi 0$

$\therefore \dot{0}=0$

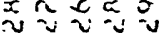

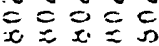

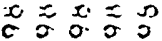

பับ55

넌ㄷㄴ

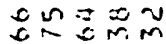

テธับั

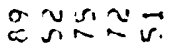

$\dot{0} \dot{0} \dot{\sim}=\dot{0}$

메는

manom

네의

$x \approx \vec{\pi} \approx=$

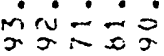

$\exists \therefore x==$ $=2$ $\Rightarrow \dot{x} \dot{0} \dot{0}$ nกñ $00 c=0$ के $2=0$ 1111

$\rightarrow \sim 5 \leq$ $\dot{0} \dot{0} \dot{i}$ $\hat{\sim} \approx \tilde{v} \tilde{v}$ $C=\equiv 0$

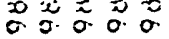

$\div 5550$

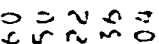
$\because m=\div$ arovim

o。

วกㄴ $\because \sim \hat{n}-$

Thsor

$\sigma=n=0$

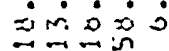

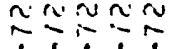

$\dddot{N} \because \because \frac{n}{1}$

$\sim: \equiv x x$

$\dot{\sigma} \dot{\sim} \dot{x} \dot{0}$

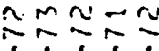

$0 \operatorname{in} \curvearrowleft \vec{n} \Omega$ $\dot{0} \dot{0} \dot{0}=$ - mnNm

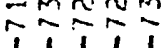

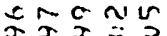
$\therefore: \dot{0}$

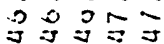

므믐 $\because \therefore \therefore$

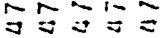

可宁恋

$\vec{J} \vec{J} \ddot{J} \vec{J}$
Mccoc uñ

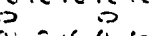

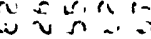
a Nar
$=000 \%$ ñũún UnNAm winn NA.... $;: \therefore$

1. 4. 


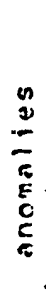

2
3
5
0
$c$
2
$\vdots$
$\vdots$
$\vdots$

is : $0 \div 0$ $\therefore \div \div \div$ in

$-m>-m$

$\sim 0 n n$

$\because \dot{x} \dot{\square} \dot{\sim}$

$\vec{i} \overrightarrow{1} \vec{i}$

20390

$\therefore \therefore \therefore \circ$

00000

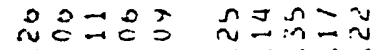

ن

$\therefore \dot{0}=0$

$\therefore \dot{0} \dot{0}$

วกับำ

$n=n 0$.

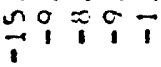

$\because 3 \exists$ ำ

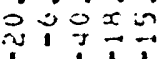

$n n \approx n 0$

$\dot{\sim} \dot{\sim} \dot{\sim} \dot{m}$

$m 0 n \hat{0}$

$\exists \tilde{v} \tilde{v}$

$\therefore \sim c \dot{0}$

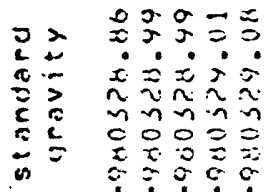

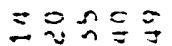

$\dot{x} \dot{2} \dot{2} \dot{x}$

$\approx \sim n n n$

$c=c \equiv 0$

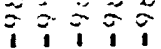

$\because \overrightarrow{0} \approx \vec{n}=$

$\begin{array}{ll}2 & 2 \\ 2 & +1 \\ 2 & = \\ 2 & 2 \\ 2 & 0 \\ 0 & 2 \\ 0 & 0\end{array}$

*

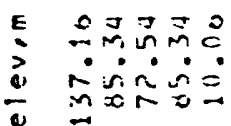

c Nm心in

$n=\geq x$

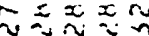

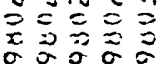

$\because \div \div$

Dㅁำ

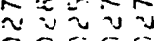

$\begin{array}{llll}0 & 0 \\ 0 & 0 & 0 & 0 \\ 0 & 0 & 0 & 0 \\ 0 & 0 & 0 \\ 0 & 0 & 0\end{array}$

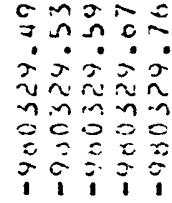

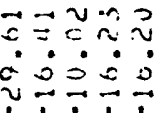

ㄱำ $0 \Omega$

$\therefore=\therefore \therefore m$

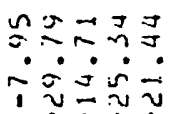

$\approx \approx \because \approx 0$

$\overrightarrow{1} \overrightarrow{1} \overrightarrow{1} \overrightarrow{1}$

$\hat{0} \cdot \hat{\imath} \vec{\sim}=$

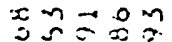

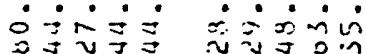

ํํํํํํำ

$\dot{v} \dot{0} \dot{0} \dot{0}$

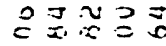

$\therefore \dot{0} \dot{0}$

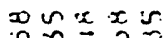

- $: \div$

วง

ปที่าน

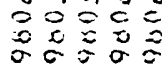

טنu

可舟

o.v? $n \mathrm{~m}$

$n \approx 00 \Omega$

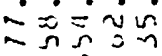

$\overrightarrow{0}=x \geqslant ?$

$\approx \cong \approx \Xi \approx$

$\approx \simeq x \approx \simeq$

c in inim in $\dot{0} \dot{0}$

$\sigma \sim \Omega=m$

$\dddot{n}=\approx \sim ⿻ 上 丨$

$\Rightarrow \dot{c} \dot{\sim} \dot{v}$

$\cong=m \cong n$

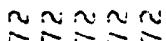

1111

\section{$\underset{x}{x} \tilde{v} \cdot v^{m}$

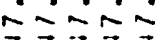 \\ ज्ञज可}

$r--=0$

OMNN

$\because 2 \div \div \div$

- $\dot{0} \cdot$

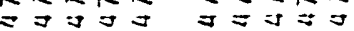

牙究正方

$\ddot{\Xi \vec{J}} \vec{J}$

$\vec{J} \vec{\Xi} \vec{J}$

$=00 \% 5$

กิñ

oceco $\vec{n} \vec{n} \vec{n}$

$\vec{n} \approx \sim \vec{n} 0$

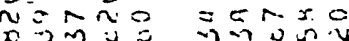

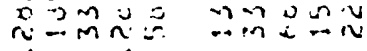

$\because \cdot \because$

$\because \div \div ? \div$

$\dot{j} ; \dot{j} ; \dot{j}$

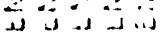

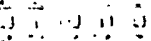

$000 x \pi$ ลกัก

viva

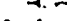

$? \div: 5 ; ?$

A 3 is is

$\because$ iा 1 . $x \in=\pi \sim$

$\therefore m=m \sigma$ $\dot{0} \dot{0}$ $\operatorname{cin}=n \cdot 0$

$\sin x$

$\therefore \dot{\sim} \dot{\sim} \dot{\sim} \dot{\sim}$

Niñ

$0 \hat{0}=0 \hat{0}$

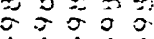

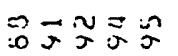

$\dot{2} \dot{2} \dot{2}$

nññn

00000

$0 \approx 00=$

movein

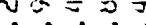

$\ddot{i} \dot{i} \dot{z}$

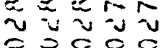

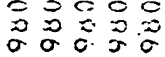

OSTOR

$\div \div \div$

c

v v v v

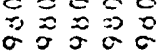

둔ㄷㄴ

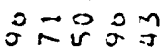

iv $\dot{0} \dot{0}$

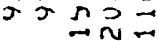

$\nabla \infty n \pi$

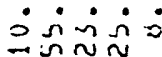

$n-\cdots n \sim$

$1 \% 1 \%$

$\infty \sigma=n \ln$

$\therefore=5: 0$

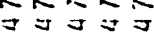

$\vec{\Xi} \vec{J} \vec{ज}$

பับง

o $32 n$

$-0 x \sim N$

$\tilde{\sim} \sim n$

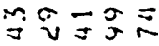
$\dot{i} \dot{\sim} \dot{\sim}$

$-m \sim n \sim$

$\because 1 \div 1$

웡요 $\therefore \therefore \therefore$

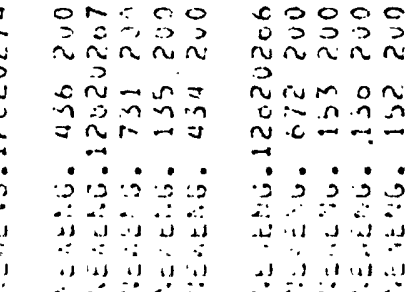

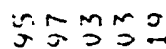
$\dot{2} \dot{0} \dot{0}$

$\sim \tilde{n} \sim n$

$\Leftrightarrow 00=0$

芯芯莎

$\simeq \hat{n} \leq$

- $\dot{0}$

$n=\sim n 0$

$\approx n=\tilde{y} \approx$

$=0 c=0$

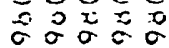

ธ5ธบ

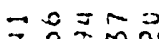

-?

긍ำ

กข $v 00$

- !. .

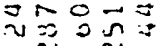

N $\sim 2 x$

$\therefore \dot{0} \dot{0}=\dot{0}$

$\sim \sim \cdots \cdots$

$\because 2=2$

v $\bar{v} \bumpeq \tilde{r}$ ¿0: $\dot{0}$ mn co $=50$ c.

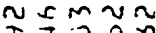
$\therefore \dot{i} \dot{v}$

$\tilde{\sim} \bar{v} \tilde{v}$

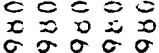

드.

x。นn $\dot{v}: \dot{i} \dot{0}$

mm-m N

$\pi \div \div \frac{1}{1}$

$=m m-$

$m \exists \because n$

$\dot{m j}=$

nnn

$00=0$

c.

$\hat{2} \approx=$

imin

c: $2 \approx$

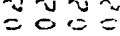

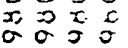

๖๘こう

$0=$

$m=2$

ת $\mathrm{N}=$

ำว

$\therefore-\therefore$

可岗

วอ $\therefore 0=0$

$\because \overrightarrow{9} \overrightarrow{9} \overrightarrow{3}$

$0 \sigma a=$ $\dot{0} \dot{0} \dot{0}$ inำ

ENan

$11 \%$

OORO= $\vec{n} \hat{n} \vec{n}$ $=r$ n

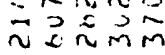
$\because 2393$

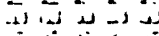
1 i is $n=\ln =0$ Niñ ग? anrum:

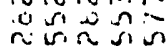
$\because \div$ ; 39

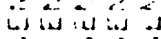
لِّ $m m m x$ $\because \simeq \sim:$ $\dot{0} \dot{0} \dot{0}$

当すすす $\infty 00=$ यก n ? 0 or $n$. $\therefore$ n $\rightarrow-r-a$ $\therefore 25$ is $\because 1 \leq$ $\because+1-1$ 


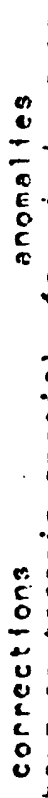

잉ㅇㅇㅇ

- $\dot{0} 0 \dot{0}$

:

- $\approx a \approx n ̃$

a $\dot{0}$.

i. = inn

$\begin{array}{ll}0 & 2 \\ 2 & 2 \\ 0 & 0 \\ 0 & > \\ c & 0 \\ 0 & 2 \\ 0 & 5 \\ 0 & 0\end{array}$

요요

$\dot{D} \dot{D} \dot{D}$

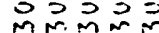

nnn $n$ n

文

o. $0=5 x$

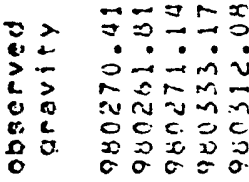

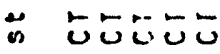

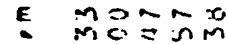

i ncisio

$\div 200$

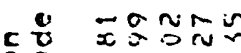

:

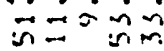

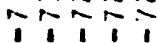

$\exists=0=m$ $\dot{0} \dot{0} \dot{0}$ $\begin{array}{lll}0 & 0 & 0\end{array}$

$\vec{\nabla} \vec{\Xi} \Xi$ $n \approx n \sigma i$

$0 \div \div ?$

गंखि

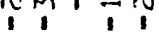

$\approx \sigma \bumpeq 0 m$

$\because \dot{\vec{\sim}}-\dot{v} \dot{0}$

03303

ல் $\dot{0} \dot{0}$

32333 $\therefore \therefore \therefore \circ$

\section{0

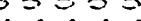
OCं00

32393 ¿००००

$39=30$ ¿ $\dot{0} \dot{0} 0$

03009 ¿0000

$x \simeq 2 \pi$ $\therefore \div \div-\div$

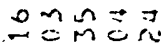
$0 \dot{0}=\dot{0}$

$\dot{0} \dot{0}=0$

믄른 $\therefore \circ \dot{0} 0 \circ$

$\sim n \rightarrow 0 \Omega$

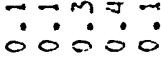

ดขกั ¿نं

긍ำ $\therefore \dot{0}=0$

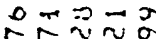
$n=\frac{1}{m}=$

$\equiv \underline{2} \div \frac{n}{2}$ $\therefore \therefore \therefore$ $\div 1 \div 1 \div$

$x=00$

$\dot{\sim} \dot{\sim} \dot{m} \dot{0}$

ข้ บ

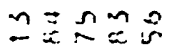

$\dot{0} \dot{0} \dot{0} \dot{0}$

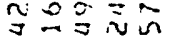

$n: \exists=n$ - $\dot{i} \dot{i} \dot{0}$ บ $\vec{n} 0 \mathrm{~m} \cong \stackrel{0}{0}$ -cin

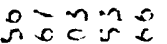

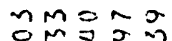
$\therefore$ 的远 $\Omega=0 \div 5$

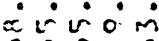
$n=n$

ज๘

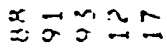
$p=j-\dot{m}$ nnnm

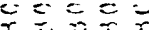

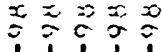
$m a n$ mas m m nrinn $n$ $50 \equiv 5$ c $\approx \approx 000$

然 $\dot{2} \therefore \dot{0}=$

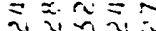
ข้นี้:

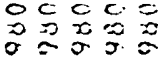

$\stackrel{5}{5}=5$ $\dot{0} \dot{0} \dot{0}$ $\because 0=m \bar{v}$ $=c 0 c 0$

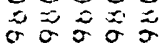

๖テテらら

$\cong \overrightarrow{2}=0$

ว. $m \therefore=c \approx$

는

유를

$\therefore \dot{0} \dot{0}$

$\Rightarrow \begin{array}{lll}0 & = & 0\end{array}$

$\sim c-0$

$m \approx \equiv \equiv N$ $\vec{m} \bar{\sim} \bar{n} \vec{m}$ mnnn

$E \bar{x}$ $\approx 200$

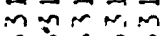
n่ $v_{1} n$

$\exists \equiv \equiv \equiv$ $\approx 0 ; 0$

$\bar{n} \pi \cong=$

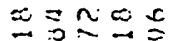

०20 : 0

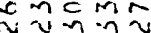
vin $\begin{array}{llll}c & 0 & 0 & 0 \\ \sigma & 0 & 0 & 0\end{array}$

- var $\bar{v} \approx \sim ⿻ 上 丨$ $c=0$

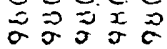

둔

テேั50

고ำ

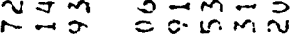

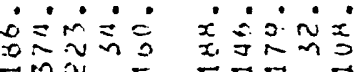

$m \Sigma \Sigma=n$ $\therefore \therefore \therefore \dot{i}$

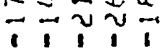

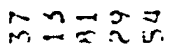

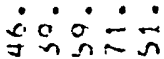

$\vec{n} n=\ddot{n} n$ ขึก

$\Rightarrow 0 \hat{n}$ irini añ ज经 $\dot{x} \dot{0} \dot{0}$

$\sin \frac{5}{2}$ $\dot{0} \dot{0}=0$ $n n n m$

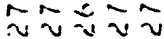

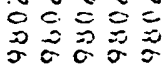

ㄴํㄴ

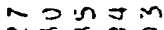
0 in

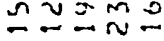

nesino

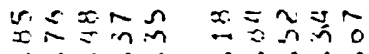

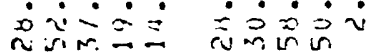
manr N⿴囗n $1 \div 1.11$ 11111

$m$ mi: $=2$

mm SNO $0 \dot{0} \dot{0}=\dot{0}$

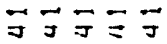
$\therefore \dot{0} \dot{0}$

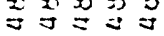

テすテす

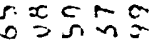
$\dot{u} \dot{\vec{r}} \dot{\vec{z}} \dot{=}$ nMm N

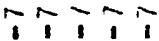

orinon $\rightarrow x 300$ $\dot{x}=\dot{0} \approx \dot{0}$

ज可 $\vec{\nabla}$

$M=c o n$ ar

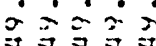

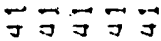

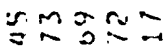
$\dot{j} \dot{0} \dot{0} \dot{0}$

$n-\sim n \sim$

$\div \div \div \div$

$n m a n$ $\therefore \dot{0} \therefore \circ$ $\sigma=0$

可宁宁 $\approx m \cong ั 55$ $\ddot{n} \approx \dot{v} v$

$0=0$

$\approx 0$ $11 \% 1$

ำกำ $\approx \approx$ $\dot{\sim} \dot{v} \dot{x} \dot{v}$ 0

$\because \therefore \approx \approx \sim ⿻ 上 丨$ $\dot{v} \sim \tilde{v} \sim$ $m \backsim n n$ 三 $\approx \approx 0$ व

음둥 i $\dot{v} \dot{0} \dot{-}$

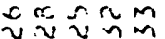
vṼ๊ $\begin{array}{llll} & \\ 0 & x & 0 & 0 \\ 0 & 0 & 0 \\ 0 & 0 & 0\end{array}$

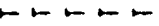
$\sigma n m n \rightarrow$

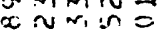
向

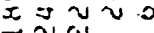

을ำ rimis $\stackrel{m}{\sim}-\underset{0}{ }=0$

c. $\dot{\sim} \dot{0} \dot{\vec{O}} \dot{0}$

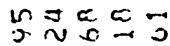
ñmiñ nn $-n$

$n m-? N$ ㄷำ

1151

a $\rightarrow \ln \infty$ $\therefore \dot{0} \div$

$0 \sqrt{0} \overrightarrow{5} \hat{0}$

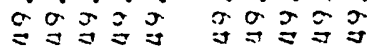

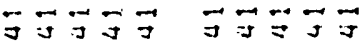

$c=c: 0$ ñun or-

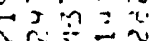
$\div \div 3$ $\div+.41$ $n=000$.

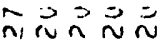
$\vec{n} \pi \sim \alpha$ $\approx$ 浮 $\approx$

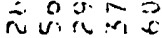
$\because .09$ $\therefore=03$ $\therefore$ 两
웅ㅇㅇ $\ddot{n} \dot{n} \ddot{n}$ $\Rightarrow+c \tilde{n}$ J可 - $\because$ $\because \because 92$ is in 

$\because \cdots \div 0$ ก 六的品

amn

กำ

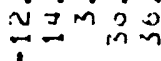

- 99330

$\ddot{y} \dot{0} 000$

焉



ํำวn $\because \min : ?$ $\therefore 0000$

กำ

$\dot{=} \dot{0} \dot{0} \dot{m}$

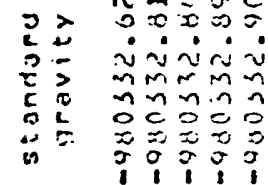

$\Xi \bar{v} \stackrel{x}{x}$

a

可牙行

$\therefore=00$

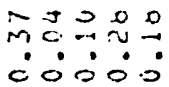

여영ㅇ

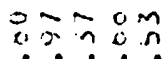

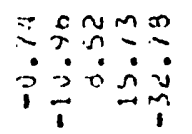

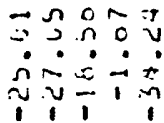

in

$\because \frac{1}{1} \geq \frac{j}{i}$

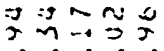

จำำ

중ำ

$\ddot{\sim} \ddot{\sim} \dot{\sim}$

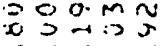

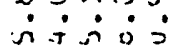

$\sin \sin =$

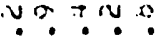

$\dot{\sim} \dot{\sim} \dot{v}$

1111

는

$\because i \dot{i}=$

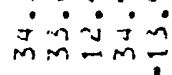

$\dot{0}: \dot{j} \underset{i}{0}$

守守方立

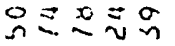

ooomn

ن $\overrightarrow{3} \dot{\mathrm{s}}$

orsmin

a:

in $\div:$

in

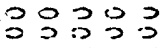

$223333 \cong 3398$

33000

00000 $\therefore \leq 00000000$

$00000^{\circ}$

$\dot{0} \dot{0} \div \dot{0} 0$

$\therefore \dot{0} \dot{0}:$

$3 ? ?$ $\therefore<0=$

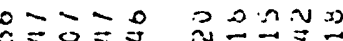

$\partial m \approx 20$

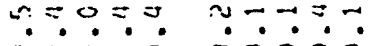
$\because \because \div \div$

in

$\vec{\sim}$ iñ $\overrightarrow{0}$

$P \equiv n \leq n v$ वं0்

na?ำ? 00000

$\approx \approx 0 \approx \approx ?$

$0 \pi m \simeq \vec{v}$

$\vec{\exists} \vec{m} \hat{0}:=$

กำ 20

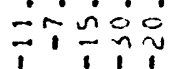

nंजिं

invo:

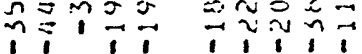

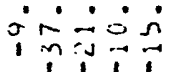

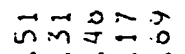

$\approx=0 \hat{n} \vec{m}$ $\therefore \dot{0} \dot{0}$ $\operatorname{in} \hat{i} \bar{v}$

$\dot{0} \dot{0} \dot{0}$ $\because \because \frac{1}{1}$

$\cos =$ विं

"

ㅋํํำ

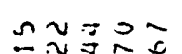

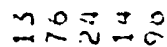

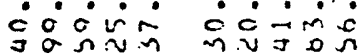

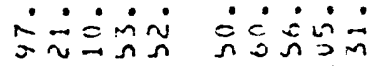

$\sim \sin \sim \sim \Omega$

-0.0m $=$

\section{a}

น ก บ

$\dot{x} \dot{0} \dot{0} \dot{0}$

ํํㄹㅇㅛ

$\dot{x} \dot{0} \dot{0}$

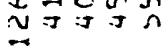

จูบำะ

ำำ는

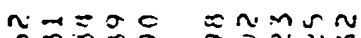

$\sim F \sim m n$

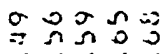

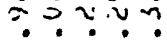

$\because ?$ ?

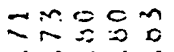

ทีติnin

mกnn

o: $0: 3$

ตีที丶万仒

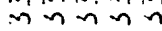

ใิำ $=$

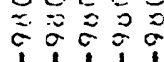

$\therefore \dot{n} \dot{n} \dot{x}$

minn

0
0
0

जै:

ज和的

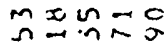

o $00=0$

过是品:

va

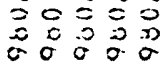

กำป

$\dot{0} \dot{0} \dot{0}$

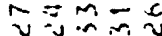

3053

करें

is $s \hat{v} \stackrel{0}{v}$

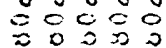

0。丁n

$m \leq \sim \approx \sigma$ $n n n n n$ nnn $n$ n coocc

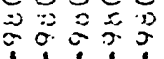

vinivis. Mm

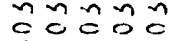

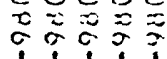

o ヒேヒே

๖ここここ

ニニーニ

\section{ேㅜ}

nim-ro i i i

vo

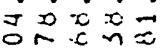

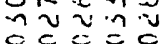
定 0

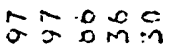

sisin

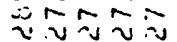

$c \equiv$

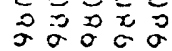

3200 可可可

ทnทin

oै 000

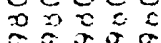

i

유마 $\vec{n}$

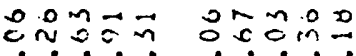

$\bar{m} \approx \dot{\tilde{n}} \tilde{v}$

의의

모ำ

こ只只二

๖ここここ

$x \rightarrow 50$

$\therefore \dot{0} \dot{0}:$

ขิง

ปินี้

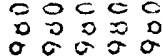

$\approx \hat{n} \ln \leqslant \hat{\infty}$

$\dot{\Sigma} \dot{m} \dot{m} \dot{\Sigma}=$

บำ.

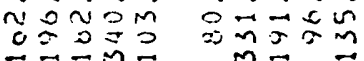

$\approx=0$

$\dot{0} \dot{0}$

- uㅣㄴ

ธேบับับ

$\exists=0 \rightarrow \sim$

$\therefore \dot{0} \therefore \dot{0}$

onin

ป $0 m-$

$\therefore \cdot \dot{0} \cdot$

$\ddot{\sim} \vec{\sim} \vec{\sim}$

nn n.

이잉

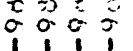

$\sum_{0}^{c} \ddot{n} x$

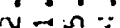

$x$ w $2=$

$\widetilde{c} \tilde{c}=$

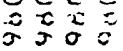

$-25-2$

$\hat{\sim}$ ํำ

$\dot{m} \dot{m}=\dot{1}$

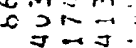

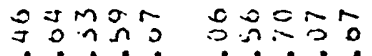

c⿻ำ

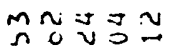

m的云品

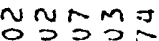

onison $\dot{0} \dot{0} \dot{\sim} \dot{\sim}$

จ $200 \pi$

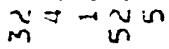

$\ddot{\sim}-\dot{n} \dot{\sim} \dot{v}$

NUNM- NMNNR

n $\sim m \sim$

NMNMN NMNMN

$m m \sim m n$

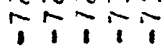

$111 \%$

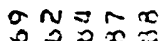

$m \approx c-0$

กี⿻

$\sim m x x 0$

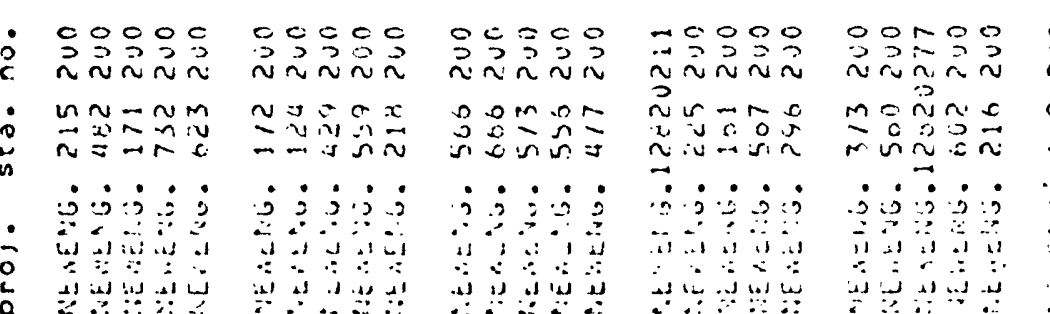

$m \exists r a m$

- $\cdot 0$

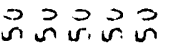

可宁苛

$\dot{0} \dot{0} \dot{\sim} \dot{n}$

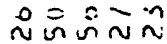

$m-m \sim N$

$1 \div \div \%$

의므

$\therefore ?$

นึ่ง

-m-m?

$\div \div 1 \%$

men ema

$0.02 \pi$

$\therefore \therefore \dot{0}$

An

ij $\dot{0}$

$\vec{\exists} \ddot{\vec{J}} \vec{J}$

$\because \vec{J} \vec{J} \because$

$c=000$ जेंगें

s.

(

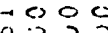
NnN $\sim m-n$ J n.M...

$5=5$ 


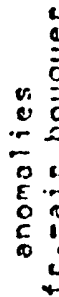

ㄷ. 응으을

은.

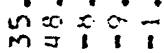

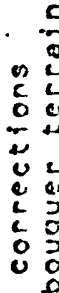

$m x a z$ ¿व்0ं

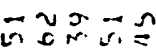
$\therefore \dot{\therefore} \dot{\sim}$

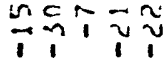

mบูกั $\because \because \because \because$

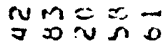

으요

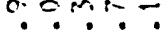
sis.

$m=050$

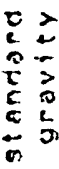

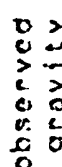

CNM = M 독 OC = Co $\begin{array}{ll}x & 0 \\ 0 & 0\end{array}$

영영요 ¿0ं0ं

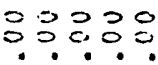
$=000$

Nํ류 $\therefore 000$

siman

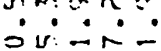
$\because \div \div \overline{1}$

กขกภอ $\because \because ?$ ?

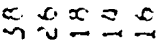
$11 \div 1$

$\vec{C} \overrightarrow{0}=0$ $\dot{0}=$

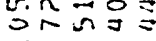

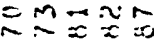
Jं $\dot{\vec{B}} \dot{\mathrm{B}}$ $\min \approx \sim$ ติน coc coc 1
0
0

$\exists \therefore m_{1}=$ $\dot{j} \dot{n} \dot{n}$ mmmm ทีทั่อ

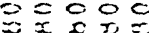

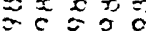
is:

50500

o. 0 a $\dot{\dot{v}} \dot{\vec{q}} \dot{\vec{r}}$ $\approx \tilde{\sim}=\tilde{m}$ चñm

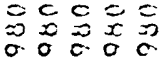

唡 m $0=0=0$

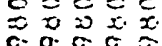

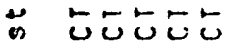

こここここ

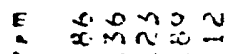

\& mosis

$\because \cong \bar{m}$

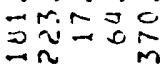

5
0
0
0
0
0
0
0

is 005

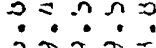

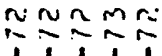

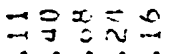
$\stackrel{\sim}{\sim} \dot{0}=\dot{c}$

NMIn $\frac{1}{2}=12$

$\because x \approx n ?$ $20 D j$ ข v vis

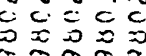

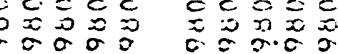

ここேこே

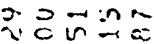
visiom

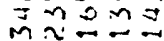

$m=m o$ $\dot{0} \dot{\mathbf{i}} \dot{\mathrm{i}} \dot{0}$

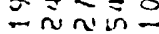
m vañ $\div \div \div \div$

$M=\backsim n$

consis

orom

$\because \simeq n m$ 向殏立 जis is sis

すコすきき $\dot{a} \dot{0} \dot{1} \dot{1}$

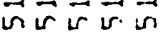

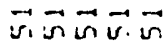
すコすテす

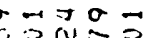
$\dot{n} \dot{0} \dot{0}:$ -rivi:

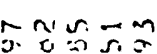
$\therefore \sin ^{\circ}$ กิंजि

룽응ㅇㅇㅇ ¿0ं் $\therefore \therefore 0.0$

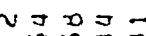
$\because \pi+2=$ Oc 500 1ิ1\%

证引气 ñmin: นתลn:

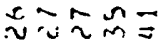
$\dot{n} \dot{s} \dot{0}$ ññm ตำ

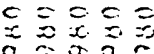
i

$0 \approx 4000$ $\dot{n} \dot{i} \dot{0}$ vivuv

こここうう $\bar{m} \Xi 2 \Xi=$ Doñ 개에

요응 $\dot{\sigma} \dot{\infty} \dot{\infty} \dot{\infty}$

Namn 1.T.

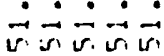
すすすすす $\therefore \circ \therefore$ in minn 证实云=
39893 ¿0ं $0^{\circ}$

ํำ约的

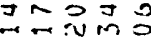
$\therefore \dot{0} \dot{0}:$

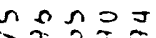
$\because \therefore 3 \approx 2$ s.civs: 1?

$020-1$ $x=0 \approx$

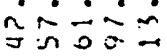

$0 \approx 000 \quad$ cxm心兄 ṽdin

$\cong \cong \Xi ิ$

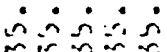
min $m v_{1}=0$

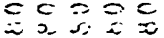
\% $15 i 9$ $\dot{\sim} \dot{v} \dot{x} \dot{\sim}$

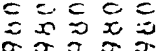

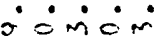

sis: s.

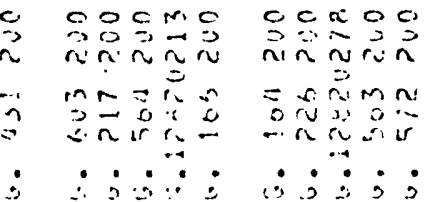

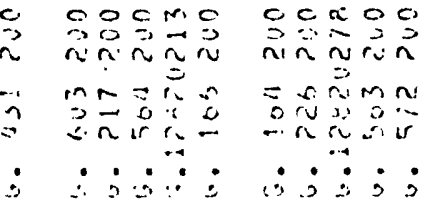

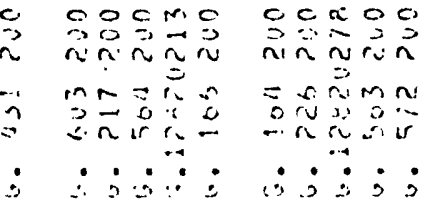

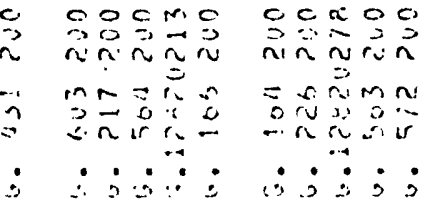

$0=0 \mathrm{mos}$ $\therefore \dot{0} \dot{0}$ ññ

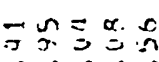
$\therefore \dot{0} \dot{0} \dot{0}$

$n \simeq \tilde{z} \tilde{x}:$

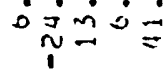

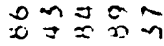
ज㐫市市

32333 $3=? 3$ 0000

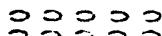
․․․․․ $\therefore 000$

$000=0$ 군? 00000

กงิน

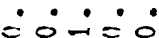

nno n

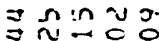
0ं0ं0 व०0:i

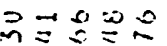
$\therefore \dot{0} \dot{0} \dot{\sim}$ $\rightarrow \because \dddot{i}$

$\tilde{n} \approx \cdots$ ris $\sin 5 \hat{5} 35$ 郝司方 $\therefore \dot{0} \dot{0}=$ $\approx \sim 1$.

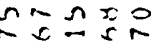

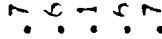

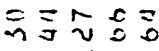

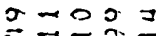

$\vec{A}=\bar{\Omega} 2$ $\because \because \because$.

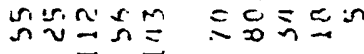

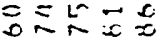
$\dot{\mathrm{n}} \dot{\mathrm{n}} \dot{\mathrm{n}}$ nmm $c=00$

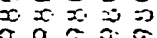
1 $91 \%$

$=\pi=\ln 0$ $\because \div 3 ?$

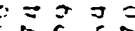
ง v v

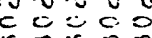

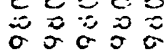

$x \approx \sin$ $\dot{0} \dot{0} \dot{0} \dot{0}$ minn ㄱำ

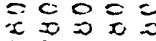
$\begin{array}{llll}1 & 1 & 1 & 1 \\ 1 & 0 & 0\end{array}$

$\nabla c \cdot \sigma \approx=$

$\eta x^{2} \cdot x$

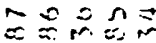

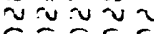

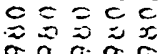

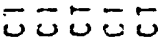

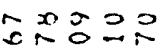

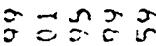
$\dot{x} \dot{0} \therefore \dot{0}$ 过正 - $\dot{3} \dot{0}$ $\exists \vec{\sim} \tilde{D}$

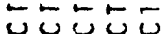

ชิ

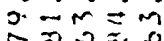

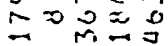

苾品三ニ

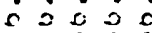

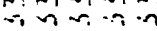

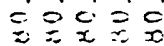
ज行

omno $: \because ? ?$. วอบ 원 $c 00 \equiv$

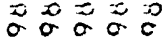

七5ここ ?onn $\because 000 m$ $\dot{x} \dot{0} \dot{x}$ $\approx 0-n$ -

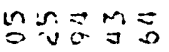
$\dot{\sim} \dot{\sim} \dot{\sim} \dot{0}$ กN $\therefore-1-1$

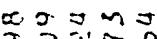
$\therefore \dot{0} \div \dot{0}$ Sin in

$\Xi=M N \cong$

MO $=$ $\because 90$

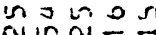

u-m $v m$ 준도

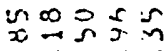
त $\overrightarrow{0} \tilde{m}$ m.m-cn ริกิริ

ภิsin:

on

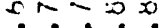


ก ก

$0 \sin 0=$

$\dot{0} \dot{0} \dot{v} \dot{v}$

$1 \div \div$

동요

宁婇

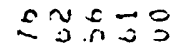

$\because 3 \div 3$

$\because \because \frac{1}{1} \overrightarrow{1}$

กูำงภำ

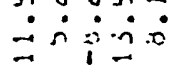

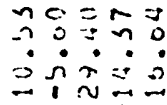

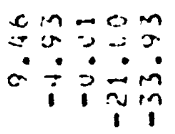

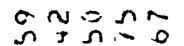

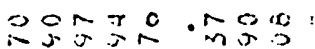

$\dot{0} \dot{0} \dot{0}$

$1 \div$

ลลำลำ

Non $0 \approx$

$m \approx \sim x \sigma$

푸요

$\dot{i} \dot{i} \dot{i}$

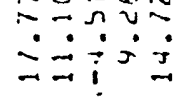

ثُ

$\therefore \therefore \dot{0}$

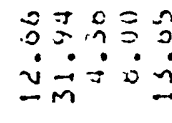

응?

웅옹응

32303

3여영

¿0ं0.

00500

$\dot{0} \dot{0} \dot{0}$

옹공영

0ं0்0

○ं0.

33398 $\therefore \dot{0} \dot{0} 0$

35333

$\therefore \dot{0} 00^{\circ}$

9330

0ं0ं

n马ת

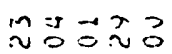

กิจภิษ

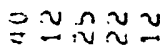

בㅇํㅇำ

¿ंव்.

वंड: $=$

विंक्ष

$\therefore$ ㄷㅇㅇㅇㅇ

वंi०

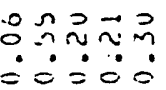

$=0200 \%$

iं00் $0 \dot{0}$

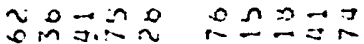

กำ:

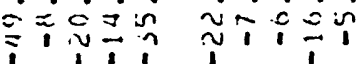

$\dot{i n i m i c}$

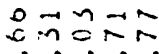

$\therefore \overrightarrow{0 \Omega 0}$

जिंنे

१1

iि $1 \div \div$

ธิํํำ

$\dot{i} \dot{i} \dot{x}$

$\because \div \frac{\pi}{1} \div$

$\exists \exists \cong n$

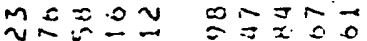

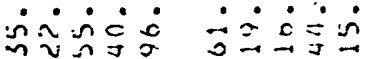

$\approx 0=20$

$\overrightarrow{3} \cong ㅇ ㅛ ㅇ$

$\therefore x \equiv x \overrightarrow{0}$

ivं $\dot{0} \dot{0}$

$\dot{x} \dot{x} \dot{i} \dot{-1}$

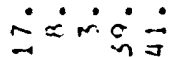

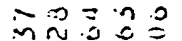

$\therefore x=5$

$m=\pi \sim r \quad n=\approx m 0$

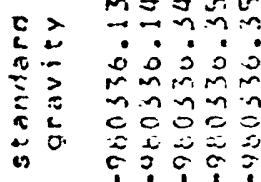

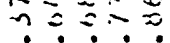

$\dot{0} \dot{0} \dot{0} \dot{0}$

ทnทnn

年

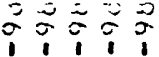

$\because \approx \Omega \approx \Omega$

$\hat{2}=0$

$\dot{0} 0$

0 is

$n$ n.m

$\therefore \equiv 202$

$=\overline{1}=5$

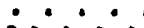

mnmm

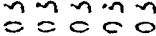

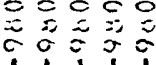

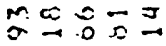

$=00$ 年

$\therefore m=0$

c. $-x \times n$

¿a

$\dot{\nabla} \dot{=} \dot{0} \dot{0}$

$\sum \bar{i} \approx \tilde{v}$

$\begin{array}{lll}0 & 0 \\ 0 & 0 & 0 \\ 0 & 0 & 0 \\ 0 & 0 & 0\end{array}$

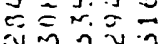

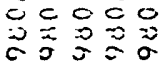

$\therefore \dot{v} \dot{0} \dot{0}$

$m \sim v m$

ñn

等

$\sin$

$\dot{0} \dot{0}=\dot{0}$

च $v=0$

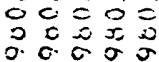

\section{1}

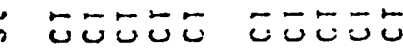

こここご

ここここう

$00 \geq 0$ 02000

$\vec{n} \vec{m} \approx v$

$\begin{aligned} 2 \\ 0\end{aligned}=\infty$

m?

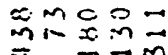

- mai

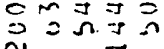

$\hat{n} \dot{\sim} \dot{0}$

กิ่舫

กำ:

$n \nabla n$

กำำ $\underline{i} \dot{\sim} \dot{\sim} \dot{\sim}$

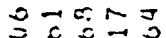
i் $\dot{\sim} \dot{\vec{I}} \dot{\mathrm{I}}$

풍요궁 $\dot{-\dot{a}} \dot{v}$

ก

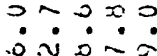
$\Xi \dot{m} \sim a$

$\because \because 2 . \bar{n}$

น $\sim \sim \sim ⿻$

NMn

$\dddot{m} \simeq \frac{N}{1} \div \frac{m}{1}$

$\sim \sim \sim \sim N$

$1 \div 1 \%$

$\div 11 \%$

$\div \div \div \div$

$\because \because 0 m$

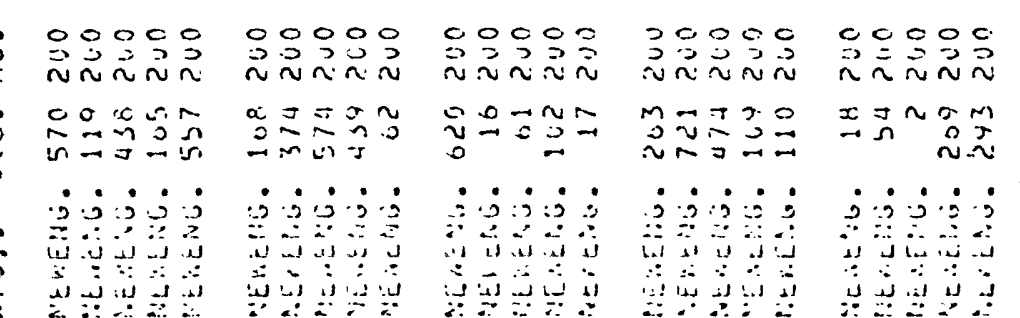

$\sim 0 x=0$

$\because \because \div$

$n m m$ m

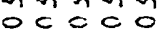

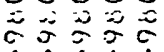

$\alpha=m O N$

$\therefore \therefore \dot{0}$

$n \approx n=0$

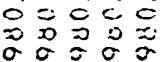

$m M 20$

nin

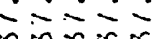

$n n n n n$

$0=c=0$

$\approx \approx \approx \sigma \tilde{\sigma}$

111

r $\sigma \geq 0 m$

í $\dot{0} \dot{-1}$

$\sim 0 x=$

$30=0=$

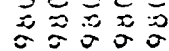

こここここ

순

$\rightarrow: 0 \stackrel{0}{\sim}$

든ำ

जiñ iv

$\sim \sim \sim \sim-$

$\because \frac{1}{1} \div \frac{1}{1}$

ㄷㄴㄴ

$\Rightarrow g a z \cdot n$

$\because \approx-1$

$x=0 \div$

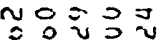
$\stackrel{i}{\sim} \dot{\sim} \dot{\sim} \dot{\sim}$

N $\mathrm{N}$ a

$\because 11 \%$

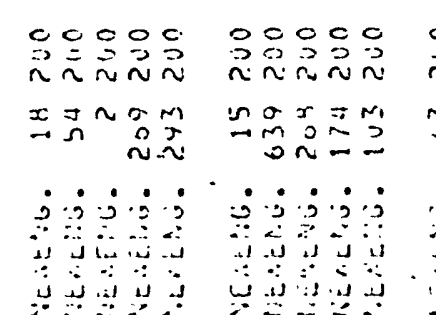

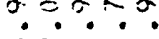

$\div \cong \approx n \overline{7}$

111

อภnภ。

ำ.

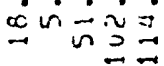

$=\sigma \sim \sigma n$

: $\div$.

innm $n=$

n.nnn

$=00.20$

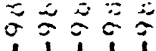

$c \wedge m \subset \Omega$

sn

in $\dot{j} \dot{0}$

in wh

co: 0

$\begin{array}{llll}0 & 0 & 0 & 0\end{array}$

-r

ocin

ก:? ? ?

อㄷำ

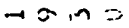
马? ? $\begin{aligned} & \infty \\ & \infty\end{aligned}-\dot{1}=$

$\because n \equiv 2$

$\dot{0} \dot{x} \dot{x} \dot{\sim}$

กำ

$\because \because$

in

nกn

coc

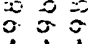

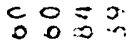

$\dot{1} \dot{0} \dot{0}$

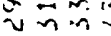

응

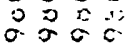

-5:

- $2 n: n$

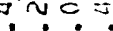

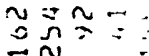

$x=2$

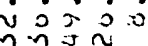

$\sim \sim-M m$

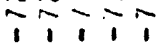

$\dot{0}$ i $\dot{0}$

- in

nก $\sim$ ก

$1 \div 1$

5070

$\because 0=5$

$\dot{n} \dot{n} \dot{n} \dot{n}$

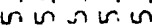

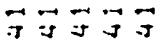

$\dot{m i n} \dot{n}$

nins

学寻苛宁。

0
0
$\vdots$
$\vdots$
$\vdots$
$\vdots$
$\vdots$

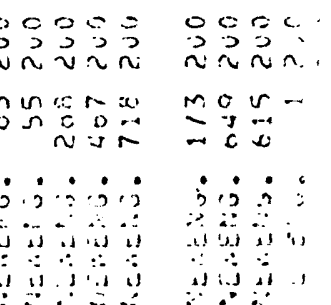




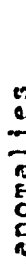

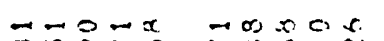

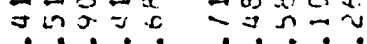
$\dot{\sim} \dot{0} \dot{j} \dot{i} \dot{i} \dot{j}$ $\because 1 \div$

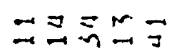

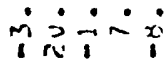
$\overline{1}$

$0 .-c m x$ onv

$0033=$ 0ं0ं

$3 \geqq 930$ نं0்:

$\vec{\sigma} \hat{\sim} \overrightarrow{0}$

定

$80 n=8$

o:

苍

i.

08

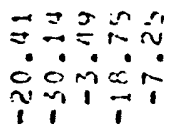

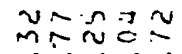
sin $x$

$x_{0 \rightarrow 0}=2$

criss

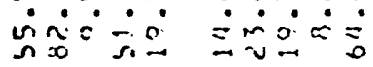
$\therefore$

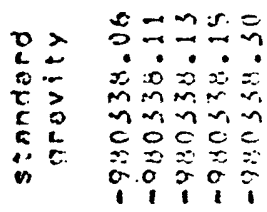

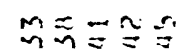
$\dot{x} \dot{x} \dot{0} \dot{x}$ $m \sim n \sim m$ $c=0$ cico

ำ ㄴำำ

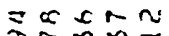

is

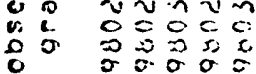

\& ธัษうこ

E 코요뭉

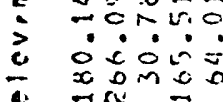
$\dot{=} \dot{0} \dot{i} \dot{0}$ min

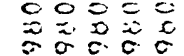

\section{ここここた} $\exists ข \cdots \simeq ?$ $\because \div x=$ ำ范宫

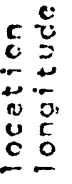

No: 0 ir $\dot{n} \dot{m} \dot{n} \dot{0}$ त̛̣miñ

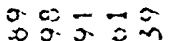
$\therefore \therefore \dot{0} \dot{x}$

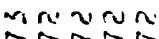
$17 \div \div$ Naran $\frac{2}{1} \div \frac{2}{1}$

c) Nルn ?.? in in wivis

$=m$ us $x$ 武可可

$\sin \Omega=8$ insing

可可 aำ木 $\therefore \circ \dot{0}=$ 彳in 我是的包 $\dot{\sigma} \dot{0} \underset{\sim}{*}$

30339 ¿̊0ं0

อรที ㄷ․․ ic000

m̃s

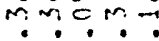
-0ं:

코융ำ - io io

manno $\div 3 \infty=\infty$ ำ

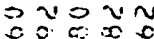
inin 的的兵

$=-1-n$ ดง กภก $\dot{x} \dot{0} \dot{0}$ ring in $c 0=0$ 10

무요 $\because 90$ ini

$\vec{\sim}=0$ mifin: 行?

$\hat{N_{1}} \cong \approx=$

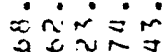

$\vec{N} \vec{n} \vec{x} \stackrel{n}{=}$ $\therefore \dot{\sim} \dot{0} \dot{0}$

$x_{0} \approx \vec{x} \vec{x}$ $\dot{x} \dot{0} \dot{x} \dot{0}$ nn $n$ nn $\hat{c}=0$

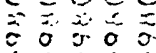

x. $\approx$ บ $\dot{3} \dot{n} \dot{n}$ जiñ ที่

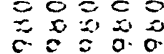

ここここす $\Rightarrow$ in $\because 2$ $x: \because 5$

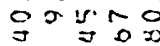

屁足

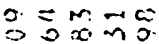
这棉官: N NMMM $\div \div \div \div$ 65050 $\dot{m} \dot{n} \dot{s} \dot{x}$

ton-ron $\because \div \div 0$

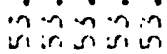

$\square=20$ $0 \approx 5 \%$ $\dot{0} \dot{0}=\dot{0}$ minn $0=0=0$ 过

ヘニ过

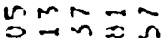

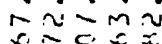
$\sim v \sim \bar{v}$

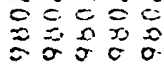

๒

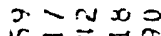
$\therefore \therefore \because \therefore$

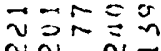
$\dot{0} \dot{0} \dot{0}$

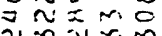
चn ô

ヒัேこち

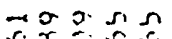
$x x \leq$ $\approx \operatorname{mb}$

的券 $\dot{m} \dot{\therefore} \dot{x} \dot{0} \dot{0}$ ru $-\sim v-$ $\uparrow \div \div \div$

$\infty x x_{0}^{\infty} \approx$

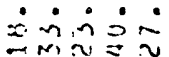
moneres $7 \div \div \div$

ขัน $x=0 \% \sigma$

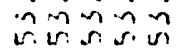

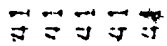

テษ

浔守学宇

on Nmin $3 ? \geq=?$ 穴安安 minnun $\because \mathrm{c}=\mathrm{c} c$

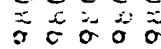

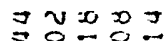
○ं0.

$\because n=0$ $\because 03 \because \div$ $\therefore 000$

$\stackrel{0}{20} \vec{\sim} \approx$ $\because \because 00$

$2 \sim 0 \sim 0$ $\therefore \therefore$ ษิษ

OS- UN $0200=$

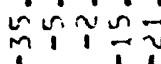
$\overrightarrow{200}=5$

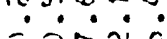

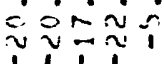

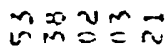

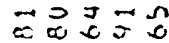
$\bar{\sim}=0 x \overline{=}$ i̊ㅎㅁ

जिएं日 $\therefore$ तix $\dot{0}=$ i 1911

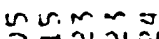
ว.บบบ 可的可 กทักท $c=0$

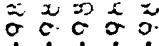

ثิنับ

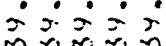
ทnッn $c=c c=$

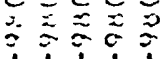

$\stackrel{\infty}{\sim} \approx \mathscr{m}=$ $\dot{a} \dot{j} \dot{z}=$

$\sim \sim x$ s.c 우ㅇㅛㅛ $\stackrel{ \pm}{2} \approx \approx \sigma$

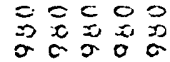
$\dot{0} \dot{0} \dot{0}$ 的的要

$c \circ \equiv=0$

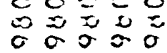

$\dot{\square} \dot{\therefore} \dot{二}$ 割证

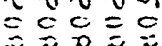
こテこここ

らここここ $\approx 50$

in $x$ in $0=$

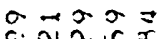

こここここ

$4 \therefore \because \div$

$\stackrel{\sim}{0} \cong 0$

-

$\because \therefore 0 x=$

舟西要

$\dot{x}-\dot{0}=$

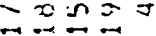

今.

NAOMO

$\beth \simeq x^{x}$ $\dot{0} \dot{0} \dot{n} \dot{n}$ $>200=3$ $\dot{\vec{\sim}} \dot{0} \dot{\sim}$ iñn

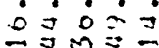
mñ 2 $\sim \pi \sim \sim \sim$ กิรง กิง ษิง

us son

a. $\ln 00=$

NAM $=0$ ir. $\Rightarrow \because \div$ 证的可 - $\cdot \dot{0} \cdot$

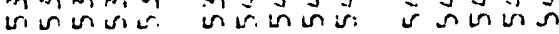

すコすテす

Эすすすす

すすすすすコす

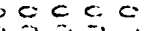

c. 5000

0005 方兄主主

8085 nis añ

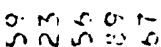
niñ cis 号战嘴

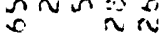
$\underline{2} 2 m x_{i} \bar{v}$ $\therefore \therefore \dot{3}$ $\sim n \cdots n$ 


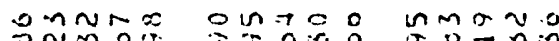
$\dot{0} \dot{0} \dot{0} \dot{0}$ $\dot{v} \dot{\sigma} \dot{\sigma}$ $\because$

$\bar{\pi}$

ह

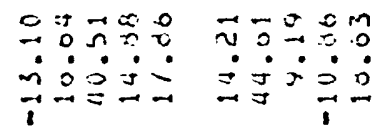

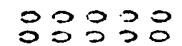

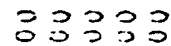

¿ं0்:

$\dot{0} \dot{0} \dot{0}$ \begin{tabular}{ll}
0 \\
\hdashline \\
\hdashline
\end{tabular}

$\approx \approx 3 \vec{v} \approx$

यंग्र

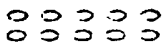

○ंல்:

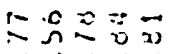

$\hat{\sim} \div \dot{\sim} \dot{\vec{n}}$

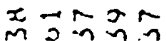

نी

응ㄱㅇㅇㅇㅇ ○ंல்: $\sigma=n n \dot{y}$.

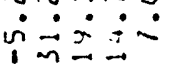

$\mathfrak{1} \div \frac{1}{1}$

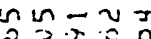

ì نं

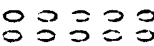

$\dot{0} \dot{0} \dot{0}$ $\operatorname{2n} v \sin$

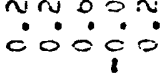

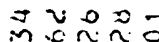

$\because n n ?$

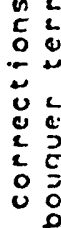

$2 \rightarrow n-\pi 0$

$a \approx \therefore \approx ?$

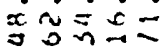

$\therefore$

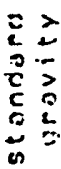

$n \cap n=\approx$ $\dot{0} \dot{0} \dot{0}$

ming

กินทีก

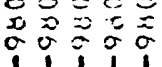

구

$2: \therefore \dot{0} \dot{0}$

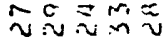

告

$\infty$

๖ธธธย

E 윰ํํำ

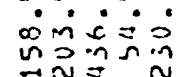

5
0
0
0
0
0
0
0
0

No응

$\because \div$ $\dot{\sim} \dot{j} \dot{\Xi} \dot{q}$

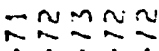

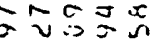

$\dot{0} \dot{0} \dot{z}$

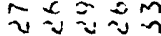

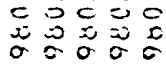

$\therefore n: 0 n$

$\therefore \therefore \dot{\sim} \dot{\sigma}$

$\approx m x \infty$

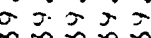

กททณท

$x \approx x=0$

1111

ーேーே

นำำ $m \approx * \Omega$ $\simeq \approx N 00$

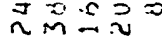

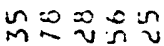
它完完

ヘMミM

$n m 00$
0000
000

고으의

ํㅗำำ

으으요

$\therefore 0-00$

i.

$3 \pi n \pi n$ $\because \div \therefore m$

viv $v 1$

$=0$ ir.

$\because \approx \dot{*} \div \dot{0}$

$\therefore \geq \dot{0} 0$

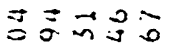

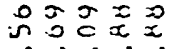

政舟

iिं

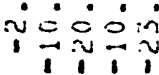

$=\because \because \overrightarrow{0}$

$\because \because \dot{0} \dot{0} 0$

$\approx \rightarrow 0 \sim v$

$\because 0=\div$

$\therefore \min x$

푸

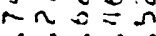

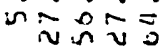

$\therefore \rightarrow x \Rightarrow$

$\because \stackrel{2}{\approx} \approx$

$\because \because \cdots$

会示示

ำ

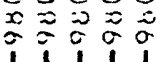

$\wedge \pi \approx 0 \approx$ ?

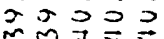
nnn n =0 000

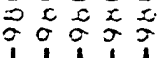

$m=-m o$

n $52 \div$

$\therefore \dot{v} \dot{0}$

c $气$ v v

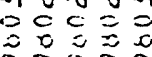

mm

$\because 0 \cong \cong \vec{c}$

ที่ั่ง

ह5:

000

$5-5$

$m \sim n=0$

三.u

$\stackrel{2}{\sim} \approx \tilde{\sim} \sim$

ñ

$\therefore=\Omega n$

…ำ

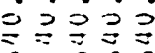

的访的

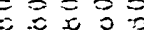

जि 15

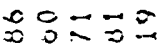

$\dot{x} \dot{j} \dot{j} \dot{0}$

$\approx \sim \bar{n} \vec{n}$

กิอยที

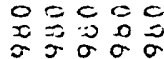

onings

- .

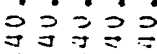

$\leadsto \sim n$

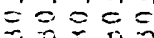

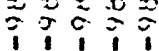

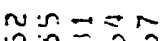

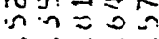

- $0 \dot{0} 0$

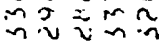

cै

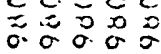

든.5

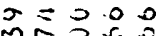

ज袺曹

舟完递

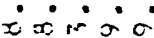

$-x x=$

으ㄴㅡㅗ 순물

$M M N N$

ज证品

กแก ก

$\Omega-0 \sim \cong$

$\dot{\overrightarrow{0}} \dot{\sim} \dot{\sim} \dot{\sim}$

in

ㅇํㅇㅊㅜ

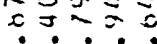

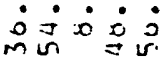

N N $\sim$ in

$\sim m \sim \sim n$

$\sim=n \sim n$

$\because 111$

con

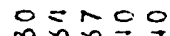

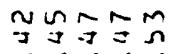

$m 0 \pi \alpha n$

nosio

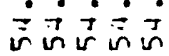

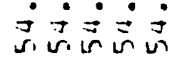

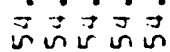

ำ

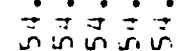

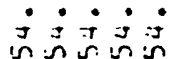

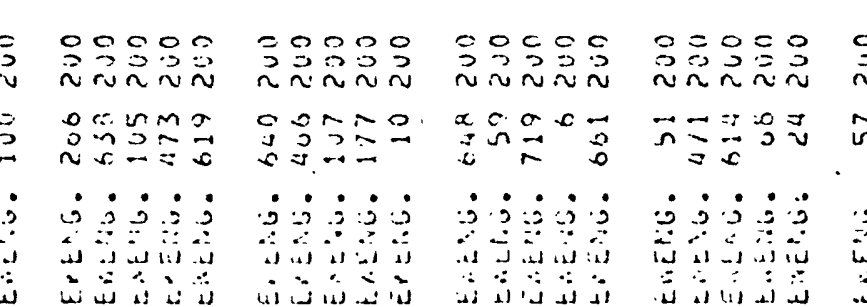

co:00

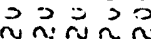

$n=20$

元仝宫

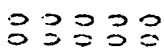
0ं0:0
옹영영 ○ंष्ठ
3300 0.3 $\therefore \therefore \circ$
20

ヒこังす

C $m a$ :

$\dot{0} \dot{0} \dot{j}$

in

cocino

$\sim \dot{0} \dot{0} \dot{0}$

a d

$\dot{j} \dot{2}=\dot{2}$

M

$\because 0=00$

$\therefore \div 0-1$

แอ 004

$\exists$ ?

$00 \div$

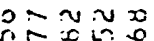

$\therefore$ í:

กิก

जे $\vec{i} \dot{\vec{t}}$

$=\pi \approx n$

$\operatorname{con} i s$

$\therefore \backsim \therefore \dot{0}$

onn

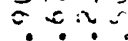

in $2=$

nNO $=0$

$\therefore \div 2 \div$

$\dot{0} \div \dot{0}$

$\leadsto \dot{m} \vec{n} \vec{n}$

c. 000

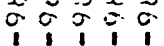

กฺ次

$\dot{i} \dot{i} \dot{\dot{1}} \dot{=}$

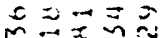

ที丶⿺𠃊八

$=0000$

1003

$\dot{0} \dot{0}$

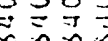

0

家分

No

$\dot{v} \dot{0}=$

enis

อิำ

$\sigma \approx 0 ;$

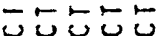

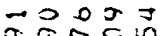

טu:

$\therefore \dot{0}=\dot{0}$

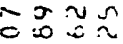

กำ

$\dot{0}=\dot{s}$

iv $n$ n.

$5 \mathrm{~m}=\mathrm{J}$ - $\cdot \dot{0}$ चั

ลำ

-

in

$\sim n-\sim \sim$

$\sim \sim \sim a$

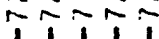

171

$\sigma \approx \approx$

$\overrightarrow{\vec{j}} \dot{\vec{r}} \dot{\vec{j}}$

m. $00 x x$ 
¿

8

- $O m n=5$

ํำ

$\dot{\sim} \dot{x} \dot{0} \dot{0}$

제 $0 \approx$

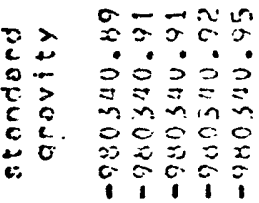

02
0
2
2
0
0
0
0

nNR =

$\div \div \div \div$

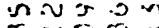

的访牙

oc.

* ここさこう

E minñ

รMำ

$\because \Xi \Xi \Xi$

ñvin

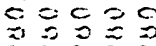

i 1910

$x+\leq 0=$

ง.?.า.

EDoni

oriunin

EDc. co

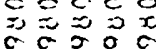

ラここここ

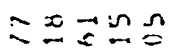

$\dot{0} \therefore \dot{\sim} \dot{\sim}$

0
5
0
0
3
0
0

$\approx n N m L$ $\because \therefore$ 至证司

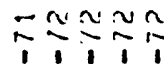

:

- vonimin

มาก

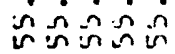

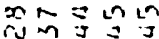

inisin

可可可 $\dot{\rho} \dot{-} \dot{m} \dot{\sim}$

ำ

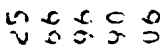

D퐁 $\therefore 0 \dot{0} \div$ $\because \cong \dddot{1}$

$\Rightarrow \overrightarrow{2}=0$

政

ำ 20

0000

$\because 30$

00000

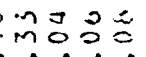
$\therefore 00^{\circ}$

onso ingiry. in

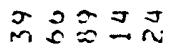
$m s \approx n$ i气

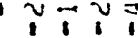

$x 2=0 \vec{x}$ $\because \because 3$. $\therefore \approx \equiv \dot{0}$

$\hat{=} \overrightarrow{0}=\ln \exists$ 0.0 $\pm \approx 0 \leq 1$

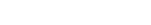

ㄷำ的气ミ

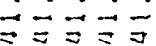
coco

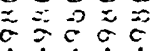

$0 \approx m n$ บ?

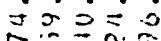
vinn

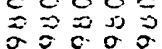

こここここ

$\hat{x} \vec{x}=$

c. 드.

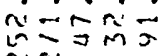

ñv

$-2005$ - 0. Djovin ต๓ะล ำำ

an nos. $=\operatorname{sกn~}$ 的的自 约比的年

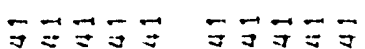

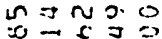
10 - ivis

옹ㄱ 0ं0ं0

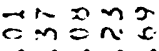
icं0。

$\stackrel{8}{=}=m: 0$ $\therefore \therefore \therefore$ "ñ

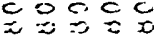
要过

mooon ?.? $\exists=n \leq$ $n \approx v 心$ 0000 $0 \%$

ここここテ n: $=0$ กุร.

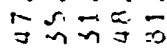
ñn

$50 \approx 50$

anmm

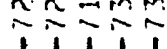

a $x=\simeq$ $\dot{n} \dot{n} \dot{n} \dot{n} \dot{n}$ すコすコす $\therefore \dot{0} \dot{0}$

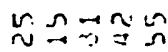

ำกำ वicंi

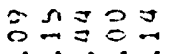
¿ं0ं0

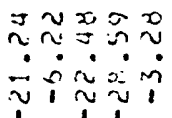

$2 x \approx 20$

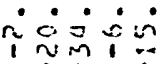

1

J舟西 $\therefore \dot{x} \dot{0} \dot{x}$

$\vec{a} \vec{x}=\tilde{n}$ $\therefore \dot{0}$

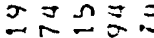
$\dot{0} \dot{0} \dot{0} \dot{0}$

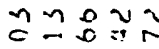
मीजिए पिजि s 0 en F

$m \approx \approx x N$ $\sim x \equiv \approx$

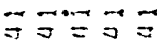
的里 ofcec

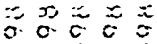
둥응

य $x \approx m$ $\therefore \dot{0} \dot{0}$ $\approx m: \pm m$ $\approx n v v$ m

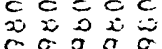

它こここ

in 0 ? ㄱ. 3.9

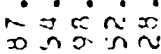

- aminis OND 0

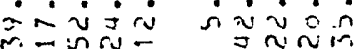

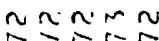

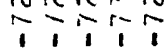

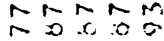
visini nivan $\underset{1}{1} \div \frac{1}{1}$

可可可

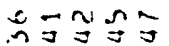
$\dot{v} \dot{v} \dot{v}$ in

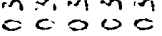
199.1

c $5=$ in $\dot{x} \dot{\bar{\Sigma}} \dot{0}$ $m \varepsilon=-$ ชบที

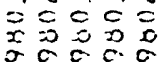

ヒこっこっ 으르

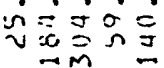

min movo in

0. กับ $\because \simeq \simeq ?$ บ

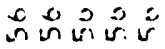

$r=0 m s$ $=\cap \Omega n$ $v \dot{v} v \dot{v}$

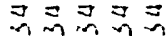
0 3005 1,111

可可要 $\dot{v} \dot{\sim} \dot{\sim} \dot{0}$ vin v cᄃc 0 - 0

ここすこう

a 403

$x x=$

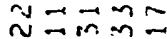

अ

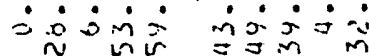
यกm- $\sim \sim n m \sim$ $1119 \div 111 \%$

ज宁可

$n \approx \infty<-$

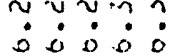

Nammin

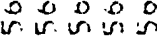
किं 0 $\because \because \bar{\square}$

$\vec{J} \widetilde{\exists} \vec{\jmath}$ aces=

에
OCE $0=0000$

c

- cmun

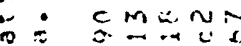

is

2 i.

$\left.\begin{array}{c}-\pi j \\ 0 \\ 0\end{array}\right]$

...

$\because 3: \therefore$ c. $250 \%$

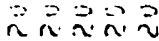

a. $\pi, x$. 0

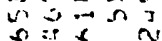
$\cdot \cdot \cdot \cdot$ $\therefore \therefore \therefore \therefore$ is is
옥도은 innar

a. van is $\therefore \tilde{n}=0$ $\therefore \therefore \therefore ;$
$00 \div 0=$

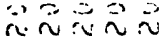

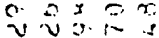
is

$\cdot \cdot \cdot$ osoce 踏贾

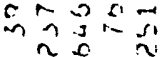
$\therefore \therefore \therefore \dot{3}$
OEO=0

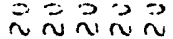
ñan c: $C$ C $c \cdot c$ ar ru n 


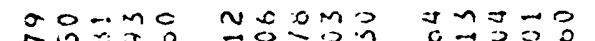

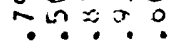

$\hat{1} \div 10$

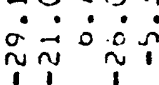

$x m \sim \approx$

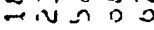

iिi的

กิํำ

van 0 in

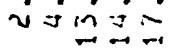

30003

$0.50=0$

$2933 ?$

$\therefore 0 \dot{0} 0$

$\therefore \circ \dot{0} 0 \dot{0}$

30303

$\therefore \dot{i} \dot{\sim} j$

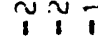

วิกนำก

$\dot{\mathrm{i}} \dot{\mathrm{i}} \dot{\mathrm{j}}$

$\therefore \therefore \dot{0} 0$

33339

$\therefore \dot{0} \dot{0} 0$

긍믕ㅇㅇㅇ

$\therefore \dot{0} \dot{0} \dot{0}$

뭉영

$\therefore \dot{0} 0 \dot{0}$

03333

$\because 3=3.3$

$\therefore \dot{0} 0 \dot{0}$

$5=3$

$\therefore \circ 0^{\circ}$

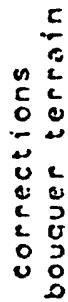

는요

n- mon $n=3$

í000 00000

经范气

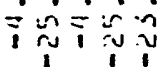

กำำ

$\therefore \dot{0} \dot{0} \approx$

$\because v \infty \ddot{\sim}$

$\because \div \div \div$

Nen $x$

$\cong 000$

$\because \because \because \because$

๓

ํำ的

$\sim \sim x \geqslant c$

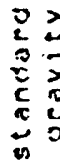

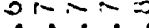

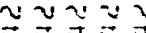

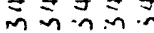

的济的

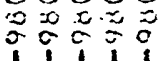

$\begin{array}{ll}1 & 1 \\ 1 & 1\end{array}$

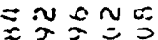
$\dot{v} \dot{i} \dot{n}$

$m m \propto \alpha$ in $m m \propto n \exists$

$\Rightarrow \quad m m^{m} \tilde{v}$

$\sum \approx \therefore \dot{0} \dot{0} \dot{0}$

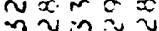

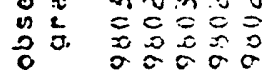

$0 \approx n 0-v$

$=0 x=0$

ขที่

$C_{2}=000$

व

* 55505

5055

$=\hat{\sim} \vec{\sim} \tilde{\sim}$

$-x>0=$

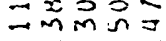

$0 \underset{n}{0} \approx \ddot{n} \stackrel{0}{0}$

EN

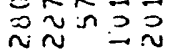

\begin{tabular}{l}
$c$ \\
$c$ \\
\hdashline 2 \\
0 \\
0 \\
0
\end{tabular}

$\sin n \in$

$0 m 502$

J $\Omega \Omega$

m $\sim_{M_{1}}=0$

$\dot{0} \sim \dot{\sim} \dot{\sim} \sim \dot{\sim}$

$\cong \simeq \simeq N$

$m m n=N$

2

w

iे

$\rightarrow \underline{x}$

$\ddot{x}=$

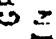

3

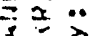

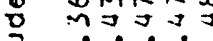

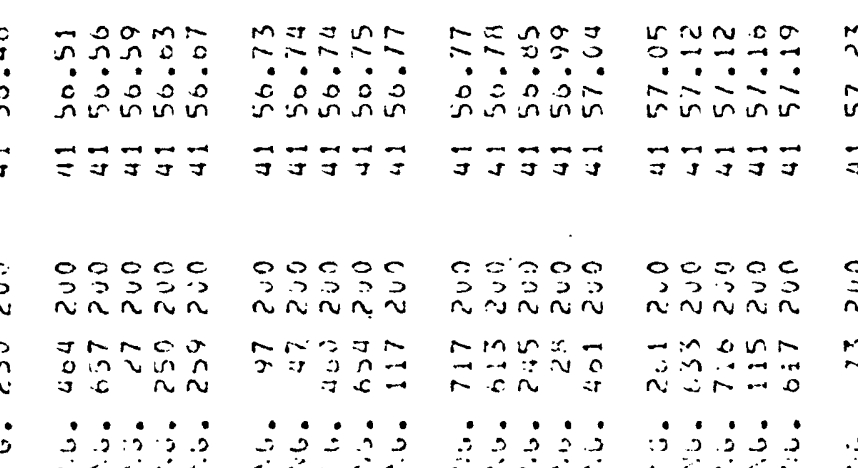

$\dot{0} \dot{0} \dot{0} \dot{0}$

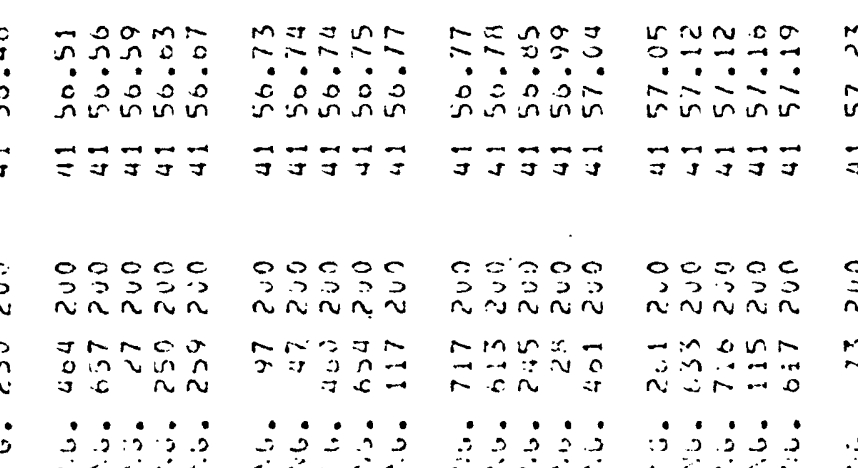

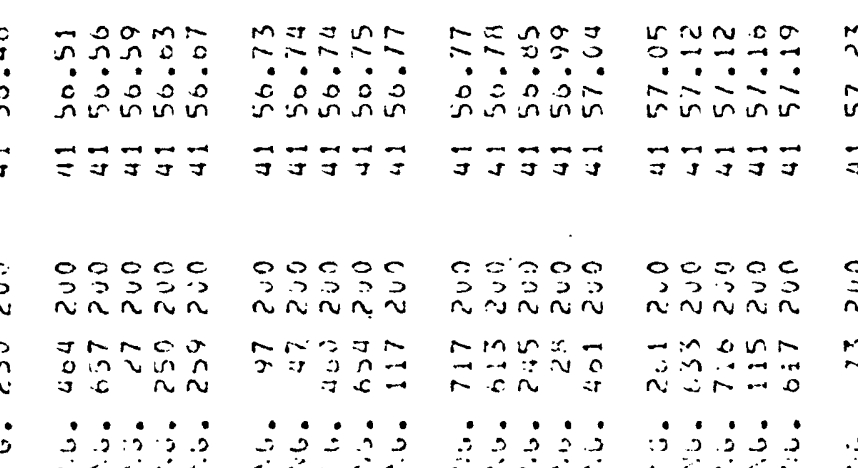

- $\vec{\exists} \vec{\Xi} \vec{\Xi}$

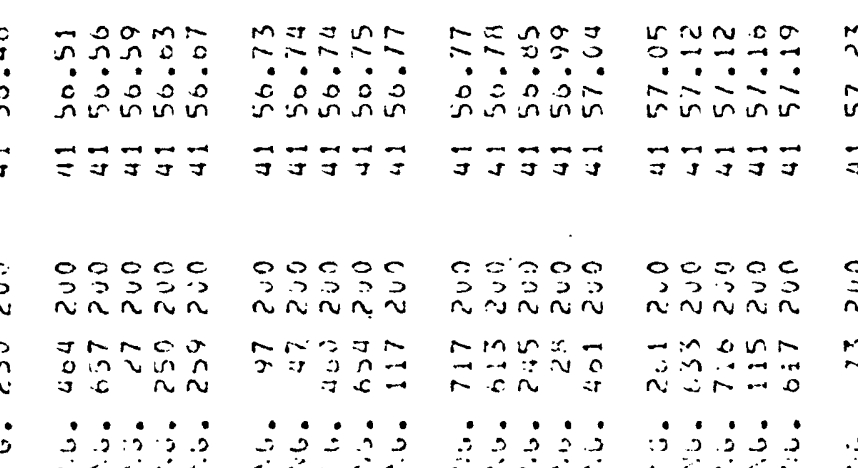

그는

¿ं: 0 :

กิก

$\approx J=00$

ro

$\dot{0} \dot{0} \dot{0}=$

$\therefore \therefore 000$

$\vec{M} \vec{M} M \simeq$

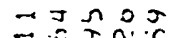

(2)

$\vec{\sim} \cong \pm 0 \pm$

c jisio

i $1 \frac{1}{1}$

$\equiv \dot{ \pm}=\dot{\square}$

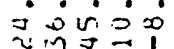

$\sim \rightarrow n \cap 0$

$\because 6 x=\frac{1}{2}$

$\therefore \simeq \dot{0}=0$

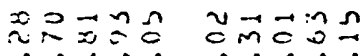

î $\overrightarrow{1}$

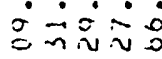

$\dot{c} \approx \dot{m} \dot{\sim} \dot{\sim}$

$152=m$

$\dot{\sim} \dot{\sim} \dot{n} \dot{n}$

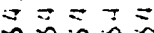

nก

$x=2 \vdots 0$

釷方?

$\sim 0-n=$

$n \cdots \cdots$

$\dot{0} \dot{x} \dot{x}$

$v \sim \tilde{v} v$

o $00=0$

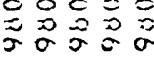

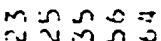

$\dot{n} \dot{n} \dot{n} \dot{n}$

$\vec{B} \ddot{\sim} \vec{\sim} \vec{\sim}$

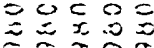

ज行

$m=\Sigma$ nn

$\because ? m n ?$

$\ddot{n} \tilde{c} \dot{\sim}$

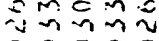

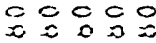

至 $\approx 00 \%$

ㄴํㄴㄴㅡ

可它5

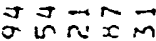

$\therefore \dot{0} \dot{\sim} \dot{0}$

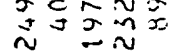

$x \sim 2 \cos$

$\because-0$ 约

$\because \cong \dot{0}=\dot{0}$

$m \equiv 心 \sigma$

5.0000 $\dot{n} \dot{n} \dot{n} \dot{\sim}$

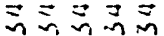

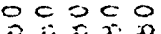

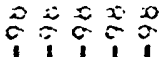

등

$\therefore \nabla>\rightarrow x$

$\therefore=2=$

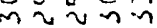

$c=000$

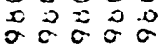

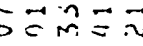

$\ddot{\sim} \vec{m} \equiv \dot{\sim}$ $\therefore=0 \therefore \circ$

$\therefore \therefore \dot{0} 0$

ำ응

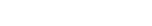

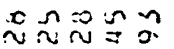

$=\hat{i} \sim \tilde{v}$

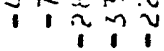

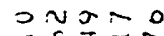

$\because 2=7$

ڤc

คกบนก

ำกำ

$\therefore \therefore \therefore \div \div$

$\therefore \sim \sim 0$

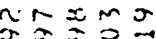

inisi:

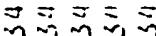

$\begin{array}{llll}c & 0 & 0 & 0 \\ x & 0 & 0 & 0 \\ \vdots & 0 & 0 & 0 \\ 1 & 1 & 1 & 1\end{array}$

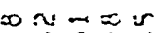

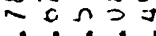

$\approx \cdots \cdots=$

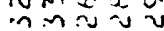

$00=00$

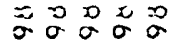

ப눈

$\vec{a}=\vec{C} \overrightarrow{0}$

눈둔

$\vec{x} \overrightarrow{0} \tilde{\sim}$

$\therefore \therefore \therefore \dot{0}$

可施尗

$\therefore \dot{0} \dot{0}$

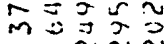

元分

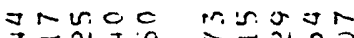

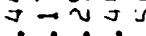

iññ

$\because \because ? \Omega ?$

$a \sin x=2$

in $0=2$

$\approx \sim m \sim \sim$

1: 111
象识

$\because \dot{0} \dot{0}$

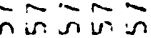

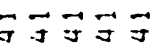

$=0=c$ น $\sim 2 n$

moms $c$

$s$
$\Rightarrow x 0=\pi$ ข $v \backsim m n$

$\dot{\Xi} \dot{\Xi} \dot{\Xi}$

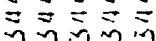

$\equiv \subseteq c 00$

i

nmmax

วงคว

$x$ 的戠

ง v

$=0003$

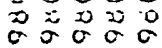

5ั05

non

$\dot{0} \dot{\mathrm{i}} \dot{\mathrm{i}}$

imir

$\sim \sim \sim \cdots N$

$\because \hat{1}$

$\because \Im \mathrm{g} \Omega$

$\therefore \therefore \therefore$

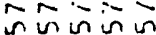

$\ddot{g} \ddot{g} \vec{j}$

의

$\dot{\vec{j}} \dot{\mathrm{J}}=$

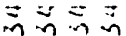

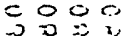

of

$c \sim-\cdots$

ก: : :

$x \vec{A} \overrightarrow{0}$

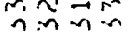

c:둥

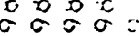

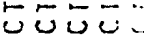

ษ $\approx$ in

imo:

in $\Omega 0$ in

$000 \mathrm{C}$.

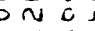

$\dot{m}-\dot{\vec{m}} \overrightarrow{\dot{m}}$

$\sim m N$

穴司兵

กภก

טnin

$\overrightarrow{7} \vec{x} \vec{j}$
2 $00-\pi$ 


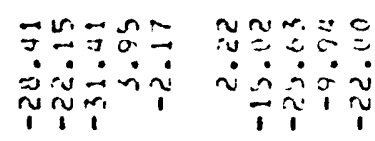

구요 0

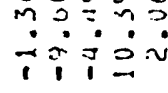

son $=0$

$\dot{0} \dot{0} \dot{\sim}$

1 im!

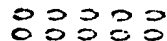

$\therefore \dot{0} \dot{0} 0$

웅영요응

$\dot{0} \dot{0} \dot{0}$

o

0
0
0

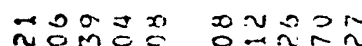

m.?

¿0ं0ृ:

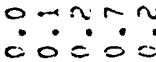

로요요

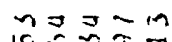

กับ?

s. $\because \cdots \div$

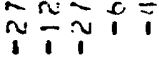

난

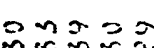

过昆立

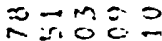

$\therefore \dot{0} \therefore \dot{0}$

$\approx$ ำ

$\exists=\varepsilon=2$

:

$3 ะ \div \div$

$\vec{B} \equiv \vec{B}$

c.

iा i i

$2 \pi \frac{203}{2}$

$\dot{\square} \dot{\square} \dot{0}$

$\sum \vec{\nabla} \equiv$

$==00$

i.

$O m=20$

충

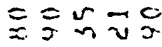

$\therefore \dot{0} \dot{0} \dot{0}$

Nimiñ

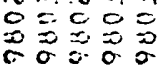

$n=x$

c

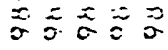

* ヒここここ

E $2 n \geq 200$

こここここ

구ㅇㅝㅛ

过立完立

西

in $\sin _{n}=\frac{a}{2}$

\begin{tabular}{ll}
$c$ \\
0 \\
\hdashline \\
0
\end{tabular}

กะเ์

$\exists r=0$

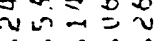
$\dot{\theta} \dot{i} \dot{i}$

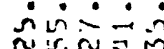

r. ariva

ำ?

nis ramo

$19 \div \div$

:

$\cos =0$

og.cat

$\because \div \div \approx$

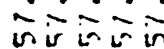

$\Rightarrow \because 303$

inis in in

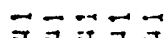

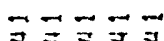

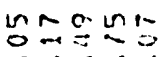

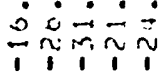

$=n n \sigma o n$

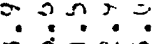

$1 \frac{1}{1}$

양ํㅇ

○ं0ं

ㄱำ

$\because \because \because \because$

-000:

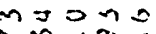

ำ?ำ.

$\ddot{\sim}=\bar{~}$

$\because \div \div \%$

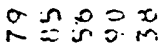

형ㅎํ

Nㅜㅁ

$\because \because \cdots ?$

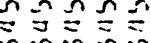

$\overline{0}=0$

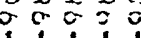

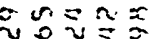

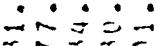

政江

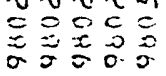

ヒேธธั

$v \rightarrow \infty=0$

$\because x=$

等出

cosin

$=02-5$

o

morna

$\div \div \div \div$

$\exists \equiv 0$ N

- ㄴ.

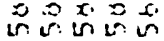

すすすすコす

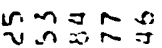

$\dot{i} \dot{i} \dot{i} \dot{0}$

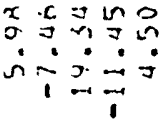

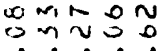

irisin

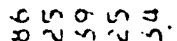

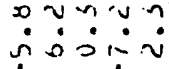

?

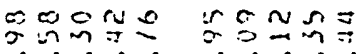

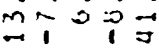

$2 m=51$

응공르영

$\therefore \dot{0} \dot{0}$

응로을

वंcं

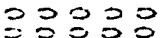

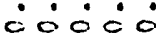

응영ㅇㅇ

óं宀

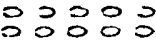

$\therefore \dot{0} \dot{0} 0$

กรกนข

acos

อง

$=m \sigma \sigma o=0 马 M \Xi$

$\therefore \leq 00: \therefore 00^{\circ}$

은 $น$

$=n \varepsilon \cdots=$

$\therefore \therefore c i 0$

$x \sin m=$

$n=0 \pi$

$\therefore \therefore \therefore \div:$

$x \rightarrow \hat{n} \sim \sim$

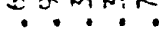

$\div \bar{i} \div \bar{q}$

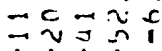

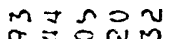

$m m n n z$

in $x x=$

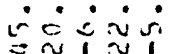

- $\cdot$.

iิ

きㅋㅀ

$\therefore \therefore=\therefore$

으를

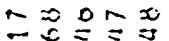

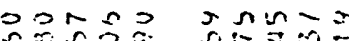

$\dot{\theta} \dot{\dot{m}} \dot{x}$

vivis $\dot{0} \dot{0}$

$\approx \pi 30$

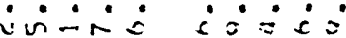

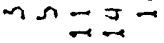

$\overrightarrow{5} \equiv 5=0$

$\operatorname{nan} x$

$\int \cos \pi x$

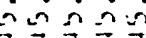

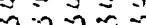

$c=0$

o $\vec{c}=\vec{c}$

sरोis

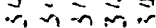

c. $0=0$

$x=5 \equiv 3$

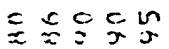

$\therefore \therefore \therefore \therefore$

芒菏

$==c=0$

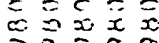

$=059 \%$

न

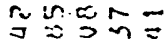

$\dot{0} \dot{2} \dot{0}$

CN N

$\begin{array}{llll}0 & c & c & 0 \\ 0 & 0 & 0 & 0\end{array}$

$\therefore \dot{0} \dot{0}$

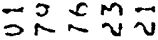

$m=0 \cong m$

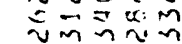

$\equiv \equiv \equiv \equiv=$

c c c

บ550

ปับั5

넌도

$0 x_{1}-x 0$

$\approx N \pi r=$

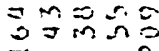

$\vec{D}=2 \pi 5$

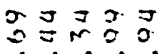

$\therefore \dot{x} \dot{0}=$

$0=230$

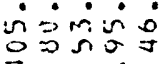

कóñ

- $\cdot \dot{0}$

जिंगे जि

$\dot{\vec{z}} \dot{\vec{i}} \dot{0} \dot{0}$

$D x==m$

$0: 0=\sum n$

$\Rightarrow \dot{\Delta} \dot{\sim} \dot{m}$

vruña

innm

mavmn

$\div \div \div \div$

$\div \div \div 11$

$n=n n N$

v'vmm

$m m m m n$

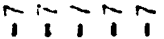

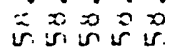

$\dot{x} \ddot{0} \dot{0} \dot{x}$

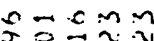

ระบนท

$\because 2050$

nnin n

की

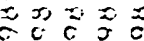

1111

-00?

ㄴ.?

$m \bar{c} \mathrm{c}$

mกn

c $0 \% \equiv$

$\begin{array}{lll}0 & 0 & 0\end{array}$

$5-\overline{0}$

숭

$m x$

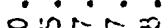

$\rightarrow \exists n \rightarrow \cdots$

a rin

구요의

$m-\min$

nNoum

$111 \%$

รูกะ๊ำ

$\because 0 \dot{0}=$

的的事

$c=5=$

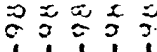

cc un

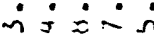

v. $\vec{\sim} m \sim$

$=0 c=$

o 000

$\bar{\omega}$

$m \geq n \geq J$

บำ

m的向:

r.

의의

向完它主

Ninn 2 in

11 11 $\dot{\nabla} \dot{0} \dot{0} \dot{0}$

oñ $x \cong x$ $\Rightarrow$ a

ar: o r

$\ddot{g} \vec{\Xi} \vec{J}$ 


\section{$0700 \%$}

$0 \nexists ⿱ 乛 ⿻ 上 丨$

ำกำ

अ $\therefore \dot{0}$

$\ddot{\sim}-\dot{0} \dot{\vec{n}}$

7

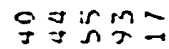

نำ

CMN 0

: $\because \div 9$

$\underset{1}{\dot{0}} \dot{\sim} \dot{\sim}$

83830

¿000.

33983

¿ல0்0

$n n x=n$

$\because \because \div 0$

0000 -

¿c

0
0
0
0
0
0
0
0
0
0
0

$\div$

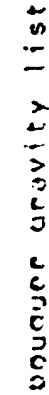

$\exists \because n-2$ 过守至

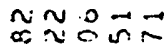

定穴的通

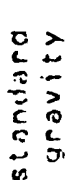

งิำำำ

$\therefore 0050$

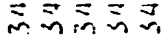

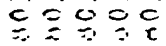

$5 \%$ i i

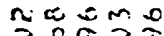

2
02
$2 \vdots 2$
0
0
0
0
0

?

200

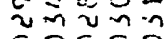

coc 0

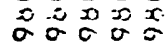

ต

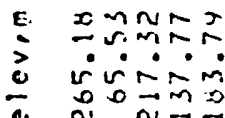

c

เง $\exists n n$

$\dot{i} \dot{j} \dot{i}$

nunam

กิT⿻

20

$20 \equiv I=$ $\dot{0} \dot{0} \dot{0}$

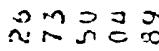
$\bar{i}=0 \frac{x}{1}$

$g x x \hat{n}=$ $\dot{0} \dot{0} \dot{0}$ ज位出

กำกักำ $\dot{0} \dot{0} \dot{0} \dot{0}$

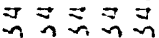
드응

i i i i

$\sim 00 \ln \pi$ نंنें

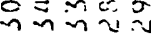

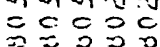
वूँ

은?

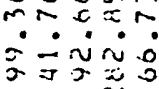

m n. no तिंडें -nnN-

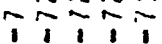

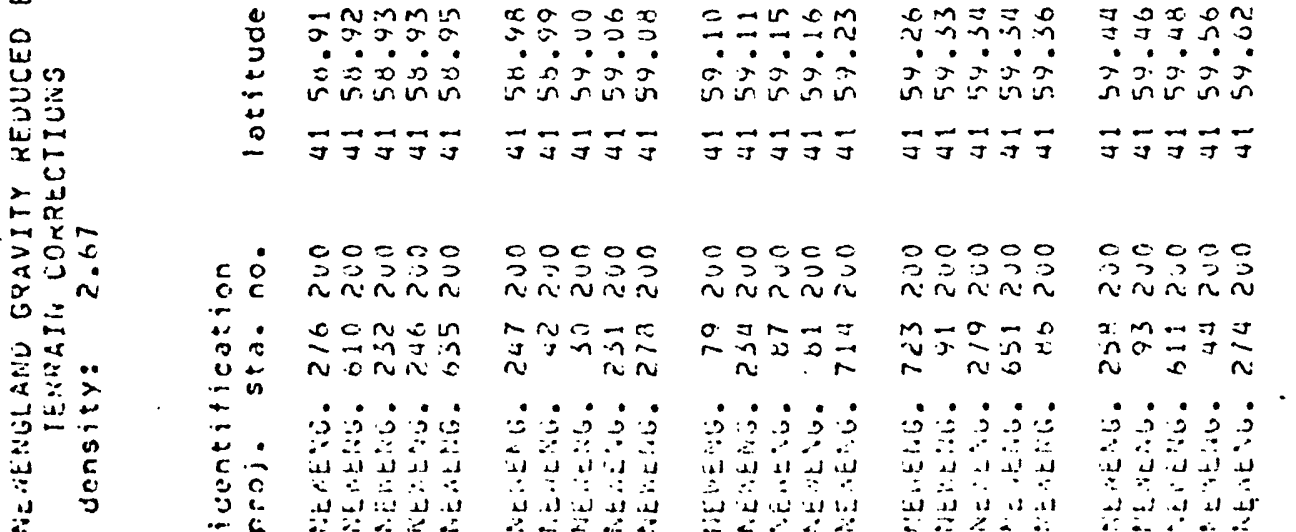

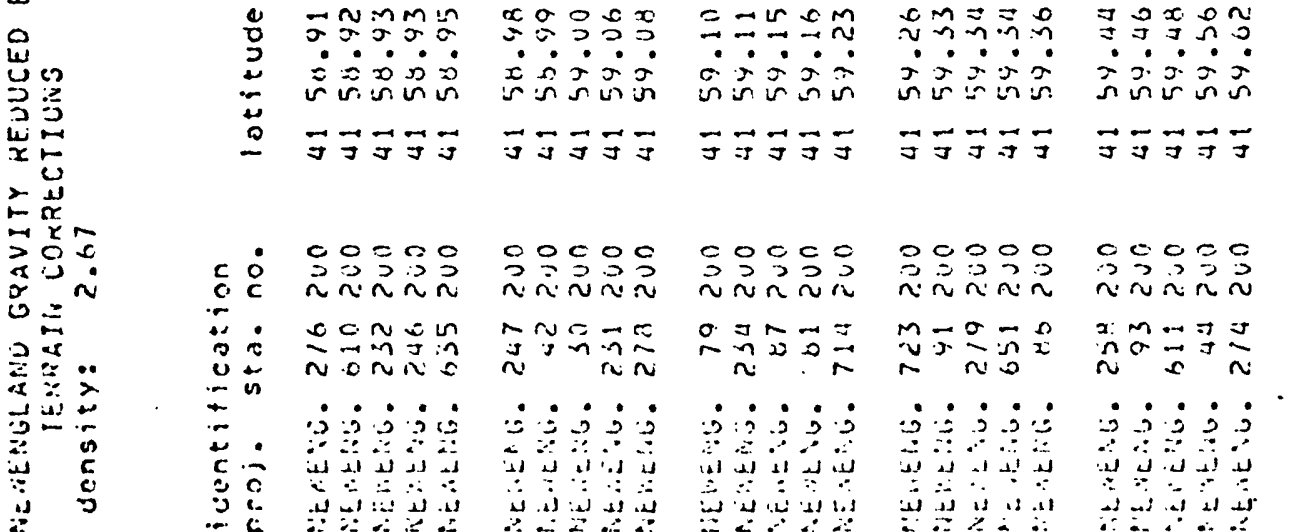

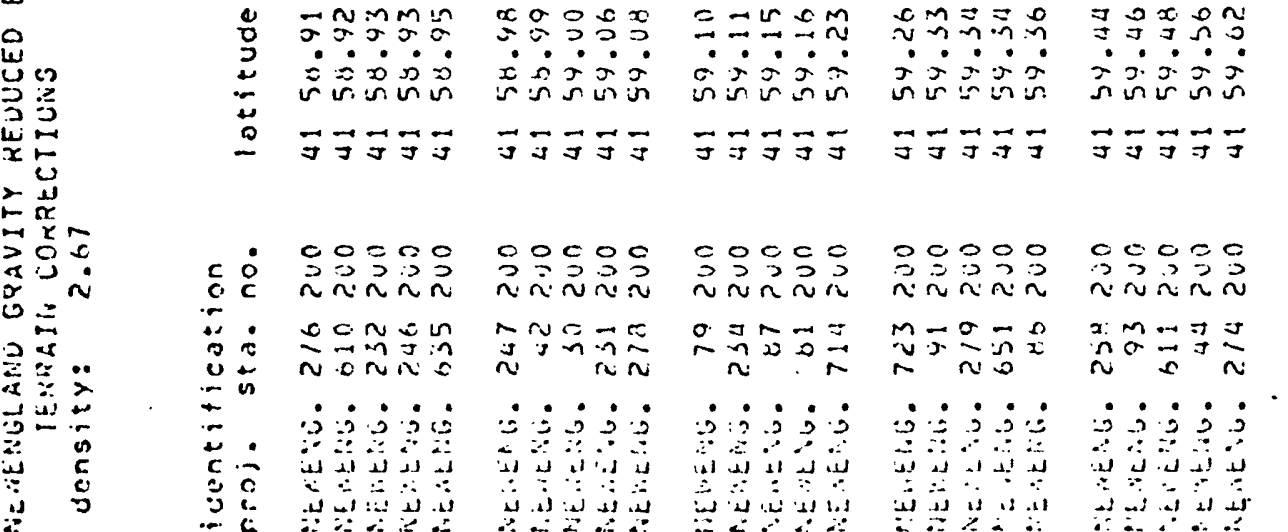

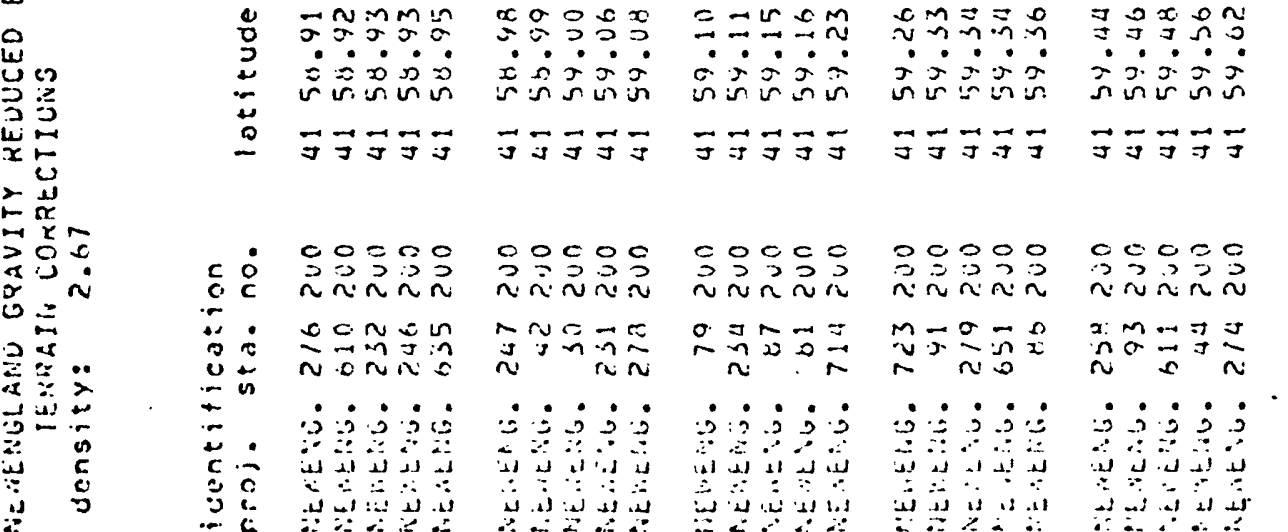

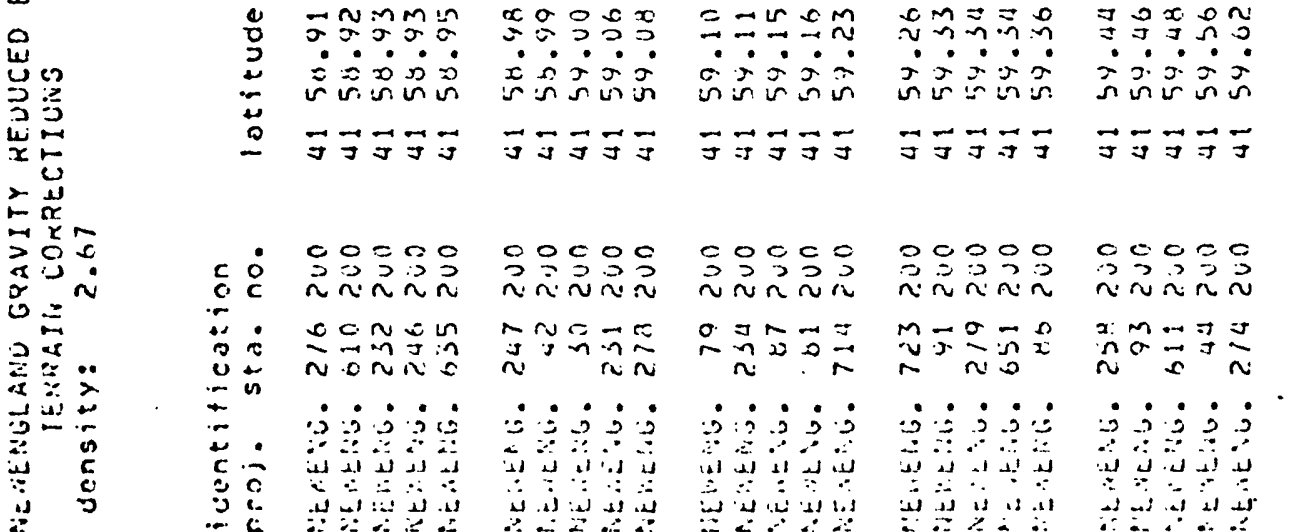

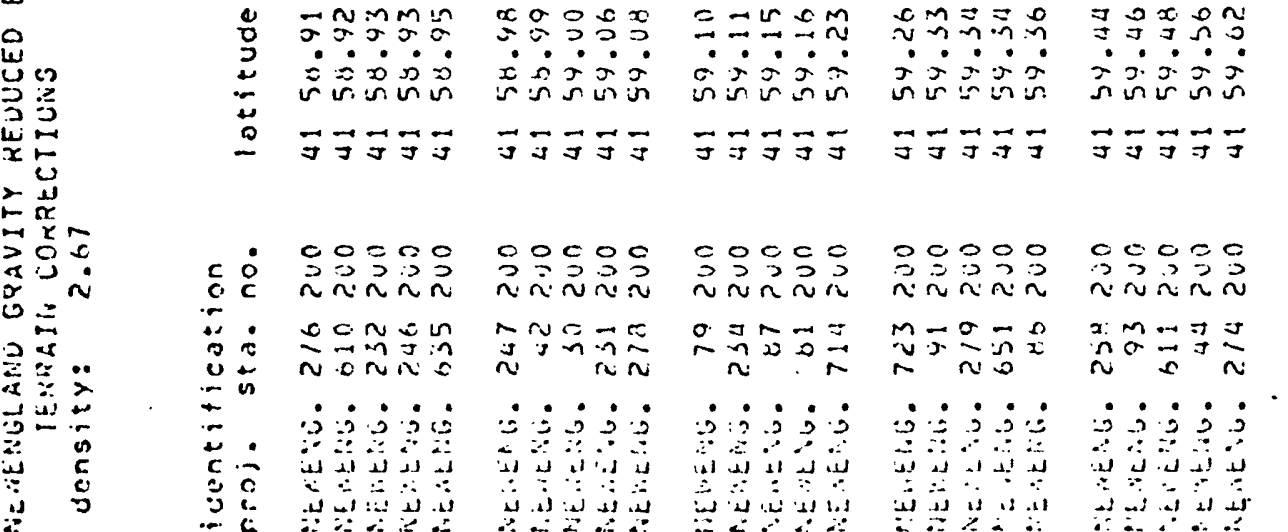

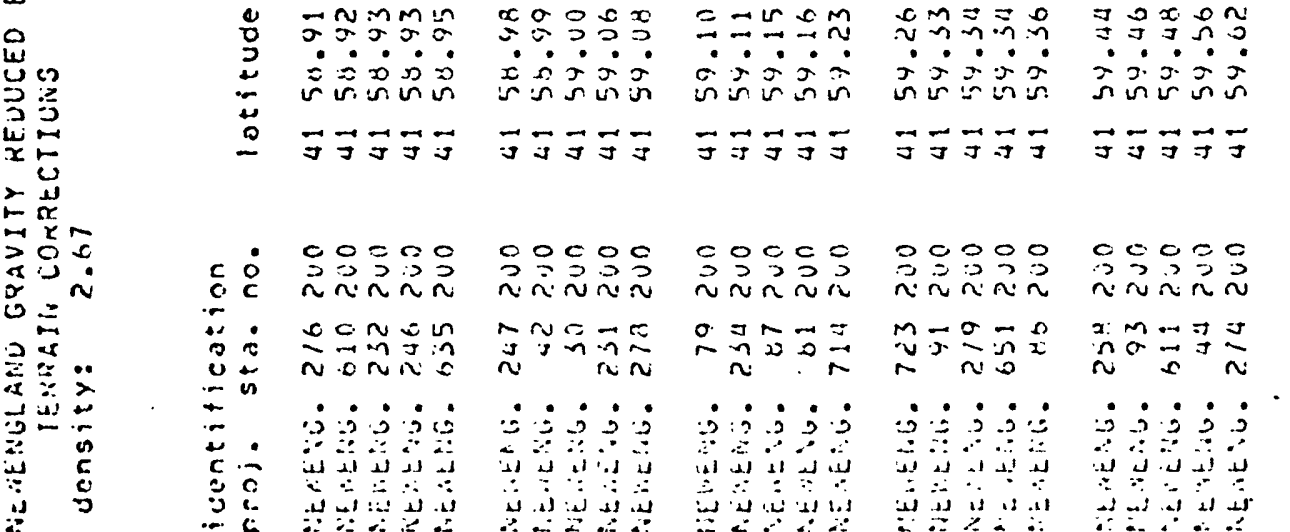

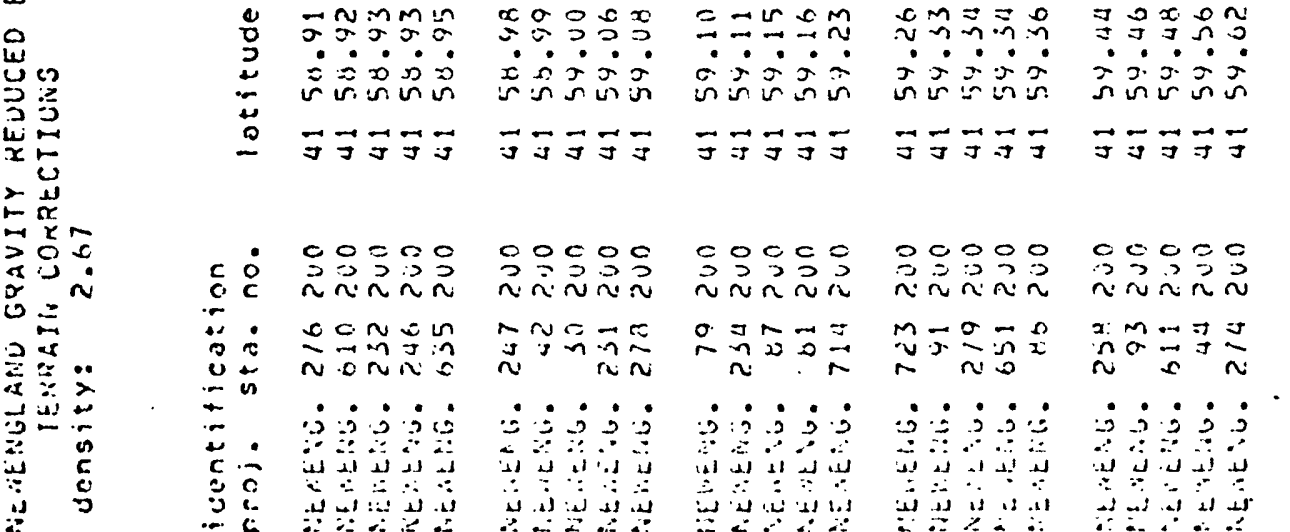

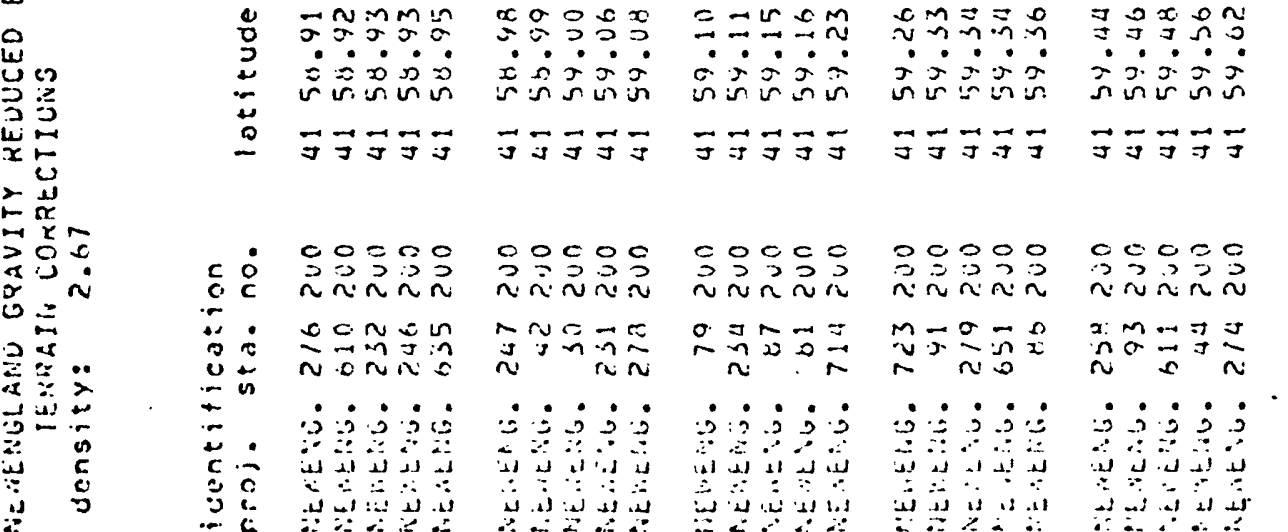

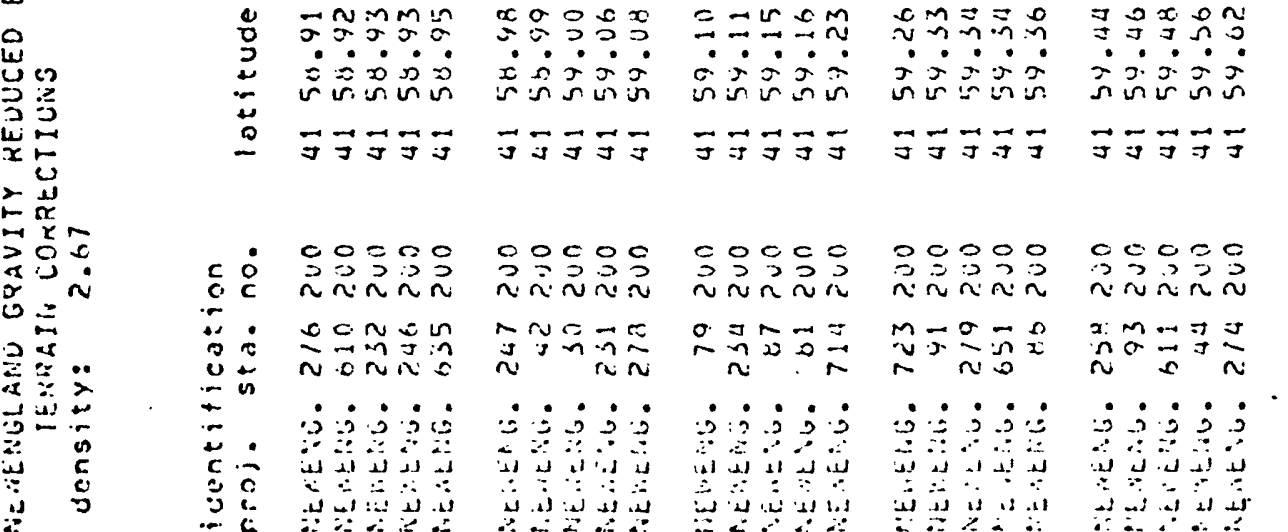

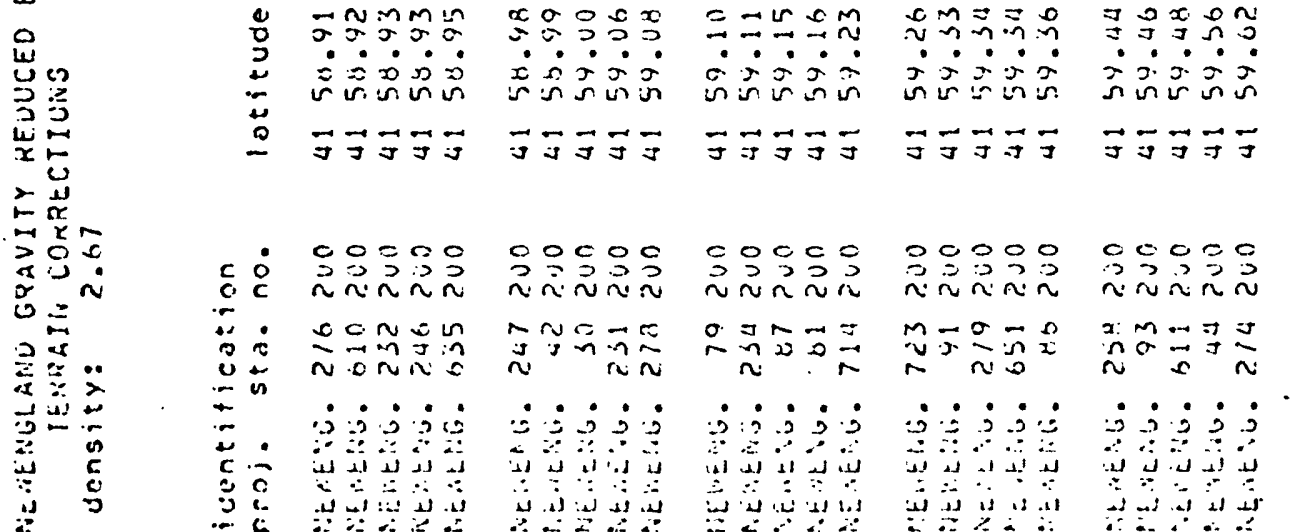

\section{ติ} i0ं0.

ใิำำำ வ00-

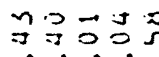
ऽं०்

$\therefore 5 \div=\frac{0}{0}$ نiำ

วท人天气 $\because \because \because \div$

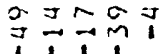
$\bar{m} x \approx m$

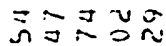

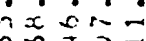
$\cong$ บำ

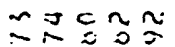
$\dot{0} \dot{0} \dot{0} \dot{0}$

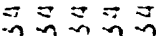

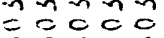
$=0$

옹ำ

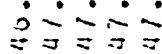
语的证 oc 000 वेश

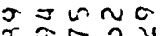
$\dot{i} \dot{i} \dot{0}$

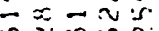
$n=\tilde{n} n$ $\approx 0000$

1,11

의 $\dot{0} \dot{n} \dot{0} \dot{0}$

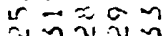
$c=0=0$

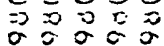

$\vec{m}=\sqrt{n}=$

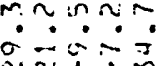
18

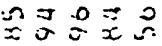

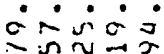

$\exists \stackrel{\sim}{*} \stackrel{n}{=}$ $\therefore=000$

$\because ت O Q=$ -000:

220 ¿ंc

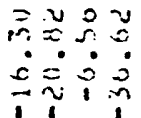

$\vec{x}=0 ?$

iั $\overline{1}=\bar{i}$

Nกn

$\because m=$

กำ

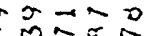

ขึกฐ

$\stackrel{0}{0}=$

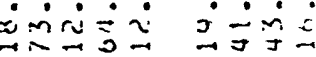

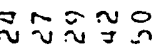

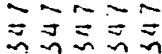
$\circ 00=0$

今० 0

에 $x=\hat{0}$ in $-5=0$ 列主 ทททท丶丶 年 क $5=5$

들

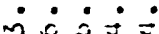
o. vำ 0
$x=0$
0

ñun $\because 20.2$

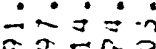

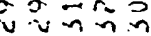

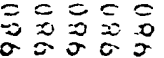

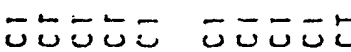

๒ேேேั

ธே兀゙

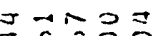

not 02

$\because \approx 000$

x. $0=x, 5$

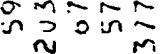

$\sim \vec{\sim} \sim \overrightarrow{0} 0$

$0 \approx \sim \vec{m}=$

$\because \sim \because \dot{m}=$

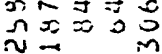

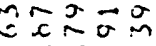
$\overrightarrow{0}=0 \sim n$

ํํㅇํ워 $\dot{\vec{n}} \dot{\dot{\sim}} \dot{\mathrm{m}}$

or $\approx$ in $m$

$\rightarrow \Omega v m \cdot$ $\because \hat{n} \stackrel{\dot{0}}{=} \dot{m}$

$m \sigma \approx n \approx$

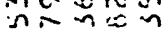
$\therefore \dot{\sim} \dot{\sim} \dot{0}$

กัำำ NNnNM $m \sim-n \simeq$ T1: 11

$\div \div \div \div 1$

$\simeq \approx \sim \approx N$

$\simeq \approx 2 \pi \frac{1}{1}$ $\therefore \dot{0} \dot{0} \dot{m}$ $\sim-\sim \sim n$ $\div \div \div \div$

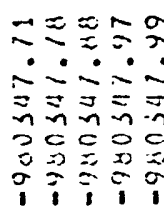

$\vec{F}=m m$

$\dot{0}=\dot{0} \dot{0}$

nับข

드을

$\Rightarrow 00$

ㄴํㄴㄴㄷ

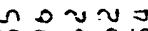

? $\therefore \dot{0}=0$

$\overrightarrow{r u} \sim_{n}=$

$x \backsim 50=$ $\therefore \dot{i} \dot{i} \dot{m}$ Nn次

$\sim m \cdot v \sim$

$\div \div \div \%$

$\approx$ aru? $\because \simeq v$ $\equiv \ddot{z} \approx$

$n:$

둥

둥

은은

$\therefore \dot{0}=$

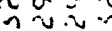

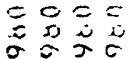

5

$303=$ in omn

$\rightarrow-$

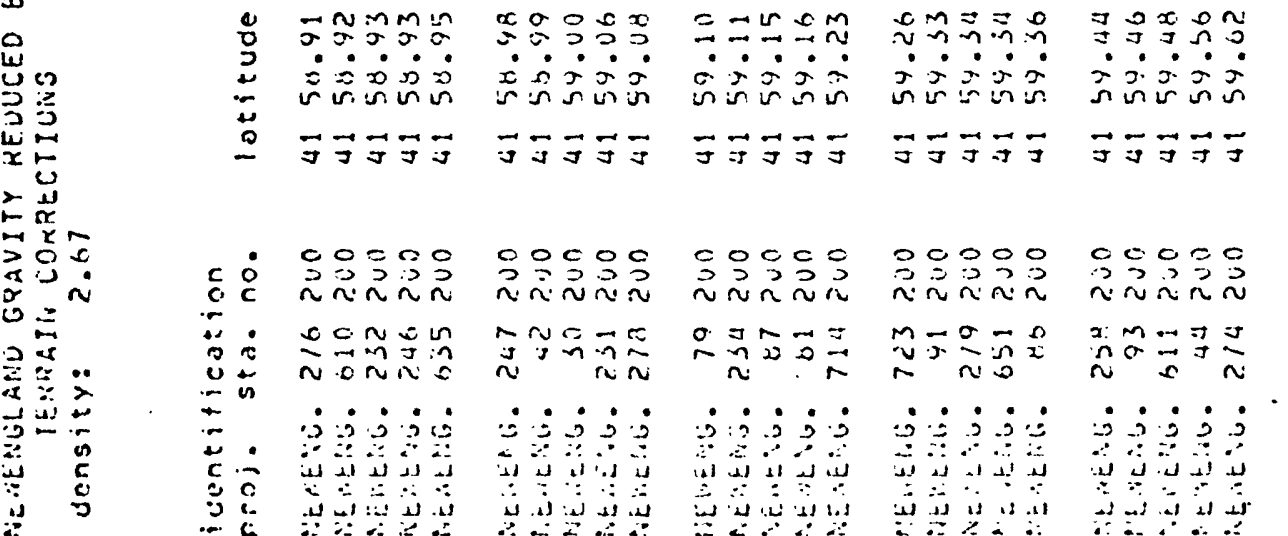

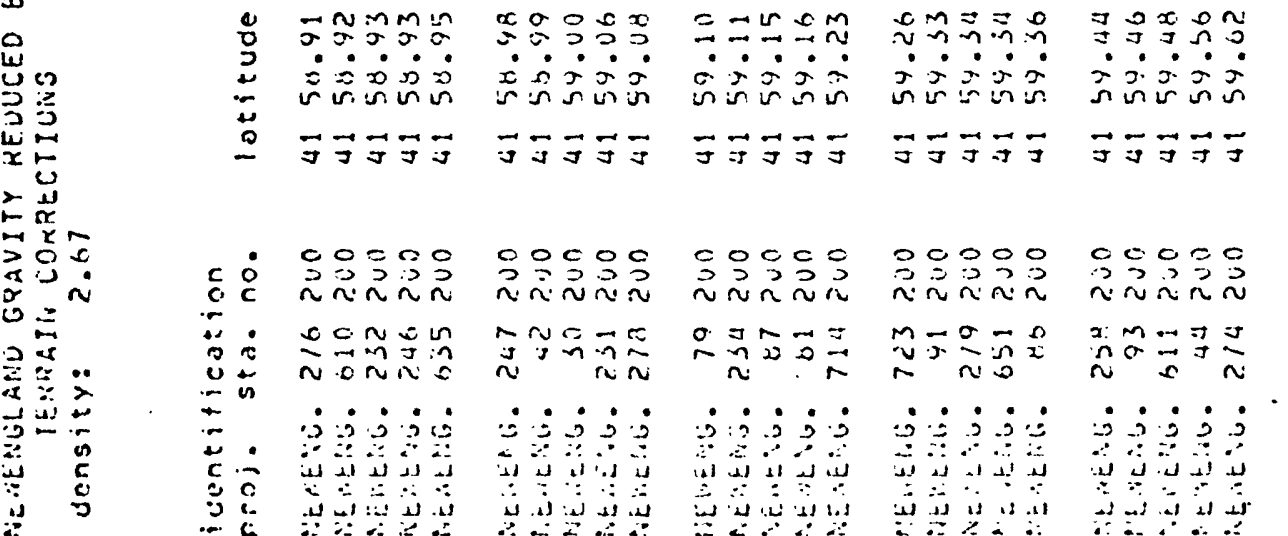

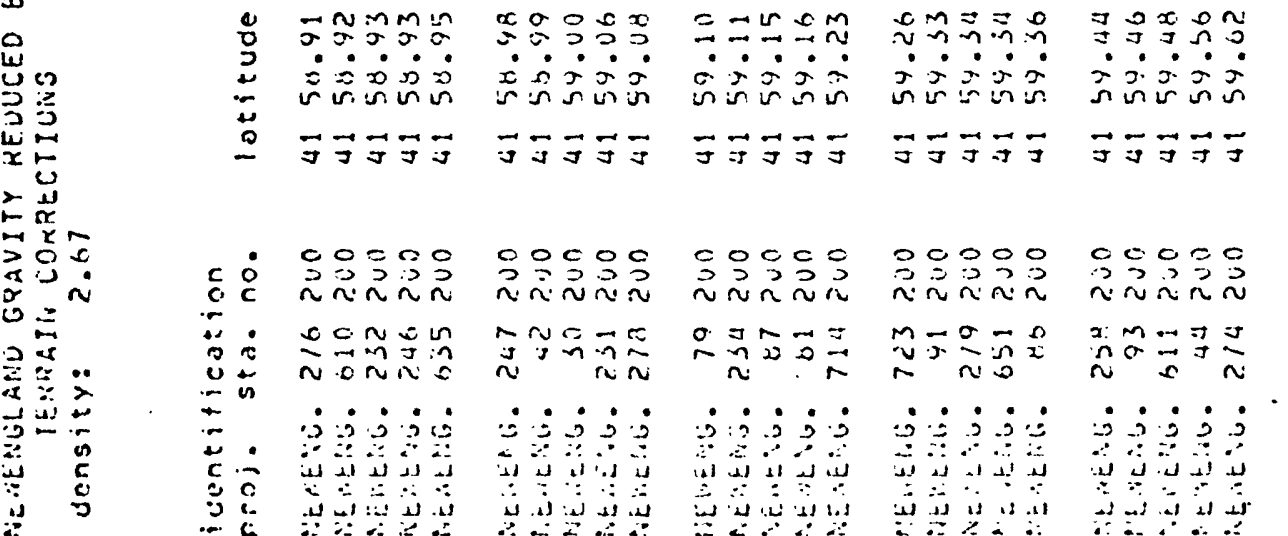

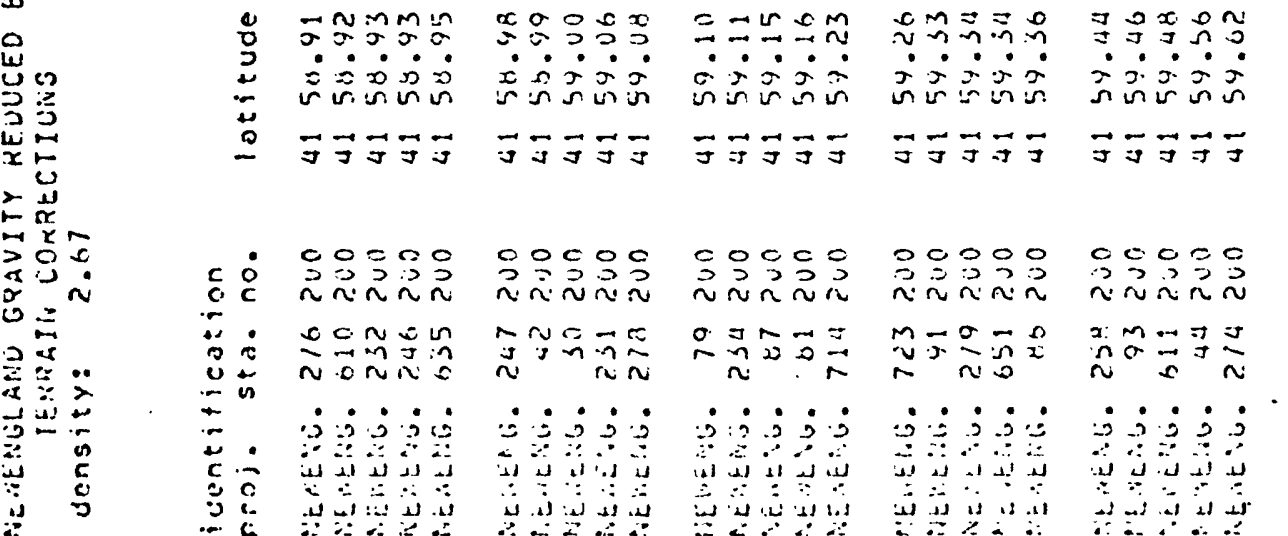

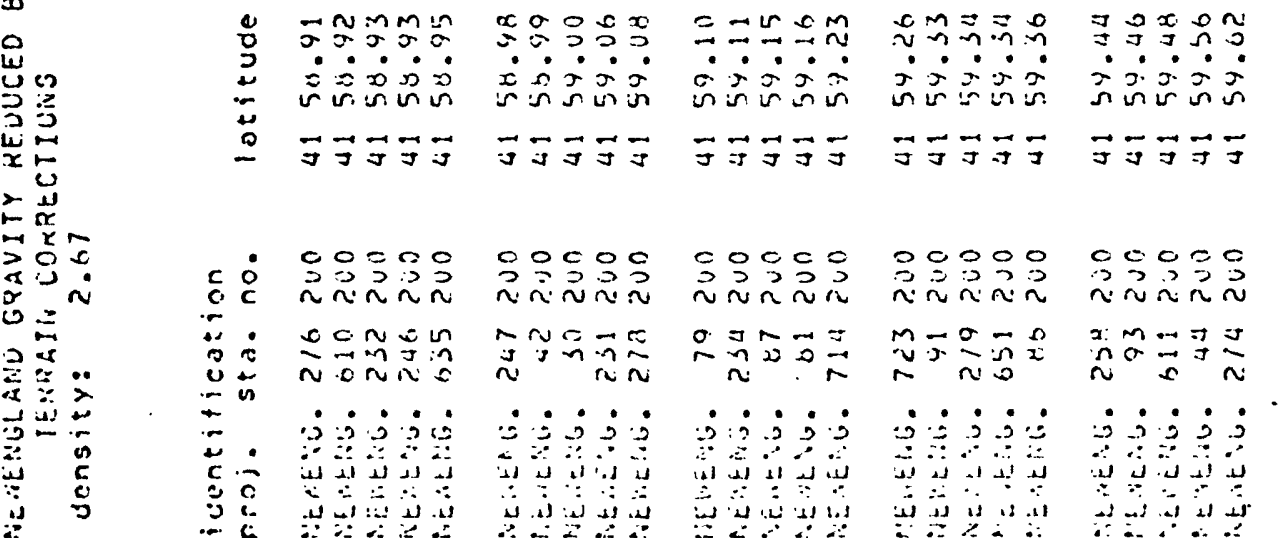

\begin{tabular}{|c|c|c|c|}
\hline 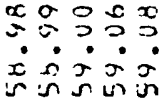 & 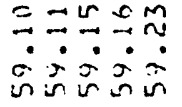 & 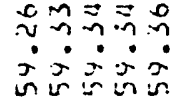 & 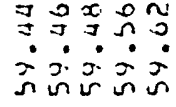 \\
\hline$\vec{j} \vec{j} \vec{j} \vec{s}$ & $\vec{\Xi} \vec{\exists} \vec{\Xi} \bar{J}$ & $\vec{\nabla} \vec{J} \vec{J} \vec{J}$ & $\vec{J} \vec{j} \vec{J} \vec{J}$ \\
\hline 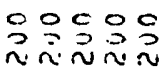 & 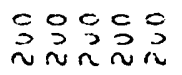 & 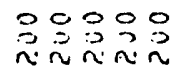 & 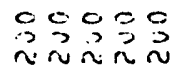 \\
\hline 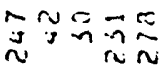 & 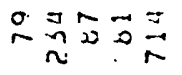 & 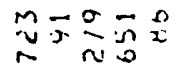 & 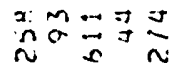 \\
\hline 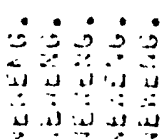 & 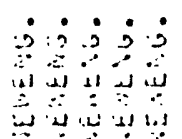 & 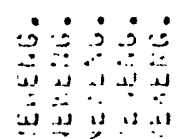 & $\therefore$ \\
\hline
\end{tabular}

ñm nin in in

$\approx 03 m=$

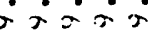
in in in in

缽学学

m웡의 - jE थ 4 in nam

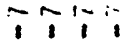

J00 $\dot{0} \dot{0}$ in

$\bar{a} \pi \tilde{\pi}$

00000 ữ

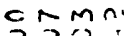

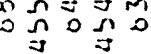
$0 \mathrm{v}=$

$\dot{0}: \dot{0}$ तñ ㄴ. 


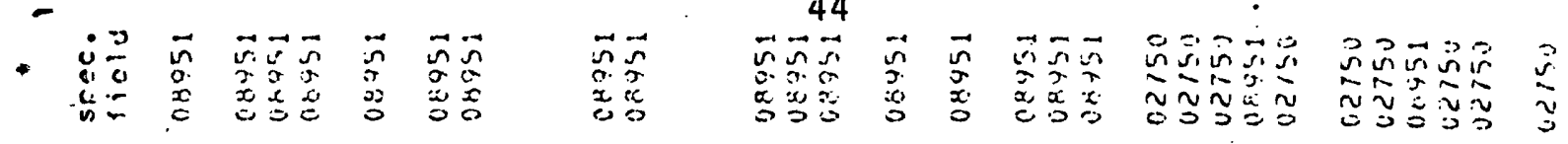

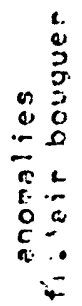

:
च च $\dot{\sim} \dot{0} \dot{0}$ तथ๊

is

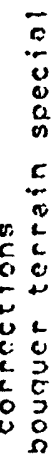

:

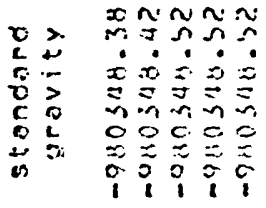

$\begin{array}{ll}= & 2 \\ 0 & 2 \\ 2 & = \\ 2 & 3 \\ 0 & 0 \\ 01 & 2 \\ 0 & 5 \\ 0 & 0\end{array}$

mก⿻․ $\therefore \dot{0} \dot{0} \dot{0}$ vñm

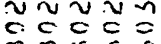

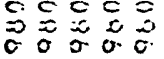

u

E 으욜

-

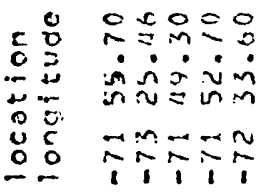

ํำํำ =ंத்

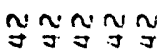

$\therefore 0=\sin$ injin $\because-\frac{1}{1}$

Jos inmív जin

90930

a mmo $\dot{x} \dot{i} \dot{\sim}$ चा

om $x$

$\therefore i$ i i

$\dot{0} \dot{0} \dot{0} \dot{0}$

30003

cं0்

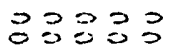

$\therefore \circ \dot{0}=$

영영 विं

93093 웅ㅇ

23030

$\dot{0}=0 \dot{0}$

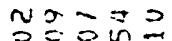

บัM

IN

0ं0 00

$\therefore \dot{100}$

$\therefore 000$

ํำ는

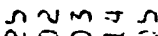

กรั íं $\dot{0}$

$\therefore \dot{0}: \dot{0}$

íं०:

I $\approx$ in $\vec{\exists}$ an $=$

$\vec{s} \dot{m}=2$

zan

$n n s=v$

$\approx i m \tilde{n}$

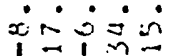

$\checkmark m \backsim \simeq \vec{i}$

ขบค

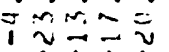

$\therefore \div \div \div$

워요

$\because \div \tilde{i}$

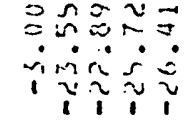

$\Rightarrow \approx \approx \approx 00$

$\therefore \hat{m} \vec{i} \approx$

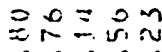

$\because$ iomi

$\sim \dot{i} \dot{\sim} \dot{0}$

$m=0 \Omega H$

$\because \div \div \div$

ñ⿻

ำตำ

ำ⼳.

$\ddot{i}=\dot{\sim} \underset{\sim}{\dot{v}}$

$\therefore \dot{0} \dot{0}$

$\approx \exists \hat{n}=0$

$\because \because \dot{c} \div=$

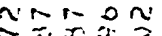

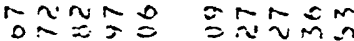

$\dot{x}=\dot{0} \dot{0} \dot{0}$

$\vec{F} \vec{\nabla}=$

nnm

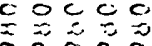

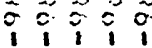

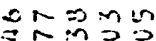

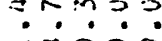

$n \equiv \equiv$

n约的

$\begin{array}{llll}0 & 0 & 0 & 0 \\ \sigma & 0 & 0 & 0\end{array}$

$\dot{0} \dot{0} \dot{0}$

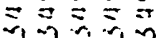

$00=00$

o

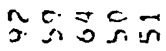

$\therefore \therefore \dot{0}$

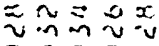

$0=0$
0

急

$\cong \tilde{v} \tilde{\sim} \tilde{0}$

NAn

$\therefore \approx \therefore \dot{2}$

$\because n=\hat{c}$

$\because$ 至站

o 111

$\dot{0} \dot{0} \dot{0} \dot{\Omega}$

วิ

c 0 C $=0$

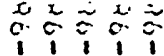

numag

$00.02 x$

$\ddot{i} \dot{0} \dot{0}$

ñ.

cำ

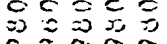

$3 \div=n-$

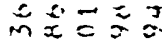

n的证

$\cong \equiv \equiv 0$

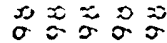

Nㅗㅇㅛ

arkn

$\dot{j} \dot{j} \dot{0}$

$\because n m n n$

$\begin{array}{lll}0 & 0 & 0 \\ x & 0 & 0\end{array}$

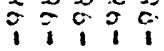

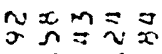

$\dot{\sim} \dot{i} \dot{j} \dot{j}$

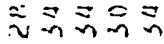

$\approx 0 c 0 c$

$2005=$

요물

nnm n

웡요

i

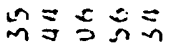

$\dot{n} \dot{0} \dot{0}$

$\overrightarrow{\dot{v}} \tilde{v} \bar{v}$

co $=0$

$\begin{array}{lll}0 & 0 & 0\end{array}$

$5 \quad 55$

30,3050

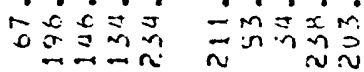

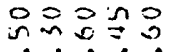

.5

min

ำㄹํㅇำ

is $5=\therefore$

30000

mon

misis

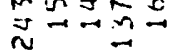

$r_{i}=\vec{x} \geq \vec{z}$

ni - irini

$\rightarrow-202$

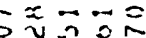

$\vec{v} \vec{r} \vec{\sim} \vec{s} \vec{s}$

nnmnn

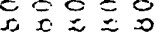

i $\bar{i}=\bar{i}$

$0 \rightarrow \pi n$

บ? ป?

$\rightarrow n=\Omega$

กv v v

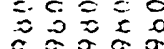

ธัธே

$\vec{n} \approx 0.50$

i-jim

rusin

c. $0=0$

cNo

$=0020$

$\therefore \dot{-1} \dot{0}$

둬원웅

$000=0$

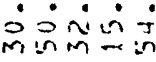

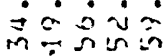

inis $\dot{x}$

$000 c 0$

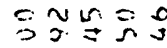

$\vec{m} \overrightarrow{0} \mathrm{x}$

$n M-m e n$

monmm

$2=\simeq M$

Nmu-

mmin

$\dot{0} \dot{2} \dot{0} \dot{0}$

$\ddot{-} \dot{i} \dot{i}$

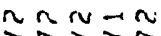

$\sim \approx-\sim \pi$

mann

11111

Ti: mnimo

证

c. 350

o 50

$=\vec{i} \vec{i}$

vง v $v$

$\therefore \therefore \therefore ?$

iil ii is
두웅

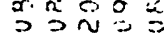

o.

$\vec{n} \overrightarrow{0}$

v v v v

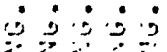

زئ
N०050

$\because n i n ̃$

$\vec{c}$ in 5 . $c$

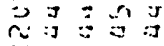

$\therefore \therefore \therefore$

$0 \%$

药 
总

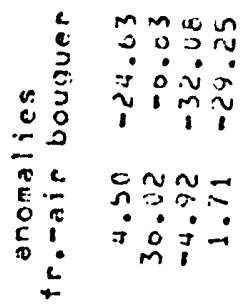

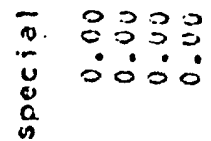

달

胥

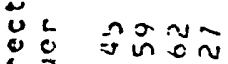

$2 \frac{1}{c}$

0

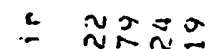

i $\div \div \div 0$

$\therefore \quad \omega$

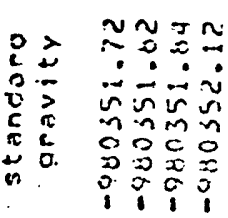

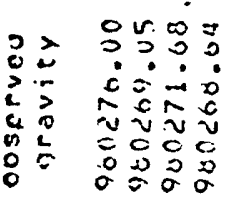

is $\bar{u}$

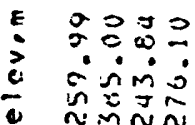

is

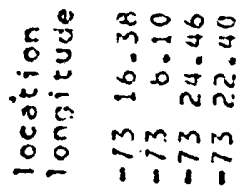

2े

بิ

:

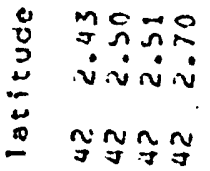

$2 \frac{1}{x}$

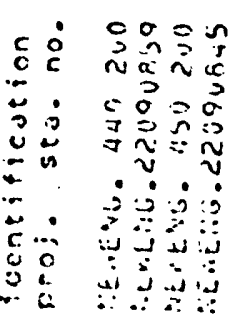

